
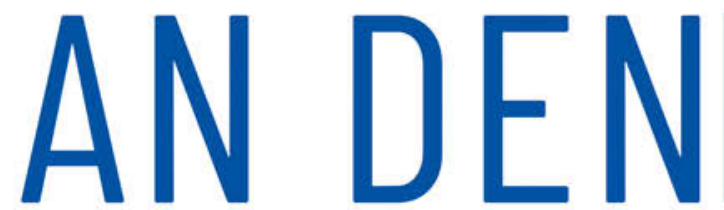

Maximilian Pichl, Timo Tohidipur (Hg.)
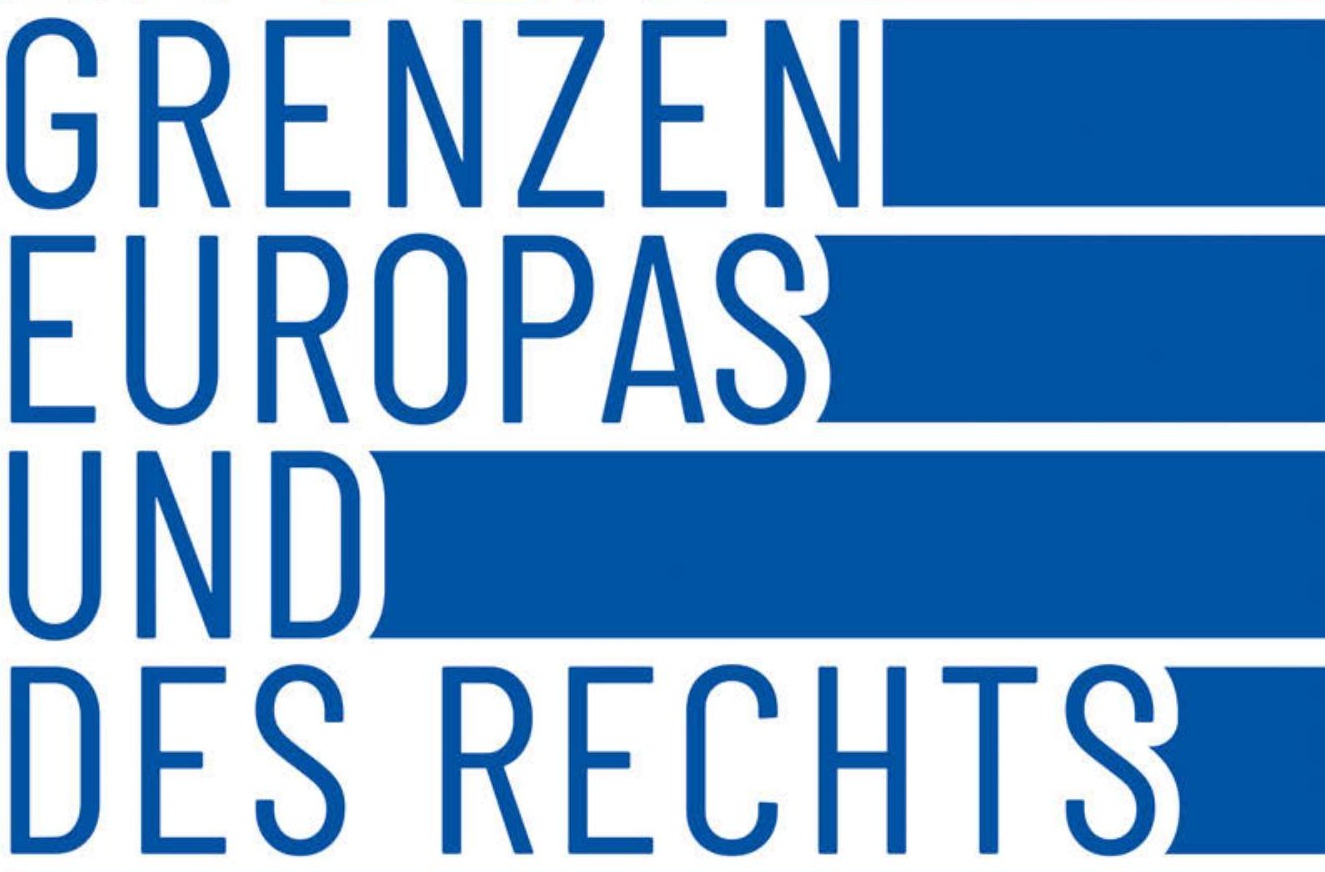

Interdisziplinäre Perspektiven auf Migration, Grenzen und Recht

[transcript] Edition Politik 
Maximilian Pichl, Timo Tohidipur (Hg.)

An den Grenzen Europas und des Rechts

Edition Politik | Band 74 
Die freie Verfügbarkeit der E-Book-Ausgabe dieser Publikation wurde ermöglicht durch den Fachinformationsdienst Politikwissenschaft POLLUX

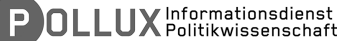

und ein Netzwerk wissenschaftlicher Bibliotheken zur Förderung von Open Access in den Sozial- und Geisteswissenschaften (transcript Open Library Politikwissenschaft 2019)

Bundesministerium der Verteidigung | Gottfried Wilhelm Leibniz Bibliothek -Niedersächsische Landesbibliothek | Harvard University | Kommunikations-, Informations-, Medienzentrum (KIM) der Universität Konstanz | Landesbibliothek Oldenburg | Max Planck Digital Library (MPDL) | Saarländische Universitäts- und Landesbibliothek | Sächsische Landesbibliothek Staats- und Universitätsbibliothek Dresden | Staats- und Universitätsbibliothek Bremen (POLLUX - Informationsdienst Politikwissenschaft) | Staats- und Universitätsbibliothek Carl von Ossietzky, Hamburg | Staatsbibliothek zu Berlin | Technische Informationsbibliothek Hannover | Thüringer Universitäts- und Landesbibliothek Jena (ThULB) | ULB Düsseldorf Universitäts- und Landesbibliothek Düsseldorf|Universitätsbibliothek Erfurt | Universitäts- und Landesbibliothek der Technischen Universität Darmstadt | Universitäts- und Landesbibliothek Münster|Universitäts- und Stadtbibliothek Köln| Universitätsbibliothek Bayreuth | Universitätsbibliothek Bielefeld | Universitätsbibliothek der Bauhaus-Universität Weimar|Universitätsbibliothek der FernUniversität Hagen|Universitätsbibliothek der Humboldt-Universität zu Berlin | Universitätsbibliothek der Justus-Liebig-Universität Gießen | Universitätsbibliothek der Ruhr-Universität Bochum | Universitätsbibliothek der Technischen Universität Braunschweig | Universitätsbibliothek der Universität Koblenz Landau| Universitätsbibliothek der Universität Potsdam | Universitätsbibliothek Duisburg-Essen | Universitätsbibliothek Erlangen-Nürnberg| Universitätsbibliothek Freiburg | Universitätsbibliothek Graz | Universitätsbibliothek J. C. Senckenberg an der Goethe-Universität Frankfurt | Universitätsbibliothek Kassel | Universitätsbibliothek Leipzig| Universitätsbibliothek der LMU München | Universitätsbibliothek Mainz|Universitätsbibliothek Marburg| Universitätsbibliothek Oldenburg| Universitätsbibliothek Osnabrück | Universitätsbibliothek Siegen | Universitätsbibliothek Vechta | Universitätsbibliothek Wien | Universitätsbibliothek Wuppertal | Zentral- und Hochschulbibliothek Luzern | Zentralbibliothek Zürich

Die Publikation entspricht den Qualitätsstandards für die Open-Access-Publikation von Büchern (Nationaler Open-Access-Kontaktpunkt et al. 2018), Phase 1 https://oa2020-de.org/blog/2018/07/31/empfehlungen_qualitätsstandards_oabücher/ $* * *$

Maximilian Pichl, geb. 1987, forscht im Rahmen eines Projektes der Hans-Böckler-Stiftung an der Universität Kassel zur Krise der EU-Migrationspolitik. Er studierte Rechtsund Politikwissenschaft in Frankfurt am Main. Zwischen 2015 bis 2017 war er rechtspolitischer Referent von PRO ASYL e.V. und ist im Vorstand vom Bundesfachverband unbegleitete minderjährige Flüchtlinge (BUMF e.V.).

Timo Tohidipur (Dr. jur.), geb. 1972, ist Professor am Fachbereich Soziale Arbeit und Gesundheit der Frankfurt University of Applied Sciences, Frankfurt am Main. Seine Forschungsschwerpunkte sind das Europäische Grenzregime, Migrationsrecht, Rechtsvergleichung und Recht im Film. 
Maximilian Pichl, Timo Tohidipur (Hg.)

\section{An den Grenzen Europas und des Rechts}

Interdisziplinäre Perspektiven auf Migration, Grenzen und Recht 


\section{Bibliografische Information der Deutschen Nationalbibliothek}

Die Deutsche Nationalbibliothek verzeichnet diese Publikation in der Deutschen Nationalbibliografie; detaillierte bibliografische Daten sind im Internet über http://dnb.d-nb.de abrufbar.

\section{(c) (1) ()}

Dieses Werk ist lizenziert unter der Creative Commons Attribution-ShareAlike 4.0 Lizenz (BY-SA). Diese Lizenz erlaubt unter Voraussetzung der Namensnennung des Urhebers die Bearbeitung, Vervielfältigung und Verbreitung des Materials in jedem Format oder Medium für beliebige Zwecke, auch kommerziell, sofern der neu entstandene Text unter derselben Lizenz wie das Original verbreitet wird. (Lizenz-Text: https://creativecommons.org/licenses/by-sa/4.0/deed.de)

Die Bedingungen der Creative-Commons-Lizenz gelten nur für Originalmaterial. Die Wiederverwendung von Material aus anderen Quellen (gekennzeichnet mit Quellenangabe) wie z.B. Schaubilder, Abbildungen, Fotos und Textauszüge erfordert ggf. weitere Nutzungsgenehmigungen durch den jeweiligen Rechteinhaber.

Erschienen 2019 im transcript Verlag, Bielefeld

(c) Maximilian Pichl, Timo Tohidipur (Hg.)

Umschlaggestaltung: Maria Arndt, Bielefeld

Druck: Majuskel Medienproduktion $\mathrm{GmbH}$, Wetzlar

Print-ISBN 978-3-8376-4714-3

PDF-ISBN 978-3-8394-4714-7

https://doi.org/10.14361/9783839447147

Gedruckt auf alterungsbeständigem Papier mit chlorfrei gebleichtem Zellstoff. Besuchen Sie uns im Internet: https://www.transcript-verlag.de

Bitte fordern Sie unser Gesamtverzeichnis und andere Broschüren an unter: info@transcript-verlag.de 


\section{Inhalt}

Vorwort | 7

Grenzbetrachtungen | 9

Sara Abbasi / Anna Hochleitner / Merve Kania / Lise Känner /

Thea Kirsch / Nils Imgarten / Kentaro Inagaki / Tuna Kaptan /

Maximilian Pichl / Timo Tohidipur / Mate Ugrin

\section{GRENZEN UND ZUGEHÖRIGKEIT}

Zur rhetorischen Verschiebung von Staatsgrenzen | 17

Lise Känner

\section{Zum Spannungsfeld}

zwischen Liberalismus und Migrationspolitik | 41

Kentaro Inagaki

\section{WIRTSCHAFT, RECHT UND POLITIK DER MIGRATION}

Eine ökonomische Perspektive auf Wanderungsbewegungen und Migrationspolitik | 59 Anna Hochleitner

Der Bedarf nach einem neuen Einwanderungsrecht | 91 Eine Analyse aus juristischer Perspektive

Nils Imgarten

Eine soziohistorische Perspektive auf Deutschlands Migrationsreformen | 119

Un-/erwünschte Mobilität und die Debatte um ,Wirtschaftsflüchtlinge،

Merve Kania 


\section{AN DEN GRENZEN DES RECHTS}

Das Visum und die Verlagerung der Grenzkontrolle | 149 Thea Kirsch

Verpflichtung zur aktiven Seenotrettung | 177

Zu den nicht intendierten Effekten der Überwachung des Mittelmeers durch Frontex Maximilian Pichl / Timo Tohidipur

\section{LEBEN NACH DER GRENZE}

How social media facilitate migration in Europe | 211 Sara Abbasi

Waiting Room. A Short Essay Film | 221

Tuna Kaptan / Mate Ugrin

Autorinnen und Autoren | 225 


\section{Vorwort}

Der Sammelband ist das Ergebnis einer zweijährigen Zusammenarbeit der Autor/innen im Kolleg Europa. Das Kolleg Europa ist eine Kooperation zwischen der Studienstiftung des deutschen Volkes, dem DAAD und der Alfred-Töpfer-Stiftung. Zwischen 2016 und 2018 fanden in diesem Rahmen insgesamt vier jeweils einwöchige Seminare mit insgesamt 70 Stipendiat/innen und Dozent/innen statt, die sich in verschiedenen Arbeitsgruppen unter dem Schlagwort „Europa offen denken“ zusammenfanden. Die einzelnen Kollegwochen wurden an Orten veranstaltet, die sich aus unterschiedlichen Gründen für eine Auseinandersetzung mit Grenzen angeboten haben und an denen Stipendiat/innen mit politischen und zivilgesellschaftlichen Akteuren zusammentreffen konnten. Slubice (Polen) eignete sich als Auftakt, weil sich die Stadt in unmittelbarer Nachbarschaft zu Frankfurt/Oder befindet und wie keine andere für die Auflösung von Grenzen, Zäunen und Kontrollen steht. In Budapest (Ungarn) vollzieht sich hingegen aktuell eine neue Form der europäischen Abschottungspolitik, die sich in dem von der Regierung Viktor Orbáns errichteten Zaun zwischen Ungarn und Serbien zeigt. Paris (Frankreich) und schließlich Berlin (Deutschland) stehen für Städte, in denen alläglich die Chancen und Herausforderungen einer postmigrantischen Gesellschaft auf je verschiedene Weise ausgehandelt werden.

Die Beiträge entstanden in der stipendiatischen Arbeitsgruppe „Wie offen sind Europas Grenzen? Migration und Flucht aus rechtlicher Perspektive“, die im Rahmen des Kolleg Europa von Timo Tohidipur und Maximilian Pichl geleitet wurde, unter der Mitwirkung von Sara Abbasi, Anna Hochleitner, Merve Kania, Lise Känner, Thea Kirsch, Nils Imgarten, Kentaro Inagaki, Tuna Kaptan und Mate Ugrin. 
Johannes Klein und Lukas Schmidt waren ebenfalls Teil der KollegGruppe, konnten jedoch aus unterschiedlichen Gründen keinen eigenen Beitrag für den Sammelband einreichen. Sie waren aber an allen Diskussionen beteiligt und ihre Perspektiven sind daher auch in die Beiträge miteingeflossen. Die Beiträge wurden gemeinsam von den Stipendiat/innen im Rahmen der Kollegwochen und zwei Zwischentreffen in Dresden und Brüssel intensiv diskutiert und anschließend ausgearbeitet.

An dieser Stelle möchten sich die Herausgeber und die Stipendiat/innen ausdrücklich bei Dr. Lukas Werner, Cathrin Anderwaldt und Dr. Valeska Bopp-Filimonov von der Studienstiftung des deutschen Volkes bedanken, die aufgrund ihrer organisatorischen Arbeit überhaupt erst den Rahmen für die produktiven Diskussionen während der Kollegwochen geschaffen haben.

Die Studienstiftung des deutschen Volkes hat den überwiegenden Anteil der Kosten für dieses Buchprojekt übernommen, weshalb die Herausgeber und Stipendiat/innen an dieser Stelle einen großen Dank aussprechen. 


\title{
Grenzbetrachtungen
}

\author{
Sara Abbasi / Anna Hochleitner / Merve Kania / Lise Känner / \\ Thea Kirsch / Nils Imgarten / Kentaro Inagaki / Tuna Kaptan / \\ Maximilian Pichl / Timo Tohidipur / Mate Ugrin
}

\section{INTERDISZIPLINÄRE PERSPEKTIVEN AUF DIE GRENZE}

Die Kategorie der Territorialität und damit auch die Kategorie der Grenze, die schon begriffsgeschichtlich einen territorialen Einschnitt definiert, verliert im Zuge der Globalisierung nur vermeintlich an Relevanz, während zugleich die Frage nach den Zugangsbedingungen von Menschen zu politischen Räumen, seien sie national oder supranational definiert, an Bedeutung gewinnt. Dabei gehören Grenzen, zunächst unabhängig vom disziplinären Kontext, zu den Konstanten menschlichen Denkens und Handelns. ${ }^{1}$ Als Rechtsvorschriften regulieren Grenzen nach einer In/out-Systematik soziale Handlungsbereiche, staatliche oder überstaatliche Zugriffsmöglichkeiten sowie Zuordnungen von Rechten und Pflichten.

Rechtlich gesetzte Grenzen sind Konstanten der zeitgenössischen politisch-rechtlichen Staatenrealität. Zugleich sind sie aber auch der (teilweise imaginierte) Ort, an dem das Versprechen auf steuerbare Migration umgesetzt werden soll: Neben den physischen sind es vor allem auch diese abstrakten Grenzen, die den Zuzug nach Europa bestimmen.

Obwohl Wanderungsbewegungen seit jeher Teil der Menschheitsgeschichte waren und auch zu tiefgreifenden gesellschaftlichen Veränderun-

1 Kleinschmidt, Christoph, Semantik der Grenze, APuZ 4-5/2014, S. 3. 
gen geführt haben, ${ }^{2}$ führen erleichterte Informationsgewinnung über Beschäftigungs- und Lebensaussichten in anderen Regionen im Zusammenspiel mit einer enormen weltweiten Ungleichheit der Lebensbedingungen zu einer verstärkten Migrationsentwicklung. Hinzu treten u.a. langfristige Umweltveränderungen und die Zunahme von militärischen Konflikten an der Peripherie der Europäischen Union (Nordafrika, Westasien), die ebenfalls Wanderungsbewegungen bzw. Fluchtursachen generieren. ${ }^{3}$ Die fortschreitende Globalisierung wirkt sich damit auch auf die transnationalen Grenzregime aus. Staaten oder Staatenkooperationen, die versuchen, Migration zu regulieren oder gar ihr Staatsgebiet betreffend zu unterbinden, sehen sich vor neue Probleme gestellt, wie gerade die Krise des Flüchtlingsschutzes seit 2015 verdeutlicht. ${ }^{4}$

In der öffentlichen Wahrnehmung spielt auch ein verändertes Gefühl für die Beständigkeit der bestehenden eigenen Staatsgrenzen eine Rolle. Die europäischen sowie weitere Staaten, die gemeinhin dem „Westen“ zugeordnet werden, erfahren aktuell die längste anhaltende Friedensphase ihrer Geschichte. Gleichzeitig verfestigte sich der völkerrechtliche Grundsatz der Souveränität der Staaten, welcher die territoriale Unversehrtheit eines Hoheitsgebietes in den Mittelpunkt politischer und rechtlicher Garantien stellt. Beides stärkt das gesellschaftliche und politische Gefühl einer (vermeintlichen) Bestandskraft der Staatsgrenzen mit Ewigkeitsgarantie. Das Bedürfnis, eine solche für das Bewahren der bestehenden sozialen Ordnungen zentrale Einrichtung zu schützen, wird durch diese noch jungen historischen Prägungen der ,,westlichen“ Gesellschaften verstärkt. Dieses Bedürfnis wird begleitet von der Wahrnehmung einer ausgeprägten Bedrohungslage durch Migration als solche, da diese innerhalb dieses Denkmusters die beiden oben beschriebenen Erfahrungen in Frage zu stellen vermag.

Migrationsbewegungen und Bestrebungen, ebenjene aufzuhalten, weisen im Regelfall für beide beteiligten Parteien eine hohe existenzielle Bedeutung auf. Während es für die migrierenden Menschen im schlimmsten Fall wortwörtlich um ihr Leben gehen kann, sieht sich ein Staat möglicherweise verpflichtet, seine soziale und (häufig) demokratische Ordnung zu

2 Oltmer, Jochen, Globale Migration, 3. Aufl. 2016, S. 7.

3 Oltmer, Jochen, Globale Migration, 3. Aufl. 2016, S. 123 ff., 128.

4 Cuttitta, Paolo, Das Europäische Grenzregime: Dynamiken und Wechselwirkungen, in: Hess/Kasparek (Hrsg.), Grenzregime, 2010, S. 23. 
erhalten. Aus Sicht der Staaten geht es auch hier um das Bewahren ihrer traditionellen Existenzbedingungen. Die Migrationsfrage ist also zugleich eine menschenrechtliche, soziale und demokratische Frage. Dies zeigt auf, dass die aufgrund widerstreitender Interessen an der Staatsgrenze entstehenden Konflikte hohe menschenrechtliche Implikationen aufweisen, die unmittelbar das Paradox liberaler Gesellschaften sichtbar machen, die zwischen Inklusion und Exklusion von Migration oszillieren. Migration kann deshalb je nach Perspektive sowohl als Garant als auch als Bedrohung menschenrechtlicher Errungenschaften verstanden werden.

Der Umgang mit Migration ist auch für die Europäische Union ein grundlegendes Thema, denn die Ausgestaltung des grundsätzlich grenzfreien Binnenmarktes ist abhängig von der Reichweite der Solidarität innerhalb der Europäischen Union und dem Verhältnis zur eigenen souveränen Staatlichkeit der Mitgliedstaaten. Die Europäische Union hat ein umfangreiches Regelwerk zur Steuerung und Regulierung von Migration etabliert, das mitgliedstaatliche Regeln zu Einwanderung und Grenzschutz ergänzt und in Teilen überformt. Gerade der europarechtliche Vorrang sorgte in den letzten Jahren für politische Verwerfungen zwischen denjenigen, die eine gesamteuropäische Lösung präferieren und jenen die auf die vermeintlich ungeteilte nationalstaatliche Souveränität in Migrationsfragen setzen wollen. Auch die EU-Institutionen müssen sich angesichts der oben beschriebenen menschenrechtlichen Implikationen der Regulierung von Migration einer kritischen Prüfung unterziehen lassen, inwieweit die bestehenden Regeln menschenwürdige Migration erschweren oder verhindern. Darauf aufbauend lässt sich auch allgemeiner fragen, ob die EU so den eigenen normativen Grundlagen und Ansprüchen noch gerecht wird. Zudem stehen zuweilen gerade solchen europarechtlichen Vorgaben, die Migration - sei sie regulär oder irregulär - flankieren und die Rechte Einzelner rechtlich untermauern sollen, mitgliedstaatliches Recht oder fehlende Ressourcen für eine praktische Umsetzung entgegen. Auch die einschlägigen völkerrechtlichen Normen wie z.B. die Genfer Flüchtlingskonvention oder das UN-Seerechtsübereinkommen werden nicht selbstverständlich eingehalten, vielmehr muss ihre Durchsetzung fortwährend politisch und rechtlich erkämpft werden.

Die vielgestaltigen Rechtsgrundlagen sind jedoch nur ein Pfeiler des Umgangs der Europäischen Union und ihrer Mitgliedstaaten mit Grenzen und den daran anknüpfenden Zugangsbedingungen. Für ein breiteres Ver- 
ständnis ist die Einbeziehung der politologischen, soziologischen, ökonomischen, journalistischen und künstlerischen Dimensionen überaus relevant. Erst in der Zusammenschau dieser disziplinübergreifenden Perspektiven lassen sich die Handlungsformen und -möglichkeiten der unterschiedlichen Akteur*innen und auch der Rechts- und Lebensstatus der Betroffenen verstehen.

\section{AUFBAU DES SAMMELBANDS}

Der vorliegende Sammelband versucht, sich dieser Gemengelage von Herausforderungen und Entwicklungen im Zusammenhang von Grenze und Migration aus unterschiedlichen (inter)disziplinären Perspektiven zu nähern. Dabei wird ein Bogen gespannt von theoretischen Erwägungen bis hin zu den konkreten Lebenserfahrungen von Migrierenden an und nach der Grenze.

Die komplexe Form der Verrechtlichung der Grenzen gilt es aus verschiedenen Zugängen heraus zu erarbeiten. Der Band dokumentiert daher zugleich einen Dialog zwischen den Disziplinen. Auf eine einheitliche Zitierweise wurde verzichtet, um die wissenschaftliche Interdisziplinarität auch formal sichtbar zu machen.

Lise Känner widmet sich in ihrem Beitrag den rhetorischen Verschiebungen von Staatsgrenzen und zeigt auf, dass die vermeintlich statische (Staats-)Grenze selbst unter den geltenden Bedingungen des Rechts aufgrund von politischer Rhetorik ihre tatsächliche Eindeutigkeit verliert und je nach Bedarf der verantwortlichen Akteure sprachlich neu gezogen wird. Sie arbeitet die hohe Relevanz des tatsächlichen wie behaupteten Grenzverlaufs für den Zugang zum Recht heraus und legt dar, dass gerade in Fluchtsituationen an der Grenze systematisch Rechtsbrüche betrieben werden.

Kentaro Inagaki betrachtet das Spannungsverhältnis von Exklusion und Inklusion im Liberalismus. Er zeigt am Beispiel des Vorschlags, Flüchtlingen die europäische Unionsbürgerschaft zu verleihen, wie das Paradox von Inklusion und Exklusion strukturell im Recht verankert ist und aktuell von jeweils anderen politischen Strömungen mobilisiert wird.

Anna Hochleitner hinterfragt in ihrer ökonomischen Perspektive auf Wanderungsbewegungen und Migrationspolitik kritisch die vermeintlich belastenden Auswirkungen von Migration auf die Arbeitsmarktbedingun- 
gen und den Sozial- bzw. Wohlfahrtstaat. Sie zeigt auf, dass öffentliche Wahrnehmung und volkswirtschaftliche Realitäten auch in diesem Kontext nicht notwendig übereinstimmen.

Nils Imgarten setzt sich ausgehend von einer Systematisierung des deutschen und europäischen Migrationsrechts mit der Möglichkeit der Schaffung legaler Zugangswege als Strategie zur Verringerung irregulärer Migration auseinander. Dabei nimmt er insbesondere aktuell diskutierte Vorschläge und Ansätze für ein Einwanderungsgesetz auf, stellt sie der bestehenden Rechtslage gegenüber und diskutiert alternative Reformvorschläge für ein neues Migrationsrecht auf nationaler und europäischer Ebene.

Merve Kania situiert die Debatten um ein Einwanderungsgesetz in einem historischen Kontext. Sie zeigt anhand einer Auswertung von Bundestagsdebatten, wie die Figur des „Wirtschaftsflüchtlings“ entstanden ist und damit einhergehend Diskurse um den Topos der „Sicherheit“ in der Migrationspolitik wirkmächtig wurden.

Thea Kirsch widmet sich der globalen und insbesondere europäischen Visumpolitik. Sie zeigt anschaulich auf welche Art und Weise das Visum zu dem kennzeichnenden Moment geworden ist, der über die globale Mobilität von Menschen entscheidet - und wie die selektive Gewährleistung von Rechten die ungleiche Macht- und Chancenverteilung auf der Welt verschärft.

Maximilian Pichl und Timo Tohidipur unterziehen die Praxis der Seenotrettung der Mitgliedstaaten und der EU im Lichte europäischer und völkerrechtlicher Normen einer kritischen Analyse. Die seitens der Agentur für Grenz- und Küstenwache (Frontex) angestrebte, umfassende Überwachung des Mittelmeerraums führt ihnen zufolge zu dem nicht intendierten Effekt, dass für sämtliche durch die Überwachung identifizierten Boote in Seenot eine Rettungspflicht begründet wird.

Sara Abbasi untersucht die Erfahrungen von Migration und dem Leben nach der Grenze aus einer journalistischen Perspektive und hinterfragt insbesondere, inwiefern soziale Medien Migration und Ankommen verändern.

Tuna Kaptan und Mate Ugrin haben während der Kollegwochen einen Film über die Ausnahmesituation vom Sommer 2015 am Budapester Bahnhof Keleti gedreht. Sie reflektierten in diesem Sammelband kurz über den Hintergrund des Films. Ein Zugangscode für den Film ist dem Sammelband beigefügt. 

Grenzen und Zugehörigkeit 



\section{Zur rhetorischen Verschiebung von Staatsgrenzen}

\section{Lise Känner}

\section{A. EINLEITUNG}

Staatsgrenzen regeln territoriale Markierungen zur Absicherung von Macht, an denen der Hoheitsbereich des einen Staates endet und der eines anderen beginnt. ${ }^{1}$ Die Grenze befindet sich im Spannungsfeld des Bedürfnisses nach (vermeintlicher) Sicherheit einerseits und der Gewährung von Schutz vor Krieg und Verfolgung andererseits. Sie ist somit Mittelpunkt der politischen Auseinandersetzungen im aktuellen öffentlichen Diskurs um Sicherheit und Verantwortung: Gegenstand dieser Auseinandersetzungen sind die seitens der EU gegenüber einigen Mitgliedstaaten sogar geforderte, partielle Durchsetzung der Wiedereinführung von Grenzkontrollen im Schengenraum, ${ }^{2}$ der Bau von Grenzzäunen an den EU-Außengrenzen und der Einsatz von militärischen Mitteln zur Abwehr von irregulärer Migration. ${ }^{3}$

Dem tatsächlichen Grenzverlauf kommt dabei für die Bewertung von Amtshandlungen von Grenzschutzbeamten und -beamtinnen mit asyl- und

1 Kleinschmidt, Semantik der Grenze, in: APuZ 4-5/2014, S. 3.

2 Council of the EU, Implementing Decision (EU) 2017/818 of 11 May 2017, setting out a Recommendation for prolonging temporary internal border control in exceptional circumstances putting the overall functioning of the Schengen area at risk, Amtsblatt EU, L 122/73.

3 Council of the EU, Decision (CFSP) 2016/993 of June 2016 zur Militäroperation EUNAVFOR MED Operation Sophia, AB1. L 162/18. 
migrationsrechtlichem Bezug elementare Bedeutung zu. Nur wenn der Grenzverlauf eindeutig zugeordnet werden kann, können wichtige Anschlussfragen wie die nach der rechtlichen Zuständigkeit aneinandergrenzender Staaten für das Entgegennehmen des Gesuchs nach Asyl beantwortet werden. Die Versteifung auf eine rechtliche Beschreibung des Grenzverlaufs allein wird allerdings der Realität nicht mehr gerecht. Dieser Beitrag wird zeigen, dass in mehreren Fällen aufgrund von zeitlich vor- oder nachgelagerten Aussagen und Anweisungen von politischen Entscheidungsträgern in der Praxis von dem rechtlichen Verlauf der Grenze abgewichen wurde, um bestimmte rechtswidrige Vorgehensweisen zu legitimieren.

Es ist außerdem These dieses Beitrages, dass diese Vorkommnisse keine Einzelfälle sind. Vielmehr handelt es sich um eine systematische Entleerung des rechtlichen Begriffs der Staatsgrenze, welche zu einem zunehmenden Bedeutungs- und Geltungskraftverlust der rechtlichen Beschreibung des Grenzverlaufs sowie zu erheblichen asyl- und migrationsrechtlichen Folgeproblemen führt.

\section{B. DIE RECHTLICHE BESCHREIBUNG DER GRENZE}

Zunächst ist für die Untersuchung des Phänomens der rhetorischen Grenzverschiebung eine genaue rechtliche Beschreibung der Staatsgrenze notwendig. Nur dann kann die Diskrepanz zwischen in der Praxis gelebter und rechtlicher Grenzziehung akkurat beschrieben werden. Außerdem begrenzt sich dieser Beitrag begrifflich aufgrund seines Formates auf Landgrenzen. ${ }^{4}$

\section{Rechtspraxis der Grenzziehung und -änderung}

Ausgangspunkt der Überlegung, wie Grenzen gezogen werden, ist die Souveränität der Staaten. Dieses System geht zurück auf den Westfälischen Frieden 1648, der das Staatssystem nicht auf Grundlage von Macht, sondern auf der Basis rechtlicher Gleichordnung festschrieb. Dies umfasste auch das Recht der Grenzziehung: Nur der souveräne Staat sollte seine Grenzen und damit sein Territorium und den Zugang zu selbigem festlegen

$4 \mathrm{Zu}$ der spezifischen Problematik im Zusammenhang mit Seegrenzen siehe den Beitrag von Maximilian Pichl und Timo Tohidipur in diesem Band. 
können. ${ }^{5}$ Unzweifelhaft kann dieses Vorgehen bei benachbarten Staaten zu Konflikten führen, soweit sich diese über den Grenzverlauf nicht einig sind. Solche Konflikte werden im Regelfall durch bi- oder multilaterale Verträge beigelegt. Ihnen werden Koordinatenbeschreibungen sowie Karten angehängt, die den genauen Grenzverlauf verbindlich festlegen. ${ }^{6}$ Darüber hinaus werden die Grenzverläufe eines Staates dann auch faktisch international verfestigt, wenn die anderen Staaten ihn bei der UN offiziell anerkennen. Durch die Anerkennung als souveräner Staat werden auch dessen hinterlegte Grenzverläufe anerkannt.

Nachträgliche Änderungen des Grenzverlaufes werden regelmäßig ebenfalls durch bi- oder multilaterale Verträge von den beteiligten Staaten festgelegt. Fraglich ist, ob eine Änderung der Staatsgrenzen auch durch Gewohnheitsrecht möglich ist. Voraussetzungen für die Annahme von Gewohnheitsrecht ist völkerrechtlich mindestens eine unwidersprochene Staatenpraxis. $\mathrm{Ob}$ dies in den hier besprochenen Fällen zum Tragen kommen kann, erscheint bereits auf den ersten Blick zweifelhaft, eine nähere Betrachtung soll unter Kapitel D.I. (Auswirkungen der rhetorischen Verschiebung für die juristische Beschreibung) vorgenommen werden. ${ }^{7}$

\section{Funktionen der rechtlichen Beschreibung der Grenze}

Den oben beschriebenen Landgrenzen kommen verschiedene Funktionen im Staatsgefüge zu. Die erste und wichtigste Funktion der Staatsgrenze ist,

5 Vgl. eingehend Kahn, Territory and Boundaries, in: Fassbender/Peters (Hrsg.), The Oxford Handbook of The History of International Law, 2012, S. 225 (233 ff.).

6 Vgl. zum Beispiel den Vertrag über den Verlauf eines Abschnittes der deutschösterreichischen Grenze von 1974: BGBl. Nr. 388/1979; s. ausführlich Krajewski, Völkerrecht, 2017, § 7 Rn. 17 ff.

7 Letztlich darf Gewohnheitsrecht im Völkerrecht auch nicht sog. ,ius cogens“, also zwingendes Recht in Frage stellen. Es erscheint allerdings möglich, dass zu den Grundsätzen des ius cogens auch die Souveränität der Staaten liegt. Wie oben bereits gezeigt ist Ausfluss dieses Prinzips auch das Recht, die eigenen Grenzen alleinig zu bestimmen. Ob dieses tatsächlich lediglich durch eine von der Rechtslage abweichenden Staatspraxis angetastet werden darf, erscheint äußerst fraglich. 
festzulegen, auf welchem Gebiet welche Hoheitsmacht herrscht. Sie legt fest, wo der politische Körper A regiert und wo Körper B. Damit legt die Staatsgrenze primär die Zuständigkeiten der beteiligten Akteure und Akteurinnen fest.

Zudem kommt der Staatgrenze in konkreten Fällen - in Fortführung der Überlegungen zur Zuständigkeit - die Funktion zu, den Zugang zum Recht zu regeln. Allgemein bedeutet dies, dass sie bestimmt, auf welchem Gebiet welche Rechtsnormen für wen gelten. In den für diesen Beitrag relevanten Fällen geht es im Speziellen um den Zugang zu rechtsstaatlichen Asylverfahren sowie zu innerstaatlichem gerichtlichen Rechtsschutz. Gem. Art. 18, 19 GrCh, Art. 4 des 4. Zusatzprotokolls zur EMRK und Art. 3 I Dublin-IIIVO sind (europäische) Staaten verpflichtet, jeder Person, die ihr Staatsgebiet betritt, die Möglichkeit zu geben, um Asyl zu ersuchen. Hiervon ausgehend ist der Grenzverlauf für Geflüchtete von elementarer Bedeutung: So lange sie die Grenze noch nicht überquert haben, können sie kein Asylgesuch stellen. Ganz konkret kann Schutz vor Verfolgung und Krieg überhaupt nur durch Staaten und damit nur dann geleistet werden, wenn die Grenze aus Sicht des oder der Schutzsuchenden überhaupt übertretbar ist. ${ }^{8}$ Daran anschließend ist auch die Möglichkeit, gerichtlichen Rechtsschutz in Anspruch zu nehmen, davon abhängig, ob bestimmte Handlungen auf dem inländischen Staatsgebiet vorgenommen wurden oder nicht.

\section{Offenheit der rechtlichen Beschreibung für den rechtspolitischen Diskurs}

Die rechtliche Beschreibung von Sachverhalten ist kein starres, unveränderliches Gefüge. Rechtsbegriffe sind offen für Interpretation und stets im Kontext gesellschaftlicher Aushandlungsprozesse zu verstehen. ${ }^{9}$ Grundsätzlich bedeutet dies, dass das, was einmal mit der Setzung einer Rechtsnorm bezweckt wurde, nicht zwangsläufig 20 Jahre später in der gleichen Form so auch noch gelten muss. Dies ist allerdings nur bedingt auf die Rechtskraft der (Staats-)Grenze übertragbar. So bleibt bei der rechtlichen Beschreibung einer Linie wenig bis gar kein Raum für alternative Auslegun-

8 Siehe hierzu auch den Beitrag von Thea Kirsch.

9 Statt vieler s. Rüthers/Fischer/Birk, Rechtstheorie, 10. Auflage 2018, S. 101 ff., insb. S. 109 ff. m.w.N. 
gen. Selbst eine Veränderung der gesellschaftlichen Wahrnehmung einer Grenze kann nicht dazu führen, dass sich die im Grenzvertrag festgelegten Koordinaten für den Grenzverlauf ändern. Insofern ist der Grenzverlauf an sich für keine anderweitige Interpretation offen, die sog. Wortlautgrenze ist absolut.

\section{DIE POLITISCHE BESCHREIBUNG DER GRENZE}

Im Gegensatz zur rechtlich eindeutigen Beschreibung des Grenzverlaufs tragen politische Entscheidungsträger*innen zur öffentlichen Wahrnehmung der Grenze bei, indem sie das Verhalten von Grenzbeamten anweisen oder die Ausgestaltung von Handlungsspielräumen nachträglich bewerten. Sie machen durch den Ausspruch selbst und die daraus abgeleiteten Handlungen der Grenzschutzbeamt*innen die Grenze erst sichtbar. ${ }^{10}$

\section{Beschreibung durch politische Entscheidungsträger*innen}

Rechtstreue Entscheidungsträger*innen sollten sich bei der politischrhetorischen Beschreibung uneingeschränkt an der rechtlichen Beschreibung des Grenzverlaufs orientieren. Sie müsste es öffentlich verurteilen, wenn die ihnen unterstellten Beamt*innen auf ihrem Hoheitsgebiet Geflüchtete in den Nachbarstaat zurückdrängen, ohne ihnen die Möglichkeit zu geben, ein Asylgesuch zu stellen.

In der Praxis sieht das anders aus. Es häufen sich Fälle, in denen in offiziellen Anweisungen an die handelnden Beamt*innen vor Ort oder bei nachträglichen Bewertungen von Vorgängen die politisch Verantwortlichen einen Grenzverlauf darstellen, der mit dem rechtlichen nicht übereinstimmt. Dieser behauptete Verlauf legitimiert dann ein unter Umständen rechtswidriges Verhalten der Beamt*innen. Im Folgenden soll auf einige Beispiele eingegangen werden.

10 Vgl. grundsätzlich zur Bedeutung von Rhetorik im rechtswissenschaftlichen Diskurs um Sicherheitspolitik sowie verschiedene Methoden der Diskursanalyse Röhner, Sicherheit als rechtswissenschaftliche Praxis, in: KritV 98 (2015), S. $153 \mathrm{ff}$. 


\section{Melilla}

Die spanische Exklave Melilla liegt auf afrikanischem Festland und grenzt an Marokko. Dort wurden drei hintereinanderstehende, mehrere Meter hohe Zäune gebaut, um Geflüchtete vom Betreten des spanischen Staatsgebietes abzuhalten. Die Zaunanlage steht jedenfalls teilweise auf spanischem Staatsgebiet, ${ }^{11}$ wird jedoch insgesamt von spanischen Beamt*innen verwaltet. ${ }^{12}$ Von 2005 bis 2015 wurde die Guardia Civil administrativ und ohne gesetzliche Grundlage angewiesen, Geflüchtete, die versuchten, die Zaunanlage zu überwinden, direkt zurück auf marokkanisches Staatsgebiet zu verbringen. ${ }^{13}$ Dabei war die offizielle Aussage der spanischen Regierung, dass zusätzlich zur Staatsgrenze noch eine sog. operative Grenze (die Zaunanlage) überwunden werden müsse, um einen Antrag auf Asyl stellen zu können. Seit 2015 wurde diese Praxis mit einer gesetzlichen Grundlage

11 Die spanische Regierung bestreitet dies entgegen der den Grenzverlauf festlegenden internationalen Verträge, vgl. N.D and N.T.. v. Spain, EGMR, Urteil vom 03.10.2017, Az.: 8675/15 und 8697/15, Rn. 17 und 44 f., während das European Center for Constitutional and Human Rights (ECCHR) sowie einige Third Party Interveners davon ausgehen, dass der Zaun in seiner Gesamtheit auf spanischem Territorium steht: ECCHR, Fallbeschreibung: Die spanisch-marokkanische Grenze bei Melilla - Push-Backs im rechtsfreien Raum, abrufbar: https://www. ecchr.eu/de/unsere-themen/voelkerstraftaten-und-rechtliche-verantwortung/mi gration-und-flucht/melilla.html?file=tl_files/Dokumente/Universelle\%20Justiz/ Fallbeschreibung_Melilla_EGMR_20171003.pdf (Entnahme: 02.11.2018); N.D and N.T.. v. Spain, EGMR, Urteil vom 03.10.2017, Az.: 8675/15 und 8697/15, Rn. $45 \mathrm{ff}$.

12 ECCHR, Fallbeschreibung, S. 2, abrufbar: https://www.ecchr.eu/de/unserethemen/voelkerstraftaten-und-rechtliche-verantwortung/migration-und-flucht/ melilla.html?file=tl_files/Dokumente/Universelle\%20Justiz/Fallbeschreibung Melilla_EGMR_20171003.pdf (Entnahme: 02.11.2018).

13 Dabei lassen die spanischen Beamten regelmäßig marokkanische Sicherheitsbeamte die Geflüchteten aus der Zaunanlage holen. Es wird berichtet, dass die marokkanischen Beamten die betreffenden Personen bei diesem Vorgehen und danach massiv misshandeln, vgl. ebd. S. 1; ebenfalls dokumentiert im Fall N.D. v. Spain, EGMR, Urteil vom 03.10.2017, Az.: 8675/15, Rn. 12. 
versehen, die die unterschiedslosen Abschiebungen aus der Zaunanlage zurück auf marokkanisches Staatsgebiet formal legalisiert haben. ${ }^{14}$

Bei der von den spanischen Behörden postulierten „operativen“ Grenze handelt es sich nicht um eine Staatsgrenze in dem oben beschriebenen Sinne. Vielmehr stellt sie eine funktionale Grenze dar. Die Verwender dieser Bezeichnung spielen hier bewusst mit dem Vokabular. Dadurch, dass sie den Begriff der Grenze verwenden, suggerieren sie, es handele sich auch um eine klare rechtliche Grenzsituation. Im öffentlichen Bewusstsein wird ein Zaun, eine Mauer oder ein Grenzposten aber regelmäßig mit der Staatsgrenze gleichgesetzt; optische Erkennungszeichen visualisieren eine Grenze erst. Gleichzeitig suggerieren solche Erkennungszeichen ebenfalls, es handele sich um eine rechtlich korrekte Beschreibung.

Zudem kommt es ebenfalls immer wieder zu der Situation, dass Geflüchtete, die weit in die Zaunanlage vorgedrungen sind, von den spanischen Behörden in Melilla inhaftiert werden und anschließend unterschiedslos abgeschoben werden. ${ }^{15}$ Auch hier zeigt sich, wie widersprüchlich das Verhalten der spanischen Behörden ist. Verhaften kann man nämlich jemanden nur, wenn es hierfür eine Rechtsgrundlage, also in den meisten Fällen einen Rechtsverstoß seitens des Verhafteten gegeben hat. Dies ist aber nur denkbar, wenn überhaupt Recht gilt, gegen das verstoßen werden könnte. Gerade das bestreiten die Behörden aber ja für den Bereich der Grenzzaunanlage. Es ist daher davon auszugehen, dass die spanischen Behörden selektiv von einer Geltung des Rechts innerhalb der Zaunanlage nur

14 ECCHR, Fallbeschreibung, S. 2 ff., abrufbar: https://www.ecchr.eu/de/unserethemen/voelkerstraftaten-und-rechtliche-verantwortung/migration-und-flucht/ melilla.html?file=tl_files/Dokumente/Universelle\%20Justiz/Fallbeschreibung_ Melilla_EGMR_20171003.pdf (Entnahme: 02.11.2018).

15 So auch im Fall N.D. v. Spain, EGMR, Urteil vom 03.10.2017, Az.: 8675/15, Rn. 12. 
dann ausgehen, wenn es für sie einen Vorteil bringt. Insgesamt handelt es sich schlicht um Willkür. ${ }^{16}$

\section{Ungarn/Serbien}

Die ungarische Regierung begann 2015 mit dem Bau eines Zaunes an der ungarisch-serbischen Grenze. Diese EU-Außengrenze lag insbesondere in den Jahren 2014 und 2015 auf einer der Hauptrouten von Geflüchteten auf dem Weg nach Mittel- und Westeuropa. Die Regierung unter Viktor Orbán bezweckte mit dem Bau des Zaunes die deutliche Reduzierung der Zahl der Geflüchteten, die in Ungarn und der EU insgesamt ankamen. Der Grenzzaun wurde ebenfalls auf ungarischem Staatsgebiet errichtet und mit zwei baulichen Transitanlagen (Transitzonen) versehen, die pro Werktag nur jeweils einen Mensch durchlassen. ${ }^{17}$ Komplementär hierzu wurden die Grenzbeamt*innen angewiesen, innerhalb eines Abstandes von acht Kilometern hinter dem Grenzzaun auf ungarischer Seite aufgefasste Geflüchtete

16 Diese ist auch bereits für sich betrachtet rechtswidrig. Das Willkürverbot wird zumeist rezipiert in gleichheitsrechtlichen Vorschriften sowie in Diskriminierungsverboten. Letztlich kann auch die Verpflichtung auf den Verhältnismäßigkeitsgrundsatz als Ausfluss mindestens des Willkürverbots verstanden werden. Im deutschen Recht leitet sich der allgemeine Grundsatz des Willkürverbots aus Art. 3 I GG sowie dem Rechtsstaatsprinzip insgesamt ab, vgl. BVerfGE 86, 148 (251); E 69, 161 (168 f.). Dabei wird eine Entscheidung als willkürlich verstanden, die nicht mehr verständlich ist und auf sachfremden Erwägungen beruht, st. Rspr. des BVerfG seit BVerfG 4, 1 (7). In der EMRK sei insbesondere auf Art. 1 Abs. 2 des 12. Zusatzprotokolls verwiesen, der jegliche Diskriminierung von Bürgern durch Behörden untersagt. Grundlegend zur Bedeutung des Willkürverbots im Völkerrecht siehe bereits Leibholz, Das Verbot der Willkür und des Ermessensmißbrauches im völkerrechtlichen Verkehr der Staaten, ZaöRV 1929, S. $77 \mathrm{ff}$.

17 Kolb, Matthias, Ein Land schottet sich ab, 16.06.2018, https://www.sueddeu tsche.de/politik/fluechtlinge-in-ungarn-ein-land-schottet-sich-ab-1.4014923 (Ent nahme: 02.11.2018); zum Vergleich: 2017 waren es noch ca. 15 Menschen pro Tag, Helsinki Committee Hungary (Hrsg.), Pushed Back at the Door: Denial of Access to Asylum in Eastern EU Member States, 2017, S. 12 ff., abrufbar: https://www.proasyl.de/wp-content/uploads/2015/12/pushed_back.pdf (Entnahme: 02.11.2018). 
wieder auf die andere Seite des Zaunes in Richtung Serbien zu verbringen. ${ }^{18}$ Hierdurch sollte sichergestellt werden, dass Geflüchtete Ungarn ausschließlich durch die Transitzone betreten können. Anfang 2017 verabschiedete die Regierung gemeinsam mit dem Parlament ein Gesetz, welches es den Behörden erlaubt, Geflüchtete, deren Asylverfahren noch nicht abgeschlossen ist, unabhängig von ihrem Aufenthaltsort in Ungarn auf die andere Seite des Zauns bzw. in die nun deutlich vergrößerten Transitzonen $\mathrm{zu}$ verbringen und dort zeitlich unbegrenzt zu inhaftieren. ${ }^{19}$ Dabei traten Orbán sowie andere Angehörige seiner Regierung in den vergangenen Monaten und Jahren immer wieder mit Äußerungen in Erscheinung, die die Bedeutung des effektiven Grenzschutzes für die „,völkische Reinheit der Magyaren“ ${ }^{620}$ betonen. $^{21}$ Rechtlich stellt sich die Regierung auf den Standpunkt, dass hinter dem Grenzzaun noch einige Meter ungarisches Staatsgebiet sei und die Abschiebungen auf die andere Seite des Zaunes somit keine illegalen Push-Backs darstellten. ${ }^{22}$

18 Ebd.

19 Vgl. PRO ASYL, Inhaftierung aller Flüchtlinge - Ungarns Abschied vom Asylrecht, 08.03.2017, https://www.proasyl.de/news/inhaftierung-aller-fluechtlingeungarns-abschied-vom-asylrecht/ (Entnahme: 02.11.2018).

20 Unter dem „Volk der Magyaren“ wird gemeinhin eine „homogene“ ethnische Gruppe verstanden, nicht etwa eine rechtliche Zuschreibung wie die ungarische Staatsbürgerschaft. Gegenüber dieser ethnischen Gruppe erfahren in Ungarn Minderheiten wir z.B. Roma oder Juden erhebliche Diskriminierung und Enrechtung. Siehe zum Ganzen: Marsovszky, Verfolger und Verfolgte. Antiziganismus in Ungarn, Rosa-Luxemburg-Stiftung Sachsen (Hrsg.), Leipzig, 2015; Marcks/Koob/Marsovszky, Mit Pfeil, Kreuz und Krone, Nationalismus und autoritäre Krisenbewältigung in Ungarn, 2013.

21 Vgl. anstatt vieler nur die Rede von Viktor Orbán vor dem europäischen Parlament am 26.04.2017, abrufbar: http://www.miniszterelnok.hu/rede-von-viktororban-in-der-plenarsitzung-des-europaischen-parlaments/ (Entnahme: 02.11. 2018) und Roser, Flüchtlingsfurcht ohne Flüchtlinge, ZEIT ONLINE, 18.03. 2017, abrufbar: http://www.zeit.de/politik/ausland/2017-03/ungarn-grenze-flue chtlinge-viktor-orban-internierung (Entnahme: 02.11.2018).

22 Vgl. Die Ausführungen vom Helsinki Committee Hungary, Changes in the Hungarian asylum system since 2015, abrufbar: https://www.helsinki.hu/wp- 


\section{Deutschland/Österreich}

Bevor Mitte 2015 die deutsche Bundeskanzlerin Angela Merkel entschied, dass die Bundesrepublik von ihrem in Art. 17 Dublin-III-VO gesetzlich vorgesehenen Selbsteintrittsrecht Gebrauch machen sollte und somit den Weg nach Deutschland für die vorwiegend aus Ungarn anreisenden Geflüchteten rechtlich freimachte, ${ }^{23}$ überquerten bereits über den gesamten Sommer 2015 hinweg immer mehr Geflüchtete die deutsch-österreichische Grenze. Dies veranlasste die deutschen und österreichischen Behörden, wieder verstärkte Grenzkontrollen einzuführen. Die deutschen Grenzbeamt*innen wurden in Absprache mit Österreich angewiesen, kurz vor, aber auch jenseits der deutschen Grenze, also auf österreichischem Hoheitsgebiet, Personenkontrollen durchzuführen und den Zutritt $\mathrm{zu}$ deutschem Staatsgebiet ggf. zu verwehren. Politisch begründet wurde dieses ungewöhnliche Vorgehen damit, es gebe eine sog. Transitzone zwischen dem deutschen und dem österreichischen Staatsgebiet, in dem u.a. das Recht auf Stellung eines Asylgesuchs nicht gelte. Hierbei wurde die sog. Transitzone als rechtlich-fiktiver räumlicher Bereich verstanden, es handelte sich hier nicht um eine erbaute Transitanlage wie im ungarischen Fall (s.o.). Aus diesem Grund ist auch diese Transitzone als funktionale Grenze zu verstehen. Diese Position wurde zudem vereinzelt auch von Rechtsgelehrten verteidigt. $^{24}$

content/uploads/Key-asylum-related-HHC-publications-2015-2018.pdf

(Entnahme: 06.11.2018).

23 Die Rechtmäßigkeit dieses Vorgehens seitens Deutschlands bestätigte der EuGH in seinem Urteil vom 26.07.2017, Az.: C-646/16, Rn. 100.

24 Peukert/Hillgruber/Foerste/Putzke, Nochmals: Die Politik offener Grenzen ist nicht rechtskonform, VerfBlog, 2016/3/02, http:/verfassungsblog.de/nochmalsdie-politik-offener-grenzen-ist-nicht-rechtskonform/ (Entnahme: 02.11.2018). Dabei soll hier nicht auf die intensiv geführte Diskussion eingegangen werden, welche Bestimmung der Dublin-III-VO für die Zuständigkeitszuteilung genau einschlägig ist. Dies ändert nämlich auch nichts an der Feststellung, dass Peukert u.a. jedenfalls von der Existenz eines sog. Transitbereichs zwischen der deutschen und der österreichischen Grenze ausgehen; ist doch die Frage der Zuständigkeit eines Staates nach der Dublin-III-VO der Frage nach dem Grenzverlauf nachgelagert. 
Die eigentlich nur ausnahmsweise, vorübergehend und für maximal zwei Jahre zulässigen Grenzkontrollen an der Binnengrenze zu Österreich (vgl. Art. 25, 29 Schengener Grenzkodex) wurden im Oktober 2018 durch das Bundesinnenministerium nochmals bis zum 11.05.2019 verlängert. $^{25}$ Zudem wurde ein Verwaltungsabkommen mit Griechenland abgeschlossen, das die Möglichkeit der Rückführung einer Geflüchteten Person nach Griechenland vorsieht, sofern ein EURODAC-Treffer der Kategorie 1 festgestellt wurde (die geflüchtete Person also ihren Fingerabdruck bei den griechischen Behörden abgegeben hat), eine Einreise-Verweigerung auf deutscher Seite an der deutsch-österreichischen Grenze erfolgt ist und die griechischen Behörden der Rücknahme der Person nicht binnen sechs Stunden widerspricht. ${ }^{26}$ Ein Asylgesuch können die betroffenen Personen dann in Deutschland nicht mehr stellen, auch eine Prüfung der Zuständigkeit zur Entgegennahme von Asylanträgen nach der Dublin-III-VO wird dann von Deutschland nicht mehr vorgenommen. ${ }^{27}$ Vorausgegangen war diesen Entscheidungen ein lang andauernder Streit über die Behandlung von in Deutschland ankommenden Geflüchteten zwischen Bundeskanzlerin Angela Merkel und Bundesinnenminister Horst Seehofer im Frühjahr und Sommer $2018 .^{28}$

25 Pressemitteilung des BMI vom 12.10.2018, abrufbar: https://www.bmi.bund. de/SharedDocs/pressemitteilungen/DE/2018/10/grenzkontrollen-zu-oesterreich. html (Entnahme: 06.11.2018); zur Rechtswidrigkeit der Grenzkontrollen insgesamt s. Hruschka, Gewolltes Recht, Verfassungsblog 02.11.2018, https://verfassungsblog.de/gewolltes-recht/ (Entnahme: 06.11.2018); ders., Kontrolle oder Chaos? Zur Rechtswidrigkeit der Zurückweisung von Schutzsuchenden an den europäischen Binnengrenzen, Flüchtlingsforschungsblog 18.06.2018, https://fluechtlingsforschung.net/kontrolle-oder-chaos-zur-rechtswidrigkeit-derzuruckweisung-von-schutzsuchenden-an-den-europaischen-binnengrenzen/ (Entnahme: 06.11.2018).

26 Die englische Version des Abkommens ist hier einsehbar: http://rsa egean.org/the-administrative-arrangement-between-greece-and-germany/ (Entna hme: 06.11.2018); s. außerdem Hruschka, Gewolltes Recht, Verfassungsblog 02.11.2018, https://verfassungsblog.de/gewolltes-recht/ (Entnahme: 06.11.2018).

27 Ebd.

28 Eine chronologische Dokumentation des Streits findet sich auf ZDF.de: Chronik eines Streits, Merkel, Seehofer und die Flüchtlingspolitik, ZDF.de 02.07.2018, 


\section{Rechtliche Beurteilung und rhetorische Verschiebung}

\section{Begrifflichkeit}

Rechtlich betrachtet sind alle drei oben genannten Fälle äußerst problematisch. Die Beispiele haben gemeinsam, dass jeweils davon ausgegangen wird, es gebe einen räumlichen Bereich vor oder hinter der tatsächlichen Staatsgrenze, in dem die Behörden eines Staates zwar handeln dürfen, aber rechtlich für dieses Handeln nicht verantwortlich gemacht werden können. Während die spanische Regierung von einer operativen Grenze spricht, wird in der ungarischen und teilweise der deutschen Debatte der Begriff der Transitzone verwendet. Hierbei muss aber deutlich von den regulierten Transitzonen bspw. an Flughäfen abgegrenzt werden. Im Gegensatz zu dem Transitbereich im Flughafenverfahren, der sich jedenfalls im Grundsatz durch Verrechtlichung und überprüfbare Verfahren auszeichnet, handelt es sich bei den sog. Transitzonen der deutschen Debatte vor dem Verwaltungsabkommen mit Griechenland sowie in der ungarischen Debatte um einen nicht regulierten Raum. ${ }^{29}$ Ihr lag die Annahme zugrunde, ein solcher nicht regulierter Raum bestehe natürlicherweise. ${ }^{30}$ Letztlich macht die be-

https://www.zdf.de/nachrichten/heute/chronologie-merkel-seehofer-fluechtlings politik-102.html (Entnahme 06.11.2018).

29 Siehe zum Unterschied sowie zur Kritik auch am Flughafenverfahren eingehend Kleist, Versionen fiktiver Migrationspolitik und was sie unterscheidet: Transit zentren, Flughafenverfahren und die australische non-Migration Zone, Verfas sungsblog 05.07.2018, https://verfassungsblog.de/versionen-fiktiver-migrations politik-und-was-sie-unterscheidet-transitzentren-flughafenverfahren-und-die-aus tralische-non-migration-zone/ (Entnahme: 06.11.2018); ferner Salter (Hrsg.), Po litics at the Airport, Minneapolis/London 2008.

30 Auch das BVerfG hat festgestellt, dass das Flughafenverfahren sich vom regulären Verfahren deutlich unterscheidet. Dabei betont es die Wichtigkeit von gesetzlichen Regularien für diesen Bereich, die die Gewährung eines fairen rechtsstaatlichen Verfahrens sowie Gewährung effektiven Rechtsschutzes nicht erschweren, oder vereiteln, BVerfG, Beschluss vom 14.05.1996, Az.: 2 BvR 1516/93. Siehe zur gesamten Debatte um Transitzonen auch Tohidipur, Transitzonen für Schutzsuchende - Neue Vokabel für eine alte Idee, LTO 28.10.2015, abrufbar: https://www.lto.de/recht/hintergruende/h/transitzone-fluechtlinge-asylfluechtlingskonvention-dublin-ii-non-refoulement/ (Entnahme: 02.11.2018). 
griffliche Unterscheidung zwischen operativer Grenze und Transitzone auch keinen Unterschied. Es geht in jedem Fall um die Schaffung eines rechtsfreien Raumes, der im Folgenden als funktionale Grenze bezeichnet werden soll. Die Lage an der deutsch-österreichischen Grenze ist seit dem Verwaltungsabkommen mit Griechenland zwar auf dem Papier reguliert, materiell betrachtet handelt es sich aber bei dem Bereich an der deutschen Grenze, in dem Beamt*innen Geflüchteten die Einreise verweigern dürfen sollen, ebenfalls um einen räumlichen Bereich, in dem für Geflüchtete bestimmte Rechte nicht gelten sollen. Insofern kann auch hier von einer funktionalen Grenze gesprochen werden.

\section{Funktionale Grenze im Völker- und Unionsrecht}

Völkerrechtlich kann Territorium, für das gar kein Recht gilt, nur sog. terra nullius sein, also Gebiet, das unbesiedelt ist und zu keinem Hoheitsgebiet eines Staates gehört oder unter internationaler Verwaltung steht. ${ }^{31}$ Genau das liegt aber in den hier besprochenen Beispielen nicht vor. Vielmehr bestehen die beteiligten Staaten darauf, dass der Bereich der funktionalen Grenze auch zu ihrem Hoheitsgebiet gehört. Völkerrechtlich ist dieses Konzept also nicht zu erklären.

Auch in den bi- oder multilateralen Verträgen über den konkreten Grenzverlauf ist eine funktionale Grenze im Unterschied zur rechtlich festgelegten Staatsgrenze gerade nicht vorgesehen. Schließlich widerspricht die Annahme einer funktionalen Grenze auch dem Grundkonzept des gemeinsamen europäischen Asyl- und Migrationsrechts sowie der Genfer Flüchtlingskonvention. In beiden Fällen geht es darum, effektiven Schutz vor Verfolgung und Krieg zu gewährleisten. Wie oben gezeigt, kann dies allerdings nur realisiert werden, wenn Geflüchtete die Grenze eines Staates überhaupt überqueren können und sich der faktische Grenzverlauf nicht willkürlich je nach Betrachtungsweise und politischer Entscheidung verschiebt. Zusätzlich verfolgen die Dublin-III-VO sowie weitere europarechtliche Normen ausdrücklich das Ziel, ,,refugees in orbit“ zu verhindern.

31 Zum Konzept des terra nullius siehe ICJ, Western Sahara, Advisory Opinion, 1975 ICJ 12, 38 Abs. 79 f.; kritisch zu diesem Konzept Connor, The Invention of Terra Nullius: Historical and Legal Fictions on the Foundations of Australia, 2005. 
Der Terminus ,refugees in orbit“ bezeichnet solche Fälle, in denen sich Asylsuchende zwar auf dem Gebiet der EU aufhalten, sich aber aufgrund von widersprüchlichen nationalen Rechtsnormen kein Mitgliedsstaat für die Prüfung des Asylgesuchs zuständig sieht. Insofern geht es bei dieser Doktrin der Dublin-III-VO genau genommen um eine nachgelagerte Frage der Zuständigkeit, und nicht darum, ob der Zugang zu einem schützenden System überhaupt gewährt wird. Dennoch ist insbesondere unter Berücksichtigung von Art. 3 Abs. 1 Dublin-III-VO, welcher vorsieht, dass die Mitgliedsstaaten auch solche Asylgesuche prüfen, die an ihren Grenzen gestellt werden, ein gewisser Außenbezug des Schutzzweckes der Dublin-III-VO nicht von der Hand zu weisen.

\section{Rhetorische Verschiebung}

Das Vorgehen der spanischen, ungarischen und deutschen Regierung sowie Beamt*innen wird somit zurecht als europa- und völkerrechtswidrig kritisiert. ${ }^{32}$ Dass dies aufgrund der Tatsache, dass es sich bei der Grenze zwischen Deutschland und Österreich um eine EU-Binnengrenze handelt, an-

32 ECCHR, Fallbeschreibung Melilla, S.1 f., abrufbar: https://www.ecchr.eu/ de/unsere-themen/voelkerstraftaten-und-rechtliche-verantwortung/migration-und -flucht/melilla.html?file=tl_files/Dokumente/Universelle\%20Justiz/Fallbeschrei bung_Melilla_EGMR_20171003.pdf (Entnahme: 02.11.2018); Hong, Die „Fiktion der Nichteinreise“ als Grundrechtseingriff durch normativen Tatsachenausschluss, Verfassungsblog 04.07.2018, https://verfassungsblog.de/die-fiktion-dernichteinreise-als-grundrechtseingriff-durch-normativen-tatsachenausschluss/ (En tnahme: 06.11.2018); Hruschka, Gewolltes Recht, Verfassungsblog 02.11.2018, https://verfassungsblog.de/gewolltes-recht/ (Entnahme: 06.11.2018); ders., Kontrolle oder Chaos? Zur Rechtswidrigkeit der Zurückweisung von Schutzsuchenden an den europäischen Binnengrenzen, Flüchtlingsforschungsblog 18.06.2018, https://fluechtlingsforschung.net/kontrolle-oder-chaos-zur-rechtswidrigkeit-derzuruckweisung-von-schutzsuchenden-an-den-europaischen-binnengrenzen/ (Ent nahme: 06.11.2018); Schmalz, Die Fiktion der Nichteinreise ist ein Instrument der Entrechtung, Verfassungsblog 04.07.2018, https://verfassungsblog.de/diefiktion-der-nichteinreise-ist-ein-instrument-der-entrechtung/ (Entnahme 06.11. 2018). 
ders zu bewerten sei, ${ }^{33}$ erscheint nicht plausibel. Insgesamt ist die Schaffung eines solchen faktisch rechtsfreien Raums schlicht rechtswidrig. ${ }^{34}$ Insgesamt werden hier also zur Legitimation einer rechtswidrigen Praxis an den Staatsgrenzen rhetorisch selbige verschoben.

\section{AUSWIRKUNGEN DER RHETORISCHEN VERSCHIEBUNG}

\section{Für die juristische Beschreibung der Grenze}

Es stellt sich nun die Frage, ob und wenn ja welche unmittelbaren rechtlichen Veränderungen des Grenzverlaufs sich aus den abweichenden politischen Beschreibungen ergeben. Die zehn Jahre andauernde Abschiebepraxis an den Grenzen von Melilla hat $2015 \mathrm{zu}$ einer formalen Legalisierung ebenjener auf nationaler Ebene geführt. ${ }^{35}$ Dennoch bestreitet die spanische Regierung auch weiterhin nicht den in völkerrechtlichen Verträgen festgelegten Grenzverlauf. ${ }^{36}$ Auch Ungarn hält daran fest, dass der Zaun sowie einige Meter dahinter ungarisches Staatsgebiet sind. Den eigentlichen Grenzverlauf hält die Regierung also nicht für tangiert. Schließlich stellt auch in der deutsch-österreichischen Debatte niemand den tatsächlichen Grenzverlauf auf dem Papier in Frage.

33 So aber wohl Peukert/Hillgruber/Foerste/Putzke, Nochmals: Die Politik offener Grenzen ist nicht rechtskonform, VerfBlog, 2016/3/02, https://verfassungs blog.de/nochmals-die-politik-offener-grenzen-ist-nicht-rechtskonform/ (Entnah me: 02.11.2018); vgl. auch Fn. 24.

$34 \mathrm{Zu}$ diesem Ergebnis gelangt im Fall N.D. and N.T. v. Spain auch der EGMR, Urteil vom 03.10.2017, Az.: 8675/15 und 8697/15, Rn. 52 ff.; s. auch Fn. 32.

$35 \mathrm{Zu}$ dem Phänomen der Verrechtlichung des Ausnahmezustandes siehe weiterführend Frankenberg, Ausnahmezustand, in: KJ 2017, S. 3 ff.

$36 \mathrm{Zu}$ den internationalen Verträgen vgl. EGMR, Urteil vom 03.10.2017, Az.: $8675 / 15$ und 8697/15, Rn. 53. 
Dieser Befund ist auch wichtig für die Frage, ob hier möglicherweise eine Rechtsänderung durch Gewohnheitsrecht ${ }^{37}$ oder nach dem Grundsatz des unbestrittenen Besitzstandes (uti possidetis) ${ }^{38}$ erfolgen kann. Dadurch, dass alle beteiligten Staaten offiziell verlauten lassen, an ihrer Staatsgrenze habe sich nichts geändert, wird man bereits an einer übereinstimmenden Staatenpraxis zweifeln können.

Bestätigt wird der Befund, dass sich die rechtliche Beschreibung durch eine abweichende politische Rhetorik nicht verändert, auch vom EGMR. Dessen kleine Kammer hat 2017 in zwei Urteilen die Völkerrechtswidrigkeit der spanischen Abschiebepraxis in Melilla und der Gesetzesänderungen in Ungarn festgestellt (eine Entscheidung der großen Kammer steht noch aus). ${ }^{39}$

Der EGMR attestierte Ungarn, dass die Abschiebung von Flüchtlingen auf die andere Seite des Zaunes sowie die zeitlich unbegrenzte Inhaftierung gegen Art. 3 (Verbot von unmenschlicher oder erniedrigender Behandlung), Art. 5 Abs. 1 und 4 (Recht auf Fortbewegungsfreiheit) und Art. 13 i.V.m. Art. 3 (Recht auf wirksame Beschwerde) EMRK verstößt. ${ }^{40}$ Die spanische Abschiebepraxis in Melilla verstößt laut EGMR gegen das Verbot der Kollektivausweisung (Art. 4 des 4. Zusatzprotokolls zur EMRK) sowie das Gebot eines rechtsstaatlichen Verfahrens (Art. 13 EMRK). ${ }^{41}$ Das Verbot der Kollektivausweisung soll davor schützen, dass Gruppen von Geflüchteten ohne Einzelfallprüfung abgeschoben werden, das Gebot eines rechtsstaatlichen Verfahrens sicherstellen, dass staatliche Handlungen justiziabel sind und Verantwortlichkeit geschaffen wird. Das Verbot der Kollektivausweisung ist dabei elementarer Bestandteil eines Rechtssystems zum Schutz von Menschenrechten an der Staatsgrenze während dem Gebot des

37 Zur Rechtsänderung durch Gewohnheitsrecht im Völkerrecht siehe v. Arnauld, Völkerrecht, 3. Auflage 2016, Rn. 249 ff.

38 Siehe hierzu Krajewski, Völkerrecht, 2017, § 7 Rn. 23 ff.

39 N.D. and N.T. v. Spain, EGMR, Urteil vom 03.10.2017, Az.: 8675/15 und 8697/15 (Melilla); Ilias and Ahmed v. Hungary, EGMR, Urteil vom 14.03.2017, Az.: 47287/15 (Ungarn).

40 Ilias and Ahmed v. Hungary, EGMR, Urteil vom 14.03.2017, Az.: 47287/15, Rn. $70 \mathrm{ff} ., 91 \mathrm{ff} ., 112 \mathrm{ff}$.

41 N.D. and N.T. v. Spain, EGMR, Urteil vom 03.10.2017, Az.: 8675/15 und 8697/15, Rn. 102 ff., 116 ff. 
rechtsstaatlichen Verfahrens fundamentale Bedeutung für Rechtsstaatlichkeit überhaupt zukommt. Gegen beide Grundsätze verstößt laut EGMR die spanische Abschiebepraxis in Melilla. Die Kammer betont dabei, dass es auf die Frage, ob die Grenze tatsächlich überschritten wurde, dann nicht ankommt, wenn sich die Geflüchteten auf dem Gebiet befinden, über das die spanischen Behörden ausschließliche Hoheitsbefugnisse haben bzw. ausüben. $^{42}$ Dort wo Staatsgewalt effektiv ausgeübt wird, müssen auch Rechte für Geflüchtete gelten. ${ }^{43}$ Insgesamt erfolgt also eine Änderung der Lage de jure nicht.

\section{Für das exekutive Handeln}

Während sich die rechtliche Lage auf dem Papier nicht ändert, hat die abweichende politische Beschreibung große Auswirkungen auf das tatsächliche exekutive Handeln an der Grenze. Im Fall von Melilla wurde bereits im Vorfeld durch die behördeninterne Anweisung systematisch ein Rechtsbruch vorgegeben und über zehn Jahre lang rhetorisch legitimiert. Auch das Gesetz, welches die Abschiebepraxis 2015 auf eine gesetzliche Grundlage gestellt hat, wird von der spanischen Regierung damit gerechtfertigt, es gebe zusätzlich zur tatsächlichen eine sog. operative Grenze. Der gleiche Mechanismus lässt sich in der Diskussion um das ungarische Vorgehen und die deutsch-österreichische Grenze beobachten. In beiden Fällen wurde sowohl im Vorfeld als auch nachträglich der Rechtsbruch zunächst politischrhetorisch und später gesetzlich bzw. durch ein Verwaltungsabkommen legitimiert. Dies ändert allerdings nichts an der Rechtswidrigkeit des Vorgehens (s.o.).

Ironischerweise wird häufig die Figur des Ausnahmezustandes herangezogen, um die Aussetzung von geltendem Recht zu rechtfertigen. ${ }^{44}$ Dass es sich bei diesem Recht zumeist um solches handelt, das explizit für Krisensituationen im Sinne von außergewöhnlichen Umständen geschaffen wurde, wird dabei oftmals verschwiegen. Zu solchen, die Wiedereinfüh-

42 Ebd., Rn. 52 ff.

43 Ebd., auch in Fortführung der Hirsi-Rechtsprechung (EGMR, Urteil vom 23.02.2012, Az. 27765/09).

44 Siehe hierzu umfassend Röhner, Sicherheit als rechtswissenschaftliche Praxis, in: KritV 98 (2015), S. 153 ff.; 
rung von vorübergehenden Kontrollen an den Binnengrenzen rechtfertigenden außergewöhnlichen Umständen gehören Migration und das Überschreiten der Außengrenzen durch eine große Anzahl von Drittstaatsangehörigen an sich allerdings nicht. ${ }^{45}$ Insgesamt ist anzunehmen, dass die handelnden Behörden durch die nachträgliche politische (wie formale) Rechtfertigung ihres rechtswidrigen Handelns dazu verleitet werden, sich auch in Zukunft rechtsbrüchig zu verhalten, da sie keine unmittelbaren Konsequenzen zu befürchten haben. ${ }^{46}$

\section{Für den öffentlichen Diskurs}

Schließlich ist die Auswirkung solcher Aussagen für den öffentlichen Diskurs nicht zu unterschätzen. Einerseits macht die Verrohung des öffentlichen Diskurses über geflüchtete Personen Rechtsbrüche dieser Art erst möglich. Gleichzeitig führen institutionalisierte Rechtsbrüche auch dazu, dass der öffentliche Diskurs weiter eskaliert. Es handelt sich also um eine gefährliche Legitimationsspirale, welche im schlimmsten Fall dazu führt, dass Maßnahmen ergriffen werden, die nach Sach- und Rechtslage nicht geboten wären.

45 Erwägungsgründe 1 und 5 zur „Verordnung zur Änderung der Verordnung (EG) Nr. 562/2006 zwecks Festlegung einer gemeinsamen Regelung für die vorübergehende Wiedereinführung von Kontrollen an den Binnengrenzen unter auBergewöhnlichen Umständen“: „In einem Raum ohne Kontrollen an den Binnengrenzen bedarf es einer gemeinsamen Antwort auf Situationen, die eine ernsthafte Bedrohung für die öffentliche Ordnung oder die innere Sicherheit dieses Raums, Teilen dieses Raums oder eines oder mehrerer ihrer Mitgliedstaaten darstellen, indem die vorübergehende Wiedereinführung von Kon-trollen an den Binnengrenzen unter außergewöhnlichen Umständen gestattet wird, ohne dass der Grundsatz des freien Personenverkehrs berührt wird.[...] Migration und das Überschreiten der Außengrenzen durch eine große Anzahl von Drittstaatsangehörigen sollte nicht an sich als Gefahr für die öffentliche Ordnung oder die innere Sicherheit betrachtet werden."

46 Die Frage nach mittelbaren Konsequenzen wie rechtsstaatlichen Verfahren vor supranationalen Gerichten beispielsweise gegen Ungarn vor dem EuGH ist indes eine andere. Hier soll es um die unmittelbaren Auswirkungen für die ausführenden Behörden gehen. 


\section{Konsequenz: Bedeutungsverlust der rechtlichen Beschreibung}

Zusammenfassend lässt sich festhalten, dass sich die rechtliche Lage aufgrund der oben beschriebenen Mechanismen nicht unmittelbar ändert. Sie erhält zwar Bestätigung durch judikative Entscheidungen - sowohl das Urteil des EGMR gegen Ungarn als auch das gegen Spanien beweisen, dass gewisse Grundwerte juristisch nicht verhandelbar sein dürfen - verliert aber dennoch erheblich an Bedeutung.

Dies wird einerseits hinsichtlich der mangelnden Vollstreckbarkeit von völkerrechtlichen Gerichtsurteilen gegenüber Staaten deutlich. Solche leben nämlich letztlich von der Akzeptanz der beteiligten Parteien. Zwar werden den jeweiligen Staaten signifikante Rechtsbrüche attestiert, allerdings ist zumindest in Ungarn bereits $\mathrm{zu}$ beobachten, dass die Bereitschaft, die Rechtspraxis zu ändern, gering ist. ${ }^{47}$ Insbesondere bei dem Verfahren gegen Spanien bleibt daher abzuwarten, inwieweit sich die gesetzliche Lage sowie die Abschiebepraxis am Zaun Melillas ändern werden. Entscheidungen des EGMR sind zwar auch von allen anderen Konventionsstaaten zu beachten, allerdings ist das Verfahren auf Schadensersatz ausgerichtet und bewirkt keine unmittelbare Rechtsänderung: Das jüngste Urteil mag zwar Symbolkraft haben, tatsächlich kostet es den spanischen Staat nun aber lediglich $10.000 €{ }^{48}$ Schließlich zeigen auch die Anwendung des Verwaltungsabkommens mit Griechenland sowie die nun schon über drei Jahre andauernden Binnengrenzkontrollen an der deutsch-österreichischen Grenze, dass eine Rückkehr zum aktuell geltenden Recht nicht absehbar ist. ${ }^{49}$

47 Im Gegenteil: Auch in 2018 hat Ungarn die Rechte von Geflüchteten weiter massiv eingeschränkt und kollektive Pushbacks legalisiert, siehe für eine ausführliche Übersicht der Einschränkungen seit 2015 mit weiteren Nachweisen die nachfolgende Auflistung des Helsinki Committee Hungary, Changes in the Hungarian asylum system since 2015, https://www.helsinki.hu/wp-content /uploads/Key-asylum-related-HHC-publications-2015-2018.pdf (Entnahme: 02. 11.2018).

48 N.D. and N.T. v. Spain, EGMR, Urteil vom 03.10.2017, Az.: 8675/15 und 8697/15, Rn. 126.

49 Dabei vermutet Hruschka, dass das Verwaltungsabkommen mit Griechenland vielmehr den von deutscher Seite aus erhofften Änderungen der Dublin-III-VO 
Andererseits führt die Etablierung einer von der geltenden Rechtslage abweichenden Praxis in der Administrative und Exekutive (s.o.) dazu, dass Rechtsbrüche institutionalisiert werden. Hier findet auch in Wechselwirkung mit dem öffentlichen Diskurs ein Prozess der Gewöhnung an die „neuen Verhältnisse“ statt. Dies sowie die Verrohung des öffentlichen Diskurses, nämlich die systematische und populistische Umdeutung rechtlicher Vorgaben je nach tagespolitischem Gutdünken, führen dazu, dass der rechtliche Grenzverlauf im exekutiven Handeln an der Grenze sowie der öffentlichen (rechts-)politischen Debatte verhandelbar wird. Die juristische Beschreibung verliert mithin erheblich an Bedeutung und Geltungskraft. Zwar darf zurecht eingewendet werden, dass ein gewisses Auseinanderfallen von Recht und Realität unvermeidbar ist. Insofern ist Recht kontrafaktisch. ${ }^{50}$ Das ist kein neuer Befund, in den hier besprochenen Fällen hat die rechtliche Beschreibung des Grenzverlaufs aber eine besondere Bedeutung, da es beim Zugang zum Asylrecht um Fragen von hoher Grundrechtsrelevanz geht. Letztlich kann es für die Betroffenen im schlechtesten Fall um ihre körperliche Unversehrtheit und ihr Leben gehen.

\section{E. AUSWIRKUNGEN DES BEDEUTUNGSVERLUSTES FÜR GEFLÜCHTETE}

Der oben beschriebene Bedeutungsverlust der juristischen Beschreibung der Staatsgrenze hat verschiedene weitere Konsequenzen, insbesondere für die Geflüchteten selbst. Durch die Annahme einer funktionalen Grenze wird ein rechtsfreier Raum geschaffen. Nach Auffassung der politischen Entscheidungsträger*innen sind die Beamt*innen dort zwar befugt zu handeln, allerdings sollen umgekehrt für die Betroffenen keine Rechte gelten. Dies führt dazu, dass Geflüchteten systematisch der Zugang zum Recht

zuvorkommen und entsprechen soll. Dies ändert allerdings an der Rechtswidrigkeit nach geltender Rechtslage nichts, vgl. Hruschka, Gewolltes Recht, Verfassungsblog 02.11.2018, https://verfassungsblog.de/gewolltes-recht/ (Entnahme: 06.11.2018).

50 Siehe hierzu etwa Goldmann, Dogmatik als rationale Rekonstruktion: Versuch einer Metatheorie am Beispiel völkerrechtlicher Prinzipien, in: Der Staat 53 (2014), S. $373 \mathrm{ff}$. 
verwehrt wird. Die illegalen Abschiebungen verhindern zum einen, dass den Geflüchteten rechtliches Gehör auf der administrativen Seite gewährt wird, nämlich die Stellung eines Asylantrags. Zum anderen führt diese Praxis auch dazu, dass eine nachträgliche innerstaatliche rechtliche Überprüfung des Vorgehens der Beamt*innen nicht möglich ist.

Dabei ist die Frage, ob es sich um eine illegale Abschiebung oder einen illegalen Push-Back handelt, eine Frage der Perspektive. Aus Sicht der Geflüchteten, welche die Staatsgrenze im rechtlichen Sinne überschritten haben, werden sie nach hier vertretener Ansicht abgeschoben. Aus Sicht der handelnden Behörden hat eine Einreise im rechtlichen Sinne noch nicht stattgefunden, sodass es sich nicht um eine Abschiebung, sondern nur einen Push-Back handeln kann. Letztlich ist dies aber nur eine Frage der Terminologie. Illegal bleibt das Vorgehen in jedem Fall. So hat der EGMR in seinen beiden Urteilen gegenüber Ungarn und Spanien eindrucksvoll Verstöße gegen Art. 3 (Verbot von unmenschlicher oder erniedrigender Behandlung), Art. 5 Abs. 1 (Recht auf Fortbewegungsfreiheit) und Art. 13 i.V.m. Art. 3 (Recht auf wirksame Beschwerde) EMRK (Ungarn) ${ }^{51}$ sowie Verstöße gegen Art. 4 des 4. Zusatzprotokolls zur EMRK sowie Art. 13 EMRK (das Gebot eines rechtsstaatlichen Verfahrens) ${ }^{52}$ festgestellt (s.o.). Wichtigste Feststellung war hierbei, dass dort wo Staatsgewalt effektiv ausgeübt wird, auch Rechte für Geflüchtete gelten müssten. ${ }^{53}$ Dies müsste in der Form auch an der österreichisch-deutschen Grenze gelten. Hier wird allerdings Geflüchteten ihr subjektives Recht auf Prüfung des zuständigen Mitgliedsstaates ${ }^{54}$ sowie das Recht zur Asylantragstellung rechtswidrig genommen (s.o.).

51 Ilias and Ahmed v. Hungary, EGMR, Urteil vom 14.03.2017, Az.: 47287/15, Rn. $70 \mathrm{ff} ., 91 \mathrm{ff} ., 112 \mathrm{ff}$.

52 N.D. and N.T. v. Spain, EGMR, Urteil vom 03.10.2017, Az.: 8675/15 und 8697/15, Rn. 102 ff., 116 ff.

53 Ebd., auch in Fortführung der Hirsi-Rechtsprechung (EGMR, Urteil vom 23.02.2012, Az. 27765/09).

$54 \mathrm{Zu}$ dem Befund, dass es sich bei den entsprechenden Normen aus der Dublin-IIIVO um subjektive Rechte handelt s. EuGH, Urteile vom 07.06.2016, Az.: C$63 / 15$ und $\mathrm{C}-115 / 15$. 
Es kann letztlich nicht oft und eindringlich genug wiederholt werden: Der Bedeutungsverlust der rechtlichen Beschreibung der Staatsgrenze führt dazu, dass sich schutzsuchende Menschen faktisch in einem rechtsfreien Raum befinden.

\section{F. FAZIT}

Dieser Beitrag hat gezeigt, dass Schutzsuchende an den EU-Außen- wie Innengrenzen zunehmend in faktisch rechtsfreie Räume gedrängt werden. Diese rechtsfreien Räume entstehen dabei primär durch die Rhetorik und Handlungen politischer Entscheidungsträger*innen. Sekundär wurden einige dieser rechtsfreien Räume in der Zwischenzeit formal legalisiert oder mit einer gesetzlichen Grundlage versehen. Die Signifikanz dieses Problems ergibt sich daraus, dass es sich bei den hier behandelten Fällen um solche im hochsensiblen Grundrechtsbereich der Betroffenen handelt. Dies unterscheidet sie auch von sonstigem rechtsbrüchigem Verhalten seitens Behörden: Zwar mag die willentlich unrechtmäßige Verweigerung einer Baugenehmigung für die Betroffenen äußerst ärgerlich sein, um Überleben von Krieg und staatlicher Verfolgung geht es dort jedoch nicht.

Dass es nach den Erfahrungen des Zweiten Weltkrieges offenbar wieder öffentlich vertretbar ist, Geflüchteten systematisch den Zugang zu einem schützenden System zu verwehren, ist in letzter Konsequenz nicht nur schockierend, sondern lässt die aufmerksamen Beobachter*innen auch ernsthaft an dem historischen Gedächtnis der handelnden Personen sowie der stummen Öffentlichkeit zweifeln.

In diesem Sinne muss die rechtliche sowie gesellschaftliche Öffentlichkeit der rhetorischen Verschiebung von Staatsgrenzen und der damit verbundenen Schaffung eines faktisch rechtsleeren Raums vehement widersprechen. Sie muss sich auf die humanitären Grundwerte des modernen und aufgeklärten Zusammenlebens besinnen und rechtsbrüchigem Verhalten und dem daraus folgenden Bedeutungsverlust von auf Schutzgewährung ausgelegtem Recht lautstark entgegentreten. 


\section{LITERATUR}

European Center for Constitutional Rights (Hrsg.), Fallbeschreibung: Die spanisch-marokkanische Grenze bei Melilla - Push-Backs im rechtsfreien Raum, abrufbar: https://www.ecchr.eu/fileadmin/Fallbeschreib ungen/Fallbeschreibung_Melilla_ECtHR_ECCHR_DE.pdf (Entnahme 02.11.2018).

Frankenberg, Günter, Ausnahmezustand, in: KJ 2017, S. 3 ff.

Goldmann, Matthias, Dogmatik als rationale Rekonstruktion: Versuch einer Metatheorie am Beispiel völkerrechtlicher Prinzipien, in: Der Staat 53 (2014), S. 373 ff.

Helsinki Committee Hungary (Hrsg.), Pushed Back at the Door: Denial of Access to Asylum in Eastern EU Member States, 2017, S. 12 ff., abrufbar: https://www.proasyl.de/wp-content/uploads/2015/12/pushed_back. pdf (Entnahme: 02.11.2018).

Dies. (Hrsg.), Changes in the Hungarian asylum system since 2015, abrufbar: https://www.helsinki.hu/wp-content/uploads/Key-asylum-relatedHHC-publications-2015-2018.pdf (Entnahme: 02.11.2018).

Hong, Mathias, Die „Fiktion der Nichteinreise“ als Grundrechtseingriff durch normativen Tatsachenausschluss, Verfassungsblog 04.07.2018, https://verfassungsblog.de/die-fiktion-der-nichteinreise-als-grundrechts eingriff-durch-normativen-tatsachenausschluss/ (Entnahme: 06.11.2018).

Hruschka, Constantin, Gewolltes Recht, Verfassungsblog 02.11.2018, https://verfassungsblog.de/gewolltes-recht/ (Entnahme: 06.11.2018).

Ders., Kontrolle oder Chaos? Zur Rechtswidrigkeit der Zurückweisung von Schutzsuchenden an den europäischen Binnengrenzen, Flüchtlingsforschungsblog 18.06.2018, https://fluechtlingsforschung.net/kontrolleoder-chaos-zur-rechtswidrigkeit-der-zuruckweisung-von-schutzsuchen den-an-den-europaischen-binnengrenzen/ (Entnahme: 06.11.2018).

Kleinschmidt, Christoph, Semantik der Grenze, in: APuZ 4-5/2014, S. 3.

Kleist, Olaf J., Versionen fiktiver Migrationspolitik und was sie unterscheidet: Transitzentren, Flughafenverfahren und die australische nonMigration Zone, Verfassungsblog 05.07.2018, https://verfassungs blog.de/versionen-fiktiver-migrationspolitik-und-was-sie-unterscheidettransitzentren-flughafenverfahren-und-die-australische-non-migrationzone/ (Entnahme: 06.11.2018) 
Leibholz, Gerhard, Das Verbot der Willkür und des Ermessensmißbrauches im völkerrechtlichen Verkehr der Staaten, in: ZaöRV 1929, S. 77 ff.

Marsovszky, Magdalena, Verfolger und Verfolgte. Antiziganismus in Ungarn, Rosa-Luxemburg-Stiftung Sachsen (Hrsg.), Leipzig, 2015.

Marcks, Holger/Koob, Andreas/Marsovszk, Magdalenay, Mit Pfeil, Kreuz und Krone, Nationalismus und autoritäre Krisenbewältigung in Ungarn, 2013.

PRO ASYL (Hrsg.), Inhaftierung aller Flüchtlinge - Ungarns Abschied vom Asylrecht, 08.03.2017, https://www.proasyl.de/news/inhaftierung-allerfluechtlinge-ungarns-abschied-vom-asylrecht/ (Entnahme: 02.11.2018).

Röhner, Cara, Sicherheit als rechtswissenschaftliche Praxis, in: KritV 98 (2015), S. $153 \mathrm{ff}$.

Salter, Mark B. (Hrsg.), Politics at the Airport, Minneapolis/London 2008

Schmalz, Dana, Die Fiktion der Nichteinreise ist ein Instrument der Entrechtung, Verfassungsblog 04.07.2018, https://verfassungsblog.de/diefiktion-der-nichteinreise-ist-ein-instrument-der-entrechtung/ (Entnahme 06.11.2018).

Tohidipur, Timo, Transitzonen für Schutzsuchende - Neue Vokabel für eine alte Idee, LTO 28.10.2015, abrufbar: https://www.lto.de/recht/hin tergruende/h/transitzone-fluechtlinge-asyl-fluechtlingskonvention-dub lin-ii-non-refoulement/ 


\title{
Zum Spannungsfeld zwischen Liberalismus und Migrationspolitik
}

\author{
Kentaro Inagaki
}

\section{EINLEITUNG}

Der deutsche Politologe Claus Leggewie legte in einem Artikel der Frankfurter Rundschau ${ }^{1}$ ein Denkexperiment vor. Es ging dabei darum, ,den Flüchtlingen aus den Jahren 2014/15 sofort und ungeachtet ihrer Herkunft ein Bleiberecht zu gewähren“" (Leggewie, 2015), wobei auch diejenigen eingeschlossen werden sollten, ,die sich als ,unregistrierte“ Personen schon länger illegal auf dem Gebiet der Bundesrepublik Deutschland aufhalten“ (Leggewie, 2015). Zudem sollten die Personen, ,solange [Ihnen] die Gewährung nationaler Staatsangehörigkeiten auf Grund fehlender Voraussetzungen verwehrt bleibt", die europäische Staatsangehörigkeit erhalten (Leggewie, 2015).

Leggewie verdeutlicht in seinem 2017 publizierten Buch mit dem Titel Europa zuerst! $!^{2}$ die Bedeutsamkeit des Wechsels von der Nationalität zur Bürgerschaft. In diesem Buch wiederholt Leggewie die Forderung nach der

1 Claus Leggewie, „Unionsbürgerschaft für Flüchtlinge: EU-Pass als Ausweg aus der Krise“, in: Frankfurter Rundschau. 22. 10. 2015 (http://www.fr.de/politik/ unions-buergerschaft-fuer-fluechtlinge-eu-pass-als-ausweg-aus-der-krise-a-431 867: Letzter Zugriff: 18. 09 2017).

2 Claus Leggewie, Europa zuerst! Eine Unabhängigkeitserklärung. Berlin: Ullstein Buchverlag, 2017. 
„Verleihung der Unionsbürgerschaft an staatslose Flüchtlinge“ (Leggewie, 2017, 135). Hinter seinem Vorschlag steht die Verschiebung von „Kriterien für Mitgliedschaft und Zugehörigkeit“" (Leggewie, 2017, 135) in der Europäischen Union (EU): Der Wahrnehmung Leggewies nach, sei der Fokus dieser Kriterien „,von »Nationalität« in Richtung »Bürgerschaft«" (Leggewie, 2017, 135) verlagert.

Leggewie nimmt selbst den utopischen Ton seines Vorschlages an (Leggewie, 2015; ders, 2017, 135). Seinen Vorschlag kann man im Anschluss an die These, die Christian Joppke in seinem Buch Citizenship and Immigration $(2010)^{3}$ vorlegelegt hat, analysieren. Darin schildert Joppke, wie die Migrationspolitik im Laufe der Zeit liberaler wird. Anhand der Beispiele nicht nur von klassischen Migrationsstaaten wie den USA, sondern auch von westeuropäischen Ländern wie Deutschland, behauptet er, dass die Politik der Bürgerschaft inklusiv geworden sei (Joppke, 2010). ${ }^{4}$ Problematisch in der Analyse Joppkes ist aber, so sagt Kashiwazaki, dass er die Kontrolle der Staatsgrenzen fast außer Acht lässt (Kashiwazaki, 2016, 48), die heutzutage immer wieder an Bedeutung gewinnt.

Im Zusammenhang mit der These von Joppke stellt Kashiwazaki sich die Frage: Wie ist die Exklusion zu verstehen, die durch den Liberalismus selbst hervorgebracht wird? ${ }^{5}$ Diese Frage scheint auch mit dem Vorschlag Leggewies mehr oder weniger zu tun zu haben. Es soll zunächst gesagt werden, dass ,der Liberalismus die Migrationspolitik eines Staates inklusiv und gleichzeitig auch exklusiv machen kann“ (Kashiwazaki, 2016, 49, Betonung von Kashiwazaki).

Leggewie erwähnt selbst das Moment der Entstehung von Inklusion und Exklusion in Staatsgrenzen (Leggewie, 2017, 131). Aber er ist der

3 Christian Joppke, Citizenship and Immigration. Cambridge: Polity Press, 2010.

4 Diese Zusammenfassung der Diskussion von Joppke basiert auf dem folgenden Aufsatz von Kashiwazaki. Kashiwazaki Masanori (2016), „Imin seisaku ni okeru liberalism no niritsuhaihan - tōitsu Doitsu no jirei“ („The Antinomy of Liberalism in German Migration Policy“), in: Tokyo Gaikokugo Daigaku Ronshū (Area and Culture Studies). 92: 45-63 (auf Japanisch), hier, 47.

5 Ibid., 48. Kashiwazaki erwähnt dabei den Aufsatz von Orgad. Liav Orgad (2010), (,Illiberal Liberalism: Cultural Restrictions on Migrations and Access to Citizenship in Europe“, in: The American Journal of Comparative Law. 58 (1): 53-105. 
Meinung, dass in modernen Gesellschaften Exklusionen „faktisch nur noch temporäre Ausschlüsse“ (Leggewie, 2017, 131) seien. Dabei spielt seiner Meinung nach die Fremdheit keine große Rolle. Es bleibt aber fragwürdig, ob man die Fremdheit nur als ,ein allgemeines Merkmal moderner Gesellschaften“ (Leggewie, 2017, 132) ${ }^{6}$ bezeichnen kann. Wenn man den Begriff „Grenze“ in Betracht zieht, sieht die Fremdheit wahrscheinlich anders aus. Im vorliegenden Beitrag soll zunächst das Spannungsfeld von Nation und Ausländern in der Migrationspolitik zur Diskussion gestellt werden. Daran anschließend wird der Vorschlag von Leggewie in Bezug auf den Begriff „Grenze“ gelesen. Schließlich soll auf das Paradox der „liberalen“ Diskurse aufmerksam gemacht werden.

\section{SPANNUNG VON NATION UND AUSLÄNDERN}

Historisch betrachtet hat sich die deutsche Migrationspolitik wie folgt verändert: Bald nach dem Ende des Zweiten Weltkrieges hat die Bundesrepublik Deutschland Ausländer aus anderen Staaten wie Italien, Spanien und der Türkei als „Gastarbeiter“ aufgenommen, um die eigene Wirtschaftskraft zu verbessern. Seit den 1970er Jahren wurde die wirtschaftliche Migration allmählich reguliert. ${ }^{8}$

Sato (2017) ${ }^{9}$ weist auf die Bedeutsamkeit des Ausländergesetzes der Bundesrepublik Deutschland hin, das im Jahr 1965 verabschiedet wurde

6 Leggewie sagt gleichzeitig auch, dass Fremdheit „kein Exklusionsgrund“ (Leggewie, 2017, 132) ist.

7 Man muss aber die wichtige Anmerkung von Leggewie betrachten. Leggewie, 2017, 137-138. „Zwar bleiben klare, auch wieder exklusive Grenzen nach außen gezogen, die aber keinen Festungscharakter annehmen müssen, sondern offen sind für humanitäre Aktionen und für die Inklusion von Flüchtlingen - eben als Anwärter auf eine Unionsbürgerschaft".

8 Kobayashi Kaoru (2009), „Doitsu no imin seisaku ni okeru ,tōgō no shippai““ („Germany's ,Failed Integration“ Immigration Policy“), in: Europe Kenkyū (European Studies). 8: 119-139 (auf Japanisch), hier, 120-121.

9 Sato Shigeki (2017), „Kokumin kokka to gaikokujin no kenri - sengo Doitsu no gaikokujin seisaku kara“ („Nation-State and the Rights of Foreigners: The Case of the Foreigners Policy in Postwar Germany“, in: Shakai Shirin (Hosei Journal 
(Sato, 2017, 68). Im zweiten Artikel dieses Ausländergesetzes liest man die folgende Bestimmung:

»(1) Ausländer, die in den Geltungsbereich dieses Gesetzes einreisen und sich darin aufhalten wollen, bedürfen einer Aufenthaltserlaubnis. Die Aufenthaltserlaubnis darf erteilt werden, wenn die Anwesenheit des Ausländers Belange der Bundesrepublik Deutschland nicht beeinträchtigt. $\ll^{10}$

Aber wenn ein Ausländer „Belange der Bundesrepublik“ beeinträchtigt hätte, könnte er theoretisch ausgewiesen werden. Und man findet tatsächlich die Bestimmung der Ausweisung im zehnten Artikel des Ausländergesetzes von 1965:

»(1) Ein Ausländer kann ausgewiesen werden, wenn 1. er die freiheitliche demokratische Grundordnung oder die Sicherheit der Bundesrepublik Deutschland gefährdet. $[\ldots] \ll^{11}$

Darin sieht man den Versuch, das Grundkonzept der Bundesrepublik, nämlich „die freiheitliche demokratische Grundordnung“ zu sichern. Damit sollten die Interessen des Deutschen, dessen Definition sich im ersten Absatz Art. $116 \mathrm{GG}^{12}$ befindet, gewährleistet werden.

of Sociology and Social Sciences). 63 (4): 59-97 (auf Japanisch). Die folgende Beschreibung über das Spannungsfeld von Nation und Ausländern in Bezug auf die Rechte basiert auf diesem Aufsatz von Sato. Ziel dieses Aufsatzes von Sato besteht darin, zu schildern, wie auch die Ausländer die Rechte, die ursprünglich nur die Staatsangehörige haben, im Laufe der Zeit bekommen. Sato untersucht außerdem das Dilemma im Bereich der Migrationspolitik des liberalen Nationalstaates.

10 Ausländergesetz vom 28. April 1965, in: Bundesgesetzblatt, Teil I, Nr. 19. (Bonn, am 8. Mai 1965), § 2. 1, 353a.

11 Ausländergesetz vom 28. April 1965, § 10. 1, 355a. Zu diesen zwei Artikeln des Ausländergesetzes von 1965, s. Sato, 2017, 68.

12 Grundgesetz, § 116. ,(1) Deutscher im Sinne dieses Grundgesetzes ist vorbehaltlich anderweitiger gesetzlicher Regelung, wer die deutsche Staatsangehörigkeit besitzt oder als Flüchtling oder Vertriebener deutscher Volkszugehörigkeit oder als dessen Ehegatte oder Abkömmling in dem Gebiete des Deutschen Rei- 
Sato weist auch auf die strukturelle Unterscheidung zwischen den Deutschen und den Ausländern im Grundgesetz hin (Sato, 2017, 68; 70). Wendet man sich dem ersten Teil des Grundgesetzes zu, der die Grundrechte umfasst, fällt auf, dass einige Grundrechte wie Gleichheit vor dem Gesetz (Art. 3 Abs. 1), Glaubensfreiheit (Art. 4 Abs. 1) und Meinungsfreiheit (Art. 5 Abs. 1) von jedermann in Anspruch genommen werden können (Sato, 2017, 70-71). Aber es gibt auch Grundrechte, die nur Deutschen zuerkannt werden. Dazu gehören z.B. Versammlungsfreiheit (Art. 8 Abs. 1), Vereinigungsfreiheit (Art. 9 Abs. 1), Freizügigkeit im ganzen Bundesgebiet (Art. 11 Abs. 1) usw. (Sato, 2017, 71).

Daraus weist Sato darauf hin, dass es zwei verschiedene Kategorien der Grundrechte gibt: Einerseits findet man diejenigen, die nur die Deutschen haben. Anderseits gibt es auch die universalen Grundrechte, die sich aus der Würde des Menschen ableiten (Sato, 2017, 70).

\section{GRENZE ALS PHÄNOMEN}

Die Koexistenz von einerseits den Grundrechten für Deutsche und anderseits den allgemeinen Grundrechten ist im Grundgesetz der Bundesrepublik festgeschrieben. Versteht man unter Nationalstaat einen „Staat der Nation“, ist es kein Wunder, dass der Nationalstaat zu aller erst seine Nation zu verteidigen sucht (Sato, 2017, 60). Der Begriff citizenship, der seit dem klassischen Werk von Thomas H. Marshall mit dem Titel Citizenship and Social Class (1950) ${ }^{13}$ an Bedeutung gewinnt, verweist auf ein entscheidendes Charakteristikum des modernen Nationalstaates: Während er gegenüber seiner Nation inklusiv ist, ist er gegenüber einigen Gruppen, zu denen auch Ausländer zu zählen sind, mehr oder minder exklusiv (Sato, 2017, 60). Dies bedeutet aber nicht, so Sato, dass sich die Ausländer im Territorium eines Nationalstaates nicht aufhalten dürfen (Sato, 2017, 60). Wie im vorhergehenden Teil erwähnt wird, hat z.B. die Bundesrepublik Deutschland Aus-

ches nach dem Stande vom 31. Dezember 1937 Aufnahme gefunden hat“ (https://www.gesetze-im-internet.de/gg/art_116.html: Letzter Zugriff: 29.12. 2018). Dazu, s. Sato, 2017, 69.

13 Thomas H. Marshall, Citizenship and Social Class, and Other Essays. Cambridge: Cambridge University Press, 1950. Dazu, vgl. Sato, 2017, 60. 
länder als „Gastarbeiter“ aufgenommen. Zudem besagt Art. 16a GG, dass „politisch Verfolgte Asylrecht genießen“ (vgl. Sato, 2017, 85). ${ }^{14}$

Dennoch gibt es vor allem im Bereich der Migrationspolitik ein Dilemma, nämlich die unterschiedliche Behandlung von Nation und Ausländern. Obwohl es das allgemeine Prinzip der Menschenwürde gibt, das man im ersten Artikel des Grundgesetzes findet ${ }^{15}$, werden Angehörige der Nation und Ausländer unterschiedlich behandelt (Sato, 2017, 65-66).

Dabei soll man den Begriff „Grenze“ zur Diskussion stellen. Wie Paa$\mathrm{si}^{16}$ zeigt, sind die Grenzen als die Phänomena anzusehen, die immer je nach Kontexten vorkommen. Sie können seiner Meinung nach als „Prozes-

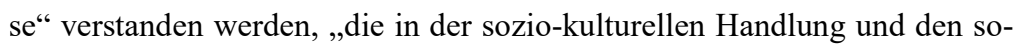
zio-kulturellen Diskursen existieren“. ${ }^{17}$

Zunächst soll der Begriff in zwei Kategorien unterschieden werden. Zum einen sind die materiellen Grenzen zur Diskussion zu stellen. Anderseits sollen die sozio-psychologischen Grenzen genannt werden. ${ }^{18}$

Die sozio-psychologischen Grenzen unterscheiden gesellschaftlich Majorität und Minderheiten, „Wir“ und „Sie“, sogar Freund und Feind (Haba, 2011, 40). Die sozio-psychologischen Grenzen machen den Unterschied

14 Grundgesetz, § 16a. „Politisch Verfolgte genießen Asylrecht“ (https://www. gesetze-im-internet.de/gg/art_16a.html: Letzter Zugriff: 29. 12. 2018). Von dem hier genannten Asylrecht werden z.B. die sog. Wirtschaftsflüchtlinge ausgeschlossen. Zu diesem Punkt, s. den Beitrag von Merve Kania in diesem Band.

15 Grundgesetz, § 1. „(1) Die Würde des Menschen ist unantastbar. Sie zu achten und zu schützen ist Verpflichtung aller staatlichen Gewalt" (https://www. gesetze-im-internet.de/gg/art_1.html: Letzter Zugriff: 29. 12. 2018).

16 Anssi Paasi (1998), „Boundaries as Social Processes: Territoriality in the World of Flows“, in: Geopolitics. 3 (1): 69-88.

17 Ibid., 72.

18 Diese Dichotomie von Grenzen basiert auf der Beschreibung der folgenden Arbeit von Haba. Haba Kumiko (2011), „Kakudai EU ni okeru kyōkaisen to citizenship - European Identity to Xenophobia (yosomonogirai) no sōkoku -“ („Border and Citizenship in the Enlarged EU: Conflict between European Identity and Xenophobia"), in: Shakai Shirin (Hosei Journal of Sociology and Social Sciences). 57 (4): 35-53 (auf Japanisch), hier, 35-36, 40-41. 
zwischen Ego und den Anderen deutlich. Dadurch, dass man das „Eigene“ bestimmt, wird anscheinend klargemacht, wer ,wir“ sind und wer nicht. ${ }^{19}$

Wenn man dann diese Konzeptionen der Grenzen auf die EU anwendet, tauchen verschiedene, aber eng zusammenhängende Fragen auf. Blickt man allein auf die materiellen Grenzen der EU, so ergibt sich die Frage nach der Ausgestaltung des Außengrenzschutzes, was seinerseits verschiedene Folgeprobleme aufwirft (z.B. das Überwachungssystem, die Identifizierung der Flüchtlinge usw.).

Trotzdem muss man auch über „die Liquidität der Grenzen“ nachdenken. ${ }^{20}$ Obwohl die territorialen, materiellen Grenzen schon „festgelegt“ zu werden scheinen, werden auch innerhalb der Gesellschaft der Mitgliedsländer die sozio-psychologischen Grenzen gezogen, welche die Rechtspopulisten in ihren politischen Diskursen betonen. Man muss dabei die „doppelte Fremdheit“ bzw. die ,,interne / externe Fremdheit" ${ }^{21}$ in Kauf nehmen.

19 Die Beschreibung in diesem Paragraph basiert auf dem Aufsatz von Haba. Haba, 2011, 40. Vgl. Paasi, 1998, 75, 80-81; Leggewie, 2017, 23.

20 Vgl. Namba Satoru (2016), „EU kokkyō chiiki ni okeru < kyōkai > no politics: Ōshū nanmin kisei regime no kōchiku to Tunisia jin nanmin“ (,Politics of « Borderlands » in the European Union: Construction of a EUropean Immigration Control Regime and Tunisian Refugees after the Arab Spring“, in: Kyōkai Kenkyü (Japan Border Review). 7: 45-70 (auf Japanisch), hier, 48-49.

$21 \mathrm{Zu}$ diesen Begriffen, s. Etienne Balibar (2010), „At the Borders of Citizenship: A Democracy in Translation?“, in: European Journal of Social Theory. 13 (3): 315-322, hier, 321; Katja F. Aas (2011), „,Crimmigrant“ Bodies and Bona Fide Travelers: Surveillance, Citizenship and Global Governance“, in: Theoretical Criminology. 15 (3): 331-346, hier, 343. Vgl. Takemura Nariyoshi (2016), „Nanmin imin tōsei to hanzai tōsei no yūgō oyobi fuhenteki jinken no saikōchiku - kyōkai no kakusan shintō, kanshi tōsei no fuhenkōjōka, kiken / anzen paradigm no datsukōchiku“ („Fusion of Refugee / Immigration Control and Crime Control, and Reconstruction of Universal Human Rights: DiffusionsPenetration of Border, Generalization of Surveillance-Control, and Deconstruction of Risk / Security Paradigm“, in: Toin Hōgaku (Toin Law Review). 23 (1): 1-29 (auf Japanisch), hier, 16. 


\section{UNIONSBÜRGERSCHAFT UND "PARADOXER" LIBERALISMUS}

In Bezug auf die sozio-psychologischen Grenzen soll man sich mit der Frage nach der europäischen Bürgerschaft beschäftigen. Wie im Vertrag von Maastricht (1993) erstmals eingeführt wurde, ist derjenige als Unionsbürger anzusehen, der ,die Staatsangehörigkeit eines Mitgliedstaats besitzt. Die Unionsbürgerschaft tritt zur nationalen Staatsbürgerschaft hinzu, ersetzt sie aber nicht". 22

Bezüglich des Vorschlags von Leggewie, mit dem der vorliegende Beitrag eingeleitet wurde, ist es wichtig, anzumerken, dass die Unionsbürgerschaft mit der Staatsangehörigkeit eines Mitgliedstaates eng verbunden ist. Dies kann man auch folgendermaßen ausdrücken: Die Unionsbürgerschaft hängt von der souveränen Entscheidung der Staaten über ihre Bürger ab. ${ }^{23}$

Es gibt zwar in der EU verschiedene Arten und Weisen, das Recht als Unionsbürger in der demokratischen Öffentlichkeit auszuüben (wie etwa European Citizens' Initiative). ${ }^{24}$ Es würde doch fragwürdig sein, ob die sozial benachteiligten Menschen, zu denen auch die Flüchtlinge gezählt werden sollen, die Unionsbürgerschaft völlig genießen können. Haba stellt sich die Frage, „ob das Netzwerk der Unionsbürgerschaft auch die sozial benachteiligten Menschen und Outsider umfassen kann“ (Haba, 2011, 43).

22 Vertrag über die Arbeitsweise der Europäischen Union. Konsolidierte Fassung. Artikel 20. (http://eur-lex.europa.eu/legal-content/DE/TXT/HTML/?uri=CELE X:12012E/TXT\&from=DE: Letzter Zugriff: 20. 09 2017). Vgl. Leggewie, 2017, 131.

23 Vgl. Tsuchiya Takeshi (2005), „EU to minshuteki citizenship - daisankoku kokumin no hōsetsu o chūshin ni - “ (,The EU and Democratic Citizenship - Focusing on the Inclusion of Third Country Nationals -“), in: Nihon EU Gakkai Nenpō (EU Studies in Japan). 25: 244-266 (auf Japanisch), hier, 245; Haba, 2011, 39-40, 43; Nakamura Kengo (2018), „EU wa ekkyō suru hito no kenri o dokomade mitometeiruka? EU shimin, imin, nanmin no genjō o fumaete“ („To What Extent Does the European Union Guarantee the Rights of Person Who Crosses Borders?: EU Citizens, Immigrants, and Refugees“, in: The Keizaigaku Zasshi (Journal of Economics). 119 (1): 41-80 (auf Japanisch), hier, 47-48.

24 Vgl. Leggewie, 2017, 137. 
Hinter dieser Frage steht die schnelle Verbreitung der Fremdenfeindlichkeit in Europa (Haba, 2011, 43).

Die Abhängigkeit der Unionsbürgerschaft von der Staatsangehörigkeit eines Mitgliedstaates der EU wird schon von Leggewie selbst erkannt (Leggewie, 2015; ders, 2017, 131; 133). Die Verleihung der europäischen Staatsangehörigkeit an die Personen, die ,,auf Grund fehlender Voraussetzungen die Gewährung nationaler Staatsangehörigkeit verwehrt bleibt“ (Leggewie, 2015), ist zwar ein Versuch, die „Kriterien für Mitgliedschaft und Zugehörigkeit von »Nationalität« in Richtung »Bürgerschaft« [zu] verschieben“ (Leggewie, 2017, 135). Aber man könnte die vermutete Funktion der Unionsbürgerschaft in Frage stellen: Wenn die Unionsbürgerschaft an die genannten Flüchtlinge verliehen werden könnte, wäre es doch vielleicht noch schwierig, einzuschätzen, wie die Unionsbürgerschaft funktionsfähig bleibt. $^{25}$

Leggewies Vorschlag kling jedenfalls ,liberal“. Der Liberalismus per se ist aber ein sehr paradoxer Begriff. Bemerkenswert ist es zunächst, dass die liberalen Diskurse einerseits zur Aufnahme der Flüchtlinge führen können, wie man beim Vorschlag von Leggewie wahrnimmt. Anderseits jedoch muss man beachten, dass die „liberalen“ Diskurse auch als die Logik der Exklusion fungieren können, wie Kashiwazaki hinweist (Kashiwazaki, 2016, 48).

Man kann im Großen und Ganzen zwei gegenseitige Reaktionen auf den Flüchtlingszustrom wahrnehmen. Eisnecker und Schupp (2016) ${ }^{26}$ sagen, dass ,,[n]ach wie vor in der Öffentlichkeit die Ansicht vor[herrscht], dass Kriegsflüchtlinge in Deutschland aufzunehmen seien“ (Eisnecker und Schupp, 2016, 158). Eisnecker und Schupp deuten zudem aber darauf hin, dass ,die Akzeptanz von anderen Gründen der Beantragung von Asyl gesunken [ist]“ (Eisnecker und Schupp, 2016, 158). Die Reaktionen hängen also von den Gründen der Flucht ab.

25 Leggewie nimmt schon die Notwendigkeit der Fortentwicklung der Unionsbürgerschaft in Kauf. Vgl. Leggewie, 2017, 134. Dazu, vgl. auch Leggewie, 2017, 136-138.

26 Philipp Eisnecker und Jürgen Schupp (2016), „Flüchtlingszuwanderung: Mehrheit der Deutschen befürchtet negative Auswirkungen auf Wirtschaft und Gesellschaft", in: DIW Wochenbericht. 8: 158-164. 
Die negativen Reaktionen auf die Flüchtlinge im politischen Diskurs werden meistens von den Rechtsextremisten sowie Rechtspopulisten vertreten. Der Beschreibung von Takemura (2016) ${ }^{27}$ nach lässt sich die Bewegung der Rechten dadurch kennzeichnen, dass sie den Unterschied der „Rassen“ betonen und die Gewalttätigkeit fördern. ${ }^{28}$

Wichtig ist aber, dass die Rechtsextremisten die Frage der Flüchtlinge einfach zu einer Frage der staatlichen Sicherheit umdeuten. ${ }^{29}$ Die „Gefährlichkeit" der Migranten, welche die Rechtsextremisten wahrnehmen, erstrecken sich auf die verschiedenen Themen wie nationale Sicherheit, Wirtschaft und Kultur (vgl. Lazaridis und Wadia, 2015, 4; Takemura, 2016, 13).

Nach den Terroranschlägen am 11. September 2001 wird zwar die Sicherheit des Staates im politischen Diskurs wichtiger (Lazaridis und Wadia, 2015, 1). Auf diesem Grund aber behaupten die Rechtspopulisten immer wieder, dass die Flüchtlinge die gesellschaftliche Sicherheit bedrohen könnten (Hogan und Haltinner, 2015; Takemura, 2016, 11). Gerade deswegen werden Flüchtlinge durch die Rechtspopulisten als der „Feind“ betrachtet. Dabei wird die falsche „Kausalität“ zwischen der Kriminalität und der Zunahme der Migranten / Flüchtlingen betont (Takemura, 2016, 2-3; 1819).

Solche nicht auf seriösen Quellen basierende Meinungen kann man auf europäischer Ebene betrachten. ${ }^{30}$ Wenn man sich die Situation der osteuro-

27 Takemura, 2016, „Fusion of Refugee / Immigration Control“ (wie Anm. 21).

28 Takemura, 2016, 11. Die Beschreibung von Takemura beruht dabei auf dem folgenden Aufsatz von Jackie Hogan und Kristin Haltinner. Jackie Hogan und Kristin Haltinner (2015), „Floods, Invaders, and Parasites: Immigration Threat Narratives and Right-Wing Populism in the USA, UK and Australia“, in: Journal of Intercultural Studies. 36 (5): 520-543.

29 Gabriella Lazaridis und Khursheed Wadia, „Introduction“, in: Gabriella Lazaridis und Khursheed Wadia (eds.), The Securitisation of Migration in the EU: Debates since 9/11. Hampshire: Palgrave Macmillan, 2015, 1-16, hier, 4. Dazu, vgl. Takemura, 2016, 13-14. Takemura führt in seinem Aufsatz diese Einleitung zum Sammelband von Lazaridis und Wadia an und fasst die Diskussion von Lazaridis und Wadia zusammen.

30 Vgl. Yamaoka Kenjiro (2018), „Nanmin kenkyū eno shisōshiteki approach liberal na nanminkan o koete“ („Beyond a Liberal View of Refugees“), in: 
päischen Länder wie Ungarn und Polen anschaut, kann man eine Politik wahrnehmen, die nur die eigenen staatlichen Interessen berücksichtigt. Der Brexit mag auch in diesem Zusammenhang verstanden werden (Yamaoka, 2018, 118). Auf jeden Fall ist es jetzt bemerkenswert, dass die sogenannte „Flüchtlingskrise“ nicht nur in Deutschland sondern auch in den anderen Mitgliedländern der EU ein Antrieb für die Rechtsextremisten wird (Leggewie, 2017, 115). Sie formulieren, so Lazaridis und Wadia, die Debatte um Flüchtlinge als ein Sicherheitsproblem des Staates um (Lazaridis und Wadia, 2015, 4; Takemura, 2016, 13-14). Obwohl die Rechtspopulisten von der Sicherheit sprechen, muss man, so Takemura, jedoch daran denken, „wessen Sicherheit“ einen Vorrang haben soll. ${ }^{31}$

Die andere „Rechtfertigung“ einer Diskriminierung, welche auf der Kultur oder Allgemeinheit basieren solle, stellt die „liberale“ Logik der Exklusion dar. Die liberalen Werte wie Freiheit, Demokratie, Rechtsstaatlichkeit und Gleichheit zwischen Männern und Frauen werden als die allgemeinen Werte des Aufnahmestaates definiert. ${ }^{32}$ Und die Migranten / Flüchtlinge, die diesen Prinzipien nicht vollständig gerecht zu werden scheinen, werden als diejenigen bezeichnet, die die allgemeinen und liberalen Werte in sich nicht aufnehmen könnten (Orgad, 2010; Sekinou, 2017, 118-120). So wie das Verschleierungsverbot ${ }^{33}$ mit dem liberalen Wert - in diesem Fall, der Trennung von Politik und Religion oder der Gleichheit zwischen Frauen und Männern - ,gerechtfertigt" wird, können davon betroffene Migranten / Flüchtlinge gerade deswegen diskriminiert werden, weil sie die „allgemeinen“ Werte nicht für wichtig halten könnten. ${ }^{34}$

Shakai Shisōshi Kenkyū (Annals of the Society for the History of Social Thought). 42: 114-131 (auf Japanisch), hier, 118.

31 Takemura, 2016, 14. Vgl. Lazaridis und Wadia, 2015, 2.

32 Sekinou Hideaki (2017), „Doitsu ni okeru Torukokei Muslim no shakaiteki haijo: kōkikindai ni okeru national na kyōkai no saikitei“ (,Social Exclusion of Turkish Muslims in Germany: Redefinition of National Boundary in Late Modernity“), in: Mita Shakaigaku (Mita Journal of Sociology). 22: 111-124 (auf Japanisch), hier, 113-115, 119-120; Kashiwazaki, 2016, 48.

33 Vgl. Christian Joppke, Veil: Mirror of Identity. Cambridge: Polity Press, 2009.

34 Vgl. Mizushima Jiro (2014), „Populism to democracy“ (,Populism and Democracy“), in: Chiba Daigaku Hōgaku Ronshū (Chiba Journal of Law and Politics). 29 (1-2): 125-147 (auf Japanisch), hier, 127; Mizushima Jiro (2015), ,'Minshū 


\section{FAZIT}

Ausgehend von einem Vorschlag Leggewies hat sich diese vorliegende Arbeit damit beschäftigt, seinen Vorschlag mit Blick auf die Paradoxa, die sich im Kontext der Migrationspolitik verdeutlichen, zu lesen. Ziel dieses vorliegenden Beitrages besteht aber nicht darin, den Vorschlag Leggewies abzulehnen.

In Zeiten vielseitiger Herausforderungen für die EU muss man weiterhin aufmerksam beobachten, woran sich die EU orientieren muss (Leggewie, 2017). $\mathrm{Zu}$ den dringenden Problemen gehört ohne Zweifel der Umgang mit Flüchtlingen. Hierfür ist eine kritische Aufarbeitung des Begriffs „Grenze“" notwendig.

Es muss in diesem Zusammenhang darum gehen, eine Solidarität innerhalb der EU herzustellen. Man muss dabei darauf aufmerksam machen, dass die Solidarität innerhalb der EU nicht bedeutet, eine dichte materielle Grenze zwischen der EU und anderen Ländern zu ziehen. Auch die soziopsychologischen Grenzen in der Gesellschaft sollen nicht dichter werden (Haba, 2011, 44).

Es bleibt noch schwer zu sehen, wozu die europäische Politik des (Außen-) Grenzschutzes führt. Es ist dabei beispielsweise schwierig einzuschätzen, ob die polizeilichen Kontrollen an den Außengrenzen zur Sicherheit beitragen können (Lazaridis und Wadia, 2015, 3; Takemura, 2016, 12). $\mathrm{Zu}$ beachten ist es aber auch, dass solche Systeme der Begrenzung ,eine Typologie von Insider und Outsider" (Lazaridis und Wadia, 2015, 3; Takemura, 2016, 12) schaffen. Zu Outsider der Grenzen kann man die Flüchtlinge zählen.

Der Zusammenhang zwischen dem Grenzbegriff und der Wahrnehmung der Flüchtlinge als eine „Gefährdung“ verfestigt sich. Man muss sich damit befassen, wie das Trilemma zwischen Menschenrechten, Sicherheit des Staates sowie der EU und liberalen Werten gelöst werden kann.

no daihyō' ka ‘bōekisen' ka: Berugī, Furanderen no populism seitō“ („A Threat

to Democracy?: The Case of the Flemish Populist in Belgium"), in: Chiba Daigaku Hōgaku Ronshū (Chiba Journal of Law and Politics). 29 (4): 1-25 (auf Japanisch), hier, 3-4; Sekinou, 2017, 120-121. 


\section{LITERATUR}

Aas, Katja F. (2011), „Crimmigrant' Bodies and Bona Fide Travelers: Surveillance, Citizenship and Global Governance“, in: Theoretical Criminology. 15 (3): 331-346.

Ausländergesetz vom 28. April 1965, in: Bundesgesetzblatt. Teil I, Nr. 19. (Bonn, am 8. Mai 1965).

Balibar, Etienne (2010), „At the Borders of Citizenship: A Democracy in Translation?", in: European Journal of Social Theory. 13 (3): 315-322.

Eisnecker, Philipp und Schupp, Jürgen (2016), „Flüchtlingszuwanderung:

Mehrheit der Deutschen befürchtet negative Auswirkungen auf Wirtschaft und Gesellschaft“, in: DIW Wochenbericht. 8: 158-164.

Grundgesetz für die Bundesrepublik Deutschland

(https:/www.gesetze-im-internet.de/gg/BJNR000010949.html: Letzter Zugriff: 18. 01. 2019).

Haba, Kumiko (2011), „Kakudai EU ni okeru kyōkaisen to citizenship European Identity to Xenophobia (yosomonogirai) no sōkoku -" ("Border and Citizenship in the Enlarged EU: Conflict between European Identity and Xenophobia"), in: Shakai Shirin (Hosei Journal of Sociology and Social Sciences). 57 (4): 35-53 (auf Japanisch).

Hogan, Jackie und Haltinner, Kristin (2015), „Floods, Invaders, and Parsites: Immigration Threat Narratives and Right-Wing Populism in the USA, UK and Australia“, in: Journal of Intercultural Studies. 36 (5): 520-543.

Joppke, Christian (2009), Veil: Mirror of Identity. Cambridge: Polity Press. Joppke, Christian (2010), Citizenship and Immigration. Cambridge: Polity Press.

Kashiwazaki, Masanori (2016), „Imin seisaku ni okeru liberalism no niitsuhaihan - tōitsu Doitsu no jirei“ (,The Antinomy of Liberalism in German Migration Policy“), in: Tokyo Gaikokugo Daigaku Ronshū (Area and Culture Studies). 92: 45-63 (auf Japanisch).

Kobayashi, Kaoru (2009), „Doitsu no imin seisaku ni okeru 'tōgō no shipai'“ („Germany’s „Failed Integration“ Immigration Policy“), in: Europe Kenkyū (European Studies). 8: 119-139 (auf Japanisch).

Lazaridis, Gabriella und Wadia, Khursheed (2015), „Introduction“, in: Gabriella Lazaridis und Khursheed Wadia (eds.), The Securitisation of 
Migration in the EU: Debates since 9/11. Hampshire: Palgrave Macmillan, 2015, 1-16.

Leggewie, Claus, „Unionsbürgerschaft für Flüchtlinge: EU-Pass als Ausweg aus der Krise“, in: Frankfurter Rundschau. 22. 10. 2015 (http://www.fr.de/politik/unions-buergerschaft-fuer-fluechtlinge-eu-pa ss-als-ausweg-aus-der-krise-a-431867: Letzter Zugriff: 18. 01 2019).

Leggewie, Claus (2017), Europa zuerst! Eine Unabhängigkeitserklärung. Berlin: Ullstein Buchverlag.

Marshall, Thomas H. (1950), Citizenship and Social Class, and Other Essays. Cambridge: Cambridge University Press.

Mizushima, Jiro (2014), „Populism to democracy“ („Populism and DemoCracy"), in: Chiba Daigaku Hōgaku Ronshū (Chiba Journal of Law and Politics). 29 (1-2): 125-147 (auf Japanisch).

Mizushima, Jiro (2015), „,Minshū no daihyō‘ ka ,bōekisen“ ka: Berugī, Franderen no populism seito" ",A Threat to Democracy?: The Case of the Flemish Populist in Belgium“), in: Chiba Daigaku Hōgaku Ronshū (Chiba Journal of Law and Politics). 29 (4): 1-25 (auf Japanisch).

Nakamura, Kengo (2018), „EU wa ekkyō suru hito no kenri o dokomademitometeiruka? EU shimin, imin, nanmin no genjō o fumaete“ („To What Extent Does the European Union Guarantee the Rights of Person Who Crosses Borders?: EU Citizens, Immigrants, and Refugees"), in: The Keizaigaku Zasshi (Journal of Economics). 119 (1): 41-80 (auf Japanisch).

Namba, Satoru (2016), ,EU kokkyō chiiki ni okeru < kyōkai > no politics:Ōshū nanmin kisei regime no kōchiku to Tunisia jin nanmin“ („,Politics of « Borderlands » in the European Union: Construction of a EUropean Immigration Control Regime and Tunisian Refugees after the Arab Spring"), in: Kyōkai Kenkyū (Japan Border Review). 7: 45-70 (auf Japanisch).

Orgad, Liav (2010), „Illiberal Liberalism: Cultural Restrictions on Migrtions and Access to Citizenship in Europe", in: The American Journal of Comparative Law. 58 (1): 53-105.

Paasi, Anssi (1998), „Boundaries as Social Processes: Territoriality in the World of Flows“, in: Geopolitics. 3 (1): 69-88.

Sato, Shigeki (2017), ,Kokumin kokka to gaikokujin no kenri - sengo Doitsu no gaikokujin seisaku kara“ (,Nation-State and the Rights of Foreigners: The Case of the Foreigners Policy in Postwar Germany"), in: 
Shakai Shirin (Hosei Journal of Sociology and Social Sciences). 63 (4): 59-97 (auf Japanisch).

Sekinou, Hideaki (2017), „Doitsu ni okeru Torukokei Muslim no shakaiteki haijo: kōkikindai ni okeru national na kyōkai no saikitei“ (“,Social Exclusion of Turkish Muslims in Germany: Redefinition of National Boundary in Late Modernity"), in: Mita Shakaigaku (Mita Journal of Sociology). 22: 111-124 (auf Japanisch).

Takemura, Nariyoshi (2016), "Nanmin imin tōsei to hanzai tōsei no yūgō oyobi fuhenteki jinken no saikōchiku - kyōkai no kakusan shintō, kanshi tōsei no fuhen kōjōka, kiken / anzen paradigm no datsukōchiku" („Fusion of Refugee / Immigration Control and Crime Control, and Reconstruction of Universal Human Rights: Diffusions-Penetration of Border, Generalization of Surveillance-Control, and Deconstruction of Risk / Security Paradigm“), in: Toin Hōgaku (Toin Law Review). 23 (1): 1-29 (auf Japanisch).

Tsuchiya, Takeshi (2005), „EU to minshuteki citizenship - daisankoku kkumin no hōsetsu o chūshin ni -“ (,The EU and Democratic Citizenship - Focusing on the Inclusion of Third Country Nationals -“), in: Nihon EU Gakkai Nenpō (EU Studies in Japan). 25: 244-266 (auf Japanisch).

Yamaoka, Kenjiro (2018), „Nanmin kenkyū eno shisōshiteki approach liberal na nanminkan o koete“ („Beyond a Liberal View of Refugees“, in: Shakai Shisōshi Kenkyū (Annals of the Society for the History of Social Thought). 42: 114-131 (auf Japanisch). 

Wirtschaft, Recht und Politik der Migration 



\title{
Eine ökonomische Perspektive auf Wanderungsbewegungen und Migrationspolitik
}

\author{
Anna Hochleitner
}

\section{EINLEITUNG}

Die im Jahr 2015 stark gestiegene Zahl der Asylbewerber in der EU ${ }^{1}$ hat nicht nur eine öffentliche Debatte um Asylrecht, humanitäre Verantwortung und europäische Solidarität ausgelöst, sondern auch Diskussionen um arbeitsmarktbezogene Migration neu aufleben lassen. Eine besondere Problematik stellt dabei die Verflechtung von Flucht- und Migrationswegen dar, die zu einer zusätzlichen Belastung des Asylsystems führt und sowohl den Schutz von Flüchtlingen als auch die Entwicklung einer wirksamen Migrationspolitik erschwert. Einer der Gründe für diese Vermischung liegt in restriktiven Visapolitiken und mangelnden legalen Zugangswegen. ${ }^{2}$ Angesichts der hohen Ablehnungsquote von Asylbewerbern und den damit verbundenen Kosten für den Staat könnte eine Öffnung alternativer Zugangswege durch Arbeitsmigration einen Beitrag zur Entflechtung leisten (Ange-

1 Zwischen 2014 und 2015 verdoppelte sich die Anzahl von Asylbewerbern auf etwa 1,3 Millionen. Nach einem geringen Rückgang im Jahr 2016 sanken die Zahlen 2017 weiter auf 700.000 und liegen somit nur knapp über dem Niveau von 2014 (Eurostat, 2018).

2 Siehe dazu den Beitrag von Thea Kirsch in diesem Band. 
nendt et al., 2017). ${ }^{3}$ In Zeiten eines internationalen Wettbewerbes um gut ausgebildete Arbeitskräfte, der durch die Veränderung der demographischen Zusammensetzung vieler Industrieländer an zusätzlicher Bedeutung gewinnt, stellt sich zudem die Frage, wie Zuwanderung effektiver gesteuert werden kann.

Trotz einer zunehmenden Öffnung und Liberalisierung der deutschen Migrationspolitik in den letzten 15 Jahren (Ette et al., 2012), bleibt die tatsächliche Inanspruchnahme legaler Einwanderungsmöglichkeiten wie etwa der Blue Card für hochqualifizierte Migranten hinter ihrem Potential zurück (Klingholz, 2015). ${ }^{4}$ Als Ursache hierfür werden insbesondere die fixen Mindestgehaltsgrenzen gemäß $\S 2$ I Nr. 2 BeschV sowie der komplexe verwaltungstechnische Rahmen genannt. So kommen Hunger \& Krannich (2017) bei einer Analyse der deutschen und europäischen Richtlinien auf 46 unterschiedliche Formen der Erwerbsmigration, was die Unübersichtlichkeit der bestehenden Regelungen unterstreicht. Dieses Ergebnis reiht sich in die Diskussion der letzten Jahre um ein Einwanderungsgesetz ein, das neben einer Vereinfachung der Rechtsnormen eine bessere Vermarktung Deutschlands als Einwanderungsland ermöglichen soll (Langenfeld, 2015). Die kürzliche Einigung der Koalition auf ein Eckpunktpapier für ein Fachkräfteeinwanderungsgesetz (Bundesregierung, 2018) bestätigt die Relevanz und Notwendigkeit der Rejustierung legaler Zuwanderung. ${ }^{5}$

Die Frage nach der konkreten Ausgestaltung der Migrationspolitik eines Landes, schließt zwingenderweise Fragen nach den volkswirtschaftlichen Konsequenzen eines veränderten gesetzlichen Rahmens ein. Derartige Prognosen erfordern eine sorgfältige Analyse aller relevanter Faktoren, die durch die hohe Kontextspezifität einer solchen Entscheidung erschwert wird. Es ist daher nicht verwunderlich, dass trotz häufiger Debatten die öffentliche Meinung beim Thema Migration und Flucht teilweise falschen

3 So lag die Ablehnungsquote einschließlich formeller Entscheidungen für Deutschland im Jahr 2017 bei 56.6\% (BAMF, 2018).

4 Obwohl von den gut 24.000 EU weit ausgestellten Blue Cards im Jahr 2017 knapp $85 \%$ auf Deutschland entfallen, fällt diese Zahl relativ zu den gesamten Immigrationszahlen weiterhin gering aus (Eurostat, 2018).

5 Obwohl sich der vorliegende Beitrag nicht mit einer Analyse des Eckpunktpapiers beschäftigt, werden an verschiedenen Stellen Parallelen gezogen, um einen Bezug zu dieser politischen Diskussion herzustellen. 
Vorurteilen unterliegt. ${ }^{6}$ Schnell wird der Zuwanderung die Schuld an sinkenden Löhnen und Arbeitslosigkeit gegeben, Probleme, von denen in den letzten 20 Jahren insbesondere Arbeitskräfte mit geringerem Bildungsstand betroffen sind. Tatsächlich stellen Studien jedoch langfristig meist keine bis geringe negative Auswirkungen von Zuwanderung auf Löhne und Beschäftiungsniveau fest. Im Falle eines hohen Anteils an hochqualifizierten Migranten sind sogar positive Auswirkungen zu erwarten (Docquier et al., 2014). Andererseits werden mit einer erhöhten Zuwanderung oft zu große Hoffnungen verbunden, vor allem bezüglich ihrer Wirkung auf den demographischen Wandel. Auch sind negative Konsequenzen für bestimmte Regionen und Risikogruppen nicht auszuschließen. Die Analyse potenzieller Konsequenzen, insbesondere auf den Arbeitsmarkt und den Sozialstaat leistet daher einen wertvollen Beitrag zur Diskussion um Zuwanderung. Zudem stellt sich bei der Anwerbung von Fachkräften aus Drittstaaten die Frage nach einer entwicklungspolitischen Verantwortung, da eine intensive Abwanderung von Hochqualifizierten negative Effekte auf die Herkunftsländer haben kann (Docquier \& Rapoport, 2012). Obwohl durch Rücküberweisungen oder zirkuläre Migrationsprogramme auch positive Wirkungsketten entstehen, müssen die Risiken in jedem Fall gründlich begutachtet und in den Entscheidungsprozess mit einbezogen werden.

Der folgende Beitrag soll unter Bezugnahme auf die aktuelle Situation in Deutschland einen empirischen und theoretischen Überblick über die ökonomischen Konsequenzen von Migration ermöglichen. Dabei besteht kein Anspruch auf Vollständigkeit, vielmehr soll die Diskussion aktueller Studien die allgemeine Debatte zur Migrations- und Asylpolitik informieren. Der Beitrag bezieht sich dabei, wenn nicht explizit erwähnt, auf Arbeitsmigration. Aufgrund der Verflechtung zwischen regulärer und irregulärer Migration wird jedoch an verschiedenen Stellen auch Bezug auf die Situationen von Flüchtlingen und Asylbewerbern genommen. Zunächst werden in Abschnitt 2 die Implikationen für Zielländer im Bereich des Arbeitsmarktes, der Fiskalpolitik, des demographischen Wandels sowie die Bedeutung einer schnellen Integration in den Arbeitsmarkt diskutiert. Ab-

6 Dieser Artikel beschränkt sich dabei auf wirtschaftliche Argumente und lässt mögliche soziokulturelle sowie politische und ideologische Konsequenzen außer Acht. Für eine Diskussion dieser Aspekte siehe den Beitrag von Merve Kania und von Kentaro Inagaki in diesem Band. 
schnitt 3 wechselt die Perspektive und beleuchtet mögliche Konsequenzen für die Herkunftsregionen sowie potentielle Konzepte zur Integration einer entwicklungspolitischen Verantwortung in die Migrationspolitik. Abschnitt 4 skizziert schließlich verschiedene Modelle eines Einwanderungsgesetzes. Hierbei werden zunächst nachfrage- und angebotsorientierte Ansätze gegenübergestellt sowie mögliche Synergien zwischen Asyl- und Migrationspolitik diskutiert. Abschnitt 5 fasst die einzelnen Ergebnisse zusammen und schließt ab.

\section{2. ÖKONOMISCHE AUSWIRKUNGEN AUF DIE ZIELLÄNDER}

Die Ängste der Gegner einer offeneren Migrationspolitik beziehen sich häufig auf mögliche Verdrängungs- und Lohneffekte einer stärkeren $\mathrm{Zu}$ wanderung sowie auf fiskalische Belastungen einer Assimilations- und Integrationspolitik. Andererseits werden in Europa zum Teil große Hoffnungen in die Zuwanderung gesetzt, die einen Teil der negativen Konsequenzen des demographischen Wandels abfangen soll. Tatsächlich beeinflusst Immigration die Volkswirtschaft des Aufnahmelandes durch unterschiedlichste Kanäle. Sie verändert die Anzahl und Zusammensetzung der Arbeitskräfte, beeinflusst die Kapitalakkumulation sowie die Güternachfrage und hat Auswirkungen auf den öffentlichen Haushalt, das Steueraufkommen sowie Transferleistungen (Docquier, 2013). All diese Kanäle müssen bei einer Beurteilung der volkswirtschaftlichen Konsequenzen berücksichtigt werden. Außerdem hängen letztere stark von den Charakteristika der Migranten sowie einer schnellen und erfolgreichen Integration in den Arbeitsmarkt ab (Kancs \& Lecca, 2017). Die aus ökonomischer Sicht wohl wichtigsten Faktoren sind dabei die Alters- und Bildungsstruktur der Zuwanderer (Docquier, 2013). Weitere wichtige Aspekte sind die Lohnflexibilität, der Grad der Komplementarität oder Substitutivität, das heißt inwieweit Migranten in ihren Qualifikationen Inländern ähnlich sind oder diese ergänzen, sowie die Schnelligkeit der Anpassung von Kapitalstock und Exporten (Bock-Schappelwein \& Huber, 2015).

Im Allgemeinen ist Migration verbreitet unter jungen, alleinstehenden, qualifizierten Erwachsenen (Kermer, 2007). Ein sehr stark limitierender Faktor bei der Migrationsentscheidung ist das Alter. In der Regel finden in- 
ternationale Wanderungen in einem engen Altersband zwischen 25 und 35 Jahren statt. Menschen über 40 entscheiden sich dabei nur in Ausnahmefällen für diesen Schritt, da insbesondere die immateriellen Kosten der Migration für sie sehr viel höher liegen als für junge Menschen. ${ }^{7}$ Auffallend ist zudem, dass Arbeitsmigranten oft eine überdurchschnittlich hohe Bildung im Vergleich zu ihrem Heimatland besitzen und sogar oft höher qualifiziert sind als die Bevölkerung im Zielland. In OECD Ländern besitzt in etwa ein Drittel der Immigranten einen Universitätsabschluss (Dumont \& Liebig, 2014). Eine mögliche Erklärung hierfür ist, dass für Hochqualifizierte einerseits die Kosten $^{8}$ der Wanderung geringer sind und sie andererseits durch eine selektive Einwanderungspolitik bevorzugt werden (Docquier, 2013). Auch Flüchtlinge sind in Deutschland im Schnitt deutlich jünger als die heimische Bevölkerung, mit einem Anteil der 18-35-Jährigen von 50\%. Bezüglich des Niveaus der Schulbildung herrscht eine große Varietät vor. Während laut einer Befragung des BAMFs 19\% der Asylbewerber in Deutschland eine Universität und $68 \%$ eine weiterführende oder Mittelschule besucht haben, haben 9\% der Befragten gar keine Schule besucht und 26\% die Schulbildung nicht abgeschlossen (Brücker et al., 2016). Eine besondere Problematik bei Geflüchteten ist zudem das Fehlen von Zertifikaten und Urkunden über die jeweiligen Bildungsabschlüsse, weshalb deren Anerkennung deutlich erschwert wird (Brücker et al., 2015).

\subsection{Auswirkungen auf den Arbeitsmarkt}

Grundsätzlich erhöht sich durch Immigration das Arbeitskräfteangebot in einer Volkswirtschaft. Dies erzeugt bei fixen Produktionsmitteln durch eine Veränderung der relativen Knappheiten einen Druck auf aktuelle Durchschnittslöhne und verringert die Beschäftigung inländischer Arbeitnehmer. Allerdings verändert sich durch die Zuwanderung auch die gesamtwirtschaftliche Nachfrage. Kommt es nun zu einer Anpassung des Kapitalstocks durch erhöhte Investitionsanreize oder steigen die Exporte des Landes aufgrund einer erhöhten Wettbewerbsfähigkeit, können Lohn- und Beschäftigungsverluste der Inländer sogar überkompensiert werden (Bock-

7 Insbesondere sinkt die Migrationsbereitschaft deutlich mit der Gründung einer eigenen Familie.

8 Gemeint sind sowohl Kosten materieller als auch immaterieller Art. 
Schappelwein \& Huber, 2015). Empirische Analysen finden in der Tat meist einen vernachlässigbaren Einfluss auf das durchschnittliche Lohnniveau. Während es in kurzer Frist zu geringen negativen Lohneffekten kommen kann (Okkerse, 2008; d'Amuri et al., 2010) kommt es mittel- und langfristig auch zu einer signifikanten Kapitalanpassung, sodass die Löhne langfristig unverändert bleiben (Brücker \& Jahn, 2011). ${ }^{9}$ Was die Beschäftigungswirkung von Zuwanderung betrifft, lassen sich empirisch kaum Verdrängungseffekte feststellen. Meist treten keine negativen Effekte auf und falls doch fallen sie in der Regel gering aus (Docquier, 2013).

Neben Auswirkungen auf das gesamtdurchschnittliche Lohn- und Beschäftigungsniveau ist eine Verringerung der Löhne und der Beschäftigung derer Arbeitskräfte zu erwarten, die sich in einem substitutiven Verhältnis zu den Migranten befinden (Lipsmeyer \& Zhu, 2011). Es ist daher sehr wichtig zu beachten, dass auch wenn der Gesamteffekt der Zuwanderung positiv oder neutral ausfällt, gewisse Bevölkerungsschichten, die enge Substitute für ausländische Arbeitskräfte sind, Wohlfahrtsverlusten ausgesetzt sein können (Kerr \& Kerr, 2011). So können die wirtschaftlichen Gesamteffekte im Widerspruch zur kurzfristigen Realität bestimmter Regionen und Gruppen stehen. Da die Akzeptanz und Toleranz gegenüber Immigranten besonders von der persönlichen Wettbewerbssituation abhängig ist müssen diese Effekte von der Politik berücksichtigt und Risikogruppen unterstützt werden.

Zudem muss eine Unterscheidung zwischen hoch- und geringqualifizierten Zuwanderern vorgenommen werden. Durch die Zuwanderung von geringqualifizierten Arbeitskräften können insbesondere die Durchschnittslöhne für geringqualifizierte Inländer sinken und Verdrängungseffekte ausgelöst werden. Die Möglichkeit eines solchen Szenarios liegt vermutlich den wirtschaftlichen Ängsten vieler Migrationsgegner zugrunde (BockSchappelwein \& Huber, 2015). Es ist daher die Aufgabe der Politik, möglichen negativen Verteilungseffekten rechtzeitig durch Umverteilungsmaßnahmen entgegen zu wirken (Werding, 2015). Anders sieht es bei der Migration von Hochqualifizierten aus. Bei einem hohen Anteil an Hochqualifizierten führt Immigration durch Komplementaritäten zu einem Anstieg der

9 Eine Ausnahme ist Borjas (2003), der unter Verwendung einer abweichenden statistischen Identifikationsstrategie signifikant negative Effekte von Zuwanderung auf das durchschnittliche Lohnniveau feststellt. 
Löhne von Geringqualifizierten und kann durch positive Externalitäten, ${ }^{10}$ die aus dem Anstieg an Humankapital resultieren, auch einen Anstieg der Durchschnittslöhne bewirken (Docquier et al., 2014). So findet Piyapromdee (2017) positive Auswirkungen der Immigration von Hochqualifizierten auf geringqualifizierte Arbeitskräfte, jedoch Wohlfahrtsverluste für hochqualifizierte Inländer. Eine Analyse der OECD Länder bestätigt, dass Immigration zwischen 1990 und 2000 die Löhne von geringqualifizierten Arbeitnehmern signifikant erhöht und so zu einer Verringerung der Lohnungleichheit beigetragen hat (Docquier et al., 2014). ${ }^{11}$ Ähnlich finden Ottaviano \& Peri (2012) einen geringen positiven Effekt von Zuwanderung auf das durchschnittliche Lohnniveau von geringqualifizierten Inländern in den USA. Für vorherige Immigranten hingegen, verringern sich die Löhne durch weitere Zuwanderung.

Während geringqualifizierte Migranten und inländische Arbeitskräfte oft weitgehend austauschbar sind, löst die Zuwanderung von Hochqualifizierten laut Studien in der Regel kaum Verdrängungsprozesse aus (Kermer, 2007) und kann sogar zu positiven Beschäftigungswirkungen führen (Docquier, 2013). Der Grund hierfür ist, dass die Nachfrage nach qualifizierten Arbeitskräften in Industrieländern durch eine zunehmende Technologisierung und stärkere Spezialisierung weiterhin steigt, während das Angebot, bedingt durch den demographischen Wandel, ständig sinkt (Boeri et al., 2012). In diesem Zusammenhang stellt die Zuwanderung von Hochqualifizierten ein bedeutendes Potential dar. Darüber hinaus stellen hochqualifizierte Migranten in unserer auf Wissensaustausch basierten Gesellschaft eine treibende Kraft für Innovation und Fortschritt dar und erweitern somit die Produktionsmöglichkeitengrenze des Landes (Chiswick, 2005). Aus diesen Gründen soll durch die Umsetzung der Blue Card Richtlinie (RL 2009/50/EG) die Einwanderung von Hochqualifizierten durch die Schaffung eines eigenen Aufenthaltstitels gezielt gefördert werden (siehe §19a AufenthG). Wie bereits erwähnt bleibt der Erfolg dieser Maßnahme jedoch

10 Unter Externalitäten versteht man positive oder negative Nebeneffekte wirtschaftlicher Entscheidungen, von denen außenstehende Dritte betroffen sind und die nicht vollständig durch Preise abgebildet werden (Bishop, 2004).

11 Dieses Ergebnis bleibt robust, auch nachdem Bildungsabschlüsse zum Teil nach einem internationalen Vergleich abgewertet wurden. 
auf Grund weiterhin bestehender rechtlicher und verwaltungstechnischer Hürden begrenzt.

Trotz der besonderen volkswirtschaftlichen Bedeutung von Humankapital, hat die Bundesrepublik nicht ausschließlich ein Interesse an der $\mathrm{Zu}$ wanderung von hochqualifizierten Fachkräften. Insbesondere in Branchen wie der Gastronomie, der Landwirtschaft oder der Pflege herrscht gegenwärtig eine hohe Nachfrage nach Arbeitskräften (Brücker et al., 2015). Füllen Migranten die Nischen, die von inländischen Arbeitskräften nicht besetzt sind, besteht ebenfalls ein geringeres Niveau an Substitution (Dumont \& Liebig, 2014). ${ }^{12}$ Obwohl die derzeitige Gesetzeslage die Einwanderung von Migranten mit einer Ausbildung in Mangelberufen durch das entfallen rechtlicher Hürden, wie beispielsweise der Vorrangprüfung erleichtert (siehe $§ 6$ BeschV), fallen die Regelungen für diese Gruppe im Vergleich zur Migration von Hochqualifizierten noch immer relativ restriktiv aus. ${ }^{13}$ Dies soll sich laut dem neuen Eckpunktpapier der Koalition nun auch für Fachkräfte mit qualifizierter Berufsausbildung ändern. ${ }^{14}$ Dies heißt zum einen die Gewährung eines Aufenthaltsrechtes für 6 Monate zum Zweck der Arbeitssuche, zum anderen ein Verzicht auf die Vorrangprüfung (Bundesregierung, 2018).

Insgesamt lässt sich mit Hinblick auf den Arbeitsmarkt also feststellen, dass die Auswirkungen von Zuwanderung tendenziell gering und oft nicht signifikant ausfallen. Auch durch die verstärkte Zuwanderung von Asylbewerbern nach Deutschland sind Studien zufolge keine langfristigen Auswirkungen auf das Lohnniveau und nur geringe negative Konsequenzen für das Beschäftigungsniveau im Rahmen von maximal $-0.07 \% \mathrm{zu}$ erwarten (Brücker et al., 2015).

12 Schon jetzt führt ein großer Bedarf an Fachkräften jeglichen Ausbildungsniveaus und Spezialisierungsgrades $\mathrm{zu}$ einer europaweiten Rekrutierung von Fachkräften in einigen Branchen (siehe z.B. Simon, 2011 oder Demary \& Erdmann, 2012).

13 Für eine genauere Diskussion der rechtlichen Grundlagen siehe den Beitrag von Nils Irmgarten in diesem Band.

14 Welches Ausbildungsniveau vorgewiesen werden muss ist jedoch derzeit noch unklar (Bader \& Steffen, 2018). 


\subsection{Auswirkungen auf die Fiskalpolitik}

Eine weitere Befürchtung vieler Bürger ist, dass durch die finanzielle Unterstützung von Flüchtlingen und Migranten der Staatshaushalt belastet und eigene Sozialleistungen gekürzt werden könnten. Besonders da der Wohlfahrtsstaat in Aufnahmeländern oft stärker ausgebaut ist als in vielen Herkunftsländern, warnen Politiker vor einer Zuwanderung in die sozialen Sicherungsnetze. Empirisch gesehen besteht bezüglich dieser Frage eine große länderspezifische Diversität. Die frühesten Studien zu diesem Thema stammen aus den USA und zeigen, dass Immigranten dort im Vergleich zu Nichtmigranten seltener Sozialleistungen in Anspruch nehmen. Tun sie es doch, so ist die Intensität der Nutzung ähnlich zu der von Inländern (Blau, 1984). Spätere Analysen zeigen in den USA jedoch einen Anstieg der Inanspruchnahme von Sozialleistungen (Borjas \& Trejo, 1991). In Europa sind Migranten Studien zu Folge relativ häufiger auf Sozialleistungen angewiesen als Inländer. Dabei muss allerdings beachtet werden, dass Migranten oftmals der Zugang zum Arbeitsmarkt durch mangelnde Sprachkenntnisse, eine fehlende Anerkennung von Qualifikationen oder Diskriminierung erschwert wird (Kerr \& Kerr, 2011). So zeigen Büchel \& Frick (2005) in einer paneuropäischen Analyse, dass insbesondere in Dänemark und Deutschland Migranten aus ökonomischer Sicht deutlich hinter der inländischen Bevölkerung zurückfallen.

Was das allgemeine Niveau der Sozialleistungen betrifft lässt sich ein vernachlässigbarer Einfluss der Öffnung des Arbeitsmarktes feststellen. Kürzungen von Sozialleistungen als Reaktion auf eine gestiegene Zuwanderung lassen sich empirisch nicht bestätigen. Vielmehr scheint der Umgang der Politik mit Immigration entscheidend zu sein. So sind höhere Immigrationsraten mit höheren Sozialleistungen verbunden, wenn linke Parteien im Parlament eine Mehrheit haben und Gewerkschaften eine relativ starke Machtposition besitzen. In einem rein neoliberalen Szenario mit schwachem Arbeitnehmerschutz hingegen kann Immigration zu einer Senkung von Sozialleistungen führen (Lipsmeyer \& Zhu, 2011).

Aus fiskalpolitischer Sicht stellt sich letztendlich die Frage ob die durch die Migranten verursachten Kosten in Wohlfahrts-, Bildungs- und Gesundheitssystemen durch die steuerlichen Zusatzeinnahmen gedeckt werden können. Die Mehrzahl der Studien zeigt hierbei, dass die fiskalische Bilanz von Zuwanderung in OECD Ländern relativ zum BIP meist geringfügig po- 
sitiv ausfällt (Docquier, 2013). Bonin (2002) zeigt in einer empirischen Analyse der fiskalischen Gesamtwirkungen für Deutschland, dass Zuwanderung zur Entlastung des öffentlichen Haushaltes beitragen kann. Voraussetzung hierfür ist jedoch eine schnelle Integration in den Arbeitsmarkt und das Erreichen einer mit der restlichen Bevölkerung vergleichbaren fiskalischen Leistungsfähigkeit. In den wenigen Fällen, in denen der Gesamteffekt negativ ausfällt, liegt der Grund hierfür nicht an in größerem Umfang beanspruchten Sozialleistungen, sondern an der Tatsache, dass Migranten oftmals geringere Löhne beziehen als Inländer und so weniger Steuern beitragen (Dumont \& Liebig, 2014). Auf individueller Ebene ist der fiskalische Nettoeffekt abhängig von Charakteristika wie Alter und Bildungsstand. Hochqualifizierte Immigranten bringen nicht nur zusätzliches Humankapital ins Land, sondern stärken das Sozialsystem und verringern die allgemeine Steuerbelastung, da sie in der Regel durch ein hohes Einkommen zur Entlastung des Fiskus beitragen (Chiswick, 2005). Migranten mit schlechtem Bildungsstand und ältere Menschen hingegen verursachen dem Staat im Allgemeinen höhere Kosten. Neben Rentnern entstehen vor allem für Kinder hohe Ausbildungs- und Sozialkosten, weshalb aus fiskalpolitischer Sicht Migranten zwischen 20 und 30 Jahren besonders attraktiv sind (Kerr \& Kerr, 2011). Insgesamt gesehen bestätigen sich aus fiskalischer Perspektive weder die Ängste einer Ausbeutung des Sozialstaates noch die Hoffnung auf eine Lösung der fiskalischen Probleme des demographischen Wandels.

\subsection{Demographischer Wandel und langfristige Arbeitsnachfrage}

Die aktuelle Debatte um Zuwanderung ist eng mit den zu erwartenden gesellschaftlichen und wirtschaftlichen Veränderungen im Rahmen des demographischen Wandels verknüpft. Viele Industrieländer stehen durch sinkende Fertilitäts- und Sterblichkeitsraten vor einer neuen sozialen Realität (Findlay \& Wahba, 2013). Problematisch ist hierbei insbesondere das Phänomen der alternden Gesellschaft, mit dem in Zukunft weitgehende sozioökonomische Probleme verbunden sein werden. Während 1960 nur etwa 17\% der deutschen Bevölkerung über 61 war, waren es im Jahr 2013 bereits 27\%. Prognosen zu Folge ist ein Anstieg dieser Altersgruppe auf über $37 \%$ bis zum Jahr 2050 zu erwarten. Der Anteil der unter 20-Jährigen hin- 
gegen soll bis 2050 auf etwa 16\% sinken, was einer Halbierung im Vergleich zu 1960 entspricht (Statistisches Bundesamt, 2015). Auch auf europäischer Ebene werden die meisten Länder in den nächsten Jahren mit einer sinkenden und alternden Bevölkerung konfrontiert sein während die globale Bevölkerung weiterwächst (Bermingham, 2001). ${ }^{15}$ Dies zeigt, dass die Problematik nicht durch europäische Binnenmigration gelöst werden kann.

Um angesichts der veränderten Altersstruktur das aktuelle Produktivitätsniveau zu erhalten, ist eine Reihe an Maßnahmen nötig, die das Erwerbspersonenpotential steigern. Hierunter fallen sowohl eine bessere Integration von Frauen und älteren Beschäftigten in den Arbeitsmarkt, als auch eine gezielte Förderung von Zuwanderung, da das inländische Potential zur Deckung des Arbeitskräftebedarfs nicht ausreichen wird (Klingholz, 2015). Selbst bei einer abrupten und nachhaltigen Steigerung der Fertilitätsrate können die demographischen Probleme Europas in den nächsten 50 Jahren nicht gelöst werden. Eine verstärkte Zuwanderung hingegen kann einen signifikanten und sofortigen Beitrag zur Linderung des Bevölkerungsrückganges leisten, den Arbeitskräftemangel verringern und sich positiv auf das Gesundheitssystem auswirken (Aiyar et al., 2016). Allerdings können die mit der Alterung der Bevölkerung verbundenen Probleme auch durch Migration nicht vollständig aufgefangen werden. Obwohl Migranten meist relativ jung sind, wären zur Stabilisierung der Arbeitsbevölkerung unrealistisch hohe Zuwanderungsraten nötig (Bermingham, 2001).

Insgesamt betrachtet ist Zuwanderung also kein Allheilmittel für die sozioökonomischen Probleme des demographischen Wandels, kann jedoch einen Beitrag $\mathrm{zu}$ deren Linderung leisten. Als Folge dessen sowie auf Grund eines verstärkten globalen Wettbewerbs stehen Industrieländer in zunehmender Konkurrenz um internationale Fachkräfte (Boeri et al., 2012). Entscheidend ist hierbei, dass Migration heute nicht mehr als einmaliger, unidirektionaler Ortswechsel gesehen werden kann, sondern sich durch grenzüberschreitende Netzwerke und Diasporagemeinden zu einem sich

15 Neben Deutschland ist die Situation besonders gravierend in Finnland, Italien und Griechenland. Derzeit noch weniger stark vom Alterungsprozess der Bevölkerung betroffen sind Frankreich, Irland, Island, Malta und Norwegen. Prognosen zu Folge werden sich jedoch in den nächsten 25 Jahren nur in Irland und Island relativ günstige Voraussetzung bezüglich der Bevölkerungsstruktur halten können (Demary und Erdmann, 2012). 
selbst erhaltenden kumulativen Prozess entwickelt (Oltmer, 2012). Durch die erleichterte Übermittlung von Wissen und Informationen über das Zielland wandern Menschen oft in Regionen, zu denen bereits soziale Kontakte bestehen, wodurch ein Zusammenhang zwischen individueller Migrationsentscheidung und Kettenmigrationsprozessen entsteht (Geiger \& Steinbrink, 2012). Dies bedeutet, dass Staaten, um zukünftig von Zuwanderung profitieren zu können, diese schon heute fördern müssen (Werding, 2015).

\subsection{Bedeutung einer schnellen Integration in den Arbeitsmarkt}

Wie bereits erwähnt ist die Realisierung vieler mit Zuwanderung verbundener Vorteile von einer schnellen Integration in den Arbeitsmarkt abhängig. Allerdings sehen sich Migranten in der Regel mit Barrieren konfrontiert, die es ihnen erschweren wirtschaftlich erfolgreich zu sein (Dancygier \& Laitin, 2014).$^{16}$ In Deutschland verdienen Zuwanderer bei ihrer Ankunft etwa $20 \%$ weniger als Inländer mit vergleichbaren Qualifikationen. Der Einkommensunterschied nimmt zwar mit der Zeit ab, es kommt jedoch in der Regel zu keiner vollständigen Angleichung. Dieser Assimilationsprozess ist neben dem Bildungsstand der Migranten auch von den ökonomischen Bedingungen im Aufnahmeland zum Zeitpunkt der Immigration abhängig (Aiyar et al., 2016). Des Weiteren fällt die Arbeitsmarktpartizipation in der Regel im Vergleich zu Inländern geringer aus während gleichzeitig ein relativ höheres Arbeitslosigkeits- und Armutsrisiko besteht (Kerr \& Kerr, 2011).

Zudem zeigen Studien, dass Migranten oftmals für ihre Tätigkeiten überqualifiziert sind und niedrigere Löhne als heimische Arbeitskräfte mit demselben Bildungsniveau erhalten. Ein Grund hierfür ist oftmals ein Scheitern im internationalen Anerkennungsprozess von Bildungstiteln. ${ }^{17}$

16 Problematisch sind hierbei nicht nur sprachliche und kulturelle Barrieren, die Migranten auf dem Arbeitsmarkt benachteiligen, sondern auch eine Erschwerung des Zugangs durch offene oder implizite Diskriminierung (Kerr \& Kerr, 2011).

17 Gehen Migranten einer Tätigkeit unter ihrem eigentlichen Ausbildungsniveau nach, wird in diesem Zusammenhang in der Literatur auch von einem brain waste gesprochen (siehe z.B. Mattoo et al., 2008). 
Dies ist nicht nur für die Migranten selbst problematisch, sondern auch für das Aufnahmeland, da so fachliches Potential ungenutzt bleibt und zukünftige Migrationsentscheidungen negativ beeinflusst werden können (von Hausen, 2010). ${ }^{18}$

Auch wenn sich die Unterschiede zwischen Migranten und Nichtmigranten über die Zeit verringern, ist das bestehende ökonomische Ungleichgewicht vor allem in Westeuropa beunruhigend und stellt eine Herausforderung für die Politik dar (Dumont \& Liebig, 2014). Maßnahmen, die auf eine bessere Arbeitsmarktintegration der Zuwanderer abzielen, sollten daher als Investitionen und nicht als Kosten gesehen werden. Kancs \& Lecca (2017) untersuchen in einer Simulation die Auswirkungen einer Erhöhung der Ausgaben im Bereich der Integrationspolitik, um besonders Flüchtlingen einen besseren Zugang zum Arbeitsmarkt zu ermöglichen. Ihre Ergebnisse zeigen, dass solche Maßnahmen langfristig zu einem höheren BIP Wachstum führen und eine positive Fiskalbilanz aufweisen, sodass eine bessere Integration langfristig nicht nur zu sozialen, sondern auch zu wirtschaftlichen Gewinnen führt. Obwohl die kurzfristigen Kosten solcher Maßnahmen durchaus beträchtlich sind, dürften die langfristigen Kosten einer gescheiterten Integration diese bei weitem übersteigen.

\section{3. ÖKONOMISCHE AUSWIRKUNGEN AUF DIE HERKUNFTSLÄNDER}

\subsection{Die Brain-Drain-Problematik}

Im Mittelpunkt der politischen Debatte um Migration stehen meist Auswirkungen auf das Zielland und der Versuch, durch eine aktive Steuerung von Zuwanderung zu profitieren. Demgegenüber steht eine politische Verantwortung gegenüber den Herkunftsländern, vor allem wenn es sich bei diesen um Schwellen- und Entwicklungsländer handelt. Während Zielländer durch die Immigration von Hochqualifizierten durch vielfache Kanäle profitieren können, führt sie gleichzeitig zu einem Verlust an Humankapital im

18 Diese Problematik findet auch im aktuellen Eckpunktpapier für ein Fachkräfteeinwanderungsgesetz Erwähnung (Bundesregierung, 2018). 
Herkunftsland, der in der Literatur als brain drain bezeichnet wird (Docquier \& Rapoport, 2012).

Der Begriff geht auf die Phase starker Abwanderung von Fachkräften und Wissenschaftlern in die USA nach dem Zweiten Weltkrieg zurück. Während frühe wissenschaftliche Abhandlungen davon ausgingen, dass die Auswirkungen des brain drain auf die Herkunftsländer der Migranten neutral seien und insbesondere die Vorteile freier Migrationsströme für die Weltwirtschaft betont wurden (siehe z.B. Grubel \& Scott, 1966 oder Mishan \& Needlean, 1968), fand später eine Fokussierung auf negative Konsequenzen des brain drains statt, bei der vor allem Entwicklungsländer im Zentrum der Diskussion standen. ${ }^{19}$ Der Verlust an qualifizierten Arbeitskräften führt dabei nicht nur zu einer Verringerung des Humankapitals in den Herkunftsländern, sondern darüber hinaus zu einem Wegfall positiver Externalitäten. So entscheiden sich hochqualifizierte Individuen mit einer höheren Wahrscheinlichkeit für unternehmerische Tätigkeiten, was zu einer Schaffung von Arbeitsplätzen führt, und tragen sowohl in besonderem Maße zu wirtschaftlicher Innovation als auch zu politischen Debatten bei (Langthaler \& Hornoff, 2008). Ein weiteres Problem entsteht, falls Bildung teilweise oder mehrheitlich durch Steuern finanziert wird, da die Herkunftsländer so die Kosten der Ausbildung tragen ohne von späteren Steuerzahlungen zu profitieren (Gibson \& McKenzie, 2010). ${ }^{20}$

Aus diesen Gründen besteht die Sorge, dass brain drain die Perspektiven auf Wachstum und Entwicklung für Herkunftsländer deutlich verschlechtert. Besonders befürchtet werden Personalknappheiten in strategischen Sektoren wie dem Gesundheits- oder Bildungswesen (Kingma, 2018; Langthaler \& Hornoff, 2008). Auch wenn diese Effekte abgeschwächt werden können, falls Hochqualifizierte in ihrem Heimatland arbeitslos sind oder keine adäquate Beschäftigung finden können (Kermer, 2007) kann

19 Eine interessante Tendenz in diesem Zusammenhang ist, dass Migrationsprozesse oft vom Mittelstand eines Landes initiiert werden, der entscheidend zur wirtschaftlichen Entwicklung eines Landes beiträgt (Pries, 2001; Waldrauch, 1995).

20 Diese Problematik könnte durch eine Förderung von Migration zu Ausbildungszwecken verringert werden. 
Emigration auch in diesem Fall schädlich sein, wenn sie interne Verteilungseffekte verhindert (Bhagwati \& Rodriguez, 1975). ${ }^{21}$

Seit den 1990ern werden Bedingungen diskutiert, unter denen sich ein anfänglicher brain drain positiv auf die Herkunftsregionen auswirken kann (Docquier \& Rapoport, 2012). Ein zentrales Argument ist hierbei, dass durch die Migration von Hochqualifizierten die Rendite für Humankapital steigt und somit individuelle Bildungsanreize im Herkunftsland geschaffen werden (Mountford, 1997). Wenn nur ein Bruchteil nach der Ausbildung auch tatsächlich emigriert, steigt somit das Humankapitalniveau der Herkunftsregion (Beine et al., 2001). Dieser Effekt wird jedoch dadurch eingeschränkt, dass auch geringqualifizierte Arbeiter emigrieren, steigende staatliche Bildungsinvestitionen zu einem Rückgang anderer öffentlicher Güter führen können und Bildungstitel im Ausland eventuell nicht vollwertig anerkannt werden, was oftmals zu beruflicher Dequalifizierung führt (Özden $\&$ Schiff, 2006). Wenn sich in- und ausländische Arbeitsmarktbedürfnisse unterscheiden können zudem Verzerrungen im Bereich der Bildungsentscheidung auftreten. Wird die Studienentscheidung auf Bedürfnisse des internationalen statt des inländischen Arbeitsmarktes ausgelegt, können Herkunftsländer nicht in vollem Umfang von dem gestiegenen Humankapital profitieren (Docquier \& Rapoport, 2012).

Ein weiterer Punkt, der die negativen Konsequenzen der Abwanderung lindert, sind die Rücküberweisungen, mit denen Migranten ihre Familien in der Heimat unterstützen. Diese tragen zu einer Entlastung des Staatsbudgets bei und können einen wesentlichen Beitrag zur Entwicklung eines Landes leisten. $^{22}$ Darüber hinaus unterstützen Diasporanetzwerke oftmals über Technologie- und Wissenstransfers eine wirtschaftliche Vernetzung von Herkunfts- und Zielländern und können so Handel und Direktinvestitionen erhöhen (Gibson \& McKenzie, 2010).

21 Da im Kontext internationaler Wanderungen, große Städte in Herkunfts- sowie in Zielländern besonders betroffen sind, kann Emigration arbeitslose Fachkräfte in Städten davon abhalten in ländliche Gebiete abzuwandern, was Verteilungsprobleme innerhalb eines Landes verstärkt.

22 So erreichten Rücküberweisungen in den zehn Hauptempfängerländern 2014 Werte zwischen 20 und $40 \%$ des BIPs (Ratha et al., 2016). 


\subsection{Empirische Evidenz und Lösungsansätze}

In der Tat sind nicht nur die Vorhersagen theoretischer Modelle, sondern auch die Ergebnisse empirischer Analysen zum Thema brain drain gemischt. Studien zu Folge sind besonders kleine Länder von den negativen Konsequenzen des brain drains betroffen ${ }^{23}$ (Beine et al., 2008), während größere Länder mit mittlerem Einkommen profitieren können (Docquier, 2013). Neben der Größe des Landes entscheidet vor allem die Höhe der Abwanderungsrate, ob die Abwanderung eine Chance oder einen Schaden für das Herkunftsland darstellt. Bei einer begrenzten Abwanderungsrate von fünf bis zehn Prozent der qualifizierten Arbeitskräfte ist eine positive Rückwirkung wahrscheinlich. Problematisch wird es, wenn diese über zehn Prozent liegt (Langhoffer \& Hornoff, 2008). ${ }^{24}$

Während klassische Migrationstheorien in der Regel eine einmalige, unidirektionale Auffassung von Migration vertreten, sind im 21. Jahrhundert zirkuläre und temporäre Migrationsformen von zunehmender Bedeutung. Während man unter temporärer Migration einen Auslandsaufenthalt für einen begrenzten Zeitraum versteht, ${ }^{25}$ bezieht sich zirkuläre Migration auf eine kontinuierliche Bewegung zwischen verschiedenen Ländern und kann sowohl temporäre als auch längerfristige Aufenthalte umfassen (IOM, 2008). Beide Migrationsformen werden mit positiven Auswirkungen auf Herkunfts- und Aufnahmeländern verbunden. Zurückkehrende Migranten stellen dabei ein besonderes Potential für Herkunftsländer dar, da diese durch die Übertragung von neu erlerntem explizitem und implizitem Wissen ihre Heimatregion wirtschaftlich voranbringen können (Sinyolo, 2012). Zentral ist dabei, dass Remigranten bei ihrer Rückkehr aktiv unterstützt werden, da sie sich oftmals in ihrem Heimatland erneut mit verschiedenen Hindernissen konfrontiert sehen. Ein Hauptproblem ist, dass lokale Akteure Remigranten oftmals auf Grund der im Ausland erworbenen Verhaltens-

23 Am stärksten von den negativen Folgen des brain drain betroffen sind arme, kleine, englischsprachige Länder im Bereich der Tropen (Docquier, 2013).

24 Global betrachtet scheint es unter den Entwicklungsländern mehr Verlierer als Gewinner zu geben. Allerdings befinden sich unter den letzteren die großen Schwellenländer (Brasilien, Indien, China), so dass in Zahlen ausgedrückt die Gewinne die Verluste der Verliererländer überwiegen (Beine et al., 2008).

25 In der Regel geht man hierbei von einer Zeitspanne von über einem Jahr aus. 
weisen mit Misstrauen begegnen. Dieses muss erst überwunden werden, um einen effektiven Wissenstransfer zu ermöglichen (Kermer, 2007; Schaland, 2012).

Aufgrund des besonderen Potentials von Remigranten für die Herkunftsländer stellen bilaterale Abkommen, die zirkuläre und temporäre Migrationsmuster fördern sollen, eine häufig diskutierte nachhaltige Alternative dar. Oft wird in diesem Zusammenhang von einer triple win Situation gesprochen, bei der sowohl Migranten, als auch Herkunfts- und Zielländer profitieren sollen (Schneider \& Parusel, 2011). ${ }^{26}$ Neben der Erleichterung spontaner beziehungsweise natürlicher zirkulärer Migration können konkrete Anreize zur Remigration, wie beispielsweise die Rückerstattung von Sozialversicherungsbeiträgen oder eine aktive Unterstützung bei der Rückkehr gesetzt werden (Martin, 2004). Remigration setzt jedoch in der Regel vermehrt erst ein, wenn im Heimatland wirtschaftliche und politische Fortschritte erzielt werden, was die Förderungsmöglichkeiten in der Praxis einschränkt. Aus diesem Grund sind parallel die Stärkung nationaler Ausbildungssysteme und eine Verbesserung der allgemeinen Arbeitsbedingungen vor Ort von zentraler Bedeutung (Langthaler \& Hornoff, 2008).

\section{GESTALTUNG EINES EINWANDERUNGSGESETZES}

\subsection{Angebots- und nachfrageorientierte Ansätze}

Grundsätzlich kann in der Migrationspolitik zwischen angebots- und nachfrageorientierten Ansätzen unterschieden werden (EMN inform, 2015). Während erstere dem Faktor Humankapital eine besondere Bedeutung zuschreiben, erfolgt bei nachfrageorientierten Ansätzen eine strenge Rückkopplung an den Arbeitsmarkt. Das bedeutet, dass zentral für das Erlangen einer Aufenthaltserlaubnis der Nachweis eines Arbeitsvertrages ist. Dieser

26 Ein klassisches Beispiel eines so genannten beneficial brain drains ist die indische IT- Branche, die sich erst durch Remigration und die damit verbundenen Wissens- und Technologietransfers zu einem tragenden und dynamischen Wirtschaftszweig entwickeln konnte (Hunger, 2003). 
Ansatz wird bis auf wenige Ausnahmen, die eine Einreise zur Arbeitsplatzsuche erlauben, traditionell in Deutschland verfolgt (Hunger \& Krannich, 2017). ${ }^{27}$ Wie bereits erwähnt sieht das Eckpunktpapier eine Lockerung dieser Regelung für qualifizierte Fachkräfte vor, die nun wie für Akademiker einen Aufenthalt von maximal sechs Monaten zur Arbeitsplatzsuche zulassen soll (Bundesregierung, 2018). Allerdings richten sich die neuen Richtlinien explizit weiterhin am Bedarf der deutschen Volkswirtschaft aus. Während ein großer Vorteil nachfrageorientierter Ansätze in einer schnellen Arbeitsmarktintegration liegt (Langenfeld, 2015), wird sie von anderen als unflexibel und als für Migranten unattraktiv kritisiert (Hunger \& Kranich, 2017).

Ein Beispiel für eine angebots- und somit humankapitalorientierte Migrationspolitik sind punktebasierte Systeme, die in traditionellen Einwanderungsländern wie Australien, Neuseeland oder Kanada $\mathrm{zu}$ finden sind (Wrobel, 2016). ${ }^{28}$ Dabei müssen Migranten eine nach verschiedenen Kriterien errechnete Punktzahl erreichen, um einen Aufenthaltstitel zu erlangen (siehe z.B. Hunger \& Krannich, 2017 oder Werding, 2015). Ein großer Vorteil dieses Ansatzes ist es, dass dabei sowohl demographische sowie arbeitsmarktrelevante Faktoren berücksichtigt werden können. Wie oben diskutiert hängt eine Vielzahl der fiskalpolitischen und arbeitsmarktbezogenen Konsequenzen der Zuwanderung von den individuellen Charakteristika der Migranten ab. So sind vor allem Alter und Bildungsstand von zentraler Bedeutung. Durch eine gezielte Förderung der Migration Hochqualifizierter, wie beispielsweise Erleichterungen bei der Einbürgerung oder der Familienzusammenführung sowie eine selektive Einwanderungspolitik sollen so die Gewinne aus der Zuwanderung für das Zielland maximiert werden (Kermer, 2007). Ein weiterer Vorteil punktebasierter Systeme liegt in einer Flexibilisierung von Migrationswegen sowie in einem Abbau institutioneller Hürden und bürokratischer Komplexität. Die zentrale Schwäche eines

27 Der Aufenthalt zur Arbeitsplatzsuche für Ausländer mit deutschem oder vergleichbarem Hochschulabschluss für eine Dauer von sechs Monaten ist im $§ 18 \mathrm{c}$ I AufenthG geregelt.

28 Aufgrund länderspezifischer Unterschiede hinsichtlich Migrationsrouten und gesamtwirtschaftlicher Faktoren ist ein direkter Vergleich zwischen Ländern problematisch. Unter Berücksichtigung dieser Limitierung können internationale Erfahrungen jedoch hilfreiche Orientierungspunkte bieten. 
solchen Ansatzes ist eine verschlechterte Arbeitsmarktintegration (Langefeld, 2015). Aus diesem Grund kam es in den letzten Jahren auch in Ländern wie Kanada, dem klassischen Beispiel für ein humankapitalorientiertes Punktesystem, zu steuerungssystematischen Anpassungen und einer Integration nachfrageorientierter Elemente (Langefeld, 2015).

Eine viel diskutierte Lösung ist daher eine Kombination von sowohl angebots- als auch nachfrageorientierten Elementen im Rahmen eines Hybridmodells (siehe z.B. Angenendt et al., 2017 oder Wrobel, 2016). Aufgrund der Öffnung Deutschlands insbesondere für hochqualifizierte Migranten innerhalb der letzten Jahre, wird zudem argumentiert, dass der aktuelle rechtliche Rahmen die Integration humankapitalorientierter Elemente nicht nur ermöglicht, sondern bereits begonnen hat (Langenfeld, 2015). Dadurch wird die Umwandlung in ein Punktesystem nicht nur unnötig, eine Dopplung bestehender Zugangsmöglichkeiten birgt zudem die Gefahr einer erhöhten Intransparenz und Bürokratie (Langenfeld, 2015). Stattdessen werden in dem aktuellen Eckpunktpapier innerhalb des bestehenden rechtlichen Rahmens bürokratische Vereinfachungen sowie eine Liberalisierung für qualifizierte Fachkräfte angestrebt. In jedem Fall wird eine bessere Vermarktungsstrategie Deutschlands als Einwanderungsland im internationalen Wettbewerb um Fachkräfte dringend benötigt. Ein Einwanderungsgesetz kann den Rahmen dazu bieten und gleichzeitig ein Bekenntnis zu Offenheit signalisieren (Hinte et al., 2011; Klingholz, 2015; Langenfeld, 2015; Straubhaar, 2015).

Eine Teilgruppe der Migranten, die in Einwanderungsgesetzen in der Regel eine besondere Berücksichtigung findet sind Auszubildende und Studierende. Eine zentrale Problematik der Arbeitsmarktintegration ist, dass Bildungsabschlüsse oft international nur schwer vergleichbar sind oder dass es Migranten an komplementären Fähigkeiten, wie Sprachkenntnissen und kulturellem Wissen fehlt. In Folge dessen gehen sie oft einer Tätigkeit nach, die nicht ihren Qualifikationen entspricht. Ein vielversprechender Lösungsansatz ist es, den Schritt ins Ausland bereits während der Ausbildung zu vollziehen (Boeri et al., 2012). ${ }^{29}$ Aufnahmeländer können in diesem Fall von der Akquirierung an neuem Humankapital profitieren, das bereits gut

29 So liegt das Einkommen von Immigranten, die ihren Hochschulabschluss bereits im Zielland erworben haben in der Regel sogar signifikant über dem Lohnniveau inländischer Absolventen (Boeri et al., 2012). 
an die Bedingungen auf dem heimischen Arbeitsmarkt angepasst ist. Von besonderem Interesse sind hierbei die Zulassung und Anwerbung internationaler Studierender. Zudem besteht empirisch eine enge Korrelation zwischen der Mobilität der Studierenden eines Landes und der späteren Migrationsrate (Felbermayr \& Reczkowski, 2012).

Viele Länder haben dies inzwischen erkannt und bemühen sich, die Einreisemöglichkeiten für ausländische Studierende zu verbessern und ihnen nach ihrem Abschluss den Einstieg in den Arbeitsmarkt zu erleichtern (Boeri et al., 2012). Aufgrund des bestehenden Arbeitskräftemangels in vielen Ausbildungsberufen und einem rückläufigen Erwerbspersonenpotential könnte ein solches Anwerbungsprogramm auf Ausbildungsplätze erweitert werden. Derzeit ist ein Aufenthalt zu Ausbildungszwecken laut $\S 17$ AufenthG zwar möglich, verlangt aber das Vorliegen eines Ausbildungsvertrages. Durch die Ermöglichung einer Ausbildungssuche im Inland für einen bestimmten Zeitraum könnte die Vernetzung zwischen Ausbildungsbetrieben und jungen Migranten gefördert werden, was sowohl die Suchkosten für Arbeitgeber und Migranten signifikant verringern könnte als auch die passende Besetzung offener Stellen verbessern würde (Hunger \& Krannich, 2017). Im Eckpunktpapier ist jedoch bisher nur allgemein von einer Verbesserung der Möglichkeiten des Zugangs zur Berufsausbildung die Rede (Bundesregierung, 2018). Eine verbesserte Integration in den deutschen Arbeitsmarkt könnte zudem durch sichtbare Anlaufstellen im Inund Ausland gefördert werden, die den Kontakt zwischen potentiellen Arbeitgebern und internationalen Bewerbern erleichtern (Werding, 2015). Dies soll nun in der Tat durch eine Verbesserung der Informationsmöglichkeiten für ausländische Bewerber und Bewerberinnen sowie eine Unterstützung bei Verwaltungsverfahren angestrebt werden (Bundesregierung, 2018).

Wie bereits erwähnt hat Deutschland auf Grund des sinkenden Erwerbspersonenpotentials einen Bedarf an Fachkräften unterschiedlichster Ausbildungsniveaus, weshalb nicht nur ein Interesse an der Zuwanderung hochqualifizierter Fachkräfte besteht. Das Eckpunktpapier bezieht sich hingegen explizit auf qualifizierte Fachkräfte (Bundesregierung, 2018). Auch wenn im Papier von einer ethisch verantwortbaren Gewinnung von Fachkräften die Rede ist, bleibt festzuhalten, dass eine zu starke Fokussierung auf Qualifikationen außerdem die Gefahr eines brain drains in den Her- 
kunftsregionen verstärkt. Dies sollte ebenfalls im Rahmen einer ganzheitlichen und nachhaltigen Migrationspolitik Berücksichtigung finden.

\subsection{Mögliche Synergien zwischen Asyl- und Migrationspolitik}

Eine weitere bedeutende Frage ist es, in wieweit mögliche Synergien zwischen Asyl- und Migrationspolitik denkbar sind und in der Praxis genutzt werden können. Grundsätzlich sind beide Politikbereiche voneinander zu trennen, da das Ziel der Asylpolitik die Gewährung von Schutz vor Verfolgung und Konflikten ist und somit humanitären Motiven und nicht volkswirtschaftlichen Überlegungen folgt. Angesichts der oben beschriebenen Verflechtung zwischen Asyl- und arbeitsmarktbezogener Migration sowie hoher Ablehnungsraten und einer starken Belastung des Asylsystems in den letzten Jahren stellt sich jedoch die Frage, ob mögliche Synergien zwischen beiden Bereichen denkbar sind. Auch der Deutsche Städte- und Gemeindebund kritisierte 2017 die oftmals starren deutschen Integrationsmuster und fordert eine flexiblere und schnellere Integration in den Arbeitsmarkt (Zeit, 2017, 25. Dezember).

Häufig diskutiert wird in diesem Zusammenhang ein so genannter Spurwechsel für Menschen, die als Asylbewerber nach Deutschland kommen, jedoch auch über das Migrationsrecht einen Aufenthaltstitel erhalten könnten. Dies wäre insbesondere für Asylbewerber ohne Schutzbedarf attraktiv, da sich so eine realistische Bleibeperspektive eröffnen könnte. Gelingt eine Beschäftigungsaufnahme, wäre ein Bleiberecht auch dann sinnvoll, wenn der Asylantrag abgelehnt wird oder der Fluchtgrund entfällt (Klingholz, 2015). Eine solche Verzahnung von Asyl- und Arbeitsmigrationspolitik existiert bereits seit 2008 in Schweden. Bei einer Ablehnung des Asylantrages kann eine Aufenthaltserlaubnis zum Zweck der Erwerbstätigkeit beantragt werden, falls der betroffene Asylbewerber bereits vier Monate in Schweden gearbeitet hat und eine Weiterbeschäftigung gesichert ist. Studien zufolge wird der Spurwechsel dabei nicht als Form der Arbeitsmigration, sondern als humanitäre Alternative zum Asylrecht wahrgenommen (Parusel, 2016). Das aktuelle Eckpunktpapier sieht formal keinen Spurwechsel vor und hält die Trennung von Asyl- und Migrationspolitik somit aufrecht. Allerdings will sie Kriterien für einen verlässlichen Status für Geduldete, die in den Arbeitsmarkt integriert sind schaffen, sodass diese arbei- 
ten können ohne eine Abschiebung befürchten zu müssen. Zudem soll die im Koalitionsvertrag vereinbarte Ausbildungsduldung (3+2 Regelung) einheitlich umgesetzt werden und so für mehr Planungssicherheit sorgen (Bundesregierung, 2018). Eine weitere diskutierte Verzahnung von Asylund Migrationspolitik, über die in Zukunft nachgedacht werden könnte, ist eine Integration von geographischen Prioritäten für Krisengebiete bei der Vergabe von Aufenthaltstiteln (Angenendt et al., 2017).

Es bleibt festzuhalten, dass Flüchtlingen derzeit die Integration in die Aufnahmegesellschaft im Vergleich zu Arbeitsmigranten deutlich erschwert wird. Dies drückt sich in Deutschland trotz eines rechtlichen $\mathrm{Zu}$ gangs zum Arbeitsmarkt für Schutzbedürftige und verschiedener Unterstützungsmaßnahmen unter anderem in einer geringeren Erwerbstätigkeitsrate aus (EMN synthesis report, 2015). ${ }^{30}$ Da sie ihr Land nicht freiwillig verlassen, sind Flüchtlinge konzeptionell in vielfacher Hinsicht benachteiligt (Richmond, 1988). Neben eventuellen physischen und mentalen Traumata haben Flüchtlinge in der Regel keine bis wenige Möglichkeiten sich auf die Aufnahmegesellschaft vorzubereiten und so ihre Integrationschancen durch Sprach- und Qualifikationserwerbe zu verbessern (Brücker et al., 2015). Zudem ist der Berufs-Ausbildungs-Mismatch bei Asylbewerbern deutlich größer als bei anderen Zuwanderungsgruppen. Dies liegt auch an der erschwerten Anerkennung von Ausbildungen aufgrund fehlender Unterlagen (Bock-Schappelwein \& Huber, 2015). Aus diesem Grund wären Unterstützungen bei der Anerkennung ausländischer Qualifikationen sowie Politikmaßnahmen, die eine intensivere Förderung des Erwerbs von Sprach- und Berufsqualifikationen direkt nach der Einreise ermöglichen entscheidend, um die Integration von Flüchtlingen zu verbessern. Eine große Verantwortung fällt hier Schulen und Universitäten zu, die einen maßgeblichen Beitrag zur Integration leisten können (Brücker et al., 2015).

Ein zentrales Problem für Flüchtlinge, das durch die Möglichkeit eines Spurwechsels gelöst werden könnte, sind Restriktionen bei der Arbeitssuche. So besitzen Flüchtlinge und Asylbewerber in Deutschland die ersten drei Monate wenn sie sich in einer Erstaufnahmeeinrichtung befinden kei-

30 In der Literatur wird in diesem Zusammenhang von der refugee gap gesprochen (siehe z.B. Connor, 2010). 
nerlei Zugang zum Arbeitsmarkt oder Integrationskursen. ${ }^{31}$ Wann ein eingeschränkter (bei Ablehnung des Asylantrages) beziehungsweise unbeschränkter Zugang (bei Gewährung des Schutzstatus) jedoch tatsächlich erteilt wird hängt maßgeblich von der Bearbeitungsgeschwindigkeit der Anträge ab (Brücker et al., 2015). ${ }^{32}$ Diese Wartezeit verursacht nicht nur dem Staat erhebliche Kosten, sondern besonders auch den Flüchtlingen, da die Diskontinuität im Bildungs- und Arbeitsleben somit verlängert wird. Ein großes Problem ist zudem die geringe Rechtssicherheit während des Verfahrens, die es für Arbeitgeber riskant macht Asylbewerber zu beschäftigen. Ein wesentlicher Vorteil eines Spurwechsels wäre somit die Ermöglichung einer vom Asylgrund unabhängigen Planungssicherheit bezüglich der Bleibedauer und des Aufenthaltsstatus. Auch wenn sich das Problem durch einen verlässlichen Status für Geduldete mit Arbeit und die Ausbildungsduldung verringern dürfte bleibt hier Handlungsbedarf. Für die Asylbewerber selbst kann die aktuelle Unsicherheit neben psychologischen Kosten auch zu geringeren Investitionsanreizen in länderspezifisches Humankapital, wie den Spracherwerb führen. Neben humanitären Gründen wäre daher auch aus ökonomischer Sicht eine Verkürzung der Asylverfahren und ein schneller Zugang zum Arbeitsmarkt essentiell. ${ }^{33}$ Laut Schätzungen des IAB (2016) existierten Ende 2015 etwa 154.000 offene Arbeitsstellen, die auch für Flüchtlinge ohne Berufsausbildung relevant wären. Initiativen von IHK und HWK, die Ausbildungsbetriebe und Flüchtlinge zusammenbringen, stellen in diesem Zusammenhang wertvolle Beiträge zur Integration dar und zeigen die Chancen, die sich für beide Seiten ergeben (siehe z.B. IHK, 2018).

31 Seit 2015 ist gemäß $§ 61$ AsylG die Aufnahme einer Beschäftigung für Asylbewerber nach drei Monaten unter bestimmten Voraussetzungen möglich.

322016 lag die durchschnittliche Bearbeitungszeit von Asylanträgen bei rund 7 Monate. Nach einem Anstieg auf durchschnittlich 10,7 Monate im Jahr 2017 liegt die Bearbeitungsdauer im zweiten Quartal 2018 wieder im Schnitt bei 7,3 Monaten (Deutscher Bundestag, 2018).

33 Dabei darf eine Verkürzung selbstverständlich nicht zu einer Minderung des verfahrensrechtlichen Schutzstandards führen. 


\section{ZUSAMMENFASSUNG UND SCHLUSSBEMERKUNGEN}

Es bleibt festzuhalten, dass die oft negative öffentliche Wahrnehmung von Zuwanderung nicht mit der volkswirtschaftlichen Realität übereinstimmt. Es ist daher die Aufgabe von Politik und Medien über die ökonomischen Konsequenzen von Zuwanderung aufzuklären. Studien zeigen, dass langfristig negative Lohn- und Beschäftigungswirkungen, wenn überhaupt, gering ausfallen und im Falle eines hohen Anteils an hochqualifizierten Arbeitskräften sogar positive Auswirkungen zu erwarten sind. Aus fiskalischer Sicht scheint Immigration in den meisten OECD Ländern einen positiven, aber geringen Effekt zu haben. Aufgrund des demographischen Wandels müssen in den nächsten Jahren zudem eine Reihe an Maßnahmen zur Steigerung des Erwerbspersonenpotentials ergriffen werden. Die mit der Alterung der Bevölkerung verbundenen Probleme können durch eine vermehrte Zuwanderung zwar nicht gelöst, jedoch verringert werden. Da in einer wissensbasierten Gesellschaft die Bedeutung des Faktors Humankapital zudem weiterhin steigen wird, steht Deutschland in einem zunehmenden Wettbewerb um internationale Fachkräfte.

In diesem Kontext kann ein Einwanderungsgesetz eine positive Signalwirkung ausüben, die Attraktivität Deutschlands als Einwanderungsland erhöhen sowie institutionelle Hürden bei der Zuwanderung weiter abbauen. Das Eckpunktpapier ist ein erster Schritt in diese Richtung. Um von den positiven Konsequenzen von Migration profitieren zu können ist zudem eine schnelle Integration in den deutschen Arbeitsmarkt von zentraler Bedeutung. Aus diesem Grund sollte besonders der Kontakt zwischen Arbeitgebern und Migranten verbessert werden, mit einem besonderen Fokus auf die Anwerbung von Auszubildenden und Studierenden. Auch könnten potentielle Synergien zwischen Asyl- und Migrationspolitik genutzt werden, die eine Entflechtung von Wanderungsmotiven erleichtern. Auffallend ist, dass Flüchtlinge vor allem in den Anfangsjahren hinter Arbeitsmigranten zurückfallen. Aus diesem Grund ist es von großer Bedeutung, dass der Staat die Integration durch Investitionen in sprachliche und berufliche Qualifikationen, aber auch durch einen schnellen Abbau von Unsicherheiten fördert. Schließlich sollte im Rahmen der Migrationspolitik auch eine entwicklungspolitische Verantwortung berücksichtigt werden und die Gefahr eines brain drain in den Herkunftsstaaten ernst genommen werden. Eine 
mögliche Verbindung von Migrations- und Entwicklungspolitik könnte dabei beispielsweise durch eine Förderung temporärer und zirkulärer Migrationsformen erreicht werden.

\section{LITERATUR}

Aiyar, S., Barkbu, B., Batini, N., Berger, H., Detragiache, E., Dizioli, A., Ebeke, C., Lin, H., Kaltani, L., Sosa, S., \& Spilimbergo, A. (2016). The Refugee Surge in Europe. Europe: Imfstaff Discussion Note (SDN/ $16 / 02)$.

Angenendt, S. (2012). Migration, Mobilität und Entwicklung. EUMobilitätspartnerschaften als Instrument der Entwicklungszusammenarbeit. SWP-Studie. Stiftung Wissenschaft und Politik Deutsches Institut für Internationale Politik und Sicherheit, Berlin.

Angenendt, S., Kipp, D., \& Meier, A. (2017). Gemischte Wanderungen. Herausforderungen und Optionen einer Dauerbaustelle der deutschen und europäischen Asyl- und Migrationspolitik. Bertelsmann Stiftung, Gütersloh.

Bader, N., \& Steffen, T. (2018). Einwanderungsgesetz - Mehr Chancen für ausländische Fachkräfte. Zeit online. Online verfügbar unter: https: //www.zeit.de/politik/deutschland/2018-10/einwanderungsgesetz-auslae ndische-fachkraefte-zuwanderung-faq\#worauf-haben-sich-die-koalition spartner-geeinigt.

BAMF (2018). Aktuelle Zahlen zu Asyl. Ausgabe: Oktober 2018. Online verfügbar unter: http://www.bamf.de/SharedDocs/Anlagen/DE/Down loads/Infothek/Statistik/Asyl/aktuelle-zahlen-zu-asyl-oktober-2018.pdf ?__blob=publicationFile.

Beine, M., Docquier, F., \& Rapoport, H. (2001). Brain drain and economic growth: theory and evidence. Journal of Development Economics, 64(1), 275-289.

Beine, M., Docquier, F., \& Rapoport, H. (2008). Brain Drain and Human Capital Formation in Developing Countries: Winners and Losers. The Economic Journal, 118, 631-652.

Bermingham, J. R. (2001). Immigration: Not a solution to problems of population decline and aging. Population \& Environment, 22(4), 355363. 
Bhagwati, J., \& Rodriguez, C. (1975). Welfare-theoretical analyses of the brain drain. Journal of Development Economics, 2(3), 195-221.

Bishop, M. (2004). Essential Economics. The Economist (book 67). Bloomberg Press.

Blau, F. D. (1984). The use of transfer payments by immigrants. Industrial \& Labor Relations Review, 37(2), 222-239.

Bock-Schappelwein, J., \& Huber, P. (2015). Auswirkungen einer Erleichterung des Arbeitsmarktzuganges für Asylsuchende in Österreich. Österreichisches Institut für Wirtschaftsforschung, WIFO project, (9714).

Boeri, T. (Ed.), Brücker, H., Docquier, F., \& Rapoport, H. (2012). Brain drain and brain gain: The global competition to attract high-skilled migrants. Oxford: Oxford University Press.

Bonin, H. (2002). Eine fiskalische Gesamtbilanz der Zuwanderung nach Deutschland. Vierteljahrshefte zur Wirtschaftsforschung, 71(2), 215229.

Borjas, G. J., \& Trejo, S. (1991) Immigrant participation in the welfare system. Industrial and Labor Relations Review, 44(2), 195-211.

Borjas, G. J. (2003). The labor demand curve is downward sloping: Reexamining the impact of immigration on the labor market. The Quarterly Journal of Economics, 118(4), 1335-1374.

Brücker, H., Hauptmann, A., \& Trübswetter, P. (2015). Asyl-und Flüchtlingsmigration in die EU und nach Deutschland. IAB-Aktuelle Berichte, 8, 2015.

Brücker, H. et al. (2016). IAB-BAMF-SOEP-Befragung von Geflüchteten. Flucht, Ankunft in Deutschland und erste Schritte der Integration, Ausgabe 5|2016 der Kurzanalysen des Forschungszentrums Migration, Integration und Asyl des Bundesamtes für Migration und Flüchtlinge, Nürnberg.

Brücker, H., \& Jahn, E. J. (2011). Migration and Wage-setting: Reassessing the Labor Market Effects of Migration. The Scandinavian Journal of Economics, 113(2), 286-317.

Büchel F., \& Frick J. (2005). Immigrants' economic performance across Europe - Does immigration policy matter?, Population Research and Policy Review, 24(2), 175-212.

Bundesregierung (2018). Eckpunkte zur Fachkräfteeinwanderung aus Drittstaaten. Online verfügbar unter: https://www.bmi.bund.de/Shared 
Docs/downloads/DE/veroeffentlichungen/2018/eckpunkte-fachkraefteei nwanderung.pdf? _ blob=publicationFile \&v=1.

Chiswick, B.R. (2005). High Skilled Immigration in the International Arena. IZA Discussion Paper, No.1782. Bonn.

Connor, P. (2010). Explaining the refugee gap: Economic outcomes of refugees versus other immigrants. Journal of Refugee Studies, 23(3), 377397.

D'Amuri, F., Ottaviano, G. I., \& Peri, G. (2010). The labor market impact of immigration in Western Germany in the 1990s. European Economic Review, 54(4), 550-570.

Dancygier, R. M., \& Laitin, D. D. (2014). Immigration into Europe: Economic discrimination, violence, and public policy. Annual Review of Political Science, 17, 43-64.

Demary, M., \& Erdmann, V. (2012). Fachkräfteengpässe und Arbeitslosigkeit in Europa: Wanderung als kurzfristiger Ausgleichmechanismus. IW-Trends-Vierteljahresschrift zur empirischen Wirtschaftsforschung, 39(3), 35-48.

Deutscher Bundestag (2018). Ergänzende Informationen zur Asylstatistik für das erste und zweite Quartal 2018 - Schwerpunktfragen zur Asylverfahrensdauer. Online verfügbar unter: http://dip21.bundestag.de/ $\operatorname{dip} 21 /$ btd/19/038/1903861.pdf.

Docquier, F. (2013). Cross-Border Migration, Employment and Economic Growth. Background Research Paper, FNRS and IRES.

Docquier, F., Özden, C., \& Peri, G. (2014). The labour market effects of immigration and emigration in OECD countries. The Economic Journal, 124(579), 1106-1145.

Docquier, F., \& Rapoport, H. (2012). Globalization, Brain Drain, and Development. Journal of Economic Literature, 50 (3), 681-730.

Dumont, J.C., \& Liebig, T. (2014). Is migration good for the economy? Migration Policy Debates, OECD, May 2014.

EMN inform (2015). Determining labour shortages and the need for migration labour from third countries in the EU. Online verfügbar unter: https:/ec.europa.eu/home-affairs/sites/homeaffairs/files/what-we-do/net works/european_migration_network/reports/docs/emn-informs/inform_ labour_shortages_nov_2015_final.pdf.

EMN synthesis report (2015). Integration of beneficiaries of international/humanitarian protection into the labour market: policies and good practices. Online verfügbar unter: http://www.bamf.de/SharedDocs/An 
lagen/DE/Publikationen/EMN/SyntheseberichteInform/ZuStudien/emnwp66-synthese-unterstuetzung-schutzberechtigte-arbeitsmarkt.html.

Ette, A., Rühl, S., \& Sauer, L. (2012). Die Entwicklung der Zuwanderung hochqualifizierter Drittstaatsangehöriger nach Deutschland. Zeitschrift für Ausländerrecht und Ausländerpolitik, 32 (1-2), 14-20.

Eurostat (2018). Asylum statistics. Online verfügbar unter: http://ec.eu ropa.eu/eurostat/statistics-explained/index.php/Asylum_statistics.

Eurostat (2018). Residence permits statistics. Online verfügbar unter: http://appsso.eurostat.ec.europa.eu/nui/show.do?dataset=migr_resbc $1 \& 1$ ang=de.

Felbermayr, G.J., \& Reczkowski, I. (2012). International student mobility and high-skilled migration: the evidence. Ifo Working Paper, No.132. München.

Findlay, A. M., \& Wahba, J. (2013). Migration and demographic change. Population, Space and Place, 19(6), 651-656.

Geiger, M., \& Steinbrink, M. (2012). Migration und Entwicklung: Merging Fields in Geography. IMIS-Beiträge, 42, 7-36, Osnabrück.

Gibson, J., \& McKenzie, D. (2010). The economic consequences of Brain Drain of the best and brightest: Microeconomic evidence from five countries. Discussion Paper Series, Forschungsinstitut zur Zukunft der Arbeit, No. 5124.

Grubel, H. B., \& Scott, A. D. (1966). The international flow of human capital. The American Economic Review, 56(1/2), 268-274.

Hinte, H., Rinne, U., \& Zimmermann, K. F. (2011). Ein Punktesystem zur bedarfsorientierten Steuerung der Zuwanderung nach Deutschland. Gutachten im Auftrag des sächsischen Staatsministeriums für Wirtschaft, Arbeit und Verkehr, Bonn.

Hunger, U. (2003). Vom Brain Drain zum Brain Gain. Migration, Netzwerkbildung und sozioökonomische Entwicklung: das Beispiel der indischen "Software-Migranten". IMIS-Beiträge, 16, 7-22, Osnabrück.

Hunger, U., \& Krannich, S. (2017). Einwanderung neu gestalten - transparent, attraktiv, einfach. WISO Diskurs 05/2017, Gesprächskreises Migration und Integration in der Abteilung Wirtschafts- und Sozialpolitik der Friedrich-Ebert-Stiftung.

IAB (Institut für Arbeitsmarkt- und Berufsforschung) (2016). Schätzung der Zahl der für Flüchtlinge relevanten Arbeitsstellen. Aktuelle Berichte 
12/2016, Nürnberg. Online verfügbar unter: http://doku.iab.de/aktuell/ 2016/aktueller_bericht_1612.pdf.

IHK (2018). IHK-Aktionsprogramm „Ankommen in Deutschland - Gemeinsam unterstützen wir Integration. " Online verfügbar unter: http:// www.dihk.de/themenfelder/wirtschaftspolitik/fachkraeftesicherung-ver antwortung/integration/integration-fluechtlinge.

IOM (International Organisation for Migration) (2008). World Migration 2008: Managing Labour Mobility in the Evolving Global Economy. Geneva: International Organisation for Migration.

Kancs, D., \& Lecca, P. (2017). Long-term Social, Economic and Fiscal Effects of Immigration into the EU: The Role of the Integration Policy. JRC Working Papers in Economics and Finance, 2017/4.

Kermer, S. (2007). Verstädterung, Migration und wirtschaftliche Entwicklung, Vol. 5. Münster: LIT Verlag.

Kerr, S. P., \& Kerr, W. R. (2011). Economic impacts of immigration: A survey. No. w16736. National Bureau of Economic Research.

Klingholz, R. (2015). Einwanderungsland Deutschland: Wie sollte die Zuwanderung beeinflusst werden?. Ifo Schnelldienst, 68(03), 5-22.

Kingma, M. (2018). Nurses on the move: Migration and the global health care economy. Cornell University Press.

Langenfeld, C. (2015). Einwanderungsland Deutschland: Wie sollte die Zuwanderung beeinflusst werden?. Ifo Schnelldienst, 68(03), 5-22.

Langthaler, M., \& Hornoff, S. (2008). Braindrain und seine Auswirkungen auf Entwicklungsländer. Working Paper, No. 20, Österreichische Forschungsstiftung für Internationale Entwicklung, Wien.

Lipsmeyer, C. S., \& Zhu, L. (2011). Immigration, globalization, and unemployment benefits in developed EU states. American Journal of Political Science, 55(3), 647-664.

Martin, P. (2004). Migration. In Bjorn Lomberg (Ed.) Global crises, global solutions, Cambridge University Press.

Mattoo, A., Neagu, I.C., \& Özden, C. (2008). Brain waste? Educated immigrants in the US labor market. Journal of Development Economics, 87 (2), 255-269.

Mishan, E. J., \& Needleman, L. (1968). Immigration: some long term economic consequences. Economia Internazionale, 21 (2), 281-300.

Mountford, A. (1997). Can a brain drain be good for growth in the source economy?. Journal of Development Economics, 53(2), 287-303. 
Okkerse, L. (2008). How to measure labour market effects of immigration: A review. Journal of Economic Surveys, 22(1), 1-30.

Oltmer, J. (2012). Globale Migration - Geschichte und Gegenwart. Vol. 2761, München: CH Beck.

Ottaviano, G. I., \& Peri, G. (2012). Rethinking the effect of immigration on wages. Journal of the European Economic Association, 10(1), 152-197.

Özden, C., \& Schiff, M. (2006). International migration, remittances and the brain drain. Washington DC: World Bank.

Parusel, B. (2016). Spurwechsel und zirkuläre Migration in Schweden Ansätze eines flexiblen Migrationsrechts? In Barwig, K., BeichelBenedetti, S., \& Brinkmann, G. (Hrsg.): Gerechtigkeit in der Migrationsgesellschaft (pp.257-280). Hohenheimer Tage zum Ausländerrecht 2015. Baden-Baden: Nomos.

Piyapromdee, S. (2014). The impact of immigration on wages, internal migration and welfare. Working paper.

Pries, L. (2001): Internationale Migration. Einsichten. Themen der Soziologie. Bielefeld: transcript Verlag.

Ratha, D., Eigen-Zucchi, C., \& Plaza, S. (2016). Migration and remittances Factbook 2016. World Bank Publications.

Richmond, A. H. (1988). Sociological theories of international migration: the case of refugees. Current Sociology, 36(2), 7-25.

Schaland, A.J. (2012). Wissen wandert: die Bedeutung von Remigranten für die wissensbasierte Regionalentwicklung in Entwicklungs- und Schwellenländern. IMIS Beiträge, 42, 113-130, Osnabrück.

Schneider, J., \& Parusel, B. (2011). Zirkuläre und temporäre Migration. Empirische Erkenntnisse, politische Praxis und zukünftige Optionen in Deutschland. Studie der Deutschen Nationalen Kontaktstelle für das Europäische Migrationsnetzwerk (EMN). Bundesamt für Migration und Flüchtlinge.

Simon, S. (2011). Europaweite Rekrutierung von Fachkräften. Zentrum für wirtschaftspolitische Forschung (ZWF), HTW Chur.

Sinyolo, D. (2012), A strategy for managing teacher migration in Southern Africa, University of South Africa, Pretoria.

Statistisches Bundesamt (2015). Bevölkerung Deutschlands bis 2060 - 13. Koordinierte Bevölkerungsvorausberechnung. Online verfügbar unter: hattps://www.destatis.de/DE/ZahlenFakten/GesellschaftStaat/Bevoelker ung/Bevoelkerungsvorausberechnung/Bevoelkerungsvorausberechnung .html. 
Straubhaar, T. (2015). Wieso ein Einwanderungsgesetz?. Wirtschaftsdienst, 95(8), 510-511.

Von Hausen, N. (2010). Teufelskreis im Ankunftsland: Zur Verstetigung hochqualifizierter MigrantInnen im Arbeitsmarkt für unspezifische Qualifikationen. In Nohl, A., Schittenhelm, K. Oliver Schmidtke, O. \& Weiß, A. (Hrsg.): Kulturelles Kapital in der Migration, 180-194, VS Verlag für Sozialwissenschaften.

Waldrauch, H. (1995). Theorien zu Migration und Migrationspolitik. Journal für Sozialforschung, 1, 27-49.

Werding, M. (2015). Einwanderungsland Deutschland: Wie sollte die $\mathrm{Zu}-$ wanderung beeinflusst werden?. Ifo Schnelldienst, 68(03), 5-22.

Wrobel, R. (2016). Der deutsche Arbeitsmarkt zwischen Fachkräftemangel und Immigration: Ordnungspolitische Perspektiven in der Flüchtlingskrise. Ordnungspolitische Diskurse, 2016-01.

Zeit (2017, 25. Dezember). Kommunen warnen vor Scheitern der Flüchtlingsintegration. Zeit Online. Verfügbar unter: http://www.zeit.de/poli tik/deutschland/2017-12/integration-fluechtlinge-arbeitsmarkt-kommun en. 



\title{
Der Bedarf nach einem neuen Einwanderungsrecht
}

\author{
Eine Analyse aus juristischer Perspektive
}

Nils Imgarten

In Anbetracht des erneuten starken Anstiegs an Zuwanderung nach Deutschland und Europa seit etwa $2012^{1}$ hat das Migrationsrecht wieder zunehmend gesellschaftlich wie auch politisch Beachtung gefunden. Geführt hat dies zu zahlreichen Gesetzesnovellierungen auf europäischer wie nationaler Ebene, insbesondere im Asylrecht. ${ }^{2}$ Begleitet wird dieser Prozess stets von einer teils hitzig geführten Debatte zum Bedarf nach einem neuen Einwanderungsrecht, ${ }^{3}$ bei welcher regelmäßig Begriffe wie Flucht, Migration oder Einwanderung durcheinander geraten oder synonym verwendet werden. ${ }^{4}$

Dieser Beitrag soll helfen, begrifflich wie inhaltlich Klarheit über die Gemeinsamkeiten und Unterschiede von Flucht und Einwanderung und das Verhältnis zueinander zu schaffen und dabei einen Überblick über das $\mathrm{Zu}$ -

1 Vgl. Eurostat, Aufenthaltstitelstatistik.

2 Vgl. zum Überblick: Wissenschaftlicher Dienst des Deutschen Bundestages, Änderungen des Asyl- und Aufenthaltsrechts seit Januar 2015.

3 In jüngerer Vergangenheit wurde diese erneut angestoßen durch die Bundestagsfraktion von B90/Die Grünen, vgl. BT-Drs. 18/3915 und dazu Plenarprotokoll 18/85, S. 8029ff.

4 Exemplarisch aber keinesfalls alleinstehend Wergin, in Die WELT vom 5.9.2015. 
sammenwirken von Asyl- und Migrationsrecht geben. Zentraler Ausgangspunkt der Betrachtung ist das geltende Recht, insbesondere das Aufenthaltsgesetz ${ }^{5}$ (dazu Kapitel 3.2). Im Weiteren soll jedoch auch de lege feren$d a$ der häufig geltend gemachte Reformbedarf ${ }^{6}$ unter Berücksichtigung der jüngeren Gesetzgebungsvorschläge der Bundestagsfraktionen von $S P D$ und B90/Die Grünen ${ }^{7}$ und des Rates der Europäischen Union ${ }^{8} \mathrm{zu}$ einem neuen Einwanderungsrecht beurteilt werden (dazu Kapitel 5.2). Die Ankündigungen eines Gesetzgebungsentwurfs für ein Fachkräfteeinwanderungsgesetz durch die Bundesregierung im Oktober 2018 sollen hierbei punktuell vergleichend in die Analyse einbezogen werden, wobei sich bereits nach dem Stand dieses Beitrags vom November 2018 eine inhaltliche Teilkongruenz mit früheren Gesetzgebungsinitiativen und eigenen Vorschlägen abzeichnet.

\section{DIE VERSTRICKUNG ZWISCHEN ASYL- UND EINWANDERUNGSRECHT}

Es wurde diskutiert, ob das bestehende Asylsystem bei einer hohen Fallzahl wie etwa im Jahr 2015 an seine Grenzen gerät und dem eigenen Anspruch einer differenzierten Einzelfallprüfung, vgl. § 24 I Asylgesetz (AsylG), nicht gerecht werden kann. ${ }^{9}$ Zugleich zeigt sich, dass sich unter den Asylantragstellenden regelmäßig Personen befinden, für die es in der EU kaum

5 Gesetz über den Aufenthalt, die Erwerbstätigkeit und die Integration von Ausländern im Bundesgebiet in der Fassung vom 25.2.2008, zuletzt geändert am 12.7.2018 (BGB1. I S. 1147).

6 S. nur $B D A$, Stellungnahme vom 26.4.2017.

7 SPD-Bundestagsfraktion, Entwurf eines Einwanderungsgesetzes (EinwG-E) v. 7.11.2016, eingebracht in den Deutschen Bundestag am 8.11.2017, vgl. Bt-Drs. 19/44; B90/Die Grünen, EinwG-E, BT-Drs. 18/11854.

8 Vorschlag für eine Richtlinie des europäischen Parlaments und des Rates COM(2016) 378.

$9 \mathrm{Zu}$ den Zahlen vgl. BAMF, Aktuelle Zahlen zu Asyl 08/2017, S. 12; zum Diskurs vergleiche den Beitrag von Merve Kania in diesem Band. 
Aussicht auf Gewährung von internationalem Schutz gibt. ${ }^{10}$ Meist ist der Grund hierfür, dass diese Personen aus ökonomischen oder ökologischen Gründen migrieren und somit keinen Schutz nach der Genfer Flüchtlingskonvention $^{11}$ genießen, welche gemäß Art. 1 lit. A. Nr. 2 exklusiv als Migrationsgründe die begründete Furcht vor Verfolgung wegen der „Rasse“, Nationalität, Religion, politischen Überzeugung oder Zugehörigkeit zu einer bestimmten sozialen Gruppe vorsieht. Andere Motive der Migration sollen gerade keine Berücksichtigung finden. Diese Unterscheidung des internationalen Rechts wird in $\S 3$ I Nr. 1 AsylG ins nationale Recht übernommen und um den europarechtlich vorgegebenen Titel des subsidiären Schutzes in $§ 4$ AsylG ergänzt, was jedoch zu keiner geänderten Bewertung führt.

Im Ergebnis leidet die effektive Rechtsanwendung im Asylrecht also darunter, dass die Verfahren häufig für Personen durchgeführt werden, für die das Asylrecht eigentlich gar nicht vorgesehen ist.

Eine Reaktion auf diese ungewollte Migration war die verstärkte Abschottung an den Außengrenzen der Europäischen Union (EU), welche zunächst zu Grenzschließungen und der Errichtung von Zäunen auf dem Landweg der bis dahin von vielen Migranten gewählten sogenannten „Balkan-Route“ führte. ${ }^{12}$ In Folge dessen kam es zu einer stärkeren Nutzung der gefährlichen Migrationsroute über das zentrale Mittelmeer. Allein 2016 starben 5143 Menschen bei der Überquerung des Mittelmeers. Zu beobachten ist zudem ein Anstieg der Todesrate von 1,2\% 2016 auf 2,1\% in der ersten Jahreshälfte 2017. ${ }^{13}$ Vom Standpunkt des internationalen Seerechts begegnet die bisherige Strategie der Abschottung an den Außengrenzen bereits erheblichen Bedenken. ${ }^{14}$ Darüber hinaus bleibt festzustellen, dass sie auch nur begrenzt Wirkung zeigt.

10 Das ergeben die Ablehnungsquoten. Vgl. exemplarisch BAMF, Aktuelle Zahlen zu Asyl 08/2017, S. 10.

11 Abkommen über die Rechtsstellung der Flüchtlinge vom 28.7.1951.

12 Pro Asyl, Balkanroute: Eine Chronik der Abschottung.

13 Es bestehen Schwierigkeiten bei der exakten Erfassung und Verifikation der Zahlen. Diese Werte basieren auf IOM, Fatal Journeys, Vol. 3, S. 6.

14 Siehe den Beitrag von Maximilian Pichl und Timo Tohidipur in diesem Band. 
Es gibt weiterhin eine hohe Anzahl an Migrierenden, welche so starke Migrationsgründe (sog. push-factors) haben, ihr Land zu verlassen, dass sie sogar die hohen Risiken einer Mittelmeerüberquerung auf sich nehmen, nur für eine vage Chance, eine Zukunft in Europa zu finden. ${ }^{15}$

In konsequenter Fortführung der Abschottungspolitik wird nun über die Vorverlagerung der europäischen Grenzpolitik und Grenzkontrollen in Drittstaaten diskutiert. ${ }^{16}$

Unter der Prämisse, dass es Aufgabe und Verantwortung der deutschen und europäischen Entscheidungsträger sei, Lösungen für die prekäre Lage im Mittelmeer zu entwickeln erfolgt die folgende Analyse des Asyl- und Einwanderungsrechts insbesondere mit Bezugnahme auf die Gruppe von Menschen, welche (versuchen) über das Mittelmeer nach Europa zu gelangen. ${ }^{17}$ Der Fokus liegt dabei weniger auf dem bisher beschrittenen Weg der Abschottungspolitik, sondern auf der Diskussion von Alternativvorschlägen im Bereich der Schaffung legaler Zugangswege.

\section{DIE UNTERSCHEIDUNG ZWISCHEN REGULÄRER UND IRREGULÄRER MIGRATION ALS NORMATIVE LEITFIGUR}

Grundlegend für eine Analyse der Migrationskontrolle ist die Unterscheidung zwischen regulärer und irregulärer Migration. Diese soll am Beispiel des deutschen Migrationsrechts veranschaulicht werden, indem dieses für den hypothetischen Fall eines Migranten $M$ betrachtet wird, welcher beabsichtigt aus einem EU-Drittstaat aufgrund ökonomischer Perspektivlosigkeit nach Deutschland zu migrieren. Unterstellt wird, dass es sich um eine Person ohne Hochschulabschluss, ohne signifikante finanzielle Mittel und ohne bestehendes Angebot eines Arbeitsplatzes in der EU handelt. Begut-

15 Auch 2017 sind die Zahlen der Todesfälle im Mittelmeer trotz Schwankungen weiterhin hoch, vgl. IOM, aaO.

16 Vgl. exemplarisch für Libyen, Kopp, Externalisierung der Verantwortung.

17 Bei der Betrachtung einer gesamten Gruppe von Personen ist es selbstverständlich, dass gewisse Pauschalisierungen nicht zu vermeiden sind. Dies wird hingenommen, um die Betrachtung der Gesamtsituation zu ermöglichen. 
achtet werden hier die Möglichkeiten dieser Person, einen Aufenthaltstitel in Deutschland zu erlangen. Die systematische Darstellung hat hierbei keinen Anspruch auf Vollständigkeit der Abbildung aller erdenklichen Aufenthaltstitel, sondern konzentriert sich auf die in der Praxis relevantesten Fälle. ${ }^{18}$ Ziel der Darstellung ist vielmehr das Herausstellen der grundsätzlichen Herangehensweise des Aufenthaltsgesetzes und der Unterscheidung zwischen regulärer und irregulärer Migration.

Hinsichtlich dieser Kategorisierung bestehen bisher keine gefestigten, anerkannten Definitionen. ${ }^{19}$ Das BAMF setzt etwa irreguläre Migration mit illegaler Migration gleich. ${ }^{20}$ Die OECD wiederum verwendet den Begriff unerlaubter Migration. ${ }^{21}$ Im Folgenden wird eine eigene Begriffsabgrenzung vorgenommen.

\subsection{Begriffsklärung}

Migration dient als Oberbegriff jeglicher Wanderungsbewegungen. Abgesehen von der hier ausgeklammerten Binnenmigration kann jede Wanderungsform lückenlos kategorisiert werden in reguläre oder irreguläre Migration. Regulär ist die Migration, wenn sie nach staatlich festgelegten Abläufen und Strukturen kontrolliert abläuft. Bereits vor dem Grenzübertritt besteht ein (jedenfalls vorübergehender) Aufenthaltstitel des Zielstaats. Beispiele sind Visa, etwa zur Arbeitsmigration bzw. Arbeitsplatzsuche oder zum Studium.

Irreguläre Migration hingegen beschreibt eine Form von Migration, bei der zum Zeitpunkt des Grenzübertritts noch kein Aufenthaltstitel im Zielstaat vorhanden ist. Man könnte insoweit also auch von Migration ohne vorherige Erlaubnis (,unauthorized“, s.o.) sprechen. Beispiel hierfür ist die Migration, welche täglich über das Mittelmeer stattfindet, unabhängig da-

18 Nicht betrachtet werden dabei Spezialregelungen, welche die Zugehörigkeit zu einer für diesen Vergleich unerheblichen Gruppe voraussetzen wie etwa $\S \S 19$ b-d (Arbeitnehmer internationaler Unternehmen), $\S \S 20 \mathrm{f}$. (Forscher und Selbständige), §§ 27ff. AufenthG (Familiennachzug).

19 Vgl. Zum Diskussionsstand Angenendt, Irreguläre Migration als Problem, S. 10.

20 So aber der Migrationsbericht der Bundesregierung (2015), S. 151.

21 Diese Formulierung findet sich vereinzelt etwa in Veröffentlichungen der OECD, vgl. OECD, International Migration Outlook, Annual Report (2007). 
von, ob aus Gründen individueller Verfolgung oder aus ökonomischer Motivation. Der Begriff der illegalen Migration hingegen erscheint nicht adäquat insoweit, dass er an eine rechtliche Bewertung anknüpft, die jedoch nicht zum Zeitpunkt der Migration, sondern erst nach Abschluss eines Verwaltungsverfahrens, beispielsweise eines Asylverfahrens, im Zielland möglich ist.

\subsection{Reguläre Migration nach dem Aufenthaltsgesetz}

\subsubsection{Aufenthalt zum Zweck der Ausbildung}

Ein Aufenthalt für die Dauer eines Ausbildungsabschnitts ist grundsätzlich möglich nach den $\S \S 16 f f$. Aufenthaltsgesetz (AufenthG). Jedoch bestehen zwei wesentliche Gründe, weshalb dieser Aufenthalt für $M$ nicht möglich ist. Einerseits wird nach $\S 16$ I 1 AufenthG vorausgesetzt, dass bereits eine Zulassung der Bildungseinrichtung vorliegen muss. Dies setzt regelmäßig eine deutsche Hochschulzugangsberechtigung oder einen vergleichbaren Abschluss und außerdem hinreichende Sprachkenntnisse in der Unterrichtssprache voraus.

Zwar kann grundsätzlich auch nach $\S 16$ VII AufenthG zum Zweck der Studienbewerbung eine Aufenthaltserlaubnis für bis zu neun Monate erteilt werden. Jedoch zeigt dies bereits eine Ausrichtung des $\S 16$ AufenthG auf Länder, welche ein mit der EU vergleichbares Bildungsniveau haben. Dies wird bestätigt durch das Erfordernis der Sicherung des eigenen Lebensunterhalts, vgl. § 5 I Nr. 1 AufenthG, welcher in der Ausbildungsphase mindestens ein Niveau in Höhe des BaFöG-Höchstsatzes fordert ${ }^{22}$ und daher für Personen aus weniger wohlhabenden Gesellschaften eine weitere Hürde darstellt.

\subsubsection{Aufenthalt zum Zweck der Beschäftigung}

Im gegebenen häufigen Fall, dass $M$ aufgrund ökonomischer Perspektivlosigkeit im Herkunftsstaat migriert kann man annehmen, er suche nach einer Beschäftigung. Grundsätzlich ist es nach $\S \S 18,19$, 19a AufenthG möglich im Falle einer Beschäftigung einen Aufenthaltstitel zu erteilen.

Gemäß $\S 18$ V Alt. 1 AufenthG ist diese Option jedoch begrenzt auf Fälle, in denen bereits ein konkretes Arbeitsplatzangebot nachgewiesen

22 Christ, in: Kluth/Heusch, Ausländerrecht, § 16 Rn. 1. 
werden kann. ${ }^{23}$ Teils wird sogar der Nachweis nach dem tatsächlichen Bestehen des Beschäftigungsverhältnisses gefordert. ${ }^{24}$ Jedoch auch der bloße Nachweis eines Angebots wird für Migranten aus ihrem Herkunftsstaat heraus agierend regelmäßig nicht möglich sein, wenn sie nicht gerade zu einer besonders gut wirtschaftlich oder akademisch vernetzten Gruppe gehören, die bereits Kontakte in den Zielstaat pflegt. Für M besteht daher keine Möglichkeit, bereits ein Arbeitsplatzangebot nachzuweisen.

Hinzu kommt das grundsätzliche Erfordernis der Erteilung einer Berufsausübungserlaubnis nach $\S 18 \mathrm{~V}$ Alt. 2 AufenthG. Unabhängig davon, ob diese mit oder ohne Beteiligung der Bundesagentur für Arbeit ${ }^{25}$ erteilt werden kann liegt hierin ein weiterer möglicher Versagungsgrund, wenn die entscheidende Behörde dies unter Berücksichtigung von $\S 18$ I AufenthG, also der allgemeinen wirtschaftlichen Lage am Arbeitsmarkt, für angemessen hält. Es entfällt lediglich die Zustimmungsbedürftigkeit, nicht die generelle Erlaubnispflicht nach § 4 III 1 AufenthG, sodass im Zweifel die Ausländerbehörde selbst im Wege einer Ermessensentscheidung nach Orientierung an $\S 18$ I AufenthG zu entscheiden hat. ${ }^{26}$

Letzterer Einwand kann entkräftet werden für Hochqualifiziertenmigration, die sich nach $\S 19$ a AufenthG richtet und bei der an die Stelle der Ermessensausübung der Behörde eine gebundene Entscheidung tritt, was grundsätzlich eine Privilegierung der in $\S 19$ a AufenthG geregelten „BlueCard"-Berechtigten darstellt. Jedoch verlangt $\S 19$ a AufenthG neben der allgemeinen Voraussetzung des konkreten Arbeitsplatzangebots ein fixes Mindestgehalt, welches gemäß $§ 2$ I Nr. 2 Beschäftigungsverordnung (BeschV) bei $49.600 € / J a h r$ liegt. ${ }^{27}$ Hierin liegt ein weiteres Erschwernis für Personen, welche - wie $M$ - aufgrund besonders prekärer Lebensumstände migrieren. Zielgruppe der sog. Hochqualifiziertenmigration sind Staaten wie Indien, China, Russland, Ukraine oder die USA, ${ }^{28}$ aus denen regelmäBig die Personen migrieren werden, welche in ihrem Herkunftsstaat bereits

23 Stiegeler, in: Hofmann, Ausländerrecht, § 18 Rn. 22.

24 Breidenbach, in: Kluth/Heusch, Ausländerrecht, § 18 Rn. 47.

25 Vgl. im Einzelnen $\S \S 2 \mathrm{ff}$. BeschV.

26 Breidenbach, aaO; Maor, ZAR 2005, 333 (338).

27 In Mangelberufen 38.688€, vgl. Breidenbach, in: Kluth/Heusch, Ausländerrecht, $\S 19$ a Rn. 13.

28 Migrationsbericht der Bundesregierung, S. 68. 
zur Bildungselite gehören, was schon aus dem Erfordernis der Hochschulbildung des $\S 19$ a I Nr. 1 lit. a) AufenthG folgt.

Völlig aus dem Anwendungsbereich heraus fallen hingegen die Personen, welche über das Mittelmeer migrieren, also vornehmlich Personen aus afrikanischen Herkunftsstaaten. Ebendiese werden, wenn sie nicht bereits daran scheitern, ein Arbeitsplatzangebot vor Ankunft in Europa zu finden, regelmäßig spätestens an der Erreichung der Gehaltsschwelle scheitern. Als nicht hochqualifizierte Person bleibt $M$ auch der Weg über $\S 19 \mathrm{a}$ AufenthG versperrt.

\subsubsection{Aufenthalt zur Arbeitsplatzsuche}

Grundsätzlich kann dem dargestellten Problem des generellen Erfordernisses eines bereits bestehenden Arbeitsplatzangebotes nach $\S 18 \mathrm{~V}$ AufenthG entgegnet werden, dass gerade deshalb $\S 18 \mathrm{c}$ AufenthG geschaffen wurde, welcher die Migration zur Arbeitsplatzsuche für eine Dauer von sechs Monaten ermöglicht - ohne, dass bereits ein Arbeitsplatzangebot in Aussicht stehen muss. Jedoch findet diese Öffnung gemäß $\S 18 \mathrm{c}$ I AufenthG nur Anwendung auf Personen mit deutschem oder diesem vergleichbaren Hochschulabschluss, d.h. hier werden vergleichbar zu § 19a AufenthG lediglich Hochqualifizierte erfasst.

Gerade Personen, welche zurzeit die gefährlichen Migrationsrouten über das Mittelmeer nach Europa nutzen, fallen somit regelmäßig aus dem Anwendungsbereich der $\S \S 18 \mathrm{ff}$. AufenthG Dies spiegelt sich auch in den statistischen Daten des $B A M F$, dass für den gesamten afrikanischen Kontinent lediglich 1-250 Fälle von Arbeitsmigration pro Herkunftsstaat im Jahr 2015 verzeichnet. ${ }^{29}$ Im Vergleich zur gesamten Migration über das Mittelmeer in Höhe von geschätzt über 160.000 Personen allein $2015^{30}$ ist die Arbeitsmigration also verschwindend gering.

\subsection{Irreguläre Migration}

Komplementär zur Arbeitsmigration enthält das Aufenthaltsgesetz auch Regelungen zu irregulärer Migration. Darunter fallen insbesondere geflüch-

29 Vgl. BAMF, Bundesamt in Zahlen, S. 83; wenngleich exakte Daten hier fehlen, liegen alle afrikanischen Staaten jeweils in der untersten Kategorie von 1-250 Personen pro Jahr.

30 Vgl. Frontex, Risk Analysis 2016, S. 16. 
tete Menschen. Für sie wird eine Aufenthaltserlaubnis nach $\S 25$ II AufenthG erteilt, soweit die Flüchtlingseigenschaft nach Maßgabe der Genfer Flüchtlingskonvention oder alternativ subsidiärer Schutz zuerkannt wurde (s.o.). Aufgrund der strengen Selektivität bei der Berücksichtigung von Migrationsgründen, ${ }^{31}$ welche ökonomische und ökologische Motive nicht berücksichtigt, bietet $\S 25$ II AufenthG für diejenigen Personen, welche aus anderen Gründen migrieren keine Perspektive. Als weder individuell verfolgte, noch unter subsidiären Schutz fallende Person hat $M$ daher keinen Anspruch auf Erteilung eines Aufenthaltstitels nach $\S 25$ AufenthG.

Das System der sogenannten humanitären Aufenthaltstitel ist insoweit sehr unflexibel. Unberücksichtigt bleibt, wie stark Migrationsgründe im Einzelnen waren und ob für die betroffenen Personen überhaupt die Option bestand, in ihrer Heimat zu bleiben. Vielmehr wird in der Debatte häufig unterstellt, aus ökonomischen oder ökologischen Gründen erfolgende Migration sei per se freiwillig. ${ }^{32}$ Unabhängig davon, ob man dieser Position zustimmt oder nicht, ist festzustellen, dass im derzeitigen System kein Raum für eine Prüfung der Äquivalenz der Migrationsmotive und einer daran angepassten Einzelfallprüfung ist.

Das negiert die Möglichkeit, dass sich seit dem Inkrafttreten der Genfer Flüchtlingskonvention 1951 die äußeren Umstände und Bedingungen von Migration geändert haben und eine ausschließliche Anwendung dieser Normen die Fluchtursachen des 21. Jahrhunderts nicht mehr vollständig abbildet.

Hinsichtlich irregulärer Migration sind die beiden Kategorien Flüchtlingsstatus und subsidiärer Schutz die einzigen Möglichkeiten zur Erlangung eines Aufenthaltstitels. Somit besteht für $M$ endgültig keine Chance auf den Erhalt eines Aufenthaltstitels. Nach $\S 50$ I AufenthG wäre er daher ausreisepflichtig.

Die hier am Beispiel von $M$ erfolgte exemplarische und teils vereinfachende Darstellung eines Einzelfalls steht damit stellvertretend für eine Vielzahl von Personen, die weder im Rechtsregime des Asylrechts noch des Einwanderungsrechts Berücksichtigung finden und bildet damit die Grund-

31 Vgl. § 3 I AsylG für Flüchtlingsschutz bzw. § 4 I AsylG für subsidiären Schutz.

32 Angenendt, Irreguläre Migration als Konzept, S. 11. 
lage für die folgenden Erwägungen zum Reformbedarf des Migrationsrechts.

\section{KONSEQUENZEN DER KATEGORISIERUNG}

Versucht man die überblicksartig dargestellten Wege zur Erlangung eines legalen Aufenthalts in ein größeres Bild zu fassen, so ergibt sich nicht nur die Unterscheidung zwischen regulärer und irregulärer Migration, sondern zugleich ein Auseinanderfallen von zielstaatsorientierter und individualistischer Betrachtung. Während es sich im Asylrecht verbietet, nach ökonomischem Nutzen für das Zielland der Migrierenden zu unterscheiden, ist dieser bei der regulären Migration zentrales - und nicht selten alleiniges - Kriterium. $^{33}$

Selbstverständlich lassen sich auch Aussagen über die Kosten oder den wirtschaftlichen Nutzen von Geflüchteten für eine Volkswirtschaft treffen. ${ }^{34}$ Diese sind jedoch nach geltendem Recht der Genfer Flüchtlingskonvention in keinem Fall entscheidungserheblich für die Gewährung von internationalem Schutz. Entscheidend ist allein die individuelle Betroffenheit von Verfolgung (s.o.).

Andererseits berücksichtigt das derzeitige System der regulären Migration zum Zweck der Arbeit oder der Ausbildung in keinster Weise humanitäre Gründe. Es lässt sich zwar nicht behaupten, das Individuum werde gänzlich ausgeblendet. Die Auswahl von Personen, denen ein Aufenthaltstitel gewährt wird, erfolgt jedoch stark zielstaatsorientiert insofern, dass in der Regel ein konkretes Arbeitsplatzangebot gefordert wird. Ausnahmen von dieser Regel, wie etwa in $\S 18 \mathrm{c}$ AufenthG, stellen zwar auf persönliche Eigenschaften des Migranten ab, fokussieren sich jedoch weiterhin auf dessen potenziellen wirtschaftlichen Nutzen für den Zielstaat. Unberücksichtigt bleiben stets Migrationsgründe, die allein in der Situation im Herkunftsstaat oder des persönlichen Schicksals der Migranten begründet sind und sich nicht als quantifizierbares Humankapital abbilden lassen.

33 Siehe hierzu den Beitrag von Hochleitner in diesem Band.

$34 \mathrm{Zu}$ dieser Unterscheidung aus einer stärker ökonomischen Perspektive, vergleiche auch hier den Beitrag von Hochleitner in diesem Band. 
Es stehen sich also zwei komplementäre Systeme im Aufenthaltsgesetz gegenüber, die scheinbar nicht miteinander vereinbar sind. Rechtlich schließt die Durchführung eines Asylverfahrens gem. $§ 10$ I den Übergang in einen anderen Aufenthaltstitel aus. Es ist also kein gleichzeitiger Antrag verschiedener Titel möglich, was verfahrensökonomisch auch richtig ist. Auch ein Übergang nach abgeschlossenem negativ beschiedenem Asylverfahren in die Arbeitsmigration ist jedoch nach $\S 10$ III AufenthG nicht möglich. Eine solche sog. „Spurwechsel“-Option wird dahingegen vielfach als mögliche Reform diskutiert, politisch aber mehrheitlich abgelehnt. ${ }^{35}$

Im Gegensatz zur rechtlichen Trennung findet in tatsächlicher Hinsicht hingegen eine deutlich stärkere Vermischung von Flucht und Migration statt. Bereits ab dem Aufbruch aus ihrem Heimatstaat nutzen Flüchtlinge und andere irreguläre Migranten vielfach dieselben Netzwerke und Migrationsrouten. ${ }^{36}$ Es wird von dem Phänomen der ,,mixed migration“ gesprochen. ${ }^{37}$ Zudem ist es nicht unwahrscheinlich, dass Asylbewerber während des andauernden Verfahrens eine Arbeitsstelle in Deutschland finden, jedoch wegen $\S 10$ III AufenthG nicht den Aufenthaltszweck wechseln können. Eine klare Trennung in zwei abgrenzbare Gruppen, wie sie das Aufenthaltsgesetz vornimmt, ist an realen Lebenssachverhalten kaum nachvollziehbar.

Betrachtet man zudem die Migrationsgründe von nach Europa kommenden Personen, so ergibt sich ein Motivbündel aus wirtschaftlichem Anreiz, sich eine bessere Zukunft in Europa erarbeiten zu können und Faktoren, die in der Situation des Heimatstaates bedingt sind, wie etwa schlechter Versorgungslage oder (politischer) Verfolgung. ${ }^{38}$ Eine abschließende Unterscheidung in „Push- und Pull-Faktoren“ erscheint bereits aufgrund dieser Gemengelage an unterschiedlichen Migrationsgründen nicht zielführend. Vielmehr werden diese sich stets gegenseitig überlagern. ${ }^{39}$ Dieser Umstand

35 Parusel, ZAR 2014, 115ff.; Berlit, ZAR 2018, 229 (236).

36 Angenendt, Irreguläre Migration als internationales Problem, S. 12.

37 Vgl. UNHCR, Refugee Protection and Mixed Migration, S. 1f.

38 Empirisch dazu Crawley/Skleparis, Journal of Ethnic and Migration Studies, S. 48 (55).

39 Crawley/Skleparis, aaO, S. 50. 
trägt zu einem weiteren Verschwimmen der Grenzen zwischen den einst ${ }^{40}$ klar abgrenzbaren Kategorien regulärer und irregulärer Migration bei.

Diese Komplexität wird jedoch im geltenden Recht nicht abgebildet. Stattdessen wird für den Großteil der irregulär migrierenden Personen ein Asylverfahren durchgeführt, welches aus Gründen der oben dargestellten Selektivität der anerkannten Fluchtursachen regelmäßig negativ endet. Damit kommt es zum Folgeproblem der Illegalisierung des Aufenthalts und ggf. notwendiger Rückführung oder jedenfalls der Ausreise, um überhaupt einen neuen Antrag nach $\S \S 17,18 \mathrm{ff}$. AufenthG stellen zu können. Ein fließender Übergang, etwa im Rahmen des sog. Spurwechsels wird dabei durch $\S 10$ III 1 AufenthG verhindert.

Konsequenz aus dem Mangel an gesetzlichen Regelungen zu gemischter Migration ist die generelle Einordnung als irreguläre Migration. Damit wird dem Dogma gefolgt, dass irreguläre Migration grundsätzlich in jedem Rechtssystem und insbesondere in Staaten mit einer hohen Regulierungsdichte eine unwillkommene Restgruppe darstellt, welche es $\mathrm{zu}$ reduzieren gilt. $^{41}$

Fraglich und umstritten ist indes, auf welchem Weg eine Reduktion der irregulären Migration stattfinden kann. Häufig wird dabei eine Dichotomie zwischen der viel beschworenen Bekämpfung der sogenannten „Fluchtursachen" und der Abschottung bzw. Erschwerung des Grenzübertritts vorausgesetzt. Ein effektives Mittel zur Bekämpfung der hohen Zahlen irregulärer Migration wäre hingegen auch eine voranschreitende Legalisierung von Migrationsbewegungen nach zuvor festgelegten einheitlichen Kriterien.

40 Auf theoretischer Ebene schließen sich die Begriffe aus. Schwierigkeiten ergeben sich erst in der Anwendung auf reale Sachverhalte, die eine klare Zuordnung von Personen in eine der Kategorien oft unmöglich machen.

41 Dazu Angenendt, Irreguläre Migration als internationales Problem, S. 7; zu gesetzgeberischen Trends der Unterbindung Irregulärer Migration, vgl. de Haas, Growing Restrictiveness or Changing Selection?, S. 25. 


\section{LEGALE ZUGANGSWEGE ALS MITTEL ZUR REGULARISIERUNG}

Möglicherweise kann also mit geschickter Rechtssetzung selbst ein Beitrag zur Verringerung irregulärer Migration geleistet werden. Auch die Forderung nach mehr legalen Zugangswegen nach Europa ist schließlich nichts anderes als eine Forderung nach einer Verschiebung von irregulärer Migration zu regulärer Migration. Man kann insoweit auch von einer Verrechtlichung oder Regularisierung der irregulären Migration sprechen.

Es ist insofern zu fragen, ob ein möglicher Weg der Regularisierung die Öffnung und Flexibilisierung der derzeit bestehenden Tatbestände regulärer Migration wäre.

\subsection{These: Derzeitige Rechtslage bereits ausreichend}

Teils wird vertreten, das Aufenthaltsgesetz sei in der aktuellen Fassung mit den Möglichkeiten, welche es zur Migration von Fachkräften biete ausreichend und sollte daher auch abschließend sein. ${ }^{42}$

Derzeit wird wie gezeigt jedoch lediglich Hochqualifiziertenmigration von den $\S \S 18 \mathrm{ff}$. AufenthG erfasst. Stellt man sich auf den Standpunkt, dass sich die deutsche Migrationskontrolle darauf beschränken solle, spricht tatsächlich vieles dafür, das Aufenthaltsgesetz als hinreichende Regelung zu betrachten. $^{43}$

Zum einen handelt es sich um ein in sich stimmiges und abgeschlossenes Regelungssystem. ${ }^{44}$ Auch die europarechtlichen Vorgaben, insbesondere der Blue-Card-Richtlinie wurden entsprechend umgesetzt. ${ }^{45}$ Aufgrund des wie gezeigt geringen und regional äußerst selektiven Anwendungsbereichs und der weiterhin hohen irregulären Migration, insbesondere über

42 Klaus, ZAR 2017, 60 (65); allein semantische Änderungen hält etwa Thym, ZAR 2018, 193 (199) für sinnvoll.

43 Vgl. so etwa Döring, NVwZ 2016, 1033 (1038).

44 SVR, Jahresgutachten 2014, S. 15.

45 Herzog-Schmidt, Die Blue-Card Richtlinie und ihre Umsetzung in Deutschland, S. $265 \mathrm{ff}$. 
das Mittelmeer, ist jedoch $\mathrm{zu}$ fragen, ob nicht darüber hinaus vertretbare Möglichkeiten zur Öffnung des Migrationsrechts bestehen.

\subsection{Ausweitung des Anwendungsbereichs der Blue-Card-Richtlinie}

Hier setzt auch der europäische Rat mit dem Entwurf einer Novellierung der Blue-Card-Richtlinie an. ${ }^{46}$ Die wesentlichen Änderungen im europäischen Gesetzgebungsvorschlag beziehen sich dabei auf eine Herabsetzung der Mindestgehaltsschwellen und Vereinfachungen im Verwaltungsverfahren. $^{47}$

Unverändert bliebe jedoch die Beschränkung der Blue-Card auf Personen, welche bereits bei der Bewerbung um einen Aufenthaltstitel die konkrete Zusage für einen Arbeitsplatz in der EU erhalten haben. ${ }^{48}$ Trotz gleichzeitiger Erweiterung der Ausschlusstatbestände ${ }^{49}$ ist dadurch zu erwarten, dass die Zahl erteilter „Blue-Cards“ zunehmen wird. Jedoch handelt es sich weiterhin um einen Ansatz, der ausschließlich Personen erfasst, welche bereits Netzwerke in Europa haben oder jedenfalls die Voraussetzungen mitbringen, Kontakte zu zukünftigen Arbeitgebern aus ihrem Heimatland heraus aufzubauen. ${ }^{50}$ Daher ist die Regelung weiterhin selektiv insoweit, dass sie vornehmlich bereits gesellschaftlich privilegierte Gruppen anspricht. Eine maßgebliche Erweiterung des räumlichen Anwendungsbereichs über die USA und BRICS-Staaten hinaus ist somit unter Berücksichtigung der statistischen Daten ${ }^{51}$ nicht zu erwarten.

Aus diesen Gründen ist vom europäischen Gesetzgebungsvorschlag zwar zu erwarten, dass er quantitativ zu einer Erhöhung der Einwanderung von auf dem deutschen und europäischen Arbeitsmarkt nachgefragten Personen führen wird. Qualitativ hingegen ist keine Abkehr vom bisherigen streng arbeitsmarktorientierten Ansatz der Migrationskontrolle erkennbar.

46 Europäische Kommission, $\operatorname{COM(2016)~} 378$.

47 Klaus, ZAR 2017, 60 (67f.)

48 Klaus, ZAR 2017, 60 (65).

49 Klaus, ZAR 2017, 60 (66).

50 Vgl. dazu oben Fn. 31, 32.

51 Vgl. BAMF, Bundesamt in Zahlen, S. 85. 
Daher wäre auch bei Umsetzung des Gesetzesvorschlags eine Senkung der irregulären Migration (über das Mittelmeer) nicht zu erwarten.

Wenngleich lediglich Prognosen über wirksame Alternativen möglich sind, kann jedenfalls gesagt werden, dass zur Verringerung der irregulären Migration ein inklusiverer Ansatz notwendig ist, der rechtliche und verwaltungstechnische Aspekte vereint. Diskutiert wird hierzu etwa die vereinfachte Registrierung und Aufnahme migrationswilliger Personen in eine Datenbank, aus der sie sodann direkt von Unternehmen in potenziellen Zielstaaten angeworben werden könnten. ${ }^{52}$ Zudem wären Vereinfachungen auf der unteren Verwaltungsebene denkbar. So haben viele Personen keinen oder nur erschwerten Zugang zum Visaverfahren aufgrund geografischer Faktoren oder mangelnder personeller Ausstattung der zuständigen Botschaften. $^{53}$

\subsection{Nationales Punktesystems}

Um jedoch realistisch und unmittelbar Einfluss auf das Phänomen der gemischten Migration zu haben bedarf es eines flexibleren und anpassungsfähigeren Systems der Migrationskontrolle.

Systemisch bietet ein bereits in Gesetzgebungsvorschlägen deutscher Parteien genanntes ${ }^{54}$ Punktesystem zur Migrationskontrolle den benötigten Freiraum zur Implementierung neuer Kriterien zur bewussten Aufnahme bestimmter Personengruppen. Freilich ist damit noch nicht die Frage beantwortet, welche die entscheidenden Kriterien eines solchen Systems sein könnten. Die Einführung eines Punktesystems hat somit keinen Wert für sich und kann stets nur die Grundlage für Veränderungen und eine etwaige Modernisierung des Migrationsrechts darstellen. Insbesondere sind etwaige Vorschläge nicht primär danach zu bewerten, wie sehr sie das Migrationsrecht vereinfachen oder transparenter machen können, sondern zunächst danach, ob sie materiell die sich politisch gesetzten Ziele erreichen können.

52 Angenendt/Kipp/Meier, Ansätze zur Bewältigung gemischter Wanderungen, S. 39.

53 Angenendt/Kipp/Meier, a.a.O, S. 38f.

54 SPD-Bundestagsfraktion, Entwurf eines Einwanderungsgesetzes (EinwG-E) v. 7.11.2016; B90/Die Grünen, EinwG-E, BT-Drs. 18/11854; vgl. dazu: Hörich, ZRP 2017, 154; Kolb/Lehner, ZAR 2017, $270 \mathrm{ff}$. 
Schließlich kann die Vereinfachung des Rechts ein politisches Ziel sein. Sie sollte dabei aber nicht unhinterfragt und nicht auf Kosten inhaltlicher Ziele geschehen. $^{55}$

\subsubsection{Mögliche Kriterien eines Punktesystems}

Bedeutsam bleibt die Frage, nach welchen Kriterien die Beurteilung der Möglichkeit der Erteilung eines Aufenthaltstitels erfolgen soll. Letztlich stellt dies eine Frage dar, die vom parlamentarischen Gesetzgeber oder einer demokratisch legitimierten Kommission ${ }^{56} \mathrm{zu}$ beantworten sein würde. Im Folgenden sollen lediglich verschiedene mögliche Ansätze dargestellt und analysiert werden, um Vorteile und Maximen der Punkteregelung herauszuarbeiten.

Ein allen bestehenden Vorschlägen eines Punktesystems gemeinsamer Ansatz ist die Erweiterung der Arbeitsmarktanbindung um humankapitalorientierte Zuwanderung. Dies zeigt sich dadurch, dass auch ohne bestehendes Arbeitsplatzangebot bei Vorliegen von formalen Kriterien wie einem Hochschulabschluss oder einer qualifizierten Berufsausbildung die nötige Punktzahl erreicht werden kann. ${ }^{57}$ Es wird also davon ausgegangen, dass Personen mit einer nachgewiesenen formellen Qualifikation auch einen Arbeitsplatz finden werden, ihnen also Zugang zum Arbeitsmarkt gewährt werden sollte. Insofern handelt es sich um eine arbeitsmarktpolitische Prognose. Wenngleich keine vollständige Abkehr vom arbeitsmarktorientierten Modell der $\S 18 \mathrm{ff}$. AufenthG vorliegt, sofern weiterhin Punkte für ein Arbeitsplatzangebot vergeben werden, ${ }^{58}$ so ist es doch möglich durch Qualifikation (ggf. ergänzt durch weitere Faktoren) diesen Mangel auszugleichen.

Ergänzt wird die Gewichtung der Qualifikation durch verschiedene Faktoren, welche sich auf das Potenzial der Einwandernden beziehen, sich im Arbeitsmarkt und Gesellschaft zu integrieren. Insbesondere werden als

55 Offer, ZAR 2017, 29 (30).

56 Eine Einwanderungskommission fordert § 19 II EinwG-E (Grüne).

57 Vgl. die Beispielrechnungen bei Hunger/Krannich, WISO 05/2017, S. 27, S. 34.

58 Anlage 1 und 2 zu EinwG-E (SPD); in der Höhe variierend zu anderen Vorschlägen in der Debatte, vgl. dazu die Gegenüberstellung bei Hunger/Krannich, a.a.O. 
Indikatoren hierfür niedriges Alter und hohe Deutschkenntnisse genannt. ${ }^{59}$ Teils seien auch in geringem Maß bestehende Bindungen zu Deutschland als positive Integrationsaspekte zu berücksichtigen. ${ }^{60}$ Die hierdurch charakterisierten Gruppen sind insbesondere geeignet, sich in Deutschland ausund weiterzubilden. Bildungsmigration zu erleichtern ist ein (jedenfalls sekundäres) Ziel der Gesetzgebungsvorschläge. ${ }^{61}$

\subsubsection{Kritik}

Die grundsätzlich begrüßenswerten Ansätze zur Flexibilisierung des Einwanderungsrechts stoßen jedoch auf vielfältige Kritik.

Deutschland war verpflichtet die sogenannte Blue-Card-Richtlinie ${ }^{62}$ umzusetzen, was im Aufenthaltsgesetz, insbesondere durch die Schaffung des Aufenthaltstitels des $\S 19$ a geschehen ist. Eine Änderung des Aufenthaltsgesetzes als Umsetzung der europäischen Richtlinie steht nur insoweit zur Disposition des nationalen Gesetzgebers, als dadurch die Umsetzung der Richtlinie nicht gefährdet wird.

Bei der rechtlichen Beurteilung der Implementierung eines Punktesystems sind insoweit zwei Szenarien zu unterscheiden: die Ergänzung des jetzigen Einwanderungsrechts um ein Punktesystem als zusätzlichen Zuwanderungskanal und die Neu-Konzeptionierung des Einwanderungsrechts nach dem Modell eines Punktesystems unter Aufhebung der bisherigen Vorschriften. $^{63}$

Ein zusätzliches Punktesystem würde die Zuwanderungskontrolle lediglich weiter liberalisieren, indem eine neue Zuwanderungsoption geschaffen würde. Der Zugang über die Blue-Card würde dadurch weder verhindert noch erschwert werden. Ein Exklusivitätserfordernis enthält die Richtlinie 2009/50 gerade nicht. Es wird zwar diskutiert, die Ausgestaltung der Zuwanderungskontrolle auf Grundlage des Art. 79 AEUV noch weitgehender europarechtlich zu fassen. ${ }^{64}$ Etwaige Vorschläge sind jedoch nicht gelten-

59 Hunger/Krannich, WISO 05/2017, S. 27f.

60 So etwa Anlage 1 und 2 zu EinwG-E (SPD).

61 EinwG-E (SPD), S.2; EinwG-E (Grüne), S. 17.

62 Richtlinie 2009/50 (EG).

63 Diese Kategorisierung vollziehen auch Hunger/Krannich, WISO 05/2017, S.24, 30 in Bezug auf aktuelle Gesetzgebungsvorschläge.

64 Vorschlag für eine Neufassung der Richtlinie $\operatorname{COM}(2016)$ 378, S. 2 f. 
des Recht und hindern eine zusätzliche Schaffung eines Punktesystems daher nicht.

Bemerkenswert ist indes, dass von Verfechtern eines Punktesystems gerade auf eine Vereinfachung und Vereinheitlichung der Zuwanderungskontrolle in einem übersichtlicheren, transparenteren System gepocht wird. ${ }^{65}$ Dazu steht ein ergänzendes Punktesystem in klarem Widerspruch, da es statt einer Vereinfachung zu einer weiteren Komplexitätssteigerung im Migrationsrecht führen würde. ${ }^{66}$ Konsequent wäre es daher wohl, eine umfassende Novellierung des Aufenthaltsgesetzes anzustreben.

Für diesen Fall könnte jedoch die Vereinbarkeit mit dem Europarecht in Frage stehen.

Grundsätzlich ist eine Neuregelung des $§ 19 a$ AufenthG der Regelungskompetenz des nationalen Gesetzgebers entzogen. ${ }^{67}$ Möglich wäre es allerdings die Regelung des $\S 19 \mathrm{a}$ AufenthG in einem etwaigen Einwanderungsgesetz zu übernehmen und dort zu integrieren. Es erscheint jedoch nur schwer denkbar, tatsächlich den tendenziell humankapitalorientierten Ansatz eines Punktesystems mit dem starren Kriterium der Arbeitsplatzzusage in $\S 19$ a AufenthG zu kombinieren.

Nimmt man etwa an, es würden diejenigen Personen mit einem ausreichend hohen Punktewert in einen Bewerberpool aufgenommen, aus dem jährlich ein per Rechtsverordnung durch die Bundesregierung festzusetzendes und damit flexibles Kontingent aufzunehmen wäre, ${ }^{68}$ so stünde diese Regelung potenziell in Konflikt mit $\S 19$ a AufenthG. Dieser sieht bei Vorliegen der Tatbestandsvoraussetzungen, also sofern neben den allgemeinen Voraussetzungen $^{69}$ insbesondere die hohe Qualifikation und eine Arbeitsplatzzusage bei entsprechendem Mindestgehalt vorliegen gerade einen Anspruch auf Erteilung einer Aufenthaltserlaubnis vor und ist daher mit einer flexiblen, auf festen Obergrenzen basierenden Kontingentlösung nur schwer vereinbar. Es spricht daher viel dafür, eine Koexistenz von arbeitsmarktorientierten Regelungen nach Modell der Blue-Card in klarer Tren-

65 EinwG-E (SPD), S. 12.

66 Kolb/Lehner, ZRP 2017, 34 (36).

67 Kolb/Lehner, ZAR 2017, 270 (272); dies., ZRP 2017, 34 (36).

68 So der Vorschlag bei: EinwG-E (Grüne), § 19 III; Hunger/Krannich, WISO 05/2017, S. 34.

$69 \mathrm{Vgl}$. dazu $\S 5$ Aufenthaltsgesetz, welcher allgemein für alle Aufenthaltstitel gilt. 
nung zu humankapitalorientierten Ansätzen im Punktesystem anzustreben. $^{70}$

Eine Regelung des Punktesystems in Koexistenz zu den europarechtlich vorgegebenen Aufnahmetatbeständen, erscheint also möglich. Dennoch bleibt zu klären, ob das Ziel einer verstärkten humankapitalorientierten Zuwanderung nicht bereits im jetzigen Recht möglich ist oder auf einfacherem Wege ausgehend von einer Novellierung einzelner Normen erreicht werden könnte.

So wird argumentiert, es bestünde bereits abseits der streng arbeitsmarktorientierten Zuwanderung mit $\S \S 16$ IV, 17 III, 18c AufenthG die Möglichkeit zur Migration lediglich aufgrund einer positiven Prognose wie sie auch das Punktesystem anhand bestimmter Kriterien, anknüpfend an Alter, Sprachkenntnisse, Bildungsstand oder sonstiger Qualifikationen vornehmen will. ${ }^{71}$ Prognostisch sind diese Ansätze insoweit, da sie mithilfe von objektivierten Kriterien versuchen zu ermitteln, inwieweit sowohl migrationswillige Personen wie auch der Zielstaat in Zukunft von der Migrationsbewegung profitieren werden. Daher lässt sich dieser Ansatz auch als potenzialorientiert, als Unterkategorie humankapitalorientierter Ansätze, bezeichnen.

Der Wirksamkeit der $\S \S 16$ IV, 17 II AufenthG ist jedoch entgegenzuhalten, dass lediglich ein Aufenthaltstitel im Anschluss an einen bereits bestehenden Aufenthalt ermöglicht wird. Die initiale Migration im Sinne der Einwanderung ist hiermit jedoch gerade nicht erfasst.

$\S 18 \mathrm{c}$ AufenthG hingegen bietet tatsächlich die Möglichkeit der Potenzialzuwanderung. Erforderlich ist für die Erteilung grundsätzlich nur die formelle hohe Qualifikation und gerade nicht ein bestehender Arbeitsplatz. Die Regelung vermag die Gruppe der Personen mit Hochschulabschluss, die auch vom Punktesystem privilegiert werden, zu erfassen. Jedoch sind entgegen des Punktesystems bereits durch Berufsausbildung oder Berufserfahrung gewonnene Qualifikationen von $\S 18 \mathrm{c}$ AufenthG nicht mehr erfasst. Zudem ist die maximale Befristungsdauer von sechs Monaten sehr kurz und auch die Erwerbsmöglichkeit ist währenddessen nicht gegeben. Im Ergebnis wird $\S 18 \mathrm{c}$ AufenthG tatsächlich aufgrund der hohen Hürden

70 So unter Analyse einzelner möglicher Fallgruppen auch Kolb/Lehner, ZRP 2017, 34 (37).

71 Dörig, NVwZ 2016, 1033 (1035). 
nur relativ wenig in Anspruch genommen. ${ }^{72}$ Jedenfalls in der jetzigen Fassung kann in $\S 18 \mathrm{c}$ AufenthG daher keine dem Ansatz des Punktesystems gleichwertige Regelung gesehen werden.

\subsubsection{Flexibilisierung und Effektivitätssteigerungen ausgehend von § 18c AufenthG}

Vorschnell ist jedoch der daraus gezogene Schluss, es sei eine vollkommene Neufassung und Aufhebung des $\S 18 \mathrm{c}$ AufenthG erforderlich. ${ }^{73}$

Indes sollte vorrangig erwogen werden, inwiefern nicht das Ziel einer flexibleren und effektiveren Zuwanderungskontrolle mittels oder durch Änderung bestehender Regelungen erreicht werden kann. § 18c AufenthG stellt eine überschießende Umsetzung der Blue-Card Richtlinie (2009) dar. ${ }^{74}$ Der geschaffene Tatbestand eines Aufenthalts zur Arbeitsplatzsuche kann durch den nationalen Gesetzgeber daher auch in einem einfachen Gesetzgebungsverfahren geändert und gegebenenfalls geöffnet werden.

Die derzeitige kurze und zwingende Befristung in $\S 18 \mathrm{c} \mathrm{I} 1$ AufenthG auf maximal sechs Monate könnte erhöht werden etwa auf ein Jahr oder 18 Monate, wobei die Entscheidung dem Ermessen der Ausländerbehörde unterliegen kann, sodass im Einzelfall Gründe, wie eine besondere Härte oder besonders aufwändige Arbeitsplatzsuche berücksichtigt werden können. Die grundsätzliche Existenz einer Befristung ist jedoch essentiell, um eine „Endlossuche“ nach einem Arbeitsplatz und damit eine Verfestigung des stets nur als Übergangsregelung gedachten $\S 18 \mathrm{c}$ zu verhindern. ${ }^{75}$

Durch Änderung des $§ 18 \mathrm{c}$ I 2 AufenthG könnte überdies die Beschäftigung erlaubt werden, um dem Betroffenen zu ermöglichen, neben der Arbeitsplatzsuche (jedenfalls teilweise) seinen Lebensunterhalt zu finanzieren. Denkbar wäre infolgedessen auch eine Berücksichtigung der potenziellen Erwerbstätigkeit im Rahmen der Prüfung nach § 18c I 1 AufenthG, ob der Lebensunterhalt gesichert ist. ${ }^{76}$ Ein Verzicht auf das Erfordernis des Nachweises entsprechender finanzieller Rücklagen erscheint allerdings allein darauf gestützt nicht adäquat, da schließlich aufgrund einer Prognose nicht si-

72 Im Jahr 2015 von etwa 1500 Personen, vgl. BT-DrS 18/11854, S. 18.

73 So aber EinwG-E (Grüne), S. 18.

74 Breidenbach, in: Kluth/Heusch, Ausländerrecht, § 18c vor Rn. 1.

75 Kolb/Lehner, ZAR 2017, 270 (273).

76 In diese Richtung bereits weist bereits das SVR Jahresgutachten 2014, S. 76. 
chergestellt ist, dass tatsächlich eine geringfügige Beschäftigung aufgenommen wird. Eine vollständige Ausnahme ließe sich lediglich rechtfertigen, soweit bereits bei Bewerbung eine Zusage zur finanziellen Unterstützung des Mobilitätsvorhabens vorliegt. ${ }^{77}$

Denkbar wäre eine Öffnung des $\S 18 \mathrm{c}$ AufenthG auch für Personen mit einer abgeschlossenen qualifizierten Berufsausbildung oder auch für Ausbildungssuchende. $^{78}$

Zwar sieht § 17 AufenthG Möglichkeiten zum Aufenthalt zum Zwecke der Berufsausbildung ähnlich wie $\S 16$ AufenthG für ein Studium vor. Jedoch muss auch hier bereits ein Ausbildungsplatz vorliegen, ähnlich wie bei $\S 18$ AufenthG das Arbeitsplatzangebot. Im Fall der Erwerbsmigration wurde auf die daraus resultierenden Schwierigkeiten mit der Einführung des $\S 18 \mathrm{c}$ AufenthG reagiert. Es erscheint daher naheliegend, einen solchen Schritt auch für Ausbildungssuchende zu erwägen. Um die Äquivalenz mit dem Kriterium des Hochschulabschlusses in $\S 18 \mathrm{c}$ I AufenthG hinsichtlich der zu erwartenden positiven Arbeitsmarktintegration zu wahren, könnte der Anwendungsbereich etwa auf Personen mit relevanter Vorbildung beschränkt werden und darüber hinaus wäre es denkbar, Ausbildungssuchende in Branchen der Engpassberufe zu bevorzugen. Es drängt sich jedoch bereits auf, dass die Auswahl gerade auf Verwaltungsebene mangels klar vergleichbarer und bestimmbarer Kriterien problematisch werden könnte.

Es wäre demgegenüber auch möglich, § 18c I AufenthG insoweit zu erweitern, dass statt des Hochschulabschlusses, Personen mit abgeschlossener qualifizierter Berufsausbildung ihre hohe Qualifikation durch eine im Heimatland abgelegte berufsqualifizierende Prüfung und mehrjährige Arbeitserfahrung nachweisen können. Gegebenenfalls kann und sollte auch diese partielle Erweiterung auf Berufsausbildungen in Engpassberufen beschränkt werden.

Letztlich ist kurz auf den Ausschlusstatbestand des § 18c III AufenthG einzugehen. Wenngleich dieser lediglich auf Personen anwendbar ist, welche sich bereits in Deutschland aufhalten und somit keine Auswirkung auf die Migrationsbewegung selbst hat, wäre es doch erwägenswert bei einer Neufassung des $§ 18 \mathrm{c}$ den Absatz III zu streichen. Er läuft dem eigentlichen

77 Denkbar wären Stipendien oder Förderprogramme zur Aus- und Fortbildung.

78 So bereits ein Vorschlag im SVR Jahresgutachten 2014, S. 76; zustimmend Bünte/Knödler, ZRP 2018, 102 (105); Kluth, NVwZ 2018, 1437 (1441). 
Zweck der Norm, der Bindung von Fachkräften an den deutschen Arbeitsmarkt, entgegen, indem er im Fall der plötzlichen Beendigung ohne sofortige Weiterbeschäftigung und damit dem Wegfall des Aufenthalts nach § 18, den Weg zu einem Aufenthaltstitel zur Arbeitsplatzsuche versperrt. Es wird zwar argumentiert, der Wortlaut des $\S 18 \mathrm{c}$ III sei in historischer Auslegung restriktiv zu verstehen. ${ }^{79}$ Konsequent im Sinne einer Attraktivitätssteigerung Deutschlands als Einwanderungsland wäre es jedoch, den Ausschlusstatbestand zu streichen. Dem Gesetzeszweck der Einhaltung etwaiger Höchstarbeitszeiten nach der BeschV ${ }^{80}$ kann schließlich problemlos im Wege der Ermessensentscheidung der zuständigen Behörde entsprochen werden.

\subsection{4 „Westbalkan-Regelung“ als Alternativkonzept}

Beachtenswert ist die im Oktober 2015 vorgenommene Öffnung des $§ 26$ I BeschV für niedrigqualifizierte Arbeitnehmer. Fraglich ist, ob dies eine tragfähige Alternative zur Ausweitung des $\S 18 \mathrm{c}$ AufenthG darstellen kann. Für die Länder des Westbalkans wird auf den Nachweis einer besonderen Qualifikation wie sonst etwa in $\S \S 2 \mathrm{ff}$. BeschV verzichtet. Es handelt sich um eine erhebliche Zuwanderungserleichterung für die entsprechenden Staaten, die jedoch anders als die genannten Vorschläge keinen Bezug auf besondere Qualifikationen oder sonstige Merkmale der Arbeitnehmer nimmt. Lediglich ein konkretes Arbeitsplatzangebot ist weiterhin notwendig, vgl. § 18 V AufenthG.

Die Regelung des $\S 26$ II BeschV ist damit vor allem eine Öffnung für Migration in den Niedriglohnsektor. ${ }^{81}$ Eine länderunabhängige Erweiterung der Norm erscheint daher arbeitsmarktpolitisch riskant, wenngleich zu beachten ist, dass weiterhin die Zustimmung durch die Bundesagentur für Arbeit notwendig ist, was eine Kontrolle und Feinsteuerung ermöglicht.

Wie die regionale Selektivität in Bezug auf den Westbalkan politisch gerechtfertigt werden soll verbleibt zudem fraglich. § 26 II BeschV wirkt damit wie ein Fremdkörper im ansonsten weitgehend systematisch stimmigen System des Migrationsrechts. Statt einer partiellen Aufhebung der Zu-

79 Strunden/Schubert, ZAR 2012, 270 (272).

80 Vgl. zum Gesetzeszweck Breidenbach, in: Kluth/Heusch, Ausländerrecht, § 18c Rn. 5.

81 Kritisch auch Langenfeld, ZRP 2015, 193. 
wanderungskotrolle wäre es weitaus konsistenter, $\S 18 \mathrm{c}$ wie ausgeführt zu öffnen und damit Raum für eine kontrollierte und gesteuerte schrittweise Erhöhung der Zuwanderung - ggf. auch in nicht-akademische Berufe - zu ermöglichen.

\subsubsection{Punktesystem trotz $\S 18 \mathrm{c}$ Aufenth}

Wenngleich viel für die Öffnung des Einwanderungsrechts in Richtung einer stärkeren potenzialorientierten Zuwanderung über $\S 18 \mathrm{c}$ AufenthG spricht, ist zu fragen, ob daneben noch Raum für ein Punktesystem besteht. Von allen Vorschlägen unberücksichtigt bleiben schließlich weiterhin Faktoren wie ökonomische oder ökologische Migrationsgründe.

Durch die Berücksichtigung humanitärer Gründe eine umfassende Antwort auf das Phänomen der gemischten Wanderungen zu bieten, wäre gerade das große Potenzial eines flexiblen Punktesystems. ${ }^{82}$ So wäre es möglich, gemischte Wanderungen adäquat abzubilden, indem eine rechtliche Verstrickung von humanitären und zielstaatsorientierten Kriterien in die Zuwanderungssteuerung einfließen könnte. Etwa könnten bestehende Vorschläge eines Punktesystems um Kategorien wie ökologische oder ökonomische Ursachen ergänzt werden. Diese wären dann im Einzelfall im Wege einer Ermessensentscheidung der erkennenden Behörde zu berücksichtigen. Von vornherein klar ist, dass hierdurch nicht die bestehenden rechtlichen Pflichten zur Gewährleistung von internationalem Schutz ersetzt werden könnten. Vielmehr könnte eine etwaige Regelung eine Lücke im bestehenden Recht schließen, die daraus entsteht, dass die anerkannten Fluchtgründe auf der Genfer Flüchtlingskonvention, einem völkerrechtlichen Übereinkommen aus dem Jahr 1951, basieren, die seitdem in ihrem sachlichen Anwendungsbereich nicht angepasst wurde. Eine Berücksichtigung der heutigen Umstände der Zeit erscheint geboten, sofern man entsprechende Ungleichbehandlungen irregulärer Migranten nicht hinnehmen möchte.

Vor dem Hintergrund, dass jedoch eine originäre Anpassung der Genfer Flüchtlingskonvention in weiter Ferne jedweder Debatte liegt, erscheint die Erweiterung eines Punktesystems um humanitäre Gründe einen vor dem Hintergrund der gemischten Wanderungen und von einem menschenrechts-

82 So im Ansatz auch Angenendt/Kipp/Meier, Gemischte Wanderungen, S. 40. 
orientierten Standpunkt her wünschenswerten, aber in näherer Zukunft nicht zu erwartenden politischen Kurswechsel darzustellen.

\section{FAZIT}

Es ist festzustellen, dass im Wege einer Öffnung des deutschen Migrationsrechts für angebotsorientierte Einwanderung die Attraktivität Deutschlands als Einwanderungsland steigen würde. Dies wird unterstützt durch etwaige Erweiterungen des bedarfsorientierten Ansatzes auf europäischer Ebene in der Blue-Card-Richtlinie. Für eine Ausrichtung als modernes Einwanderungsland mit einer Kombination aus angebotsorientiertem und bedarfsorientiertem Einwanderungsrecht ist die Einführung eines Punktesystems nicht notwendig. Vielmehr kann dies mit einer entsprechenden Öffnung des $\S 18 \mathrm{c}$ AufenthG einfacher und kohärenter erreicht werden.

Anders läge der Fall, würde das Punktesystem auch humanitäre Gründe berücksichtigen. Sodann könnte es adäquat in rechtlicher Hinsicht das Phänomen der gemischten Wanderungen abbilden und böte die Chance, derzeit irregulär stattfindende Migration weitgehend zu regularisieren. Nach den derzeit bekannten Entwürfen für Punktesysteme finden humanitäre Gründe jedoch keine Berücksichtigung, sodass ein Mehrwert zu der Regelung über eine Novellierung des $\S 18 \mathrm{c}$ nicht ersichtlich ist.

$\mathrm{Zu}$ einer Entflechtung der gemischten Wanderungen kann eine Öffnung des Arbeitsmigrationsrechts nur partiell beitragen. Grundsätzlich wird irreguläre Migration durch eine Regularisierung und die Vereinfachung des Zugangs zu Wegen regulärer Migration verringert. Es kann aber nicht mit hinreichender Wahrscheinlichkeit prognostiziert werden, inwiefern die Zahlen irregulärer Migration tatsächlich zurückgehen würden.

Entscheidend für den Erfolg eines attraktiven Arbeitsmigrationsrechts ist neben der Schaffung rechtlicher Grundlagen vor allem die effektive Kommunikation und Bewerbung Deutschlands als Einwanderungsland. Die Verantwortung hierfür liegt insbesondere bei den deutschen Auslandsvertretungen, wie auch bei entsprechenden Mittler- und Durchführungsorganisationen. Neben einer offensiveren Kommunikation lässt auch eine Vereinfachung der Verwaltungsverfahren Effektivitätssteigerungen erwarten. 


\section{LITERATUR}

Angenendt, Steffen, Irreguläre Migration als internationales Prob-lem, SWP-Studie 2007/S 33.

Angenendt, Steffen/Kipp, David/Meier, Amrei, Gemischte Wanderungen, Bertelsmann Stiftung 2017.

Berlit, Uwe, Migration und ihre Folgen - Wie kann das Recht Zuwanderung und Integration in Gesellschaft, Arbeitsmarkt und Sozialordnung steuern? (Teil 1), in: ZAR 2018, S. 229-238.

Bundesamt für Migration für Flüchtlinge (BAMF), Migrationsbericht der Bundesregierung 2015.

BAMF, Aktuelle Zahlen zu Asyl, Ausgabe 08/2017, abrufbar unter www.bamf.de/downloads.

BAMF, Das Bundesamt in Zahlen: Asyl, Migration und Integration, 2016.

Bundesvereinigung der Deutschen Arbeitgeberverbände (BDA), Fachkräftezuwanderung erleichtern - Vier-Punkte-Plan, Stellungnahme vom 26.4.2017, abgerufen unter www.arbeitgeber.de>Beschäftigung.

Bünte, Rudolf/Knödler, Christoph, Einwanderungsgesetz: Plädoyer für weitere Ausdifferenzierung, in: ZRP 2018, S. 102-106.

Crawley, Heaven/Sklepari, Dimitris, Refugees, migrants, neither, both: categorical fetishism and the politics of bounding in Europe's 'migration crisis', in: Journal of Ethnic and Migration Studies, Vol. 44, S. 48-64.

de Haas, Hein/Natter, Katharina/Vezzoli, Simona, Growing Restrictiveness or Changing Selection? The Nature and Evolution of Migration Policies, in: International Migration Review 2016.

Dörig, Harald, Fachkräftegewinnung mit Blue Card oder Punktesystem, in: NVwZ 2016, S. 1033-1039.

Eurostat, Statistik: „Erstmals erteilte Aufenthaltstitel nach dem Grund für die Erteilung“, unter ec.europa.eu/eurostat, Code tps 00170 abgerufen am 5.3.2018 (zitiert als: Eurostat, Aufenthaltsstatistik).

Frontex, Risk Analysis for 2016.

Herzog-Schmidt, Julia, Die Blue-Card Richtlinie 2009/50/EG und ihre Umsetzung in Deutschland, 2014.

Hofmann, Rainer, Kommentar zum Ausländerrecht, 2. Auflage 2016 (zitiert als: Bearbeiter, in: Hofmann, Ausländerrecht, § Rn.).

Hörich, Carsten, Einwanderungsgesetzentwurf der Grünen, in ZRP 2017, S. 154. 
Hunger, Uwe/Krannich, Sascha, Einwanderung neu gestalten - Transparent, Attraktiv, Einfach, in: WISO Diskurs, 05/2017.

International Organization for Migration, Fatal Journeys, Volume 3 Part 1: Improving Data on Missing Migrants, 2017.

Klaus, Sebastian, Die Hochqualifizierten-Richtlinie 2.0: Geplante Novellierung der europarechtlichen Vorgaben zur Blauen Karte EU, in: ZAR 2017, S. 60-73.

Kluth, Winfried, Einwanderungsgesetz: Entwürfe - Chancen - Kritik, in: NVwZ 2018, S. 1437-1442.

Kluth, Winfried / Heusch, Andreas, Kommentar zum Ausländerrecht, 2016 (zitiert als: Bearbeiter, in: Kluth/Heusch, Ausländerrecht, § Rn.).

Kolb, Holger/Lehner, Roman, Radikale Moderne? Zum Entwurf eines Einwanderungsgesetzes (EinwG-E) der Bundestagsfraktion von Bündnis 90/Die Grünen, in: ZAR 2017, S. 270-277.

Kolb, Holger/Lehner, Roman, Vorschlag zu einem Einwanderungsgesetz Viel Lärm um wenig, in: ZRP 2017, S.34-37.

Kopp, Judith, Externalisierung der Verantwortung: Grenz- und AlibiFlüchtlingsschutz außerhalb Europas, abgerufen am 7.3.2018 unter https://www.proasyl.de/hintergrund.

Langenfeld, Christine, Tausche Arbeit gegen Asyl - Ein integrations- und arbeitsmarktpolitisch riskanter Weg, in: ZRP 2015, S. 193.

Maor, Oliver, Einreise und Aufenthalt von Geschäftsreisen-den, konzernintern Versetzten und anderen vorübergehend erwerbstätigen Ausländern - ein (fast) vergessener Teil der Wirtschaftsmigration, in: ZAR 2005, S. 333-340.

Offer, Bettina, Der Entwurf der SPD-Bundestagsfraktion für ein Einwanderungsgesetz - Analyse und kritische Stellungnahme, in: ZAR 2017, S. 29-34.

Organization for Eco-nomic Cooperation and Development (OECD), International Migration Outlook, Annual Re-port, 2007.

Pro Asyl, Balkanroute: Eine Chronik der Abschottung, abgerufen am 9.3.2018 unter www.proasyl.de/hintergrund/balkanroute.

Sachverständigenrat Deutscher Stiftungen für Migration und Integration (SVR), Chancen in der Krise: Zur Zukunft er Flüchtlingspolitik in Deutschland und Europa, Jahresgutachten 2017.

SVR, Deutschlands Wandel zum modernen Einwanderungsland, Jahresgutachten 2014. 
SPD-Bundestagsfraktion, Entwurf eines Einwanderungsgesetzes, Stand 7.11.2016, abrufbar unter www.spdfraktion.de/einwanderungsgesetz (zitiert als: EinwG-E (SPD)).

Strunden, Martin/Schubert, Michaela, Deutschland gibt sich Blue Card „Plus“ - EU-Richtlinie genutzt für Meilenstein der Arbeits-migration, ZAR 2012, S. 270-276.

Thym, Daniel, Einwanderungsgesetzgebung: Chancen und Illusionen (Teil 1), in: ZAR 2017, S. 297-304.

Thym, Daniel, Migrationssteuerung im Einklang mit den Menschenrechten - Anmerkungen zu den migrationspolitischen Diskursen der Gegenwart, in: ZAR 2018, S. 193-200.

United Nations High Commissioner for Refugees (UNHCR), Refugee Protection and Mixed Migration: A 10-Point Plan of Action, 2007.

Wergin, Clemens, „Deutschland hat das dümmste Einwanderungsgesetz überhaupt", in Die WELT vom 5.9.2015 unter www.welt.de/article 146042330/ abgerufen am 5.3.2018.

Wissenschaftlicher Dienst des Deutschen Bundestages, Änderungen des Asyl- und Aufenthaltsrechts seit Januar 2015 mit den Schwerpunkten Asylpaket I und II, WD 3, 018/16, 2016. 



\title{
Eine soziohistorische Perspektive auf Deutschlands Migrationsreformen
}

\author{
Un-/erwünschte Mobilität und die Debatte \\ um ,Wirtschaftsflüchtlinge‘
}

Merve Kania

\section{EINLEITUNG}

Die deutsche Bundesregierung hat sich 2018 auf die Verabschiedung eines Fachkräfteeinwanderungsgesetzes geeinigt. Dieses löst das sog. ,Zuwanderungsgesetz' aus dem Jahre 2005 ab und knüpft diskursiv an Positionen und Argumentationslinien in früheren Debatten zu den Reformierungen von Ausländergesetzen an. Bündnis 90/Die Grünen sowie die Sozialdemokratische Partei Deutschlands (SPD) schlugen beispielsweise schon in den Jahren 1990-1993 und 2001-2004 die Einführung eines ,Einwanderungsgesetzes` vor. Dem gegenüber sprach sich die CDU/CSU-Fraktion von ca. 20152018 für die Reformierung des seit bestehenden ,Aufenthaltsgesetzes ‘ zu einem ,Fachkräftezuwanderungsgesetz ‘ aus (Angenendt, Kipp, und Meier 2017, 9; Beeger und Schwenn 2018). Der Unterschied der beiden Vorschläge bestand nicht nur sprachlich sondern vor allem semantisch darin, was für eine Gesellschaft Deutschland sein soll und möchte und welche Personengruppen Deutschland dementsprechend für dieses Gesellschaftsbild attrahieren möchte. ${ }^{1}$ Dieser Beitrag zeichnet frühere Phasen und Debat-

1 Für akademische Diskussionen und Empfehlungen im Rahmen der Debatten zur Einführung eines Fachkräfte-/Einwanderungsgesetzes, siehe z.B. Uwe Hunger und Sascha Krannich 2017 sowie Eichenhofer und Hörich 2018. Für eine Dis- 
ten der deutschen Migrationsgeschichte nach und zeigt, dass sich eine prinzipielle Grundhaltung zur Unterscheidung in un-/erwünschte Migrationsformen schon in den 1970er und 1980er Jahren herausbildete.

2017 analysierten das Sozialforschungsinstitut Infratest Dimap sowie die Bundeszentrale für politische Bildung, dass „Flucht und Migration“ sowie „Zuwanderung von Flüchtlingen“ zu den zentralen Fragen an die Politik sowie den ausschlaggebendsten Themen für die Wahlentscheidung der stimmberechtigten Deutschen in der Wahl zum 19. Bundestag zählten (Hanewinkel 2017; Infratest dimap 2017). Dabei bildeten die sehr unterschiedlichen Migrationsformen ,Zuwanderung', ,Einwanderung ${ }^{6}$ und ,Asyl ${ }^{\circ}$ diskursiv ein Amalgam, welches sich so auch in früheren Debatten um ,Migrationsrecht' erkennen lässt. ${ }^{3}$ Dieser Beitrag versucht zu zeigen, dass diesem Diskurs einerseits eine Frage des Umgangs mit ,Ausländern“ per se als auch ,das Wohl' des Wohlfahrtsstaats, d.h. der deutschen Arbeits- und Sozialsysteme, als Gradmesser für un-/erwünschte Migrationsformen und entsprechende Zugangs- und Integrationsrechte zugrunde liegt.

Im Folgenden wird zunächst ein historischer Überblick über die phasenhafte Entwicklung der deutschen Migrationspolitik gegeben. AnschlieBend wird beispielhaft die (rhetorische) Figur des ,Wirtschaftsflüchtlings“ eingeführt, um an ihr die zunehmende Regulierung und Verengung der $\mathrm{Zu}$ gangsmöglichkeiten von AusländerInnen bzw. ,Versicherheitlichung ' der Migrationsdebatten und des Wohlfahrtsstaats aufzuzeigen. Diese Versicherheitlichung bildete sich einerseits schon innerhalb der 1970er und

kussion des Spannungsfelds von Zugehörigkeit und Recht, siehe die Beiträge von Kentaro Inagaki und Lise Känner in diesem Band.

2 In diesem Beitrag meine ich mit, Einwanderung ' den Prozess des sich intendierten unbeschränkten Niederlassens oder der Einbürgerung in ein Land (Immigration), mit ,Zuwanderung' den Prozess des sich auf nur beschränkte Zeit Niederlassens in einem Land, mit ,Flucht' das forcierte Verlassen einer Heimat aufgrund einer Gefahr für Leib und Leben - welches sowohl gesetzlich anerkannte als auch abgelehnte Beweggründe mit einschließt. Für eine juristische Definition und Diskussion dieser Begrifflichkeiten, siehe den Beitrag von Nils Imgarten in diesem Band.

3 Ähnlich stellten Anastasia Tsoukala (2011) im Falle von Frankreich und Helen O’Nions (2014) sowie James Hampshire (2011) im Falle von Großbritannien eine Zusammenführung von Mobilitätskanälen im öffentlichen Diskurs fest. 
1980er Jahre heraus und wurde andererseits auch von Politikern, wie etwa in den 1990er Jahren, durch das schüren von gesellschaftlichen Ängsten um den Missbrauch der deutschen Wohlfahrtsysteme (teilweise kontrafaktisch) geschürt. Dabei werden Sekundärliteratur, Bundesdruck-sachen, staatliche Berichte und Statistiken sowie Medienartikel diskutiert und analysiert. Abschließend werden die Ergebnisse zusammengefasst sowie Empfehlungen unterbreitet, wie angesichts der Persistenz des Arguments der Ausnutzung des Wohlfahrtsstaats durch einige Personengruppen und der sozioökonomisch artikulierten Notwendigkeit, zusätzliche Arbeitskräfte für die deutschen Wirtschafts- und Sozialsicherungssysteme zu attrahieren, ${ }^{4}$ an einen migrations- und identitätspolitischen Normenwandel in Deutschland herangegangen werden muss.

\section{DEUTSCHLANDS VERNEINENDE AUSLÄNDERPOLITIK}

Bis zum rot-grünen Koalitionsvertrag 1998, der anschließenden Reform des Staatsangehörigkeitsgesetzes 1999 sowie der Diskussion des ,Zuwanderungsgesetzes' durch die rot-grüne Koalition ab den Jahren 2001/2 hat jede deutsche Regierung versucht, die Illusion aufrecht zu erhalten, dass die Bundesrepublik kein Einwanderungsland, sondern ein Land mit homogener Bevölkerung sei (Oberndörfer 1994, 35; Deutscher Bundestag 2000, XIV). Im europäischen Vergleich war dies zu den damaligen Zeitpunkten zwar nicht untypisch, da sich mit Ausnahme Großbritanniens lange Zeit keines als „Einwanderungsland“ bezeichnete (Constant und Tien 2011, 6; Mehrländer 1987). 1999 war Deutschland allerdings das letzte westeuropäische Land, welches neben dem jus sanguinis ein beschränktes jus soli für die Nachkommen von in Deutschland lebenden Ausländern im ,Staatsangehörigkeitsgesetz' ergänzte. De facto, war die Geschichte der Bundesrepublik seit ihrer Gründung 1949 immer von ,Migration“ geprägt; laut Klaus J. Bade (2015; 2017a; 1994) ist deutsche Geschichte gar Migrationsgeschichte. Mit insgesamt 44 Millionen Zugewanderten sowie 32 Millionen Deutschen wie Nichtdeutschen Ausgewanderten zwischen 1950 und 2014

4 Für eine intensivere Diskussion der wirtschaftspolitischen Aspekte, siehe den Beitrag von Anna Hochleitner in diesem Band. 
(Beauftragte der Bundesregierung für Migration 2017, 16) und schwarzen Zahlen in der Zuwanderung seit 1957 war Deutschland dies jedoch schon lange.

Damit soll nicht ausgedrückt werden, dass die Bundesrepublik die Mobilitätsbestimmungen, Rechte und Pflichten von Ausländern nicht reguliert hätte. Sondern eher, dass bis in die 1990er Jahre Fragen einer langfristigen Konzeption der Integration von GastarbeiterInnen, ihren Familien und Geflüchteten zugunsten einer arbeitsmarktorientierten Migrationspolitik gekoppelt mit einer Politik der Abwehr von potentiellen Ausnutzern des Wohlfahrtstaats vermieden wurden (Deutscher Bundestag 2000, 39; Bade 2015, 5; Roman Eckendorff und Markus Stock 2016, 5; vgl. Bade 1994). So teilt etwa der sechste Familienbericht: Familien ausländischer Herkunft in Deutschland: Leistungen - Belastungen - Herausforderungen (Deutscher Bundestag 2000) der Bundesregierung ,Migration“ (hier i.S.v. ,nachfrageorientierter Arbeitsmigration') als Teil von ,Ausländerpolitik entlang juristischer Meilensteine in fünf Phasen ein, von denen die ersten drei in diesem Abschnitt überblicksartig vorgestellt werden bevor die anschließenden Phasen in Kombination mit den dazugehörigen migrationspolitischen Debatten im weiteren Verlauf dieses Beitrags diskutiert werden:

Die erste Phase („Anwerbeperiode“ bzw. "Gastarbeiterperiode“) begann 1955 mit der Anwerbung ausländischer Arbeitskräfte für die Gastarbeiterprogramme, führte zur Verabschiedung des ersten Ausländergesetzes 1965, welches die dazugehörigen Verwaltungsvorschriften und Aufenthaltserlaub-nisse regulierte und mündete in der Einführung des Anwerbestopps anlässlich des „Ölpreisschocks“ 1973 sowie steigender Arbeitslosigkeit unter Deutschen wie Gastarbeitern und ihren Familien (ebd., 37-38; Bade 2015, 4). Als „beschäftigte Ausländer (...) als unerwünschte Konkurrenten am Arbeitsmarkt (...) [und] arbeitslose ,Gastarbeiter ' als soziale ,Kostgänger' diskreditiert wurden“ (ebd.), wurde die staatlich organisierte Arbeitsmigration vorerst beendet ${ }^{5}$ und nur noch direkte Familienangehöri-

5 Walter Arendt, Bundesminister für Arbeit und Soziales, schrieb darin am 23.11.1973: „Es ist nicht auszuschließen, dass die gegenwärtige Energiekrise die Beschäftigungssituation in der Bundesrepublik Deutschland in den kommenden Monaten ungünstig beeinflussen wird. Unter diesen Umständen ist es nicht vertretbar, gegenwärtig weitere ausländische Arbeitnehmer über die Auslands- 
ge, d.h. Ehegatten und Kinder, sowie Personen, die in einem der ausgeschriebenen Mangelberufe ausgebildet waren mit einer Aufenthaltserlaubnis ausgestattet (Butterwegge 2005).

In der darauffolgenden sog. zweiten Phase der Ausländerpolitik (bzw. „Phase der Konsolidierung der Ausländerbeschäftigung“) wurde von 1973 bis 1979 ein Schwerpunkt auf die „Eingliederungspolitik für diejenigen Familien angekündigt, die sich längerfristig in der Bundesrepublik Deutschland niederlassen wollten“ (Deutscher Bundestag 2000, 38). Allerdings waren deren Aufenthaltserlaubnisse grundsätzlich unter dem Vorbehalt erteilt worden, dass ihre Anwesenheit ,die Belange der Bundesrepublik Deutschland (§2 AuslG 1965) nicht beeinträchtigen würden“ (ebd.). Bis zur „Reform des Ausländerrechts 1990/91 [...] war gesetzlich nicht geregelt, unter welchen Bedingungen eine Aufenthaltsverlängerung genehmigt bzw. verweigert werden konnte“ (ebd.). Gleichzeitig wurde von 1975-77 für die sogenannten „überlasteten Siedlungsgebiete“ Hessen, Nordrhein-Westfalen, Bayern, Baden-Württemberg und Berlin eine ,Zuzugssperre“ für den Familiennachzug verhängt (Hanewinkel 2017), die jedoch in der Praxis schwer durchzusetzen war und von wirtschaftlichen Kräften kritisiert wurde (Deutscher Bundestag 2000, 39).

Als der erste Ausländerbeauftragte der Bundesregierung, Heinz Kühn (SPD), der damals dem Bundesarbeitsministerium zugeordnet war, sein Memorandum Stand und Weiterentwicklung der Integration der ausländischen Arbeitnehmer und ihrer Familien in der Bundesrepublik Deutschland vorlegte, wurde laut dem o.g. Familienbericht die dritte und kürzeste Phase („Phase der konkurrierenden Integrationskonzepte“ 1979-1980) eingeleitet (ebd.; vgl. Butterwegge 2005). Das auch als Kühn Memorandum bekannte Schreiben forderte die „Anerkennung der faktischen Einwanderungssituation $[, \ldots]$ eine konsequente Integrationspolitik und damit eine Abkehr von der Priorität arbeitsmarktpolitischer Gesichtspunkte (ebd., 39). Zudem führte es zur Produktion eines konkurrierenden Papiers des Koordinierungskreises ausländische Arbeitnehmer seitens des Bundesarbeitsministeriums. Ungeachtet beider Vorschläge hielt die damalige Bundesregierung in ihren Beschlüssen vom März 1980 jedoch ,,an einer durch Konzepte zur ,sozialen Integration auf Zeit‘ ergänzten Ausländerpolitik fest““(ebd., 40.).

dienststellen der Bundesanstalt für Arbeit für eine Arbeitsaufnahme in der Bundesrepublik zu vermitteln“(BPB 2011). 


\section{DER ,WIRTSCHAFTSFLÜCHTLING‘ IN DEN BUNDESTAGSDEBATTEN}

Die offizielle Version der Bundeszentrale für politische Bildung (Butterwegge 2005) und des Familienberichts der Bundesregierung (Deutscher Bundestag 2000) bezeichnet die anschließende vierte Phase (1981-1990) offiziell als „Wende in der Ausländerpolitik“. In diesem Abschnitt möchte ich aufbauend auf den Werken von Klaus J. Bade (2015; 2017a) sowie eigenen, weiterführenden Recherchen in den Bundesdrucksachen die Rolle von ,Wirtschaftsflüchtlingen“ für den migrationspolitischen Diskurs anführen. Hierzu ist der Bedeutungswandel des Begriffs und der Kontext sowie die Häufung seiner Benutzung herauszustellen. ${ }^{6}$ Während des zweiten Weltkriegs bezeichnete, Wirtschaftsflüchtling‘ aus Deutschland flüchtende und in anderen europäischen Staaten schutzsuchende Juden. Wenige Monate nach den beiden deutschen Staatsgründungen ab dem Winter 1949/50 als „Abstimmung mit den Füßen zugunsten des Westens“ (Bade 2015, 3) politisch begrüßte Ost-West-Migration. In den Bundestagsdebatten (ab 1949) wurde die Figur des ,Wirtschaftsflüchtlings' allerdings erstmalig zwischen November 1965 und Februar 1966 vom CDU Abgeordneten Dietrich Rollmann, insgesamt sechs Mal für Geflüchtete aus den Ostblockstaaten (z.B. Ungarn) im Kontext von Diskussionen des 1965er Ausländergesetzes, verwendet.

Da der Begriff anschließend bis ins Jahr 1978 nicht genutzt wurde, geht Klaus J. Bade (2015) davon aus, dass sich diese Färbung des Begriffes nicht etabliert habe. Eine Analyse der Häufung des Begriffs in den Bundesdrucksachen bestätigt dies. Ab 1978 wurde ,Wirtschaftsflüchtling' (151 aus insgesamt 157 Treffern) in jedem Jahr außer 2005 und 2010 genutzt sowie interessanter Weise diskursiv ab 1979 von seinen Ablegern dem ,Scheinasylanten“ (66 Treffer), ab 1981 vom ,Armutsflüchtling‘ (69 Treffer) und ab 1991 vom ,Sozialschmarotzer‘ (18 Treffer) begleitet. Dies lässt sich rein quantitativ betrachtet als Ausdruck einer zunehmenden Politisierung und Unerwünschtheit von gewissen Migrationsformen ab der erwähnten „,dritten Ausländerphase" deuten und wird in den nachstehenden Abschnitten dieses Beitrags in einen näheren migrationspolitischen Kontext gestellt.

6 Die entsprechenden Datenbankabrufe der Bundesdrucksachen (ab 1949) erfolgten am 16.11.2018. Siehe: http://pdok.bundestag.de/. 
Semantisch betrachtet geben die Eigenschaften, die einem, Wirtschaftsflüchtling' nachgesagt werden, Aufschluss über tiefliegende Ängste, die Politiker im deutschen Diskurs adressieren und ggf. aufgrund gesellschaftlichen Unbehagens (vgl. Folgeabschnitte) auch adressieren müssen. Hierzu zählt vor allem das untersagte Vortäuschen von politischen Verfolgungsgründen um über einen Asylstatus Zugang zum deutschen Wohlfahrtssystems, d.h. Arbeitsplätzen und sozialen Sicherungssystemen, zu erhalten. Beispielsweise führte der CDU/CSU Abgeordnete Carl-Dieter Spranger 1978 während der Beratung des von den Fraktionen der SPD und FDP eingebrachten Entwurfs zur Beschleunigung des Asylverfahrensgesetzes den Anstieg der Asylbewerberzahlen von 5.900 (1973) auf 16.410 (1977) gegenüber sinkenden Anerkennungszahlen darauf zurück, ,dass Asyl in steigendem Maße missbräuchlich verlangt [worden sei]. Die weit überwiegende Zahl der heutigen Asylbewerber [schütze] lediglich persönliche politische Verfolgung vor, um sich wirtschaftliche Vorteile durch langjährigen Aufenthalt in der Bundesrepublik Deutschland zu verschaffen“ (Deutscher Bundestag 2017, 7370).

Bezeichnend ist, dass in allen Dekaden die Debatten um „Asylmissbrauch" von Wirtschaftsflüchtlingen auch bei sinkenden Asylbewerberzahlen (Bade 2015, 4, 6; vgl. Müller 2010, 39), vornehmlich von Seiten der CDU/CSU und auch bei hohen Gesamtschutzquoten geführt wurden. In dem Zeitraum 1990-2017, für den Statistiken des Bundesamts für Migration und Flüchtlinge (BAMF) öffentlich zugänglich sind, wurde der Begriff ,Wirtschaftsflüchtling' trotz einer Gesamtschutzquote von ca. jedem zehnten 1999 zwei Mal, bei einer Anerkennungsquote von noch nicht einmal jedem hundertsten $(0,9 \%)$ im Jahre 2005 kein einziges Mal, dafür aber bei einer Gesamtschutzquote von jedem zweiten bzw. zwei von dreien in den Jahren 2015-16 15 Mal verwendet (Angenendt, Kipp, and Meier 2017, 9; BAMF 2005, 57). Zusammengefasst sind ,Wirtschaftsflüchtlinge' also während aller Perioden vor allem eine rhetorische Figur, die für ,unerwünschte', d.h. in diesem Fall das deutsche Flüchtlingsrecht und den Wohlfahrtsstaat ausnutzende, Migration im Diskurs steht. 


\section{ZUR VERSICHERHEITLICHUNG DER MIGRATIONS-DEBATTEN}

Die Verwendung und Häufung der rhetorischen Figur des ,Wirtschaftsflüchtlings‘ hängt unmittelbar mit der Führung von Debatten um die Reformierung des Migrationsrecht, d.h. sowohl des Asyl- als auch des $\mathrm{Zu}-$ wanderungsrechts, zusammen. In den 20 Jahren von 1978 bis 1998 gab es 93 Verwendungen, von denen 78 und somit 49,7 Prozent aller Wortnennungen zwischen Juni 1978 und Juli 1993, als die Änderung des Grundrechts auf Asyl (Art. 16a GG) in Kraft trat, liegen. Nachdem die Asylbewerberzahlen von 1987 bis 1992 kontinuierlich von 103.076 auf 438.191 anstiegen (BAMF 2017, 9) wurde der sog. ,Asylkompromiss' in der fünften Phase der Ausländerpolitik ausgehandelt (Deutscher Bundestag 2000, 42). Anstatt ein separates ,Flüchtlingsgesetz' und ein ,Einwanderungsgesetz einzuführen, wie Anfang der 1990er Jahre Bündnis 90/die Grünen und die SPD vorschlugen, wurde der Asylparagraph (Art. 16 bzw. 16a GG) verengt. Für Personen, die aus einem sog. ,sicheren Drittstaat' oder einem Mitgliedsland der Europäischen Gemeinschaft (bzw. Union) eingereist waren, wurde prinzipiell die Möglichkeit, ein individuelles Verfolgungsmerkmal zu tragen, nicht angenommen (Deutscher Bundestag 1992; 1993). Dies reduzierte die Asylbewerberzahlen sowohl aufgrund der Zuständigkeiten, die sich wegen des Faktors der ,(Erst-)Einreise' an die EU-Außengrenzen verschob, als auch aufgrund der Herkunft der Bewerber, da viele europäische Anrainerstaaten als ,sicher' klassifiziert wurden.

Interessant ist hierbei auch, dass 40 der 78 Nennungen in den Zeitraum von Anfang 1978 bis Ende 1987 fallen, wobei keine Anerkennungsquoten zur näheren Einschätzung dieser Werte vorliegen (BAMF 2005). Klaus J. Bade $(2015,6)$ argumentiert in diesem Kontext, dass die Debatten um die Verhinderung von Asylmissbrauch in den 1980er Jahren gar derart lautstark geführt worden seien, dass sie von den Konzeptionsschwierigkeiten in der Integration von der sich bereits im Inland befindenden ,Ausländerbevölkerung' bzw. der ausländischen (Gast-) Arbeitnehmer und ihrer Familien ablenkte. Gleichzeitig hätten die Asylbewerberzahlen seit dem ,Anwerbestopp، von 1973 zur Zuwanderung nach Deutschland einen enormen Anstieg erlebt: Zum Einen bot der Anwerbestopp im Zusammenspiel mit den sog. ,Ausnahmeverordnungen“ nur ein „Nadelöhr“ (ebd., 9), welches nicht ausreichend bzw. nicht der realen Nachfrage entsprechend Möglichkeiten 
zur legalen Zuwanderung nach Deutschland eröffnete. Zum Anderen war (und ist) eben jene Nachfrage im Zuge globalpolitischer Veränderungen und Kriegen strukturell bedingt (ebd., 4). ${ }^{7}$

In der Annahme, dass Asylbewerber und Wirtschaftsflüchtlinge aufgrund von wohlfahrtsorientierten „Fluchtanreizen“ Deutschland auswählten, wurden seit den 1970er Jahren Maßnahmen zur ,Erschwerung der Einreise von Flüchtlingen und Asylsuchenden, Beschleunigung der Asylverfahren und Verschlechterung der Lebensbedingungen von Asylbewerbern“ (ebd., 5) eingeführt, die in den Bundesländern unterschiedlich umgesetzt wurden. ${ }^{8}$ Des Weiteren wurde das ,Asylverfahrensgesetz' (seit dem 24. Oktober 2015 unbenannt in Asylgesetz) mehrmals geändert. In diesem $\mathrm{Zu}$ sammenhang wurde zwar ,der Familiennachzug sowie die Einbürgerung erleichtert (...), [der] Schutz von Eherpartner(inne)n und Kindern politisch Verfolgter [ausgedehnt] und eine sog. Altfallregelung für geduldete Asylbewerber/innen [eingeführt], d.h. ihnen wurde ein dauerhaftes Aufenthaltsrecht zugestanden. Gleichzeitig wurden jedoch die Ausweisungsbefugnisse (z.B. bei Straftaten) verschärft und die Ermessensspielräume der Ausländerbehörden im Aufenthaltsrecht ausgeweitet" (Reißlandt 2002, 218). Kurz gesagt, der sogenannte ,Asylkompromiss‘ gab, in den Worten des Bundestagsabgeordneten Klaus Heiner Lehne (MdB, CDU) ,die Möglichkeit, endlich wirksam zwischen politisch Verfolgten und Wirtschaftsflüchtlingen zu unterscheiden“ (Deutscher Bundestag 1993b, 13573).

Unterstützend trat 1993 das Asylbewerberleistungsgesetz in Kraft, welches die Höhe und Form von Leistungen für Asylbewerber, Geduldete und Abschiebeschutztragende in Angrenzung an ihre Aufenthaltsstatus bis 2012 unangepasst festlegte. ${ }^{9}$ Während Klaus J. Bade (2015, 5; vgl. Reißlandt

7 Die OECD $(2016,16)$ geht in ihrem Bericht „States of Fragility“ davon aus, dass sich zwar die Anzahl der Menschen, die in extremer Armut leben verringern, aber die derjenigen die der extrem armen in fragilen Kontexten von 480 Mio. (2015) auf 542 Mio. (2035) steigen werde - was aus Sicht der klassischen Migrationswissenschaften als push-Faktor gilt.

8 Beispielhaft sei hier das „Gesetz zur Beschleunigung des Asylverfahrens“ vom 1. August 1978 genannt.

9 Das Bundesverfassungsgericht befand am 18. Juli 2012, dass die Höhe der Geldleistungen nach $\S 4$ Asylbewerberleistungsgesetz unzureichend sei, weil diese einerseits seit 1993 nicht vom Gesetzgeber verändert wurden und diese 
2002, 220) diese Entwicklungen nicht zuletzt aufgrund der DublinVerordnungen im Rahmen der Europäischen Gemeinschaft bzw. Union als „Konsens der Abwehr“ sieht und Ole Waever sowie Barry Buzan (1993) von der Herausbildung eines Problems der Sicherung der Identität einer Gesellschaft (societal security) sprechen, zeigt sich diese Dynamik im Zuge der in diesem Beitrag diskutierten Asyl- und Zuwanderungsdebatten in Form eines dem vorangehenden und zunehmend weiter versicherheitlichten Wohlfahrtsstaats in Form von prozeduralen und sozioökonomischen Möglichkeiten und Rechten.

Nach der Reformierung des Staatsangehörigkeitsrechts 1999, Empfehlungen von Expertenkommissionen, Drängen seitens wirtschaftlicher Kräfte sowie intensiven Debatten in den zwei politischen Kammern kodifizierte das sog. ,Zuwanderungsgesetz' 2005 letztendlich nur, was de facto schon seit den Gastarbeiterprogrammen, dem Anwerbestopp und Ausnahmeverordnungen der Fall war: Dass Deutschland die Zuwanderung von ausländischen Personen nicht generell erwünscht, sondern gemäß seiner sozioökonomischen Interessen reguliert. So wurde bereits im ersten Paragraphen mit dem Titel ,Zweck des Gesetzes; Anwendungsbereich“ (§ 1 Abs. 1 ZuWG, $\S 1$ AufenthG) festgelegt, dass Zuwanderung von Personen nur unter der Berücksichtigung von konkreten Nachfragen am Arbeitsmarkt sowie einem nicht weiter definierten Kriterium der ,Aufnahme- und Integrationsfähigkeit' dieser Personen gestattet werden solle. Laut Jan Schneider (2007) wurde der Gesetzestext deshalb dafür kritisiert, dass er nicht die ,Öffnung ${ }^{6}$ bringe, die der oft verwendete Kurztitel ,Zuwanderungsgesetz' suggeriere. Sein voller Titel Gesetz zur Steuerung und Begrenzung der Zuwanderung und zur Regelung des Aufenthalts und der Integration von Unionsbürgern und Ausländern 2001/2 erschien hier aussagekräftiger. Müller (2012, 25) resümiert etwa, dass diese Anforderungen an die Förderung und Begrenzung von Zuwanderung unweigerlich in einer selektiven Migrationspolitik münden, „da die (stillschweigende) Zustimmung der Bevölkerung zur Öffnung des Arbeitsmarkts durch die Schließung anderer Migrationskanäle,

andererseits unter Berücksichtigung des im Grundgesetz verankerten Menschenrechts auf menschenwürdiges Leben (Art. 1 Abs. 1 GG) in Verbindung mit dem Sozialstaatsprinzip (Art. 20 Abs. 1 GG) nicht an den Aufenthaltsstatus einer Person gekoppelt werden dürften (siehe BVerfG 2012). 
etwa beim Familienzuzug oder bei der Aufnahme Asylsuchender erkauft werden muss."

\section{FREMDENFEINDLICHKEIT, ABSCHIRMUNG UND POLITISCHE REPUTATION}

Während man annehmen könnte, dass ein Verlangen nach z.B. ,societal security“ bottom-up von der deutschen Bevölkerung an die Politik getragen wurde, macht Klaus J. Bade (2017b; 2015b) deutlich, dass durch die Benutzung von Sprache, Fremdenfeindlichkeit und -skepsis in den 1990er Jahren auch von der Politik top-down geschaffen wurden. Bade nennt dies eine Flucht der Politik aus der eigenen Planlosigkeit ,,in Angst vor dem Bürger als Wähler" (Bade 2017a, 89). In den Reden von Politikern seien die „Grenzen der Belastbarkeit durch Zuwanderung“ immer sehr schnell erreicht oder in den Worten des ehemaligen sozialdemokratischen Innenministers Otto Schily 1999 ,überschritten“ gewesen (Bade 2017b). Diese beruhten auf Angst, die oft recht zynisch grundiert war, nicht nur beim Sozialdemokraten Schily, der sich Jahre später für seine Falschmeldung zu den angeblichen Grenzen der Belastbarkeit mit den Worten, ,Ich wollte damals ein wenig mein rechtes Profil schärfen“ (zitiert nach Bade 2017b), rechtfertigte. Letztendlich hat jedoch insbesondere die CDU/CSU Asylmissbrauch als „Einwanderung in die Sozialsysteme“ und einen Zustand, der beendet werden müsse, weil sonst der innere Friede gefährdet sei, dargestellt (Thränhardt 2009, 47; CDU and CSU 2002), was ggf. Ansatzpunkte für historische Populismusforschung bietet.

In den 1990er Jahren sollen Anti-Asyl-Ressentiments innerhalb der Bevölkerung mittels Leitfäden und Kampagnen von der CDU/CSU gezielt angestachelt worden sein (Bade 2015, 6; Müller 2010, 39). ${ }^{10}$ Als gewaltbereite Minderheiten Asylwohnheime (Hoyerswerda 1991, Rostock Lichtenhagen 1992, etc.) anzündeten und Mobs auf die Straße gingen, stieß die wachsende „Bürgerangst und Bürgerwut von ,unten“ auf die anhaltende Konzeptionslosigkeit von ,oben“" (Bade 2017b, 494; vgl. Unwort des Jahres 1992

10 Siehe Walter Jakobs, 1991, „CDU plante die Antiasyldebatte“, taz.die tageszeitung, 8. Oktober 1991, Düsseldorf: http://www.taz.de/Archiv-Suche/!1699589\& $\mathrm{s}=\&$ SuchRahmen $=$ Print $/$. 
„Politikverdrossenheit“). Dies lieferte weltweit einen Grund für Entsetzen und deutschlandweit eine weitere Legitimationsgrundlage zur erwähnten Einschränkung des Asylrechts im Zuge der Reformierung des Art. 16 bzw. 16a GG (Bade 2015, 6; Müller 2010, 39). Vor diesem Hintergrund muss man Bundeskanzlerin Angela Merkels Umgang und Rhetorik mit der Flüchtlingsfrage im Sommer 2015 als klaren Bruch mit ihrer Parteigeschichte sehen: Während der Bundeskanzler der 1990er, Helmuth Kohl, vom „Staatsnotstand“ sprach, weil sich die Parteien gegenseitig im Asylstreit lähmten (Bade 2015, 6), schritt Merkel mit einem „wir schaffen das“ voran (Die Bundesregierung 2015).

Des Weiteren wurden Kernbegrifflichkeiten des mobilitätspolitischen Amalgams ,Flucht' und ,Zuwanderung' immer wieder zahlentechnisch zusammengefasst, verwechselt oder vermischt. Beispielsweise wurde von Entscheidungsträgern in den Migrationsdebatten immer wieder vergessen, die Zuwanderungs- gegen die Abwanderungszahlen aufzurechnen oder neben Asyl nach Art. 16a GG die anderen drei Schutzstatus (Flüchtlingsschutz, subsidiären Schutz, Abschiebungsverbot) für die Gesamtschutzquote zu berücksichtigen (Bade 2017, 10). So behauptete der von 1998-2005 amtierende Bundesinnenminister Otto Schily 1999, dass jährlich etwa 100.000 Flüchtlinge nach Deutschland kämen, von denen nur drei Prozent asylwürdig und der Rest Wirtschaftsflüchtlinge seien (ebd.). De facto fielen 1999 jedoch 9,1 Prozent in die Gesamtschutzquote (Angenendt, Kipp, und Meier 2017, 9). Dabei bleibt zu beachten, dass die Akkuratheit von Zahlen und Vergleichswerten Auswirkungen auf den Verlauf von Debatten ${ }^{11}$ und der Umstand, welche Daten eine Statistik überhaupt erfasst, Rückschlüsse darauf zulässt, welche Fragen überhaupt von gesellschaftlicher Relevanz sind.

In diesem Kontext ist auch zu beachten, dass sich die deutsche, statistische Definition von denen anderer Länder und Organisationen unterscheidet. Zum Beispiel zählt das statistische Bundesamt in seiner „Wanderungsstatistik" jegliche Form von Migration auf das deutsche Territorium inklusive der Asylzahlen und auch sehr kurzer Bleibeformen wie Auslandsstu-

11 Siehe auch Christopher C. Leite und Can E. Mutlu, 2017, „The Social Life of Data: The Production of Political Facts in EU Policy Governance“, Global Governance: A Review of Multilateralism and International Organizations: January-March 2017, Vol. 23, No. 1, S. 71-82. 
dien, Sprachaufenthalte oder kurze Ausbildungs- und Arbeitsverhältnisse als ,Zuwanderung' (BAMF 2016). Dies steht in Diskrepanz zur Definition der Vereinten Nationen (VN), welche eine beabsichtigte Aufenthaltsdauer von mindestens einem Jahr als Grundlage nimmt. Somit wäre Deutschland im europäischen Vergleich zwar das Hauptzielland von Migration, was statistisch für seine Standortattraktivität spricht, allerdings läge die Anzahl der Zuzüge bspw. im Jahr 2014 bei einer Anwendung der VN Definition mit 884.893 anstatt 1.454 .724 um 40\% niedriger (ebd., 10). Dies betont abermals die Notwendigkeit, einen ganzheitlichen Ansatz, der sowohl Flucht als auch Arbeitsmigration berücksichtigt, in der Analyse der Migrationspolitik Deutschlands als (ehemalige), Ausländerpolitik` zu folgen. Gleichsam eröffnen sich weitere Möglichkeiten für die deutsche Migrationsforschung, mögliche Zusammenhänge und Wechselwirkungen etwa zwischen der seit 2015 wieder vermehrt auftretenden Anschläge gegen Asylheime und Busse mit Geflüchteten und Aussagen von Politikern zu untersuchen. ${ }^{12}$

\section{TRANS-/NATIONALE HERAUSFORDERUNG MIGRATION}

Seit der „Wende vom 20. zum 21. Jahrhundert [ist] Bewegung in die Diskussion um eine aktive Migrations- und Integrationspolitik gekommen“ (Butterwegge 2005). ,Mobilität' hat global zugenommen und sich als feste Währung (Bauman 1998; 2000) in einer wirtschaftlich interdependent vernetzten Welt etabliert. Laut der International Organisation on Migration (IOM) haben sich 2015244 Millionen Menschen außerhalb ihres Heimatlands aufgehalten (IOM 2016a); gar jede siebte Person weltweit lebte oder arbeitete außerhalb ihres Geburtsorts (IOM 2016b). Ebenso haben sich die Gründe, warum Menschen ihre Heimat verlassen, seit dem kalten Krieg, der Anpassung des Ausländergesetzes und dem Asylkompromiss 1991-93 sowie der Reformierung des Zuwanderungsgesetzes 2005 verändert: heimische Arbeitnehmer deck(t)en (erneut) nicht die ökonomischen und nun

12 Für eine Diskussion der Zusammenhänge von PEGIDA, AfD und deutscher Einwanderungspolitik, siehe Schammann 2018. 
auch zunehmend die demographischen Bedarfe des Landes. ${ }^{13}$ Zudem erhält ein Gros der Schutzstatusträger seinen Schutz anstelle von politischer Verfolgung (Asyl) aus humanitären Gründen (BAMF 2017, 36; Butterwegge 2005).

Nicht zuletzt vor diesen Hintergründen war und ist deutsche Migrationspolitik doppelten Erwartungen und Zwängen auf nationaler und europäischer Ebene ausgesetzt. James Hollifield $(2011,220 ; 2015,188)$ nennt das Phänomen, das Staaten von oben durch Globalisierung und von unten durch Multikulturalismus in Ihrer Stabilität(swahrnehmung) bedroht werden, ,liberales Paradox'. Einerseits verlangen sozioökonomische Kräfte nach ,Öffnung' von Migrationskanälen, was der Beitrag von Anna Hochleitner in diesem Band intensiver analysiert. Andererseits drängen, wie die vorangehend diskutierten Beispiele zeigen, nicht nur - wie Hollifield behauptet Wähler auf den ,Erhalt von Sicherheit` in Form einer nationalen Identität (vgl. Buzan 1993) sondern im Kontext von Flucht und Asyl auch Politiker. Bade (2017a, 89-96) argumentiert, dass letztere dieses täten, um ihre Wähler nicht zu verängstigen und dass, täten sie es nicht oder nicht genügend, populistische Debatten wie die 2010/11 um das Buch des ehemaligen SPDPolitikers Thilo Sarrazins, „Deutschland schafft sich ab“, die Mordserie des Nationalsozialistischen Untergrunds (NSU) die seit 2011 sukzessiv aufgedeckt wurde (und sich zwischen 1998-2011 zutrugen) stattfänden. Konkret werden in diesem Kontext „Ängste vor ökonomischer und sozialer Konkurrenz, gruppenpsychologische Abwehrmechanismen gegen ,Fremde“ und kulturelle Überfremdungsängste" (Oberndörfer 1994, 35; vgl. Foroutan 2018; Bröse 2018) genannt.

Zwischen 1991 und 2001 sprach sich die deutsche Bevölkerung mehr oder weniger konstant mit etwa $60 \%$ für eine „Einschränkung der Zuwanderungsmöglichkeiten aus, während etwa 20 Prozent für einen vollständigen Einwanderungsstopp votierten. Auch neuere Zahlen bestätigten diese Einschätzung [Heitmeyer, 2006, 2007]“ (Müller 2012, 25). Ähnlich gaben in der IOM $(2015,38)$ Umfrage How the World Views Migration 34 Prozent der befragten Deutschen an, dass Immigration verringert werden solle, 49 Prozent, dass es auf dem aktuellen Level bleiben und nur 14 Prozent, dass sie sich erhöhen müsste (2 Prozent antworteten „don't know / refused

13 Für eine intensivere Diskussion der wirtschaftspolitischen Aspekte, siehe den Beitrag von Anna Hochleitner in diesem Band. 
to answer"). Dieser Trend ist bei einer historisch bedingt eher homogen geprägten Bevölkerungsstruktur und auch im europäischen Vergleich nicht untypisch; 2015 gaben 48\% im europäischen Mittel an, dass sie Immigration gerne senken würden (IOM 2015, 41). Jonathan Chaloff und Georges Lemaitre (2009; cf. Hollifield 2015, 191) befanden in ihrer Studie für die OECD deshalb, dass Migrationsreformen in Ländern wie Deutschland seit der Einstellung der Gastarbeiterprogramme in den 1970er Jahren darauf abzielten, ,Zuwanderung' marktnachfrageorientiert zu beschränken.

Anstatt soziokultureller Maßnahmen nutzte Deutschland primär arbeitsmarktorientierte Methoden, um Zuwandernde in die Gesellschaft zu integrieren (Leggewie 2000; Takle 2007). Das heißt, ,der Wohlfahrtsstaat“ wurde nicht nur, versicherheitlicht' i.S.v. abgeschirmt, sondern auch als Gradmesser und Katalysator zur Teilhabe in Deutschland verwendet. So forderte im Jahre 2001 die sogenannte Süßmuth-Kommission einen grundlegenden Wandel in der deutschen Migrations- und Integrationspolitik und empfahl, ähnlich wie die SPD und Bündnis 90/die Grünen in den Jahren 1992-3 und 2015-18, u.a. die Einführung eines Punktesystems „über das Bewerberinnen und Bewerber nach verschiedenen Kriterien wie etwa Alter, Sprachkenntnisse und Ausbildung ausgewählt werden“ (ebd.). Im letztendlichen Entwurf des Zuwanderungsgesetzes aus dem Jahre 2005 wurden solche Aspekte weniger genutzt, sondern Migration eher - auch im Zuge von Anti-Terror-Maßnahmen nach dem 11. September - mit weiteren Verschärfungen zur Aufnahme von Asylsuchenden und ihrem „Rückkehrmanagement" versicherheitlicht (ebd.).

\section{DER WEG ZUM FACHKRÄFTE-/EINWANDERUNGS- GESETZ}

Nachdem im Mai 2014 der damalige Bundespräsident Joachim Gauck in seiner Rede zum 65. Jubiläum des deutschen Grundgesetzes für die Normalisierung eines ,neue[n] deutsche[n] ,Wir', [... als] die Einheit des Verschiedenen“ (Gauck 2014; vgl. Oltmer 2014) geworben und ,Einwanderung' als von und in Deutschland schließlich erkannte „Chance“ bezeichnet hatte, schlugen SPD und Bündnis 90/die Grünen abermals im Winter 2014/15 die Einführung eines Punktesystems zur Regulierung der Zuwanderung von Fachkräften nach Deutschland vor (Deutscher Bundestag 
2015). ${ }^{14}$ Obwohl CDU-Generalsekretär Peter Tauber zum Jahreswechsel zunächst selbst die Einführung eines Modells nach kanadischem oder australischem Vorbild gefordert hatte, änderte sich seine Rhetorik im Zuge von unionsinternen Debatten im Februar 2015 dahingehend, dass man nicht für „mehr Migration“ werbe (Simantke 2015), aber ggf. für eine bessere und widerspruchsfreie Verknüpfung der diversen bestehenden Regulierungen in den bereits bestehenden Gesetzestexten ${ }^{15}$ eintrete (Diekmann 2015).

Währenddessen betonten der damalige Bundesinnenminister Thomas de Maizière und CSU-Parteichef Horst Seehofer, dass Deutschland in Form seines Aufenthaltsgesetzes, welches aus der EU Blue Card, den Visumsregelungen zur Arbeitsplatzsuche für Hochqualifizierte sowie den Zuwanderungserlaubnissen zwecks der Aufnahme einer Ausbildung in einem der ausgewiesenen Mangelberufe bestünde, sowohl ein Einwanderungsgesetz als auch ein Punktesystem habe, bei dem „Bewerber aus Drittstaaten ... nur zwei Punkte erfüllen [müssten]: einen Hochschulabschluss und einen Arbeitsvertrag mit einem bestimmten Mindestgehalt" (FAZ 2015). Laut de Maizière müsste lediglich mehr Werbung dafür insbesondere seitens wirtschaftlicher Akteure gemacht werden (SPON 2015). Während des Anstiegs der Asylbewerberzahlen im Sommer 2015 schlug die Bundesagentur für Arbeit vor, einen rechtlichen Spurwechsel für Geflüchtete, deren Aufenthalt in Deutschland normalerweise in den Asylbestimmungen geregelt ist, zur „Blue Card“ und damit in die Arbeitsmigration zu ermöglichen, sodass hochqualifizierte Geflüchtete oder mögliche Auszubildende nicht in ihr Heimatland zurückkehren müssten um einen legitimen Antrag stellen zu können (Deutsche Welle 2015; Grote and Vollmer 2016). Allerdings wurde dieser Vorschlag vom Bundesinnenministerium abgelehnt, da dies als möglicher Anreiz zur Ausnutzung des Asylkanals und einer Erhöhung der Asylbewerberzahlen gedeutet wurde (Zeit Online 2015).

Nachdem Angela Merkel für ihre Entscheidung, Ende August 2015 die deutschen Grenzen für in Ungarn gestrandete Geflüchtete nicht zu schließen, kritisiert wurde, in Wählerumfragen potentielle Stimmen verlor und

14 Dabei sollten potentielle Zuziehende für ihre Ausbildung, Arbeitserfahrung und Sprachkenntnisse Punkte erhalten und ab einem gewissen Schwellenwert, der nach den Bedarfen des deutschen Arbeitsmarktes bemessen werde, nach Deutschland zuziehen dürfen (Diekmann 2015).

15 Siehe hierzu den Beitrag von Nils Imgarten in diesem Band. 
Unmut innerhalb der Bevölkerung mit der als ,nicht verkraftbar“ wahrgenommenen Flüchtlingssituation stieg (Infratest dimap 2015; SPON 2016), veränderte sich das Framing des Einwanderungsgesetzes der SPD. Diese warb für das Image eines Deutschlands, welches arbeitsmarktorientierte „Einwanderung , in kontrollierter Form““ (Steffen 2016) erlaube. Währenddessen betonte CDU-Generalsekretär Tauber, dass zunächst „Probleme mit den Flüchtlingen bewältigt sein [müssten], etwa die Rückführung und die Abschiebung abgelehnter Asylbewerber“ (Steffen 2016), also Personen die sich ,illegal' auf deutschem Territorium aufhalten, bevor man sich Fragen der Arbeitsmigration widmen könne. Stattdessen entschied sich die CDU auf ihrem Bundesparteitag im Dezember 2016 dazu, sich für die Wiedereinführung der Optionspflicht für Personen mit doppelter Staatsangehörigkeit und dafür, dass sich „,eine Situation wie die des Spätsommers 2015 (...) sich nicht wiederholen“ könne, solle und dürfe (Zeit Online 2016; EMN 2017, 21).

Im Bundestagswahlkampf 2017 proklamierte die SPD ihre Idee eines Einwanderungsgesetzes schließlich als eines, welches ,transparent und verständlich regelt, wer aus wirtschaftlichen Gründen nach Deutschland einwandern kann und wer nicht" (Hanewinkel 2017) und setzte sich gleichzeitig für eine Abwehr ,illegaler Migration“ bspw. mittels eines besseren Schutzes der europäischen Außengrenzen, ein. Die Unionsparteien stellten „das Gros ihrer migrationspolitischen Ideen unter die Überschrift ,Sicherheit im Inneren und nach außen“" und versprachen, Schleusersysteme zu bekämpfen und „Migrantinnen und Migranten ohne Schutzanspruch von der Überfahrt nach Europa“ (ebd.) abzuhalten. Im Koalitionsvertrag vom Januar 2018 wurden diese Position in Form des Punktes ,faire Mobilität fördern, jedoch missbräuchliche Zuwanderung in die Systeme der sozialen Sicherheit unterbinden" möchte (CDU, CSU, und SPD 2018, 7) ausgedrückt. Die dazugehörige Dichotomie in ,gewünschte“ und ,ungewünschte" Migrationsformen führt dabei nicht nur das Prinzip des Ausschlusses unerwünschter Mobilitätsformen aus dem vorangegangenen Koalitionsvertrag aus dem Jahre 2013, als es darum ging, anlässlich der Öffnung des Binnenmarktes für Bulgaren und Rumänen „Armutswanderungen innerhalb der EU“ und die ,ungerechtfertigten Inanspruchnahme[n] von Sozialleistungen durch EU-Bürger" entgegenzuwirken (CDU, CSU, und SPD 2013, 108), sondern vor allem die Praktiken der vorherigen Dekaden fort. Ebenso warnten kritische Stimmen während der Aushandlungsprozesse regelmäßig 
vor einer ,Zuwanderung in die Sozialsysteme“ (siehe z.B. Issig 2015; Bade 2017, 84; Welt 2018), diskutieren Risiken der ,Ausnutzung von Asyl“ (siehe z.B. Meurer 2015; Büscher and Lutz 2017; Steffen 2016) und fokussierten sich, wohl auch deshalb, auf den Zuzug von Fachkräften - obgleich gem. der 2018 Einigung nun ohne Vorrangprüfung durch das Arbeitsamt.

\section{FAZIT UND AUSBLICK: ZUR GESTALTUNG EINES NORMENWANDELS}

Dieser Beitrag diskutierte migrationspolitische Diskurse um Arbeitsmigration, Flucht und un-/erwünschte Mobilität in Deutschland mit einem soziohistorischen Blick auf die Jahre 1949-2005, um Debatten aus den Jahren 2014-18 um die mögliche Einführung oder Reformierung eines ,Einwanderungsgesetzes' bzw. eines ,Fachkräftezuwanderungsgesetzes‘ zu begreifen. Es zeigte sich zum einen, dass negative Wahrnehmungen, die während der frühen 1970er Jahre mit der Anwesenheit von Gastarbeitern und ihren Familienangehörigen, ab den späten 1970er Jahren mit Geflüchteten und teilweise Ausländern per se als gleichzeitige Konkurrenten und Bürden für den Wohlfahrtsstaat im Sinne von Arbeitsmarkt und Sozialsystemen verbunden wurden ausschlaggebend für den Verlauf der deutschen Migrationsdiskurse und -gesetzgebung waren. Aus einer Diskussion und Analyse von migrationspolitischer Sekundärliteratur, insbesondere des Werkes von Klaus J. Bade, sowie Primärquellen aus den relevanten Bundestagsdebatten, staatlichen Berichten und Statistiken ging hervor, dass der diskursive Fokus von ,Ausländerpolitik‘ seit den 1970er Jahren auf der Beschränkung und Regulierung von Zugängen zum deutschen Wohlfahrtsstaat, in Form des Arbeitsmarkts und den sozialen Sicherungssystemen, lag.

Anstatt sich proaktiv, vorrausschauend oder an den integrativen Bedarfen einer de facto seit Beginn der Gastarbeiterprogramme 1955 multikulturellen Gesellschaft und ihren Individuen zu orientieren, folgten die legalen Zuwanderungsmöglichkeiten in Deutschland primär den vorangegangenen rechtspositivistischen Strukturen (z.B. Asyl aus politischen und humanitären nicht etwa ökonomischen Gründen, Arbeitsmigration aus Gründen konkreter Nachfragen) und Reaktionismus auf gesellschaftliche Unzufriedenheiten (z.B. Anwerbestopp 1973, Reformierung des Ausländergesetzes 1990 und Asylkompromiss 1992-3). Anhand einer Analyse der Häufung 
und semantischen Wandlung der Figur des ,Wirtschaftsflüchtlings ' in den deutschen Migrationsdiskursen zeigte sich exemplarisch, wie ab den 1970er Jahren ein begriffliches Amalgam aus Flucht, Zuwanderung und Einwanderung entlang wirtschaftlicher Aspekte politisiert und zunehmend durch staatliche Regulierung hinsichtlich einer ,Abwehr' unliebsamer Eigenschaften versicherheitlicht wurde. Diese unliebsamen Eigenschaften umfassten sowohl die vermeintliche Wegnahme von Arbeitsplätzen als auch die Inanspruchnahme von Sozialleistungen im Falle von ausländischer Arbeitslosigkeit und wurden mit der Annahme verbunden, dass der Asylparagraph als Zugriffskanal zum deutschen Wohlfahrtsstaat anstelle eines Schutzes vor Verfolgung oder für Leib und Leben genutzt werde.

Es zeigte sich, dass Politiker nicht nur Erwartungen seitens der Bevölkerung, wirtschaftlicher Kräfte und Bedarfen der Globalisierung im Sinne eines ,liberalen Paradoxes“ (Hollifield 2011, 220; 2015, 188) ausgesetzt sind, sondern teilweise selber auch Fremdenfeindlichkeit und -skepsis im Kontext hervorriefen. Beispielsweise wurden Zuwanderungs- nicht gegen Abwanderungszahlen zu einem Saldo aufgerechnet, gar sogar in der ,Wanderungsstatistik" des statistischen Bundesamtes großzügig vermischt, Falschäußerungen getätigt die z.B. Gesamtschutzquoten zu niedrig und somit „Asylmissbrauchsquoten“ zu hoch ansetzen (z.B. Schily 1999) oder gar parteilich konzipiert (z.B. CDU/CSU 1991). Letztendlich erscheint also die Tatsache, dass Vorschläge der Bundestagsparteien aus den Jahren 20152018 die Un-/Erwünschtheit der Zuwanderung einer ausländischen Person anhand arbeitsmarktpolitischer Rationalitäten, sei es mittels eines Punktesystems oder konkreten Nachfragen von Arbeitgebern, festmachten nicht überraschend sondern eher sozialkonstruktivistisch stringent.

Daraus folgt, dass wenn deutsche Migrationspolitik einen Kurswechsel betreiben möchte, der ganzheitlich alle Zuwanderungsformen und ihre altruistischen wie egoistischen Motivationsfaktoren mitdenken möchte, zunächst Gründe für eine persistente negative Wahrnehmung von Ausländern und Migration innerhalb der deutschen Gesellschaft angegangen werden müssen (vgl. Bade 2015, 10; Claus Leggewie 1994, 215; Bade 2017b). Damit meine ich jedoch nicht, dass vor allem den Argumenten einer aufbrausenden Minderheit Genüge getan werden sollte (vgl. Zimmermann 2007, 180), sondern eher dass den darunter liegenden Ängsten vor Zuwanderung und unkontrollierten Zugängen zum Wohlfahrtsstaat untersucht und aktiv aufgearbeitet werden sollten. Beispielsweise ergab eine IOM Studie 
im Jahre 2015, dass 24 Prozent der reichsten und 47 Prozent der Ärmsten Deutschen Immigrationslevel gerne sinkend sowie 54 Prozent (reich) und 36 Prozent (arm) dieses gerne konstant sehen würden (IOM 2015, 46). Dies reflektiert sich auch im weltweiten Vergleich: Je besser bzw. exzellenter die ökonomischen Zustände in einem Land wahrgenommen werden, desto positiver sei auch die Wahrnehmung von ,Immigration“ - und umgekehrt (ebd., 17). Kurzum: Die Immigrationspolitik Deutschlands ist endogen zu seinem ökonomischen und sozialen System (Bodvarsson und Van den Berg 2013, 442), sodass die Erfolgsaussichten eines mobilitätspolitischen Wandels vor allem an die Zufriedenheit der aufnehmenden Bevölkerung mit ihrer eigenen sozioökonomischen Situation gekoppelt sind.

\section{LITERATUR}

Angenendt, Steffen, David Kipp, and Amrei Meier. 2017. Gemischte Wanderungen: Herausforderungen und Optionen einer Dauerbaustelle der Deutschen und Europäischen Asyl- und Migrationspolitik.“ https://www.bertelsmann-stiftung.de/fileadmin/files/Projekte/Migration _fair_gestalten/IB_Studie_Gemischte_Wanderungen_2017.pdf.

Bade, Klaus J. 2015. „,Wirtschaftsflüchtlinge“ und ,Scheinasylanten“: Zur Karriere Abschätziger Begriffe in Der deutschen Asyldiskussion." Aus Politik Und Zeitgeschichte (APUZ) 5.

Bade, Klaus J. 2017a. Migration, Flucht, Integration. Karlsruhe: Loeper Literaturverlag.

Bade, Klaus J. 2017b. „Migrationsforscher und Berater Bade zieht Bilanz: Achtung, Ausländer!“ Tagesspiegel.De, 25. April. http://www.tages spiegel.de/meinung/migrationsforscher-und-berater-bade-zieht-bilanzachtung-auslaender/19711722.html.

Bade, Klaus J. 2017c. „Gastbeitrag von Klaus J. Bade - Die Schattenseiten unserer Gier." Hannoversche Allgemeine Zeitung, 30. Juni. http:// www.haz.de/Sonntag/Gastkommentar/Die-Schattenseiten-unserer-Gier.

BAMF. 2005. „Migrationsbericht Des Bundesamtes für Migration und Flüchtlinge im Auftrag der Bundesregierung." Nürnberg. https://www.bamf.de/SharedDocs/Anlagen/DE/Publikationen/Migration sberichte/migrationsbericht-2005.pdf?_blob=publicationFile. 
BAMF. 2016. „Migrationsbericht 2015 - Zentrale Ergebnisse.“ Nürnberg. http:/www.bamf.de/SharedDocs/Anlagen/DE/Publikationen/Migrations berichte/migrationsbericht-2015-zentraleergebnisse.pdf?_blob=publi cationFile.

BAMF. 2017. „Das Bundesamt in Zahlen 2017: Asyl.“ Nürnberg. https:/www.bamf.de/SharedDocs/Anlagen/DE/Publikationen/Broschue ren/bundesamt-in-zahlen-2017-asyl.pdf?_blob=publicationFile.

Bauman, Zygmunt. 1998. Globalization: The Human Consequences. Columbia University Press.

Beauftragte der Bundesregierung für Migration. 2017. „Einwanderungsland Deutschland."https://www.bundesregierung.de/Content/Infomaterial/BP A/IB/EinwanderungslandDeutschland.pdf?_blob=publicationFile \&v= 4.

Beeger, Britta, and Kerstin Schwenn. 2018. „Union und SPD planen Einwanderungsgesetz." Frankfurter Allgemeine Zeitung, 9. Januar. http://www.faz.net/aktuell/wirtschaft/union-und-spd-einigen-sich-offen bar-auf-einwanderungsgesetz-15382575.html.

Bodvarsson, Örn Bodvar, und Hendrik Van den Berg. 2013. The Economics of Immigration: Theory and Policy. Springer.

Bröse, Johanna. 2018. Migration Und Arbeitsmarkt. Editiert von Johanna Bröse, Stefan Faas, Barbara Stauber, und Hrsg Flucht. Flucht: Herausforderungen Für Soziale Arbeit. Springer VS.

Bundesregierung. 2015. „Sommerpressekonferenz von Bundeskanzlerin Merkel,“ 31.August: https:/www.bundesregierung.de/Content/DE/Mit schrift/Pressekonferenzen/2015/08/2015-08-31-pk-merkel.html.

Büscher, Wolfgang, und Martin Lutz. 2017. „Ex-Innenminister Otto Schily: 'Das Asylrecht ist zu Einem Einwanderungsgesetz Mutiert.'“ Welt Online, 12. Juni. https://www.welt.de/politik/plus165409927/DasAsylrecht-ist-zu-einem-Einwanderungsgesetz-mutiert.html.

Butterwegge, Carolin. 2005. „Von Der Gastarbeiter-Anwerbung Zum Zuwanderungsgesetz.“ Bundeszentrale Für Politische Bildung. https:// www.bpb.de/gesellschaft/migration/dossier-migration/56377/migrations politik-in-der-brd? $\mathrm{p}=$ all.

Buzan, Barry. 1993. „Societal Security, State Security and Internationalisation." In Identity, Migration and the New Security Agenda in Europe, editiert von Ole Waever, 41-58. London: Pinter Publishers Ltd.\# 
BVerfG. 2012. Urteil des Ersten Senats vom 18. Juli 2012, 1 BvL 10/10; (BVerfGE 132, S. 134 - 179).

CDU/CSU. 2002. „Gemeinsames Wahlprogramm der Christlich Demokratischen Union Deutschlands (CDU) und der Christlich Sozialen Union (CSU) für die Bundestagswahl 2002." Documentarchiv. http:// www.documentarchiv.de/brd/2002/wahlprogramm_cdu_2002.html\#6.

CDU, CSU, und SPD. 2013. Deutschlands Zukunft gestalten: Koalitionsvertrag zwischen CDU, CSU und SPD: 18. Legislaturperiode, 2013: https://www.bundesregierung.de/Content/DE/_Anlagen/2013/201 3-12-17koalitionsvertrag.pdf;jsessionid=3FFA0F55833990528376A1 A442B39FD2.s2t1? blob=publicationFile\&v=3.

CDU, CSU, und SPD. 2018. Ein neuer Aufbruch für Europa, Eine neue Dynamik für Deutschland, ein neuer Zusammenhalt für unser Land: Koalitionsvertrag zwischen CDU, CSU und SPD: 19. Legislaturperiode, 2018.https://www.bundesregierung.de/Content/DE/_Anlagen/2018/03/2 018-03-14-koalitionsvertrag.pdf?_blob=publicationFile \&v=5.

Chaloff, Jonathan, and Georges Lemaitre. 2009. „Managing Highly-Skilled Labour Migration: A Comparative Analysis of Migration Policies and Challenges in OECD Countries.“ 79. Social, Employment and Migration. Paris.

Claus Leggewie. 1994. „Das Ende Der Lebenslügen: Plädoyer Für Eine Neue Einwanderungspolitik.“ In Das Manifest Der 60, editiert von Klaus J. Bade, 55-60. München: C.H. Beck.

Constant, A. F., and B. N. Tien. 2011. „Germany's Immigration Policy and Labor Shortages." 41. IZA Research Report.

Deutsche Welle. 2015. „German Job Agency Calls to Expand 'Blue Card' to Academic Refugees." Deutsche Welle, 19. Juli. http://www.dw.com/ en/german-job-agency-calls-to-expand-blue-card-to-academic-refugees/ a-18594799.

Deutscher Bundestag. 1992. „Plenarprotokoll 12/89.“ http://dipbt.bundes tag.de/doc/btp/12/12089.pdf.

Deutscher Bundestag. 1993. „Plenarprotokoll 12/160،“ http://dipbt.bundes tag.de/doc/btp/12/12160.pdf.

Deutscher Bundestag. 2000. „Sechster Familienbericht Familien ausländischer Herkunft in Deutschland Leistungen - Belastungen - Herausforderungen und Stellungnahme der Bundesregierung." http://dipbt. bundestag.de/doc/btd/14/043/1404357.pdf. 
Deutscher Bundestag. 2015. „Plenarprotokoll 18/85““ http://dipbt.bundes tag.de/doc/btp/18/18085.pdf.

Diekmann, Florian. 2015. „Flüchtlinge: Was Asyl mit einem Einwanderungsgesetz zu tun hat." Spiegel Online, 26. Juli. http://www. spiegel.de/politik/deutschland/fluechtlinge-was-asyl-mit-einem-einwan derungsgesetz-zu-tun-hat-a-1045399.html.

Eichenhofer, Johannes, and Carsten Hörich. 2018. „Vom Zuwanderungszum Einwanderungsgesetz?" In Hohenheimer Horizonte: Festschrift für Klaus Barwig, editiert von Stephan Beichel-Benedetti and Constanze Janda, 1st ed., 428-41. Baden-Baden: Nomos Verlagsgesellschaft mbH \& Co. KG.

EMN. 2017. „Migration, Integration, Asyl: Politische Entwicklungen in Deutschland 2016." Jährlicher Bericht der Deutschen Nationalen Kontaktstelle für das Europäische Migrationsnetzwerk. Nürnberg. http: //www.bamf.de/SharedDocs/Anlagen/DE/Publikationen/EMN/Politikbe richte/emn-politikbericht-2016-germany.pdf?_blob=publicationFile.

FAZ. 2015. „Thomas de Maizière gegen Einwanderungsgesetz Und Punktesystem.“" Frankfurter Allgemeine Zeitung. http:/www.faz.net/ aktuell/politik/thomas-de-maiziere-gegen-einwanderungsgesetz-und-pu nktesystem-13411317.html.

Foroutan, Naika. 2018. „Ambivalent Germany. How to Deal with Migration, Muslims and Democracy." In Fortress Europe? Challenges and Failures of Migration and Asylum Policies, editiert von Annette Jünemann, Nicolas Fromm, und Nikolas Scherer, 123-38. Springer VS.

Gauck, Joachim. 2014. „Einbürgerungsfeier Anlässlich 65 Jahre Grundgesetz.“ Der Bundespräsident, May 22. http://www.bundes praesident.de/SharedDocs/Reden/DE/Joachim-Gauck/Reden/2014/05/ 140522-Einbuergerung-Integration.html.

Grote, Janne, und Michael Vollmer. 2016. „Wechsel Zwischen Aufenthaltstiteln und Aufenthaltszwecken in Deutschland.“ 67. Working Paper. Nürberg. https:/ec.europa.eu/home-affairs/sites/homeaffairs/ files/what-we-do/networks/european_migration_network/reports/docs/ emn-studies/wp_67_wechsel_zwischen_aufenthaltstiteln_deutsch.pdf.

Hampshire, James. 2011. „Disembedding Liberalism? Immigration Politics and Security in Britain since 9/11." In Immigration Policy and Security: U.S., European and Commonwealth Perspectives, editiert von T.E. Givens et al., 109-29. London: Routledge. 
Hanewinkel, Vera. 2017. „Wie stehen die deutschen Parteien zu den Themen Migration, Integration, Flucht Und Asyl?“ Bundeszentrale Für Politische Bildung. http://www.bpb.de/gesellschaft/migration/laender profile/255670/parteien-zu-migration-integration-flucht-und-asyl\#foot node1-1.

Hollifield, James F. 2011. „Migration and the Global Mobility of Labor: A Public Goods Approach." In Global Mobility Regimes, editiert von Rey Koslowski, 219-40. New York [u.a.]: Palgrave Macmillan.

Hollifield, James F. 2015. „The Politics of International Migration: How Can We 'Bring the State Back In?'“ In Migration Theory: Talking across Disciplines, editiert von Caroline Brettell and James Hollifield, 3. ed., 183-238. New York, NY [u.a.]: Routledge.

Infratest dimap. 2015. „Merkels Flüchtlingspolitik bleibt umstritten, Größte Zustimmung bei Grünen-Anhängern.“ ARD-Morgenmagazin. https:// www.infratest-dimap.de/umfragen-analysen/bundesweit/umfragen/aktu ell/merkels-fluechtlingspolitik-bleibt-umstritten-groesste-zustimmungbei-gruenen-anhaengern/.

Infratest dimap. 2017. „ARD-DeutschlandTREND September 2017 II / KW37.“ Bedeutung von Themen Für Die Wahlentscheidung II. https://www.infratest-dimap.de/umfragen-analysen/bundesweit/arddeutschlandtrend/2017/september-ii/.

IOM. 2015. „How the World Views Migration.“ Geneva. https:// publications.iom.int/system/files/how_the_world_gallup.pdf.

Infratest dimap. 2016a. „Global Migration Trends Factsheet.“ International Organization for Migration. http://gmdac.iom.int/global-migrationtrends-factsheet.

Infratest dimap. 2016b. „Summit on Refugees and Migrants Begins as IOM Joins the United Nations | International Organization for Migration." International Organization on Migration, September 18. https://www.iom.int/news/summit-refugees-and-migrants-begins-iomjoins-united-nations.

Issig, Peter. 2015. „Horst Seehofer und der Spruch vom Weltsozialamt'.“ Welt Online, 19. Februar. https://www.welt.de/politik/deutschland/ article137642111/Horst-Seehofer-und-der-Spruch-vom-Weltsozialamt. html.

Klaus J. Bade, ed. 1994. „Das Manifest der 60: Deutschland und die Einwanderung.“ München: C.H. Beck. 
Leggewie, Claus. 2000. „Integration and Segregation.“ In Migrationsreport 2000: Fakten, Analysen, Perspektiven, editiert von Klaus Münz and Rainer Münz, 85-109. Frankfurt, New York: Campus.

Mehrländer, Ulrike. 1987. „Sociological Aspects of Migration Policy: The Case of the Federal Republic of Germany.“ International Migration 25 (1): 87-96.

Meurer, Friedbert. 2015. „CDU-Politiker Spahn plädiert weiter für Einwanderungsgesetz.“ Deutschlandfunk, 15. Januar. http://www.deutsch landfunk.de/einwanderungsdebatte-cdu-politiker-spahn-plaediert-weit er.694.de.html?dram:article_id=308715.

Müller, Andreas. 2012. „Migrationskontrolle ohne Grenzkontrolle. Deutschland in der erweiterten EU." Gesellschaft, Wirtschaft, Politik 1: 914.

Müller, Doreen. 2010. Flucht und Asyl in Europäischen Migrationsregimen: Metamorphosen einer umkämpften Kategorie am Beispiel der EU, Deutschlands und Polens. Göttingen: Universitätsverlag Göttingen.

O’Nions, Helen. 2014. Asylum - A Right Denied. A Critical Analysis of European Asylum Policy. Farnham/Burlington: Ashgate.

Oberndörfer, Dieter. 1994. „Multikulturalismus in der Einwanderungsgesellschaft.“ In Das Manifest der 60: Deutschland und die Einwanderung, editiert von Klaus J. Bade, 34-37. München: C.H. Beck.

Oltmer, Jochen. 2014. „Einwanderung als Normalität - Eine Botschaft der Rede des Bundespräsidenten Joachim Gauck vom 22.05.2014.“ Zeitschrift für Ausländerrecht und Ausländerpolitik 34 (8): 259-60.

OECD. 2016. „States of Fragility 2016: Understanding Violence.“ Paris: Organization for Economic Development. http://www.oecdilibrary.org/docserver/download/4316101e.pdf?expires=1504977094\&i $\mathrm{d}=\mathrm{id} \&$ accname $=$ guest\&checksum $=9$ DE000BB431DB6D44103A520D0 91E5C6.

Reißlandt, Carolin. 2002. „Rot-Grüne Migrationspolitik und die Zuwanderungsdebatte. vom 'Paradigmenwechsel' zum Wahlkampfthema?" In Deutschland auf den Weg gebracht: Rot-Grüne Wirtschafts- und Sozialpolitik zwischen Anspruch und Wirklichkeit, editiert von Kai Eicker-Wolf u.a., 213-51. Marburg.

Roman Eckendorff, and Markus Stock. 2016. „Methodenbericht: Statistik über die Arbeitsgenehmigungen-EU und Zustimmungen.“ Nürnberg. http://statistik.arbeitsagentur.de. 
Schammann, Hannes. 2018. „Reassessing the Opinion-policy Gap. How PEGIDA and the AfD Relate to German Immigration Policies." In Fortress Europe? Challenges and Failures of Migration and Asylum Policies, editiert von Annette Jünemann, Nicolas Fromm, and Nikolas Scherer, 139-58. Springer VS.

Schneider, Jan. 2007. „Rückblick: Zuwanderungsgesetz 2005.“ Bundeszentrale für politische Bildung. http://www.bpb.de/gesellschaft/ migration/dossier-migration/56351/zuwanderungsgesetz-2005? $\mathrm{p}=$ all.

Simantke, Elisa. 2015. „Punktesystem für Migranten?: SPD und CDU Streiten über Einwanderungsgesetz - Politik - Tagesspiegel.“ Tagesspiegel, 3. März. https://www.tagesspiegel.de/politik/punktesystem-fuer -migranten-spd-und-cdu-streiten-ueber-einwanderungsgesetz/1145069 4.html.

SPON. 2015. „De Maizière fordert gezielte Werbung um Fachkräfte.“ Spiegel Online, 14. April. http://www.spiegel.de/politik/deutschland/ zuwanderung-thomas-de-maiziere-fordert-gezielte-werbung-a-1028577. html.

SPON. 2016. „Mehrheit der Deutschen ist gegen aktuelle Flüchtlingspolitik.“ Spiegel Online, 15. Februar. http://www.spiegel.de/politik/ deutschland/fluechtlinge-deutsche-wenden-sich-laut-umfrage-gegen-an gela-merkel-a-1072192.html.

Steffen, Tilman. 2016. „Migration nach Punkten.“ ZEIT Online, 7. November. https://www.zeit.de/politik/deutschland/2016-11/einwanderungs gesetz-spd-punktesystem-union.

Takle, Marianne. 2007. German Policy on Immigration - from Ethnos to Demos? Frankfurt am Main: Europäischer Verlag der Wissenschaften.

Thränhardt, Dietrich. 2009. „Deutschland 2008: Integrationskonsens, Pessimistische Erinnerungen und neue Herausforderungen durch die Globalisierung." In Einwanderungsgesellschaft Deutschland: Wege zu einer sozialen und gerechten Zukunft, editiert von Günther Schultze, Axel Schulte, Dietrich Thränhardt, Ursula Boos-Nünning, Harmut Häussermann, and Urmila Goel, 45-59. Berlin: Friedrich-EbertStiftung.

Tsoukala, Anastasia. 2011. „Turning Immigrants into Security Threat: A Multi-Faceted Process.“ In Security, Insecurity and Migration in Europe, editiert von G. Lazaridis, 179-200. Surrey: Ashgate. 
Uwe Hunger, and Sascha Krannich. 2017. „Einwanderung neu gestalten transparent, attraktiv, einfach.“ http://library.fes.de/pdf-files/wiso/133 74.pdf.

Welt. 2018. „Seehofer zu Familiennachzug: „Wir wollen keine Zuwanderung in die Sozialsysteme“." Welt Online, 9. April. https://www. welt.de/politik/deutschland/video175285540/Seehofer-zu-Familiennach zug-Wir-wollen-keine-Zuwanderung-in-die-Sozialsysteme.html.

Zeit Online. 2015. „Innenministerium lehnt Blue Card für Flüchtlinge ab.“ Zeit Online, 20. Juli. https:/www.zeit.de/politik/deutschland/ 2015-07/fluechtlinge-asylbewerber-bleiberecht-blue-card.

Zeit Online. 2016. „2016 Hat die Welt schwächer und Instabiler gemacht.“ Zeit Online, 6. Dezember. https://www.zeit.de/politik/deutschland/2016 -12/angela-merkel-cdu-parteitag-essen-wiederwahl-parteichefin-wahlka mpf-2017.pdf

Zimmermann, Klaus F. 2007. Immigration Policy and the Labor Market. Berlin, Heidelberg: Springer.

Zygmunt Bauman. 2000. Liquid Modernity. Cambridge: Polity Press. 

An den Grenzen des Rechts 



\section{Das Visum und die Verlagerung der Grenzkontrolle}

Thea Kirsch

While the speed and reach of human mobility have certainly increased over the past two centuries, this mobility is hardly >new<; what is relatively new is the criminalization of movement.

Noora A. Lori (2017: 747)

\section{1. „WARUM KOMMEN FLÜCHTLINGE EIGENTLICH NICHT MIT DEM FLUGZEUG? «}

Im 21. Jahrhundert sind Menschen, Informationen, Waren, Dienstleistungen und Kapital so mobil wie nie zuvor. Mit fortschreitender Digitalisierung vernetzt die Welt sich immer stärker - über staatliche Grenzen und virtuelle Räume hinweg. Doch während die internationale Unternehmensberatung McKinsey ein neues Zeitalter der Globalisierung ausrufen lässt, erzählt die »New Era of Global Flows« (McKinsey Global Institute 2016) eine Geschichte selektiver Im-/Mobilisierung. Zwar folgt die Personenmobilität dem Globalisierungstrend eines allgemeinen Anstiegs an grenzüberschreitenden Bewegungen, aber im internationalen Vergleich sind individuelle Chancen auf Mobilität stark ungleich verteilt (vgl. Laube 2013: 13-14). Nur wenige Privilegierte genießen den Schein einer Welt ohne Grenzen. Insbesondere Staatsbürger/innen des globalen Nordens profitieren von einem bedeutend höheren Maß an Bewegungsfreiheit (vgl. Mau et al. 2015: 1192). 
Sie sind eine mobile Elite, deren Pass es vermag Türen zu öffnen, die den ewig Lokalen verschlossen bleiben.

Unsere individuelle Chance auf Mobilität ist einerseits abhängig von den finanziellen, technischen und sozialen Ressourcen, auf die wir zugreifen können. Nach dem Prinzip der territorialen Souveränität ist es jedoch gleichzeitig das Recht eines jeden Staates zu entscheiden, wem der Zugang zum eigenen Staatsgebiet gewährt wird und wem nicht (vgl. Laube 2013: 14-15). An der Grenzlinie trennt die Entscheidung zwischen Grenzöffnung und Grenzschließung das Innere des Staates vom Äußeren und gibt der nationalstaatlichen Rechtsordnung ihren spezifischen physisch-materiellen Geltungsbereich. Wer sich außerhalb der territorialen Grenzen der eigenen Staatsangehörigkeit frei bewegen will, benötigt grundsätzlich ein Visum für den Grenzübertritt — oder verstößt ohne Einreise- und Aufenthaltserlaubnis gegen den vom Zielland gesetzten Rahmen der Legalität (vgl. Torpey 1998: 239-249). In einer Welt, die scheinbar immer enger zusammenrückt, verbleibt die territoriale Gliederung nationalstaatlicher Souveränität als potentiell weitreichende Einschränkung für die Bewegungsfreiheit. Gleichzeitig erleichtert ein Großteil der Staaten weltweit den Staatsbürger/innen ausgewählter anderer Länder den Zugang zum eigenen Staatsgebiet durch die Aufhebung der Visumpflicht. Eine solche generalisierte Erlaubnis für die grenzübergreifende Mobilität aller Angehörigen eines Staates behandelt Einreisewillige bestimmter nationaler Herkunft bevorzugt. Demgegenüber verlagert die Auferlegung der Visumpflicht die Grenzkontrolle vom Zielland an den Aufbruchsort jener Staatsbürger/innen, die sich für eine Einreise- und Aufenthaltserlaubnis erst in einem individuellen Antragsverfahren beweisen müssen (vgl. Laube 2013: 14-15).

Das Visum als Instrument der räumlich flexibilisierten Kontrolle zeigt beispielhaft, dass »Grenzen als Sortiermaschinen« (Mau 2011) sich ausdehnen und der Bewegung jener Individuen bis zu ihrem Ursprung folgen, deren Immobilisierung sie zum Ziel haben. Wer ohne offiziell anerkannte Einreise- und Aufenthaltspapiere aufbricht, wird ab dem Zeitpunkt des undokumentierten Aufbruchs mit dem Label der >Illegalität außerhalb des Rechts des Ziellandes gestellt. So entschlüsselt »die Frage nach der legalen Präsenz« (Schulze Wessel 2017: 177) eine neue Logik der Grenzkontrolle. Staatliche Visumpolitik trennt den Ort der Entscheidung zwischen Grenzöffnung und Grenzschließung räumlich von der territorialen Grenzlinie, an 
der die rechtliche Zuständigkeit des Ziellandes beginnt (vgl. Laube 2010: $1-2)$.

Die Ungleichbehandlung von Menschen basierend auf einer vereinheitlichenden Kategorisierung von nationaler Zugehörigkeit wirft dringende ethische und moralische Bedenken auf. Aus einer normativen Perspektive ist das Recht auf Bewegungsfreiheit grundlegende Voraussetzung für individuelle Entscheidungsautonomie. Grenzübergreifende Mobilität kann Schutz vor Gewalt, bessere Bildungschancen, steuerliche Vorteile oder einen Last-Minute-Trip am Wochenende bedeuten. Bewegungsfreiheit ist stets Ausgangspunkt für »das Interesse, sich selbst Ziele setzen zu können und sein Leben weitgehend selbstbestimmt zu gestalten« (Angeli 2011: 171). Je selektiver Staaten also grenzübergreifende Mobilität einschränken, desto stärker sind unsere Lebenschancen, Entscheidungsmöglichkeiten und Handlungsräume nahezu willkürlich abhängig vom Zugehörigkeitsprinzip der Staatsbürgerschaft durch Geburt.

Dieser Beitrag fragt bewusst provokativ: »Warum kommen Flüchtlinge eigentlich nicht mit dem Flugzeug?« und kritisiert damit gegenwärtige Migrations- und Grenzpolitiken liberaler Demokratien für die politische Entscheidung, wissentlich und willentlich die individuelle Chance auf Mobilität von Menschen bestimmter Herkunft zu beschränken. Vor dem Hintergrund der Frage globaler Gerechtigkeit soll im ersten Teil dieses Beitrags dargestellt werden, dass die weltweite Ungleichverteilung von Mobilitätsrechten als historisch gewachsenes System struktureller Diskriminierung begriffen werden muss. So macht die kritische Betrachtung des globalen Visumregimes deutlich, dass die Asymmetrie der Bewegungsfreiheit das Statusgefälle zwischen den Ländern des globalen Nordens und den Ländern des globalen Südens fortlaufend reproduziert und verschärft. Darüber hinaus ist es Ziel dieses Beitrags aufzuzeigen, dass die Visumpolitik der liberalen Demokratien des globalen Nordens insbesondere Länder benachteiligt, aus denen Menschen kommen, deren besonderes Schutzbedürfnis das internationale Menschen- und Völkerrecht anerkennt. Diese empirische Beobachtung ist im zweiten Teil dieses Beitrags entscheidend für die Analyse des europäischen Visumregimes und seine Folgen für den Zugang zu Asylverfahren in der Europäischen Union. Hier sind die räumlichen Dimensionen des europäischen Visumregimes Ausgangspunkt für die Schlussfolgerung, dass Strategien der Grenzverlagerung neue politische Handlungsspielräume schaffen, welche als Rechtfertigung für die selektive Zugangskon- 
trolle zum rechtlichen Geltungsbereich liberaler Normen dienen. Die Visumpolitik der Europäischen Union beschränkt für Menschen aus den Ländern des globalen Südens legale Wege nach Europa und legitimiert durch das räumliche Auseinanderfallen von Grenzkontrolle und Grenzlinie gleichzeitig, dass europäische Politik jenseits des Schengenraums gegen die Verpflichtung auf das internationale und europäische Menschen- und Flüchtlingsrecht verstößt. Auf diese Weise nimmt die Europäische Union nicht nur in Kauf, dass Menschen in Ermangelung legaler Einreise- und Aufenthaltsmöglichkeiten auf immer gefährlichere Routen ausweichen müssen, sondern kriminalisiert undokumentiert Wandernde als >Illegale

Dieser Beitrag ist ein Plädoyer dafür, den Blick für die europäische Visumpolitik als eine räumliche Strategie des systematischen Rechtsvorenthalts zu öffnen. Visumfreies Reisen schützt den Zugang zum Recht auf Asyl. Hingegen hat die selektive Einschränkung visumfreien Reisens für Menschen, die vor Krieg, politischer Verfolgung oder Armut fliehen, tödliche Folgen. Es ist ein direktes Ergebnis politischer Entscheidungen in der europäischen Migrations- und Grenzpolitik, dass es für jene Menschen kaum sichere und legale Wege nach Europa gibt, welche am stärksten angewiesen sind auf die Hilfe eines schützenden Europas - eines Europas einst gegründet auf »die Achtung der Menschenwürde, Freiheit, Demokratie, Gleichheit, Rechtsstaatlichkeit und die Wahrung der Menschenrechte einschließlich der Rechte der Personen, die Minderheiten angehören «. ${ }^{1}$

\section{DAS GLOBALE VISUMREGIME}

Historisch betrachtet wurde mit dem Visum eines der ersten staatlichen Instrumente etabliert, um die Grenzkontrolle vom Zielland an den Aufbruchsort zu verlagern. So ist die Auferlegung der Visumpflicht eine Strategie für die Politik der Kontrolle aus der Ferne; der »Remote Control (Zolberg 2006: 244). Die Kontrollwirkung von Visumpolitik wurde zusätzlich verschärft durch die Einführung sogenannter `Carrier Sanctions`, mit denen private Beförderungsunternehmen (insbesondere Fluggesellschaften)

1 Siehe AEUV in der Version von Lissabon, Artikel 2. 
unter erheblichen, »abschreckend[en] $\aleph^{2}$ Sanktionen dazu verpflichtet werden nur Kunden mit gültigen Einreise- und Aufenthaltstiteln zu befördern (vgl. Rodenhäuser 2014: 223-226). Für diese räumliche Flexibilisierung der Grenzkontrolle haben Didier Bigo und Elspeth Guild den treffenden Begriff des »Policing at a Distance« (Bigo/Guild 2005) geprägt.

Visumkriterien und Vergabeverfahren verschieben den Aushandlungsprozess für die individuelle Einreiseerlaubnis vor die eigentlichen Grenzen des Ziellandes. Hingegen erlebten Reisende die territoriale Grenzlinie in der Zeit vor der umfassenden Standardisierung des Pass- und Visumsystems als einzigen Bezugspunkt der Zugangskontrolle, räumlich gebunden an das Staatsgebiet. ${ }^{3}$ Bis zum Moment der Grenzkontrolle waren alle Reisenden gleich, erst mit der Entscheidung zwischen Grenzöffnung und Grenzschließung spürten sie die Selektivität der Grenze (vgl. Schulze Wessel 2016: 51-52). Heute wird in Konsulaten, Botschaften und anderen Behördenstellen bereits am Ort der Ausreise entschieden, wer mit einem Visum legal in Richtung des Ziellandes aufbrechen darf oder nicht. Während die Grenzlinie »nur noch ein Bezugspunkt für die Kontrolle von Mobilität« (Chojnacki/Paping 2016: 21; Hervorhebung im Original) ist, bleibt der souveräne Herrschaftsanspruch über den Zugang zum Staatsgebiet unverändert.

Die Verlagerung der Grenzkontrolle errichtet jedoch nicht gegenüber jeder Nation gleichermaßen zusätzliche Mobilitätsbarrieren. Global betrachtet hat eine kontinuierliche Ausweitung von Mobilitätsrechten durch eine steigende Anzahl von Abkommen über Visumfreiheit stattgefunden.

2 Siehe Amtsblatt der Europäischen Gemeinschaft, OJ L 187 vom 10.7.2001, Artikel 4, S. 46.

3 Mit Ausbruch des Ersten Weltkriegs wurden Pass und Visum zur weltweiten Norm. Bereits im späten 19. Jahrhundert begann jedoch die schrittweise Herausbildung eines internationalen Passsystems, das grenzübergreifende Mobilität in Verbindung mit zusätzlichen Reiseautorisierungen wie dem Geleitbrief oder später dem Visum regulierte (vgl. Mau/Brabandt 2011: 4). Seit den frühen Anfängen staatlicher Mobilitätskontrolle sind manche Individuen aufgrund von Klassen- oder Rassenzuschreibungen das Ziel selektiver Im-/Mobilisierung gewesen, so zum Beispiel chinesische Arbeiter/innen unter dem US-amerikanischen `Chinese Exclusion Act`von 1882 (vgl. Mau et al. 2012: 20). 
Jedoch haben vor allem die Regionen des globalen Nordens von der vermehrten Aufhebung der Visumpflicht profitiert. Staatsbürger/innen der meisten Länder in Europa, Nordamerika und Südamerika erleben nationale Grenzen immer weniger als Hindernis. Hingegen sind die Länder in den Regionen des globalen Südens von dieser Entwicklung überwiegend ausgeschlossen (vgl. Mau et al. 2015: 1192-1196). Im Zeitraum von 1969 bis 2010 hat die Region Asien-Pazifik keinen wesentlichen Zuwachs an Mobilitätsrechten erfahren. Die Länder des afrikanischen Kontinents haben im Durchschnitt sogar verstärkt die Visumpflicht auferlegt bekommen und somit an Bewegungsfreiheit verloren (vgl. ebd. 1203-1206). Im Ländervergleich des globalen Nordens stimmen die Nationen mit der Erlaubnis für visumfreies Reisen immer stärker überein. Während die Staatsbürger/innen der Länder des globalen Nordens in eine Mehrzahl der Länder weltweit für bis zu 90 Tage visumfrei einreisen können, bleibt dieses Privileg den Ländern des globalen Südens umgekehrt verwehrt: »Mobilitätserleichterungen werden zu Clubgütern der westlichen Welt« (Mau 2011: 79). Die weltweite Verteilung von Mobilitätsrechten ist daher stark polarisiert.

Seit zwölf Jahren veröffentlichen Henley \& Partners mit dem >Visa Restrictions Index mungen in Zusammenarbeit mit der International Air Transport Association, dem internationalen Dachverband des Lufttransportgewerbes und Betreiber der größten Datenbank von Reiseinformationen. Nach diesem Index von Henley \& Partners (Stand 2017) sind die Nationen mit dem größten Anteil an Mobilitätsrechten: Deutschland (Platz 1), Schweden (Platz 2) sowie Dänemark, Finnland, Italien, Spanien und die USA (Platz 3). Henley \& Partners aktualisieren das Ranking jedes Jahr, indem sie überprüfen, in wie viele Länder die Staatsbürger/innen einer Nation visumfrei einreisen dürfen. Deutschland steht das zweite Jahr infolge an der Spitze des Mobilitätsrankings mit der Möglichkeit zur visumfreien Einreise in 176 Ländern von möglichen 218 Ländern. ${ }^{4}$ Im Gegensatz dazu sind Syrien (Platz 101),

4 Das Ranking bewertet die Mobilitätsrechte der Pässe von 199 Nationen, darunter die 193 Mitgliedstaaten der Vereinten Nationen, der Vatikan, die Palästinensischen Autonomiegebiete, Kosovo, Taiwan, Hong Kong (SAR China) und Macao (SAR China). Henley \& Partners zählen 219 Territorien als mögliche 
Pakistan (Platz 102), Irak (Platz 103) und Afghanistan (Platz 104) die Länder, deren Staatsbürger/innen den geringsten Umfang an Mobilitätsrechten besitzen. Staatsbürger/innen dieser Länder stehen weniger als 30 visumfreie Ausreisemöglichkeiten offen (vgl. Henley\&Partners 2017).

Im Jahr 2017 befinden sich alle 35 OECD-Staaten unter den 64 Ländern, die das obere Drittel der Rankingplätze 1-35 des Index belegen. Auffällig ist bei Betrachtung der Gruppe der mobilen bis sehr mobilen Nationalitäten (Ranking 1-35), dass Nicht-OECD-Staaten sich entweder durch ein verhältnismäßig hohes BIP (z.B. Singapur, Hong Kong und Brunei Darussalam) auszeichnen oder durch ihre Mitgliedschaft in einer supranationalen Organisation regionaler Integration (z.B. Kroatien, Bulgarien, Litauen, Lettland, Rumänien, Zypern und Malta). ${ }^{5}$ Mit Ausnahme von Haiti und der Dominikanischen Republik liegen die verbleibenden 78 Länder des unteren Drittels der Rankingplätze 69-104 in Afrika und Asien (vgl. ebd.). Gerade in den Ländern an der Peripherie des globalisierten Kapitalismus sind jedoch immer mehr Menschen gezwungen aufgrund von Krieg, politischer Verfolgung und/oder Armut ihre Heimat zu verlassen (vgl. Hess et al. 2015: 2). Die Gegenüberstellung der wenig mobilen bis immobilen Länder (Plätze 69-104) zeigt, dass der Großteil der Staaten unter großer Armut und Ressourcenknappheit leidet (z.B. Yemen, Eritrea, Äthiopien und Haiti), die eigene Bevölkerung nicht vor den Folgen von Krieg, Verfolgung und Vertreibung schützt (z.B. Afghanistan, Irak, Pakistan und Syrien), eine starke

Reiseziele. Daraus ergibt sich ein Höchstwert von 218 visumfreien Einreisemöglichkeiten als Bewertungsmaßstab, der dem >Visa Restrictions Index`zugrunde liegt. Es werden innerhalb des Rankings keine Punkt vergeben für die Möglichkeit, dass Staatsbürger/innen visumfrei in ihr Heimatland einreisen können (vgl. Henley \& Partners 2017).

5 Alle Mitgliedstaaten der Europäischen Union befinden sich im Jahr 2017 unter den ersten 25 Rankingplätzen des >Visa Restrictions Index . Auf den Plätzen 20 und 21 bilden Bulgarien, Kroatien und Rumänien das Schlusslicht der EUMitgliedstaaten (vgl. Henley\&Partners 2017). Seit ihrem Beitritt zur Europäischen Union haben sich die drei Länder im Durchschnitt um etwa 11 Listenplätze verbessert. Im internationalen Vergleich garantiert die Personenfreizügigkeit innerhalb des Schengenraums für Unionsbürger/innen ein besonders hohes Maß an Mobilität. 
Zuwanderung von Flüchtenden erfährt (z.B. Libyen, Libanon und Jordanien) und/oder mehrheitlich muslimisch ist (z.B. Iran, Algerien, Tunesien, Azerbaijan und Saudi Arabien) (vgl. Henley\&Partners 2017). Die Visumpolitik der Länder des globalen Nordens kommt so einem rechtlichen Schutzwall gleich, der sich nicht in einem allgemeinen Abschottungsdoktrin erschöpft, sondern nur Staatsbürger/innen des globalen Südens entlang wirtschaftlicher, politischer und kultureller Stigma vom Privileg grenzübergreifender Mobilität ausschließt.

Henley \& Partners sind führende Berater für >Citizenship-byInvestment/-Programme; dem Geschäft der Staatsbürgerschaft per Investition. Das Unternehmen unterstützt nach eigener Aussage wohlhabende Individuen und Familien, eine zweite Staatsbürgerschaft im Rahmen staatlicher Investitionsprogramme zu erwerben - und die damit einhergehenden Mobilitätsrechte zur visumfreien Einreise in andere Länder (vgl. Henley\&Partners 2018). Das Western Hemisphere Department des Internationalen Währungsfonds stuft `Citizenship-by-Investment`-Programme als expandierende Branche ein, welche die Mobilitätsbedürfnisse einer kleinen aber schnell wachsenden Gruppe von sehr vermögenden Privatpersonen bedient (vgl. Gold/El-Ashram 2015: 48). Für die millionenschwere Kundschaft von Vermittlungsagenturen wie Henley \& Partners ist eine umfangreiche Investition im Tausch für einen zweiten Pass die einfachste Lösung, um mögliche Einschränkungen durch die eigene Staatsbürgerschaft zu überwinden:

»Ein New Yorker Unternehmer, der schnell zu einem Geschäftspartner nach Paris reisen muss, um einen Vertrag abzuschließen, braucht dafür nicht mehr als zwölf Stunden einzuplanen - und ist damit im Vorteil gegenüber seinem Konkurrenten aus Botswana, der einen Visumsantrag stellen, Bearbeitungsgebühren bezahlen und sich mehrere Tage gedulden muss, bis er - wenn er Glück hat - den kostbaren Sesamöffne-dich erhält« (Bréville 14.02.2014).

Der wachsende Markt für den Investitionshandel mit der Staatsbürgerschaft verdeutlicht, dass individuelle Bewegungsfreiheit ein stark selektiv reguliertes Gut ist von hohem immateriellen und materiellen Wert. Wer genug Geld besitzt, kann sich durch eine zweite Staatsbürgerschaft im Tausch gegen einen Millionenbetrag jene Mobilität dauerhaft sichern, die andere erst für jeden visumpflichtigen Ausreisewunsch beantragen und bezahlen 
müssen. Das Recht des Geldes schafft so einen Ausweg aus den Grenzen der Nation, die durch die Verbindung von Geburt und Staatsangehörigkeit ein Willkürsystem ungleicher Privilegien aufrechterhalten. Das Tauschgeschäft ist Ausdruck des globalen Machtgefälles, das es kapitalisiert. Hieraus müssen wir die Schlussfolgerung ziehen, dass Reichtum Menschen mobil macht: Entweder in Form des BIPs des Herkunftsland oder des eigenen Privatvermögens.

Am Beispiel der OECD-Staaten belegt der Henley \& Partners $>$ Visa Restrictions Index ihrer Eigenwahrnehmung als Gleiche unter Gleichen besondere Mobilitätsrechte gewähren. An dieser Stelle sei erneut auf jene sehr mobilen, sehr reichen Industriestaaten der OECD-Gemeinschaft verwiesen, die bestimmte Formen von Mobilität besonders hart bekämpfen oder in äußerst prekäre Bedingungen zwingen, während ihre eigenen Staatsbürger/innen in nahezu alle Länder der Welt visumfrei einreisen können; insbesondere im Fall der Mitgliedstaaten der Europäischen Union. ${ }^{6}$ Im Gegensatz zu dieser mobilen Elite ist ein Großteil der Menschheit ausgeschlossen vom Freizügigkeitsversprechen des Schengenraums und dem Rest der westlichen Hemisphäre.

Mit dem Konzept der »Birthright Lottery« (Shachar 2009) knüpft Ayelet Shachar an das normative Verständnis des Rechts auf Bewegungsfreiheit als Recht auf Selbstbestimmung an. In ihrer kritischen Analyse zeitgenössischer Staatsangehörigkeitsrechte legt Shachar dar, dass das grundlegende Zugehörigkeitsprinzip der Staatsbürgerschaft durch Geburt die Mehrheit der Menschen weltweit von der Möglichkeit ausschließt, jenseits des $\mathrm{Zu}$ falls ihrer Geburt Mitglied einer politischen Gemeinschaft zu werden. Betrachten wir das globale Mobilitätsregime im Rückgriff auf Shachar, sehen wir, dass Grenzen mehr sind als nur territoriale Marker eines internationalen Staatensystems - sie sind ordnende Kraft von Berechtigungsstrukturen, deren ungleiches Verteilungsprinzip sich durch eine nostalgische Vorstellung von Staatsbürgerschaft legitimiert, die politische Gemeinschaften als räumlich gebunden und in ihrer nationalen Verbundenheit als unveränderlich erträumt (vgl. ebd. 1-7).

6 Zur europäischen Migrations- und Grenzpolitik, siehe zum Beispiel Tsoukala 2005; Huysmans 2006; van Munster 2009; van Houtum 2010; Toğral 2011; Andersson 2014; Lazaridis/Wadia 2015 und Schulze Wessel 2017. 
Gehen wir jedoch zurück zu der Tatsache, dass für eigentlich alle Krisenregionen der Welt der Zugang zu legalen und somit sicheren Ausreisewegen durch die Visumpflicht beschränkt ist, müssen wir die Frage stellen, ob und inwiefern ein solcher Ausschlussmechanismus überhaupt zu rechtfertigen ist. Diese Perspektive nimmt die Verpflichtungen liberaler Demokratien im Rahmen internationaler Menschen- und Flüchtlingsrechtsabkommen in den Blick.

Da nach dem zeitgenössisch vorherrschenden Verständnis staatlicher Zuständigkeitsbereiche für Flüchtende keinerlei Anspruch auf ein Asylverfahren besteht, solange sie sich außerhalb des Staatsgebiets des ersuchten Aufnahmelands befinden, kann eine restriktive Visumpolitik den Zugang zum Recht auf Asyl erschweren oder sogar verhindern (vgl. Laube 2017: 57-58). In dieser kritischen Lesart stellt die Auferlegung der Visumpflicht für liberale Demokratien eine Lösungsstrategie dar für das »Kontrolldilemma [...], welches aus dem Interesse an restriktiven Politiken gegenüber unerwünschten Grenzüberschreitern einerseits und den eigenen rechtlichen Verpflichtungen gegenüber genau diesen Personen andererseits resultiert« (Laube 2010: 1). Wandernde, die kein Visum erhalten, müssen sich auf Wege jenseits der legalen Einreise- und Einwanderungsbestimmungen begeben.

Im nächsten Schritt argumentiert dieser Beitrag, dass für manche Menschen keine legalen und somit sicheren Wege nach Europa vorgesehen sind (vgl. Schulze Wessel 2017: 136). Das Massensterben vor den europäischen Außengrenzen macht auf drastische Art und Weise deutlich, welche Gefahren für jene Menschen einhergehen mit dem Versuch dennoch Zugang zu finden zum Geltungsbereich europäischen Rechts: »Wenn du nicht den richtigen Pass hast, ist dein Leben an der Grenze zu Ende. Du bist ein Problem, ein Risiko, ein Ärgernis, deinetwegen werden Barrieren errichtet« (Scego 07.07.2016).

Eben diese hierarchisch geschaffene Unterscheidung »zwischen offiziell anerkannten, rechtlich gesicherten Wegen zum Zielland und den unregulierten Wegen von Migranten, die keine offiziellen Einreisepapiere besitzen« (Schulze Wessel 2017: 81) markiert den Bruch im Narrativ des europäischen >Raums der Freiheit, der Sicherheit und des Rechts $\measuredangle$. 


\section{DAS SCHENGENVISUM UND DIE AUSHÖHLUNG DES EUROPÄISCHEN WERTEKANONS}

Eingebettet in die Vorstellung einer grenzenlosen Welt stehen die Europäische Union und das Konzept von Schengen als ein >Raum der Freiheit, der Sicherheit und des Rechts beispielhaft für ein Recht auf Freizügigkeit, das über territoriale Grenzen hinaus von Geltung ist. Bis heute gilt der freie Personenverkehr über die europäischen Binnengrenzen hinweg als »eine der größten Errungenschaften der Union « ${ }^{7}$ und erfährt unter dem Vorzeichen europäischer Integration eine ideelle Vorrangstellung. Wie die Analyse des europäischen Visumregimes in diesem Kapitel zeigt, folgt aus der Europäisierung der nationalen Grenzen der Mitgliedstaaten jedoch nicht der Bedeutungsverlust der Grenzkontrolle, sondern eine räumliche Verlagerung der Entscheidung zwischen Grenzöffnung und Grenzschließung.

Wer nach Europa auf legalem Weg aufbrechen darf und wer nicht, reguliert die europäische Visumpolitik entlang eines Kontinuums abgestufter Mobilitätsrechte. Zwei einheitliche Listen benennen sowohl die Nationen, deren Staatsbürger/innen visumfrei nach Schengen einreisen dürfen, als auch die Nationen, deren Staatsbürger/innen erst einen Visumantrag stellen müssen - die sogenannte Negativ- und Positivliste. Staatsbürger/innen der Länder auf der Positivliste haben die Möglichkeit visumfrei für bis zu 90 Tage in die Europäische Union einzureisen. Hingegen müssen Staatsbürger/innen der Länder auf der Negativliste für den Erhalt eines Visums für Kurzaufenthalte hohe Anforderungen innerhalb eines Antragsprozess erfüllen, dessen Verfahren und Voraussetzungen durch den Visakodex ${ }^{8}$ festgelegt sind. Des Weiteren gibt es für Konsulate und andere Behörden mit Zuständigkeit für die Vergabe des Schengenvisum ein Handbuch über die Anwendung des Visakodex, ${ }^{9}$ das $"$ praktische Anweisungen

7 Siehe Amtsblatt der Europäischen Gemeinschaften, OJ L 295 vom 06.11.2013, S. 1.

8 Siehe Verordnung (EG) Nr. 810/2009 vom 13.07.2009, OJ L 243 vom 15.09.2009, S. 1, letzte Änderung durch Verordnung (EU) Nr. 610/2013 vom 26.06.2013, OJ L 182 vom 29.06.2013, S. 1.

9 Siehe $>$ Konsolidierte Fassung des Handbuchs für die Bearbeitung von Visumanträgen und die Änderung von bereits erteilten Visa auf der Grundlage des Be- 
(Leitlinien, bewährte Verfahren und Empfehlungen) $\ll^{10}$ enthält. Während Visakodex und Visahandbuch sowohl vorgeben in welcher Form der Visumantrag erfolgen als auch geprüft werden muss, benennt die Verordnung (EG) 539/2001 die Drittländer der Visumlisten und die Kriterien, anhand welcher Drittländer der Visumpflicht unterstellt oder von der Visumpflicht befreit werden.

Nach der Ergänzung der Verordnung (EG) 539/2001 durch Verordnung (EU) 509/2014 werden die Drittländer, deren Staatsangehörige der Visumpflicht unterliegen oder von der Visumpflicht befreit sind, bestimmt

»auf der Grundlage einer fallweise gewichteten Bewertung mehrerer Kriterien, die unter anderem die illegale Einwanderung, die öffentliche Ordnung und Sicherheit, die wirtschaftlichen Vorteile, insbesondere in Bezug auf Tourismus und Außenhandel, sowie die Außenbeziehungen der Union zu den entsprechenden Drittländern betreffen, wobei insbesondere Erwägungen in Bezug auf die Menschenrechte und die Grundfreiheiten und die regionale Kohärenz und der Grundsatz der Gegenseitigkeit zu berücksichtigen sind «. ${ }^{11}$

Wichtiges Kernstück der Verordnung (EG) 539/2001 sind die Listen der Drittländer, deren Staatsangehörige beim Überqueren der Außengrenzen im Besitz eines Visums sein müssen, sowie der Drittländer, deren Staatsangehörige von dieser Pflicht befreit sind. Im Jahr 2018 unterliegen 101 Länder der Visumpflicht im Gegensatz zu 58 Ländern, die Visumfreiheit genieBen. ${ }^{12}$ Bemerkenswert ist angesichts dieser zahlenmäßigen Diskrepanz, dass der Rechtsakt zur Aufstellung der Visumlisten zwar auf die »fallweise

schlusses K(2010) 1620 endgültig der Kommission vom 19.03.2010, geändert durch Durchführungsbeschluss K(2011) 5501 endgültig der Kommission vom 4.8.2011.

10 Siehe ebd. S. 16.

11 Siehe Verordnung (EU) 509/2014 vom 15.05.2014, OJ L 149 vom 20.05.2014, S. 69, Artikel -1 als vorangestellte Ergänzung zu Artikel 1 der Verordnung (EG) 539/2001 vom 15.03.2001, OJ L 81 vom 21.03.2001, S. 1; eigene Hervorhebung.

12 Siehe Annex I und II. 
gerichtete[n] Bewertung mehrerer Kriterien ${ }^{13}{ }^{3}$ verweist, doch sich jenseits dieser Aussage in den sonst stets transparent gehaltenen Kommunikationskanälen der EU keine detaillierten Informationen darüber finden lassen, nach welchem Verfahren die Visumlisten erstellt wurden und warum die Aufteilung der Länder so und nicht anders erfolgte. Auf welche Liste ein Land verwiesen wurde, hat jedoch weitreichende Konsequenzen (vgl. van Houtum 2010: 964). Ist eine Nation vom Visumzwang befreit, steht den Staatsbürger/innen die selbstbestimmte Entscheidung nach Europa einzureisen grundsätzlich offen. Sie genießen bevorzugtes Vertrauen und benötigen für die Einreise im Rahmen eines Kurzzeitaufenthalts zunächst nicht mehr als ihren Pass. Die Kontrollfunktion der Schengengrenze spüren sie erst, wenn sie das europäische Territorium erreichen. Im Umkehrschluss erfahren Staatsbürger/innen visumpflichtiger Staaten die Selektivität der Schengengrenze bereits bevor sie überhaupt den Aufbruchsort verlassen. Der Visumzwang stellt sie als Teil einer Nation unter Verdacht, ein erhöhtes Risiko für das Zielland darzustellen. So gelten `Bona-fide〈-Reisende aus Staaten wie Australien, den USA oder Kanada als vertrauenswürdig, während >Mala-fide〈-Reisende aus Ländern wie dem Iran, Irak oder Syrien grundsätzlich für ein Mehr an Kontrolle herausgegriffen werden. Dabei erfolgt die Risikozuschreibung nicht einzeln und basierend auf individuellem Verhalten, sondern präventiv für alle Angehörigen eines Staates. Diese Vorgehensweise erinnert an einen Profiling-Ansatz, der Nationalität als Gruppeneigenschaft betrachtet und vom vermeintlichen Bedrohungspotential bestimmter Nationalitäten auf eine allgemeine Notwendigkeit von Einzelfallüberprüfungen für alle Angehörigen eines Staates schließt (vgl. Guild 2001: 34). Nur diejenigen >Mala-fide〈-Reisenden erhalten ein Visum, die im Antragsverfahren ausreichend darstellen können, dass sie entgegen der Negativkategorisierung ihrer nationalen Herkunft bestimmte Mobilitätskriterien erfüllen. Erst im Antragsverfahren können Staatsangehörige der Länder auf der Negativliste den ihnen gegenüber geäußerten Generalverdacht widerlegen, wobei den Antragsstellenden die Beweislast obliegt. Bereits die kostspieligen und sehr zeitaufwendigen Anforderungen des

13 Siehe Verordnung (EU) 509/2014 vom 15.05.2014, OJ L 149 vom 20.05.2014, S. 69, Artikel -1 als vorangestellte Ergänzung zu Artikel 1 der Verordnung (EG) 539/2001 vom 15.03.2011, OJ L 81 vom 21.03.2001, S. 1. 
Visumantrags und der Terminvergabe beschränken daher, wer die Chance hat sich dem Auswahlprozess zu stellen. Darüber hinaus müssen Visumbeantragende im Rahmen des Vergabeverfahrens verschiedene Nachweise erbringen, insbesondere über Einkommen, Wohnort, Familienstatus, Reisezweck und Rückkehrabsicht (vgl. Mau 2010: 344-345). Wer ein Visum beantragen muss, profitiert daher in erster Linie von ausreichendem ökonomischen Kapital und einem Persönlichkeitsprofil, das der europäischen Vorstellung >erwünschter Mobilität entspricht - und dennoch gibt es keine Garantie auf die Gewährleistung des Visumantrags. Die grundsätzliche Einschränkung legaler Einreisewege für eine bestimmte Gruppe von Nationen zeichnet so eine gefährliche Umkehr der rechtsstaatlichen Unschuldsvermutung nach.

Wie Henk van Houtum herausstellt, lauteten die Titel der sogenannten Negativ- und Positivliste zuvor \Schengener schwarze Liste gener weiße Lister (vgl. van Houtum 2010: 964). Angesichts der geographischen Verteilung der Drittstaaten, deren Staatsangehörige der Visumpflicht unterstehen, unterstreicht die Bezeichnung der Negativliste als >Schengener schwarze Liste die fragwürdige Zuordnung von Identitätszuschreibungen des >Weiß-Seins $\triangleleft$ und des `Schwarz-Seins $\triangleleft$ in Abgrenzung der Staaten des globalen Nordens und des globalen Südens. Dies wirft die Frage auf, inwiefern in dieser Aufteilung rassistische Narrative reproduziert werden, die Menschen aufgrund ihrer Staatsbürgerschaft entlang von Kriterien wie Wohlstand, Ethnie und Religion stigmatisieren (vgl. Kirsch 2017). Fast alle Staaten in Afrika, Asien und im Nahen Osten befinden sich auf der >Schengener schwarzen Liste - darunter insbesondere Staaten, die von Armut und Ressourcenmangel sowie Konflikten und Kriegen betroffen sind. Beachtenswerte Ausnahmen sind zum Beispiel die Länder Israel, Brunei Darussalam, Japan, Süd-Korea, Malaysia, Singapur sowie die Vereinigten Arabischen Emirate und die Sonderverwaltungsregionen der Volksrepublik China, Hongkong und Macau. Auf der >weißen Liste Brunei Darussalam und die Vereinigten Arabischen Emiraten die einzigen Länder mit einer mehrheitlich muslimischen Bevölkerung. Im Vergleich zu ihren regionalen Nachbarn lässt sich am Beispiel beider Länder besonders deutlich das starke Gewicht der Kriterien der »wirtschaftlichen Vorteile, insbesondere in Bezug auf Tourismus und Außenhandel, sowie [der] Au- 
ßenbeziehungen der Union zu den entsprechenden Drittländern $«{ }^{14}$ vermuten. Ähnlich scheint die Einteilung der meisten Länder Lateinamerikas auf der weißen Liste begründet, darunter zum Beispiel Venezuela, Kolumbien und Mexiko. So erscheint die Umbenennung der \Schengener weißen Liste und >Schengener schwarzen Liste $<$ in Positiv- und Negativliste jedoch nur als zweifelhafter Rebranding-Versuch für die Imageaufbesserung eines unveränderten Exklusivclubs. Insbesondere angesichts der Tatsache, dass keine Informationen zur Einsicht vorliegen über die Erstellung der ursprünglichen Aufteilung der Drittländer auf die Visumlisten der Verordnung (EG) 539/2001, ist die offensichtliche Diskriminierung von Menschen des globalen Südens ein zentrales Argument für die Entwicklung von objektiveren Maßstäben und transparenten Bewertungsverfahren für die Entscheidung über die Aufhebung oder Auferlegung der Visumpflicht (vgl. Peers 2011: 294).

Die Verordnung über die gemeinsamen Visumlisten macht anhand der zuvor aufgeführten Kriterien deutlich, dass aus europäischer Perspektive von Staatsbürger/innen der Länder auf der Negativliste anzunehmen ist, dass sie aufgrund ihrer Nationalität mit einer höheren Wahrscheinlichkeit gegen geltendes europäisches Einreise-, Aufenthalts- und Kriminalrecht verstoßen werden. Insbesondere zeigt die an den Beginn der Aufzählung gestellte Aneinanderreihung der Kriterien der >illegalen` Einwanderung sowie der öffentlichen Ordnung und Sicherheit, dass ein möglicher Verstoß gegen das europäische Einreise- und Aufenthaltsrecht rhetorisch mit dem Bedrohungspotential eines kriminellen Verbrechens verknüpft und gleichgesetzt wird. Aber erst die Illegalisierung der Bewegungsfreiheit bestimmter Gruppen von Menschen ruft eine Realität ins Leben, in der Menschen durch die selektive Einschränkung von legalen Einreisewegen im Umkehrschluss gezwungen sind auf >illegalen Wegen nach Europa aufzubrechen. Durch die Kriminalisierung undokumentierter Wanderungen entsteht ein Teufelskreis, der den Nährboden für genau jene Angstbilder von >Flüchtlingsströmen`schafft, welche wiederum zur Rechtfertigung einer restrikti-

14 Siehe Verordnung (EU) 509/2014 vom 15.05.2014, OJ L 149 vom 20.05.2014, S. 69, Artikel -1 als vorangestellte Ergänzung zu Artikel 1 der Verordnung (EG) 539/2001 vom 15.03.2011, OJ L 81 vom 21.03.2001, S. 1. 
ven Grenzpolitik gegenüber genau diesen Gruppen von Menschen herangezogen werden.

\section{Abbildung 1: Das europäische Visumregime}

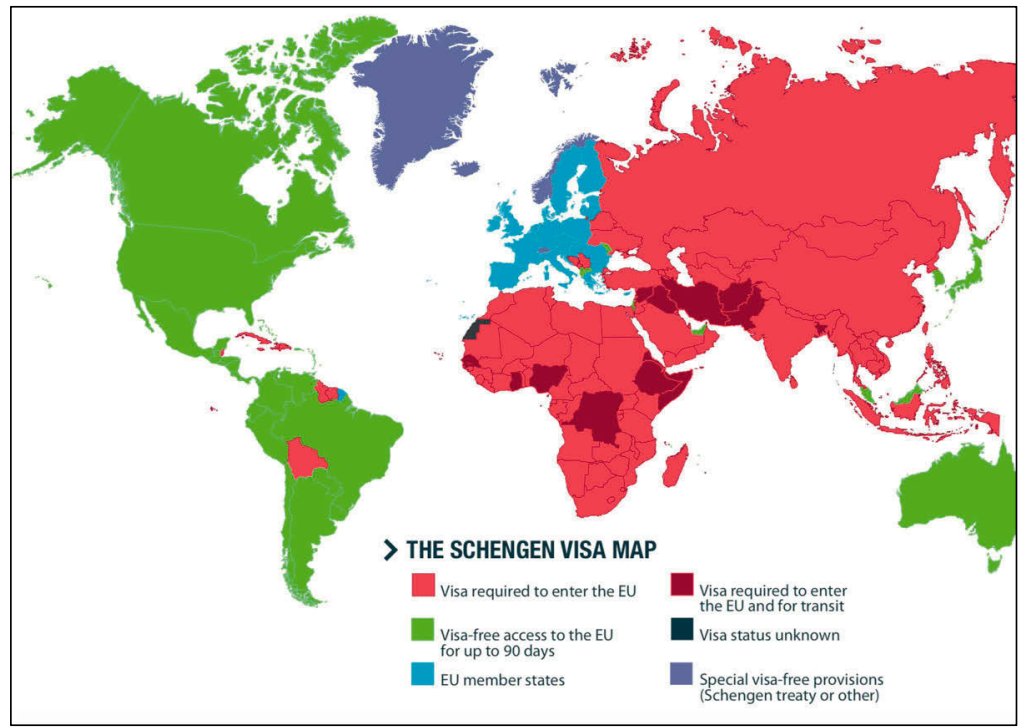

Quelle: Kirsch 2017.

Das Netzwerk UNITED for Intercultural Action verdeutlicht durch die Veröffentlichung der Opferzahlen undokumentierter Wanderungen die lebensgefährlichen Konsequenzen der selektiven Einschränkung legaler Wege in die Europäische Union. Die detaillierte Auflistung des Berichts »Death by Policy - Time for Change!« (UNITED 2017) zählt 33.305 Menschen, die bis zum 15. Juni 2017 auf dem Weg nach Europa ums Leben gekommen sind. Ähnlich geht die Projektseite The Migrants' Files von über 30.000 Toten seit dem Jahr 2000 aus (vgl. The Migrants' Files 2016). Trotz des für die Europäische Union konstitutiven Bekenntnis zu Freiheit, Demokratie, Gleichheit, Rechtsstaatlichkeit und der Wahrung der Menschenrechte sind die europäischen Außengrenzen zu den tödlichsten Grenzen der Welt geworden (vgl. Schulze Wessel 2017: 12). Dieses Massensterben darf nicht getrennt betrachtet werden von den politischen Entscheidungen, die dazu geführt haben, dass gerade diejenigen Menschen auf immer gefährli- 
chere Routen gezwungen werden, die Schutz benötigen und in Europa suchen.

Die Entscheidung des Europäischen Gerichtshof für Menschenrechte (EuGH) vom 07. März 2017 in der Rechtssache C-638/16 PPU über das Fehlen einer Verpflichtung für die Erteilung eines humanitären Visums aus humanitären Gründen oder aufgrund internationaler Verpflichtungen verschärft noch stärker den Eindruck, dass der europäische Kampf gegen >illegaleく Migration keinen Schutz für Flüchtende jenseits des europäischen Territoriums kennt. Das Urteil betrifft die Klage einer syrischen Familie, die im Oktober 2016 in der belgischen Botschaft im libanesischen Beirut einen Visumantrag mit begrenzter räumlicher Gültigkeit gestellt hatte, um für einen Asylantrag nach Belgien zu gelangen. Laut Artikel 19 des Visakodex kann ein Visumantrag, der die Kriterien der Zulässigkeit nicht erfüllt, dennoch »aus humanitären Gründen oder aus Gründen des nationalen Interesses als zulässig betrachtet werden «. ${ }^{15}$ Des Weiteren legt Artikel 25 fest, dass ein Visum mit räumlich beschränkter Gültigkeit im Ausnahmefall erteilt werden kann, »wenn der betreffende Mitgliedstaat es aus humanitären Gründen, aus Gründen des nationalen Interesses oder aufgrund internationaler Verpflichtungen für erforderlich hält«. ${ }^{16}$ Für den Antrag eines Visums aufgrund humanitärer Gründe oder aufgrund internationaler Verpflichtungen gibt es nach europäischem Recht jedoch kein eigenständiges Verfahren, sondern das Vorliegen eines solchen Ausnahmefalls muss im Rahmen eines regulären Visumantrags für einen Kurzaufenthalt von bis zu 90 Tagen festgestellt werden. Dennoch schlussfolgert der EuGH in seinem Urteil, dass das Gesuch eines humanitären Visum für einen Antrag auf Asyl keinen Visumantrag für einen Kurzzeitaufenthalt darstellt, sondern auf Dauer ausgelegt ist und deshalb nicht unter die Bestimmungen des Visakodex fällt. Die Bestimmungen für die Erteilung eines Langzeitvisum sind allein Gegenstand der nationalen Gesetzgebung. Daher fällt die Frage, ob die EU-Mitgliedstaaten zur Erteilung eines humanitären Visums verpflichtet sind, in der Auslegung des humanitären Visum als Langzeitvisum au-

15 Siehe Verordnung (EG) Nr. 810/2009 vom 13.07.2009, OJ L 243 vom 15.09.2009, letzte Änderung durch Verordnung (EU) Nr.610/2013 vom 26.06.2013, OJ L 182 vom 29.06.2013, S. 20.

16 Siehe ebd., S. 23-24. 
ßerhalb des Kompetenzbereichs des EuGH (vgl. Spijkerboer 2017: 225226). Wäre der EuGH in seinem Urteil der Argumentation des Generalanwalts Paolo Mengozzi gefolgt, so wären die EU-Mitgliedstaaten verpflichtet gewesen es zu ermöglichen, einen Antrag auf internationalen Schutz bei den Vertretungen der Mitgliedstaaten außerhalb des Schengenraums zu stellen. Mengozzi argumentierte, dass die syrische Familie einen Antrag auf ein Kurzzeitvisum gestellt hatte und der Rückgriff auf die Ausnahmeregelungen des Visakodex es demnach erlaubt, den Antrag auf ein solches Kurzzeitvisum trotz begründeter Zweifel an der Rückkehrabsicht als zulässig zu betrachten. Hierfür führte Mengozzi an, dass der Verbleib der Antragsstellenden in der Europäischen Union über die Einreise im Rahmen des Kurzzeitvisum hinaus unter den rechtlichen Status als Asylsuchende fällt. Mengozzi argumentierte daher, dass unter dem Visakodex für die EUMitgliedstaaten eine Verpflichtung zur Erteilung eines humanitären Visum besteht, sobald Antragsstellende durch die Nichterteilung eines humanitären Visums in ihrem Leben und ihrer Freiheit bedroht sind im Sinne des internationalen Rechts und des Unionsrechts (vgl. ebd.). Wie Thomas Spijkerboer feststellt, ist die Entscheidung des EuGH jedoch insofern kaum überraschend, als dass die belgische Regierung, die Regierung von 13 weiteren EU-Mitgliedstaaten und die Europäische Kommission sich im Vorhinein gegen eine solche Auslegung gestellt hatten (vgl. ebd. 227). Im Licht des europäischen Wertekanon scheint es mehr als zynisch, dass offenbar alles daran gesetzt wird, um zu verhindern, dass Schutzsuchende den Schengenraum erreichen.

Gegründet auf »die Achtung der Menschenwürde, Freiheit, Demokratie, Gleichheit, Rechtsstaatlichkeit und die Wahrung der Menschenrechte einschließlich der Rechte der Personen, die Minderheiten angehören « ${ }^{17}$, bezieht die Europäische Union aus ihrer Selbstverpflichtung als Wertegemeinschaft ihre politische Legitimität. Weder die Europäische Menschenrechtskonvention, noch die EU-Grundrechtscharta oder die Genfer Flüchtlingskonvention sehen vor, dass die Einreise und Aufnahme von Schutzsuchenden de jure entlang ökonomischer, politischer und/oder kultureller Merkmale eingeschränkt werden kann. Wie die europäische Visumpolitik eindrücklich belegt, erfüllt die räumliche Verlagerung der Grenze jedoch de

17 Siehe AEUV in der Version von Lissabon, Artikel 2. 
facto genau diese Selektionsfunktion. Gleichzeitig wird die Unterscheidung zwischen legalen und sillegalen` Wanderungen als Rechtfertigung für den Rechtsvorenthalt angeführt. Das Label der >Illegalität bedient einen vermeintlich notwendigen Sicherheitsimperativ, der legitimiert, »dass kaum mehr rechtlich überprüft wird, ob diejenigen, die jenseits der Einwanderungsregeln in [europäisches] Rechtsgebiet gelangen oder gelangen wollen, als Flüchtlinge im Sinne der Genfer Konvention gelten können oder nicht« (Schulze Wessel 2017: 82). Verstehen wir die europäische Migrations- und Grenzkontrollpolitik als eine Politik der Kategorisierung, sehen wir, dass neben der Unterscheidung zwischen legalen und >illegalen` Wegen nach Europa jede andere Differenzierung zunehmend an Bedeutung verliert. ${ }^{18}$ Wer im falschen Land geboren wird, hat kaum Möglichkeiten das globale Nord-Süd-Gefälle zu überwinden.

\section{FAZIT}

Die kritische Lesart des globalen Visumregimes in diesem Beitrag zeigt auf, dass insbesondere die Staatsbürger/innen aus Ländern des globalen Nordens ein großes Maß an Reisefreiheit genießen. Hingegen müssen Staatsbürger/innen aus Ländern des globalen Südens ungleich viel höhere Barrieren für ein Recht auf Bewegungsfreiheit überwinden. In einer Welt, in der Lebenschancen sehr ungleich verteilt sind, wirft die Beschneidung der individuellen Bewegungsfreiheit entlang sozioökonomischer und rassistischer Hierarchien dringende Fragen auf nach globaler Gerechtigkeit - und der Universalität des Menschseins. Besonders prekär sind die Mobilitätsbeschränkungen durch Visumpflicht vor dem Hintergrund der Tatsache, dass die Staatsbürger/innen mit dem geringsten Umfang an Ausreisemöglichkeiten aus jenen Ländern stammen, die am stärksten von Konflikten und Leid bedroht sind. Zwar schützt das internationale Flüchtlingsrecht und das Völkerrecht auf dem Gebiet der Menschenrechte vor der Zurückweisung Schutzsuchender in ein Gebiet, in dem Verfolgung droht oder die Gefahr der Folter sowie anderer schwerwiegender Verletzungen der Menschen-

18 Zum innerdeutschen Migrationsdiskurs und der Kategorie des >Wirtschaftsflüchtlings`, siehe den Beitrag von Kania in diesem Band. 
rechte besteht - wer aber die territoriale Grenzlinie niemals erreicht, befindet sich, so das staatliche Rückzugsargument, außerhalb des staatlichen Verantwortungsbereiches. ${ }^{19}$ In den »Grenzfiguren« (Schulze Wessel 2017) der Mobilitätskluft kristallisiert sich so das Paradox im Kern territorial organisierter, liberal-demokratischer Nationalstaaten »zwischen der Universalität der Normen und dem Grenzen schaffenden Anspruch auf demokratische Selbstbestimmung « (ebd. 209).

Liberale Demokratien binden sich zunehmend an internationale Normen und Wertvorstellungen. Sie verpflichten sich in besonderem Maße dem Schutz des Individuums, auch gegenüber Nicht-Staatsbürger/innen. Dennoch verbleiben sie nach dem Prinzip territorialer Souveränität als zentrale Akteurinnen der Umsetzung der verschiedenen Abkommen und Konventionen innerhalb ihres eigenen Hoheitsgebiets. Verpflichten sich liberale Demokratien dem internationalen Menschen- und Flüchtlingsrecht, gewährleisten jedoch nicht jeder Person dasselbe Maß an Rechtsschutz, bricht staatliches Handeln mit dem selbstauferlegten Wertekanon. Gleichzeitig legitimiert das vorherrschende zeitgenössische Verständnis absoluter nationalstaatlicher Souveränität jedoch, dass liberal-demokratische Nationalstaaten den Zugang zu ihrem Territorium regulieren und darüber entscheiden, wer sich unter welchen Bedingungen innerhalb ihrer Grenzen bewegen darf. Solange territorial begrenzte Nationalstaaten die Verantwortung tragen konkrete Rechte zu gewähren, besteht deshalb die Gefahr, dass NichtStaatsbürger/innen vom Zugang zu diesen Rechten ausgeschlossen werden. Denn um das völkerrechtlich verankerte Recht auf Non-Refoulement - auf Nicht-Zurückweisung - geltend machen zu können, müssen Schutzsuchende die territoriale Grenze des ersuchten Staates zuerst überhaupt erreichen. $^{20}$

Das Ineinandergreifen von Visumbestimmungen und den sogenannten >Carrier Sanctions` führt bei Nichtvorhandensein eines legalen Einreiseund Aufenthaltstitels entweder zur vollständigen Immobilisierung oder

19 Zur politischen Praxis des völkerrechtlich geschützten Verantwortungsbereichs im internationalen Seerecht, siehe den Beitrag von Pichl/Tohidipur in diesem Band.

20 Zur rhetorischen Aufweichung und Verschiebung von vermeintlich klar definierten territorialen Grenzlinien, siehe den Beitrag von Känner in diesem Band. 
erzwingt den Versuch undokumentiert und unter Lebensgefahr in das ersuchte Land einzureisen. Jedes Individuum, das sich dennoch der Hierarchie der Grenze widersetzt und undokumentiert die Reise beginnt, bewegt sich schon im Moment des Aufbrechens außerhalb des Bereichs des im Zielland geltenden Rechts: »Der Zugang zum Recht ist somit der Differenzierung zwischen legalen, geordneten Wanderungen und illegalen, ungeordneten Wanderungen unterworfen« (ebd. 178). Jeder Verstoß gegen das globale Mobilitätsregime trägt so das kriminelle Stigma der >Illegalitätく. Durch die Logik dieser Unterscheidung stuft der globale Norden die Universalität des Menschen- und Flüchtlingsrechts zu einem Privileg der westlichen Welt ab. Gleichzeitig ist die Illegalisierung und Kriminalisierung bestimmter Wanderungsbewegungen die Voraussetzung für »die Umgehung und komplette Aushebelung rechtlicher Standards« (Schulze Wessel 2017: 182). Erst die Verlagerung der Grenzkontrolle als eine Strategie des systematischen Rechtsvorenthalts macht sichtbar, dass weniger das Warum der Wanderung als ihr Ursprungsort bestimmt, welchen Wanderungen bereits mit dem Aufbruch jegliche Berechtigung abgesprochen wird. Nicht der territoriale Verlauf der Grenzlinie entscheidet darüber, wer die Grenze zu passieren vermag. Wer auf >illegalem` Weg aufbricht, reist im Zeichen des Fremden, des Anderen, des Bedrohlichen.

So ist die Grenze mehr als eine Linie - sie ist eine Hierarchie von Privilegien, die Menschen und ihr Recht auf Leben und Freiheit in Legalitätskategorien verarbeitet. Die Grenze ist Scheideweg jener, die sie einfach passieren, und der anderen, die sie vielleicht erreichen werden. 


\section{LITERATUR}

Andersson, Ruben, 2014: Illegality Inc.: Clandestine Migration and the Business of Bordering Europe. University of California Press.

Angeli, Oliviero, 2011: Das Recht auf Einwanderung und das Recht auf Ausschluss, in: Zeitschrift für Politische Theorie, 2, 2, S. 171-184.

Bigo, Didier/Guild, Elspeth, 2005: Policing at a Distance. Schengen Visa Policies, in: Bigo, D./Guild, E. (Hg.), Controlling Frontiers: Free Movement Into and Within Europe, S. 233-262. Routledge.

Bréville, Benoît, 2014: Der richtige Pass, in: Le Monde Diplomatique, 14.02.2014, unter: https://monde-diplomatique.de/artikel/!394721 [Zuletzt aufgerufen am 16.03.2018].

Chojnacki, Sven/Paping, Lisa, 2016: Migration im Spannungsfeld von Raumwandel, Kontrolle und Gewalt. Herausforderungen für eine kritische Friedensforschung, in: S+F, 34, 1, S. 20-28.

Gold, Judith/El-Ashram, Ahmed, 2015: A Passport of Convenience, in: Finance \& Development, 52, 4, S. 48-51.

Guild, Elspeth, 2001: Moving the Borders of Europe. The Inaugural Lecture Delivered During the Official Ceremony on the Occasion of the Assumption of the Professorship of the CPO Wisselleerstoel at the University of Nijmegen, the Stichting Steunfonds Juridisch (Post) Doctoraal Onderwijs on 30 May 2001, unter: https://cmr.jur.ru.nl/ cmr/docs/oratie.eg.pdf [Zuletzt aufgerufen am 01.03.2018].

Henley\&Partners, 2017: Visa Restrictions Index 2017, unter: https://henleyglobal.com/files/download/hvri/HP_Visa_Restrictions_In dex_170301.pdf [Zuletzt aufgerufen am 18.11.2018].

Henley\&Partners, 2018: Citizenship by Investment, unter: https:/www. henleyglobal.com/citizenship-planning/ [Zuletzt aufgerufen am 18.11. 2018].

Hess, Sabine/Kasparek, Bernd/Schwertl, Maria/Sontowski, Simon, 2015: Europäisches Grenzregime. Einleitung zur ersten Ausgabe, in: movements. Journal für kritische Migrations- und Grenzregimeforschung, 1, 1, S. 1-7.

Huysmans, Jef, 2006: The Politics of Insecurity: Fear, Migration and Asylum in the EU. Routledge. 
Kirsch, Thea, 2017: Unequal Im/Mobilities. Why Do Visa Policies Exclude Certain Groups of People But Not Others? Posterbeitrag zur studentischen Konferenz >Herkunft Zukunft an der HTW Berlin im Januar 2017, unter: https://www.htw-berlin.de/files/Presse/Herkunft_Zukunft /Herkunft_Zukunft_Thea_Kirsch.pdf [Zuletzt aufgerufen am 18.11.2018].

Laube, Lena, 2010: Wohin mit der Grenze? Die räumliche Flexibilisierung von Grenzkontrolle in vergleichender Perspektive. TranState Working Papers, 112. Sfb $597 »$ Staatlichkeit im Wandel«.

Laube, Lena, 2013: Grenzkontrollen jenseits nationaler Territorien. Die Steuerung globaler Mobilität durch liberale Staaten. Campus.

Laube, Lena, 2017: >Asyl fängt ja erst an, wenn er [der Flüchtling] wirklich hier ist. Der Wandel europäischer Visapolitik und seine Implikationen für den Zugang zu Asylverfahren in der EU, in: Lahusen, C./Schneider, S. (Hg.), Asyl verwalten. Theoretische Perspektiven und empirische Befunde zur bürokratischen Bearbeitung eines gesellschaftlichen Problems, S. 55-78. transcript.

Lazaridis, Gabriella/Wadia, Khursheed (Hg.), 2015: The Securitisation of Migration in the EU: Debates since 9/11. Palgrave MacMillan.

Lori, Noora A., 2017: Statelessness, >In-Between`Statuses, and Precarious Citizenship, in: Shachar, A. et al. (Hg.), The Oxford Handbook of Citizenship, S. 743-766. Oxford University Press.

Mau, Steffen, 2010: Mobility Citizenship, Inequality, and the Liberal State: The Case of Visa Policies, in: International Political Sociology, 4, 4, S. 339-361.

Mau, Steffen, 2011: Grenzen als Sortiermaschinen, in: Kleger, H. (Hg.), Umstrittene Bürgerschaft: Grenzen, Identitäten und Konflikte, S. 72-84. Universitätsverlag Potsdam.

Mau, Steffen/Brabandt, Heike, 2011: Visumpolitik und die Regulierung globaler Mobilität. Ein Vergleich dreier OECD Länder, in: Zeitschrift für Soziologie, 40, 1, S. 3-23.

Mau, Steffen/Brabandt, Heike/Laube, Lena/Roos, Christof, 2012: Liberal States and the Freedom of Movement: Selective Borders, Unequal Mobility. Basingstoke.

Mau, Steffen/Gülzau, Fabian/Laube, Lena/Zaun, Natascha, 2015: The Global Mobility Divide: How Visa Policies Have Evolved over Time, in: Journal of Ethnic and Migration Studies, 41, 8, S. 1192-1213. 
McKinsey Global Institute, 2016: Digital Globalization. The New Era of Global Flows. Unter: https://www.mckinsey.com/ /media/McKinsey/ Business\%20Functions/McKinsey\%20Digital/Our\%20Insights/Digital \%20globalization\%20The\%20new\%20era\%20of\%20global\%20flows/ MGI-Digital-globalization-Full-report.ashx [Zuletzt aufgerufen am 18.11.2018].

Peers, Steve, 2011: EU Justice and Home Affairs Law. Oxford.

Rodenhäuser, Tilman, 2014: Carrier Sanctions and the Privatization of Immigration Control, in: International Journal of Refugee Law, 26, 2, S. 223-247.

Scego, Igiaba, 2016: Ungleiche Pässe, in: Le Monde Diplomatique, 07.07.2016, unter https://monde-diplomatique.de/artikel/!5310325 [Zuletzt aufgerufen am 18.03.2018].

Schulze Wessel, Julia, 2016: On Border Subjects: Rethinking the Figure of the Refugee and the Undocumented Migrant, in: Constellations, 23, 1, S. 46-57.

Schulze Wessel, Julia, 2017: Grenzfiguren. Zur politische Theorie des Flüchtlings. transcript.

Shachar, Ayelet, 2009: The Birthright Lottery. Citizenship and Inequality. Harvard University Press.

Spijkerboer, Thomas, 2017: Bifurcation of People, Bifurcation of Law, in: Journal of Refugee Studies, 31, 2, S. 216-239.

The Migrants' Files, 2016: The Human and Financial Cost of 15 Years of Fortress Europe, unter: http://www.themigrantsfiles.com [Zuletzt aufgerufen am 18.11.2018].

Toğral, Burcu, 2011: Convergence of Securitization of Migration and 'New Racism' in Europe: Rise of Culturalism and Disappearance of Politics, in: Lazaridis, G. (Hg.), Security, Insecurity and Migration in Europe, S. 220-237. Routledge.

Torpey, John, 1998: Coming and Going: On the State Monopolization of the 'Legitimate Means of Movement', in: Sociological Theory, 16, 3, S. 239-259.

Tsoukala, Anastassia, 2005: Looking at Migrants as Enemies, in: Bigo, D./Guild, E. (Hg.), Controlling Frontiers. Free Movement Into and Within Europe, S. 161-192. Routledge. 
UNITED for Intercultural Action, 2017: List of 33.305 Documented Deaths of Refugees and Migrants Due to the Restrictive Policies of Fortress Europe, unter: http://www.unitedagainstracism.org/wp-content/uploads/ 2017/06/UNITEDListOfDeathsActual.pdf [Zuletzt aufgerufen am 18.11.2018].

van Houtum, Henk, 2010: Human Blacklisting: The Global Apartheid of the EU's External Border Regime, in: Environment and Planning D: Society and Space, 28, 6, S. 957-976.

van Munster, Rens, 2009: Securitizing Immigration. The Politics of Risk in the EU. Palgrave Macmillan.

Zolberg, Aristide R., 2006: A Nation by Design. Immigration Policy in the Fashioning of America. Harvard University Press. 


\section{Annex I: Auflistung der Drittstaaten auf der iSchengener schwarzen Listeı gemäß Verordnung (EG) 539/2001 in der letzten Änderung durch Verordnung VO (EU) 2017/850 vom 17. Mai 2017}

Die `Schengener schwarze Liste $<$ :

\section{Gemeinsame Liste gemäß Artikel 1 Absatz 1}

1. Staaten: Afghanistan, Ägypten, Algerien, Angola, Äquatorialguinea, Armenien, Aserbaidschan, Äthiopien, Bahrain, Bangladesch, Belarus, Belize, Benin, Bhutan, Birma/Myanmar Bolivien, Botsuana, Burkina Faso, Burundi, Central African Republic, China, Côte d'Ivoire, Demokratische Republik Kongo, Dominikanische Republik, Dschibuti, Ecuador, Eritrea, Fiji, Gabun, Gambia, Ghana, Guinea, Guinea-Bissau, Guyana, Haiti, Indien, Indonesien, Irak, Iran, Jamaika, Jemen, Jordanien, Kambodscha, Kamerun, Kap Verde, Kasachstan, Katar, Kenia, Kirgisistan, Komoren, Kongo, Kuba, Kuwait, Laos, Lesotho, Libanon, Liberia, Libyen, Madagaskar, Malawi, Malediven, Mali, Marokko, Mauretanien, Mongolei, Mosambik, Namibia, Nepal, Niger, Nigeria, Nordkorea, Oman, Pakistan, Papua Neuguinea, Philippinen, Ruanda, Russland, Sambia, São Tomé und Príncipe, Saudi-Arabien, Senegal, Sierra Leone, Simbabwe, Somalia, Sri Lanka, Süd Afrika, Sudan, Südsudan, Suriname, Swasiland, Syrien, Tadschikistan, Tansania, Thailand, Togo, Tschad, Tunesien, Türkei, Turkmenistan, Uganda, Usbekistan, Vietnam, Zentralafrikanische Republik.

2. Gebietskörperschaften, die mindestens von einem Mitgliedstaat nicht als Staat anerkannt werden: Kosovo im Sinne der Resolution 1244 des UNSicherheitsrats vom 10. Juni 1999, Palästinensische Behörde. 


\section{Annex II: Auflistung der Drittstaaten auf der iSchengener weißen Listeı gemäß Verordnung (EG) 539/2001 in der letzten Änderung durch Verordnung VO (EU) 2017/850 vom 17. Mai 2017}

Die `Schengener weiße Liste $:$

Gemeinsame Liste gemäß Artikel 1 Absatz 2

1. Staaten: Albanien, Andorra, Antigua and Barbuda, Argentinien, Australien, Bahamas, Barbados, Bosnien und Herzegowina, Brasilien, Brunei Darussalam, Chile, Costa Rica, Dominica, ehemalige jugoslawische Republik Mazedonien, El Salvador, Georgien, Grenada, Guatemala, Honduras, Israel, Japan, Kanada, Kiribati, Kolumbien, Malaysia, Marshallinseln, Mauritius, Mexiko, Mikronesien, Moldau (Republik), Monaco, Montenegro, Nauru, Neuseeland, Nicaragua, Palau, Panama, Paraguay, Peru, Salomonen, Samoa, San Marino, Serbien (ausgenommen Inhaber serbischer Reisepässe, die von der serbischen Koordinationsdirektion ausgestellt wurden), Seychellen, Singapur, St. Kitts und Nevis, St. Lucia, St. Vincent und die Grenadinen, Südkorea, Timor-Leste, Tonga, Trinidad und Tobago, Tuvalu, Ukraine, Uruguay, Vanuatu, Vatikanstadt, Venezuela, Vereinigte Arabische Emirate.

2. Sonderverwaltungsregionen der Volksrepublik China: SAR Hongkong, SAR Macau.

3. Britische Bürger, die nicht Staatsangehörige des Vereinigten Königreichs Großbritannien und Nordirland im Sinne des Unionsrechts sind: britische Staatsangehörige (Überseegebiete) (British Nationals (Overseas)), Bürger der britischen Überseegebiete (British Overseas Territories Citizens), britische Überseebürger (British Overseas Citizens), Personen unter dem Schutz des Vereinigten Königreichs (British Protected Persons), britische Untertanen (British Subjects).

4. Gebietskörperschaften, die von mindestens einem Mitgliedstaat nicht als Staat anerkannt werden: Taiwan. 



\section{Verpflichtung zur aktiven Seenotrettung \\ Zu den nicht intendierten Effekten der Überwachung des Mittelmeers durch Frontex}

Maximilian Pichl / Timo Tohidipur

\section{EINLEITUNG}

Die Erinnerung an große Schiffskatastrophen gehört zum festen Bestandteil der europäischen (Kultur-)Geschichte. Nicht zuletzt das weltberühmte Gemälde „Das Floß der Méduse“ von Thédore Géricault, das an die Katastrophe der französischen Fregatte Méduse von 1816 erinnert und heute im Pariser Louvre zu sehen ist, ist für dieses Gedenken kennzeichnend. Neben der Méduse haben auch die Schiffskatastrophen der Titanic, der Lusitana, der Wilhelm-Gustloff oder die Costa Concordia Eingang in das kollektive Gedächtnis der europäischen Gesellschaften gefunden.

Umso erstaunlicher ist es, dass sich in den letzten Jahren in Teilen der europäischen Öffentlichkeit eine gewisse Normalisierung angesichts des alltäglichen Sterbens von Geflüchteten im Mittelmeer beobachten lässt. Nachdem die Europäische Union im Jahr 2001 mit der sog. Carrier Sanctions Richtlinie ${ }^{1}$ es Transportunternehmen unter der Androhung von Sanktionen untersagte Personen ohne gültige VISA-Papiere zu befördern und die EU-Mitgliedstaaten gleichzeitig ihre Einwanderungskontrollen verschärften, wurden legale und sichere Zugangswege nach Europa für Menschen auf der Flucht faktisch verschlossen. Die Fluchtbewegungen verlagerten

1 Richtlinie 2001/51/EG vom 28. Juni 2001. 
sich auf das Mittelmeer und wurden für die Betroffenen länger und gefährlicher. Gerade in den vergangenen vier Jahren ist die Europäische Grenze zur tödlichsten Grenze der Welt geworden. Nach Schätzungen der International Organisation for Migration (IOM) starben alleine im Zeitraum zwischen 2014 und 2017 ca. 14.500 Menschen im Mittelmeer ${ }^{2}$, wobei viele Vorfälle nicht dokumentiert werden können. Während das Sterben auf dem Mittelmeer viele zivilgesellschaftliche Initiativen bewegt hat aktiv zu werden und die Seenotrettung selbst in die Hand zu nehmen, wofür Organisationen wie SeaWatch, Jugendrettet oder Seaeye stellvertretend stehen, hat die EU bislang auf die Einrichtung einer eigenen EU-Küstenwache verzichtet. Stattdessen wurde die bereits bestehende Überwachung und Absicherung der EU-Außengrenze intensiviert. Die Europäische Grenzagentur Frontex wurde 2016 zur Europäischen Agentur für die Grenz- und Küstenwache weiterentwickelt und erhält immer stärker das Gepräge einer eigenen europäischen Grenzpolizei. Von Ende 2014 bis 2018 koordinierte Frontex die Operation Triton vor der italienischen Küste, die die Seenotrettungsoperation Mare Nostrum ersetzte und stattdessen das Ziel einer Sicherung der Außengrenzen verfolgte, wie der Generaldirektor von Frontex, Gil Arias, in einem Gespräch selbst einräumte. ${ }^{3}$ Die im Februar 2018 neu initiierte Frontex Operation Themis, die Triton ersetzt, festigt diese Tendenz und setzt zudem einen verstärkten Fokus auf Kriminalitätsbekämpfung. Die Operationsweise von Frontex hat daher ihren eindeutigen Schwerpunkt in der Grenzkontrolle, die Funktion der Küstenwache übernimmt Frontex eher zufällig. Diese grenzpolizeiliche Frontex-Mission wird zudem ergänzt durch die militärische Operation EUNAFVOR MED/Sophia, deren Hauptziel die Bekämpfung des Menschenschmuggels zwischen Libyen, Tunesien und Italien ist. Obschon sowohl Schiffe von Frontex wie auch der Operation Sophia an der Rettung von in Seenot geratenen Flüchtlingen beteiligt

2 Internation Organisation of Migration (2017) Fatal Journeys. Volume 3 Part 1. Improving Data on Missing Migrants, Genf, S. 6; Eine aktualisierte Übersicht über die Todeszahlen im Mittelmeer findet sich auf der Website Missing Migrant: https://missingmigrants.iom.int/region/mediterranean (letzter Aufruf am 18.01.2019).

3 http://www.europaforum.public.lu/fr/actualites/2014/09/pe-comm-libe-frontextriton/index.html_(letzter Aufruf am 18.01.2019). 
sind, geht mit ihren Einsätzen auch eine stärkere Militarisierung der Grenze einher. ${ }^{4}$

In der Agentur Frontex verbindet sich eine Dialektik von Grenzabschottung und Seenotrettung, die zu nichtintendierten Effekten führt. Um ihrem Zweck der Grenzkontrolle zu genügen, ist die Agentur auf vielfältige Überwachungstechnologien angewiesen. Zugleich ist die Agentur in einen europa- und seerechtlichen Rahmen eingebettet, der mit verschiedenen Verpflichtungen in Bezug auf die Seenotrettung einhergeht. In diesem Beitrag soll der Gedanke vorgestellt werden, dass sich gerade aus der intensiven Überwachung des Mittelmeers durch Frontex eine pro-aktive Verpflichtung zur Rettung von in Seenot geratenen Geflüchteten ergibt, der sukzessive die Grenzkontrollfunktion der Agentur zugunsten ihrer Funktion als Küstenwache überwölben dürfte. Freilich bleibt die grundsätzliche Kritik an Frontex als Operatorin einer exekutiven Sicherheitslogik in der Migrationspolitik virulent.

Zunächst werden die wesentlichen rechtlichen und politischen Auseinandersetzungen über die Seenotrettung im Mittelmeer seit Anfang der 2000er Jahre bis zum Jahr 2018 rekonstruiert. ${ }^{5}$ Anschließend wird die Struktur und der Auftrag von Frontex sowie ihre Bindung an seerechtliche und menschenrechtliche Vorgaben skizziert. Darauf aufbauend wird gezeigt, dass gerade die spezifischen Überwachungsressourcen der Agentur im Kontext der Migrationsrouten über das Mittelmeer nicht-intendierte Effekte bewirken und eine proaktive Verpflichtung zur Seenotrettung auslösen.

4 Thomas Schmid, „Aus den Augen, aus dem Sinn“ - Flüchtlinge und Migranten an den Rändern Europas. Die zentrale Mittelmeerroute, Heinrich-Böll-Stiftung, Berlin 2016, S. 21.

5 Die 2018 federführend durch den extrem rechten italienischen Innenminister Matteo Salvini (Lega-Partei) begonnene, inhumane Schließung (süd-)europäischer Häfen für Schiffe mit Schutzsuchenden an Bord, die im Falle der italienischen Häfen sogar zur Abweisung von Schiffen der eigenen Küstenwache und von Frontex führte, kann hier zwar aus Zeitgründen nicht mehr umfassend dokumentiert werden, bestärkt uns aber nur in unserer Analyse. 


\section{RECHTLICHE UND HISTORISCHE AUSEINANDERSETZUNGEN UM DIE SEENOTRETTUNG}

Die Verpflichtung zur Seenotrettung ist in verschiedenen völkerrechtlichen Verträgen kodifiziert: im Seerechtsübereinkommen der Vereinten Nationen (SRÜ), dem Internationalen Übereinkommen zum Schutz menschlichen Lebens auf See und im Internationalen Abkommen über Seenotrettung. Im SRÜ, der „Verfassung der Meere“" ${ }^{6}$, heißt es in Art. 98 Abs. 1: „Jeder Staat verpflichtet den Kapitän eines seine Flagge führenden Schiffes, soweit der Kapitän ohne ernste Gefährdung des Schiffes, der Besatzung oder der Fahrgäste dazu imstande ist, jeder Person, die auf See in Lebensgefahr angetroffen wird, Hilfe zu leisten“ sowie ,,so schnell wie möglich Personen in Seenot zu Hilfe zu eilen, wenn er von ihrem Hilfsbedürfnis Kenntnis erhält, soweit diese Handlung vernünftigerweise von ihm erwartet werden kann.“ Die Verpflichtung zur Seenotrettung hat also eine doppelte Bedeutung: Sie verpflichtet Schiffe zur Rettung, wenn sie zufällig in Seenot befindlichen Personen begegnen, aber auch zur proaktiven Rettung, wenn sie Kenntnisse über den Ort und Zustand der Personen haben. Zur Seenotrettung sind alle Schiffe verpflichtet, nicht nur staatliche. Tatsächlich sind es in der Praxis oft private Handelsschiffe, die zur Seenotrettung eingesetzt werden. Das Recht der Seenotrettung entwickelte sich vor allem aus der maritimen Tradition und wurde später in den völkerrechtlichen Verträgen kodifiziert, ${ }^{7}$ ist mithin als geltendes Völkergewohnheitsrecht anzusehen. ${ }^{8}$

Die See ist durch das SRÜ zudem auf eine spezifische rechtliche Art und Weise aufgeteilt, die sich auch auf die Seenotrettung auswirkt. Im Wesentlichen sind dabei vier Zonen von Relevanz: Innerhalb des Küstenmeers, das bis zu zwölf Seemeilen vom Festland aus reicht, übt der zuständige Staat volle Hoheitsbefugnisse aus. In der darauffolgenden Anschluss-

6 Sicco Rah, Asylsuchende und Migranten auf See. Staatliche Rechte und Pflichten aus völkerrechtlicher Sicht, Hamburg Studies in Maritime Affairs 15, Berlin/Heidelberg 2009, S. 15.

7 Ebd., S. 100.

8 Wissenschaftlicher Dienst des Bundestages, Die völkerrechtliche Pflicht zur Seenotrettung, WD 2 - 3000-053/17, S. 5 mit weiteren Nachweisen. 
zone, die ebenfalls zwölf Seemeilen umfasst, hat der Staat die Befugnis die Zoll- und Einreisebestimmungen zu kontrollieren. Die sich bis auf 200 Seemeilen erstreckende ausschließliche Wirtschaftszone reguliert die Nutzung von Bodenschätzen. Schließlich unterliegt die anschließende Hohe See keiner alleinigen staatlichen Souveränität, sondern wird als allgemeines Erbe der Menschheit eingestuft (Common Heritage of Mankind). Ereignet sich eine Schiffskatastrophe auf der Hohen See schließt dies nun gerade nicht aus, dass ein in der Nähe befindliches Schiff aufgrund fehlender staatlicher Souveränitätsrechte nicht eingreift. Die Verpflichtung zur Seenotrettung erstreckt sich auf alle Gebiete der See. Sie kann unter Umständen auch Schiffe dazu zwingen in die Hoheitsgewässer eines fremden Staates zu fahren, sofern dortige Schiffe nicht gleich effektiv zur Seenotrettung beitragen können.

In der Praxis der Seenotrettung, und dies ist gerade im Kontext der Fluchtbewegungen über das Mittelmeer von höchster Relevanz, ist es umstritten, wohin die aus Seenot Geretteten verbracht werden sollen. Die MSC Guidelines on the treatment of persons rescued at sea sind unverbindliche Regeln, die von der Internationalen Seeschifffahrts-Organisation verabschiedet wurden, um Kapitänen Handlungsempfehlungen für die Seenotrettung zur Verfügung zu stellen. Der International Convention on Maritime Search and Rescue (SAR) zufolge sind Schiffbrüchige an einen ,sicheren Ort“ zu bringen (Annex, $\S$ 1.3.2). Die MSC Guidelines definieren den „,sicheren Ort“ gemäß Punkt 6.12 wie folgt: „A place of safety [...] is a location where rescue operations are considered to terminate. It is also a place where the survivors' safety of life is no longer threatened and where there basic human needs (such as food, shelter and medical needs) can be met." Der Richtlinie zufolge soll der "sichere Ort" also anhand des konkreten Einzelfalls definiert werden, wodurch den Kapitänen die nicht leichte Aufgabe zukommt ein gewisses Ermessen auszuüben. Es kommt darauf an welcher Ort für die konkret betroffenen Personen einen Schutz und ausreichende Versorgung anbietet, weshalb nicht unbedingt der nächstgelegene Hafen auch ein ,sicherer Hafen“ sein muss. Im Hinblick auf die Situation von Flüchtlingen wird das Seerecht zudem durch flüchtlings- und menschenrechtliche Aspekte überlagert. Ein ,sicherer Ort“ für Flüchtlinge bemisst sich an den spezifischen Bedürfnissen dieser Gruppe. Der Europäi- 
sche Gerichtshof für Menschenrechte (EGMR) hat in seinem wegweisenden Urteil in der Rechtssache Hirsi Jamaa u.a. gegen Italien ${ }^{9}$ dargelegt, welche Kriterien bei der Seenotrettung von Flüchtlingen zu beachten sind. In dem konkreten Fall hatten italienische Grenzschützer ein in Seenot geratenes Boot mit Flüchtlingen aus Somalia und Eritrea abgefangen und die Personen nach Libyen gebracht, ohne ihnen die Möglichkeit zu geben in einem individuellen Verfahren ihre Schutzgründe auf italienischem Territorium zu prüfen. Dem EGMR zufolge verstieß diese Push-Back-Operation gegen Art. 3, 13 Europäische Menschenrechtskonvention (EMRK) sowie gegen das Verbot der Kollektivausweisung aus dem Art. 4 des 4. Zusatzprotokolls zur EMRK. Der EGMR bekräftigte, dass bei der Seenotrettung von Flüchtlingen das sog. non-refoulement-Gebot zu beachten sei, demzufolge Flüchtlinge nicht in Staaten gebracht werden dürfen, in denen ihnen Verfolgung, Folter oder eine unmenschliche Behandlung droht. ${ }^{10}$

Obwohl die internationalen Rechtsregeln eindeutige Pflichten zur Seenotrettung vorsehen, kam es im Kontext der Fluchtbewegungen von Flüchtlingen nach Europa immer wieder zu dramatischen Fällen, in denen die Retter*innen durch die EU-Mitgliedstaaten kriminalisiert wurden. Am 20. Juni 2004 rettete das unter deutscher Flagge fahrende Schiff Cap Anamur südlich von Lampedusa 37 Menschen das Leben, die in Libyen in See gestochen waren. Als die Crew den Hafen von Porto Empedocle auf Sizilien erreichte, wurde das Schiff zwölf Tage lang mit militärischen Mitteln am Einlaufen gehindert. Der damalige deutsche Innenminister Otto Schily (SPD) spekulierte in der Presse offen darüber, dass sich die Besatzung der Cap Anamur an einer strafrechtlich erheblichen Schleusung beteiligt habe und lehnte eine Aufnahme der Flüchtlinge in Deutschland ab. ${ }^{11}$ Schon damals forderte Innenminister Schily die Errichtung von Flüchtlingslagern außerhalb Europas - ein Vorschlag der im Kontext der Krise der europäischen Migrationspolitik seit 2015 von vielen Akteuren fälschlicherweise als innovative Idee präsentiert wurde. Gegen den Kapitän und den

9 EGMR HIRSI JAMAA AND OTHERS V. ITALY, Application no. 27765/09 v. 23. Februar 2012.

10 Der EGMR entnimmt dieses ursprünglich aus Art. 33 Genfer Flüchtlingskonvention stammende Prinzip auch aus Art. 3 EMRK.

11 Spiegel Online vom 17. Juli 2004. 
Vorsitzenden von Cap Anamur wurden in Italien Strafverfahren wegen Beihilfe zur illegalen Einreise eingeleitet, die erst im Jahre 2009 mit einem Freispruch endeten. Ein ähnliches Schicksal wie die Besatzung der Cap Anamur erfuhren auch tunesische Fischer, die im Jahr 2007 in Seenot geratene Flüchtlinge nach Lampedusa brachten. Auch sie wurden zunächst angeklagt, ihr Verfahren später eingestellt. Trotz der Freisprüche hatten die Verfahren fatale Folgen, weil sie die Seenotrettung per se in Verbindung mit Straftaten brachten und dazu beitrugen, andere Schiffscrews von ihrer Verpflichtung zur Seenotrettung abzuschrecken.

Erst durch das Hirsi-Urteil des EGMR änderte sich die Situation auf dem Mittelmeer. Italien sah sich nun dazu gezwungen aus Seenot Gerettete aufs Festland zu bringen und dort ihre Asylverfahren durchzuführen. Der italienische Staat ergriff aber auch proaktiv die Initiative und startete die Mission Mare Nostrum, die innerhalb des Jahres 2014 ca. 150.000 Menschen aus Seenot rettete. Die italienischen Schiffe operierten dabei bis in die libyschen Gewässer hinein, um mehr Menschen das Leben zu retten. Aufgrund der großen humanitären Reichweite der Operation geriet sie ins Visier der Kritik. Wurden zuvor vor allem private Seenotretter*innen angegriffen, so musste sich nun Italien für seine Politik rechtfertigen. Der deutsche Innenminister Thomas de Maizière (CDU) griff Mare Nostrum offensiv an, indem er davon sprach, die Operation sei als Nothilfe gedacht gewesen und habe sich als Brücke nach Europa erwiesen ${ }^{12}$ - er brachte auf diese Weise die Seenotrettung mit dem Geschäft von Schleusern unmittelbar in Verbindung, offensichtlich um ihre öffentliche Legitimität zu untergraben. Mare Nostrum wurde eingestellt und durch die Frontex-Operation Triton ersetzt, die weniger finanzielle Mittel erhielt, einen kleineren Operationsradius hatte und explizit nicht als Seenotrettungsmission ausgewiesen wurde. ${ }^{13}$ Die nun seit Februar 2018 darauf folgende Frontex Operation Themis vertieft diese Entwicklung, wie bereits ausgeführt, deutlich.

Im Kontext der gestiegenen Fluchtbewegungen über das Mittelmeer im Laufe des Jahres 2015 wurden verstärkt private NGOs aktiv, die sich an der

12 http://www.deutschlandfunk.de/fluechtlinge-italien-beendet-rettungsaktion-mare -nostrum.1818.de.html?dram:article_id=301920 (letzter Aufruf am 18.01.2019).

13 https://www.proasyl.de/news/europas-schande-triton-und-mare-nostrum-im-ver gleich/_(letzter Aufruf am 18.01.2019). 
Seenotrettung beteiligten. Dabei traten so unterschiedliche Akteure auf wie die Migrant Offshore Aid Station (MOAS), Ärzte ohne Grenzen, Sea Watch, Jugend Rettet oder Sea Eye. Einige der Akteure verstanden ihre Aufgabe der Seenotrettung auch als politische Positionierung, indem sie sich offensiv für legale Zugangswege für Flüchtlinge nach Europa einsetzten. ${ }^{14}$ Die NGOs schafften es zum Teil, dass die black-box Mittelmeer eine größere Öffentlichkeit erhielt und die unterlassene Rettung von Flüchtlingen stark politisiert wurde. Besonders die Rolle von SeaWatch ist in diesem Zusammenhang bemerkenswert:

„Zum einen fordert die Organisation Europäische EntscheidungsträgerInnen durch ihre praktischen Interventionen heraus, indem sie auf dem Mittelmeer Rettungen übernimmt, gleichzeitig aber darauf hinweist, dass sie sich selbst nur als zweitbesten Akteur für diese Aufgabe sieht und ihr Eingreifen nur kurzfristig das Sterben auf See reduzieren soll. Zum anderen interveniert sie diskursiv, indem sie die Öffentlichkeit informiert, das staatliche Informationsmonopol über die Geschehnisse vor Ort aufbricht und alternative Darstellungen bereitstellt. Dadurch kratzt Sea Watch am humanitären Anstrich der nationalstaatlichen und EU-Missionen im Mittelmeer, die zum Ziel haben, MigrantInnen vor kriminellen SchleuserInnen zu schützen, dabei aber den unmittelbaren Schutz des Lebens durch Seenotrettungen zu vernachlässigen scheinen. ${ }^{\text {"15 }}$

Ein Teil der privaten NGOs arbeitete auch mit der italienischen Küstenwache und sogar partiell mit den Akteuren der militärischen Operationen zusammen. Ihre Operationen reichten dabei von Erkundungsmissionen nach in Seenot geratenen Schiffen, über die Mitteilung der jeweiligen Standorte an die italienische Küstenwache bis zur sofortigen Hilfe und Übernahme der Betroffenen auf ihre Boote. Im Verlauf des Jahres 2017

14 Paolo Cuttitta, Zwischen De- und Repolitisierung. Nichtstaatliche Search and Rescue-Akteure an der EU-Mittelmeergrenze. In: Hess, Sabine u.a. (Hrsg.) Der lange Sommer der Migration. Grenzregime III, Berlin/Hamburg 2017, S. 115 125, hier S. 118.

15 Linda Ewert, „Suchen. Retten. Legalisieren.“ Chancen und Grenzen zivilgesellschaftlicher Seenotrettung am Beispiel der Organisation Sea Watch. In: Hess, Sabine u.a. (Hrsg.) Der lange Sommer der Migration. Grenzregime III, Berlin/Hamburg 2017, S. 126 - 136, hier S. 133. 
gerieten die NGOs bei ihrer Arbeit immer stärker ins Visier der europäischen Behörden. Die EU hatte zuvor intensiv mit den nordafrikanischen Staaten, darunter auch Libyen, verhandelt, mit dem Ziel, erneut den Grenzschutz an Drittstaaten außerhalb Europas auszulagern. Speziell die Zusammenarbeit mit Libyen evozierte öffentliche Kritik, weil der Staat seit dem Arabischen Frühling politisch fragmentiert ist, die Zustände in den dortigen Lagern menschenunwürdig sind und auch der libyschen Küstenwache schwere Menschenrechtsverletzungen vorgeworfen werden. Sea Watch berichtete zum Beispiel von einem Vorfall bei dem die libysche Küstenwache die NGO dabei behinderte Menschen aus Seenot zu retten, was zum Tod einiger Insassen geführt haben soll. ${ }^{16}$ Der Fall wird aktuell vor dem EGMR verhandelt.

Die Arbeit der Seenotrettungsorganisationen wurde ab dem Sommer 2017 massiv beschränkt, erneut unter der Anführung des deutschen Innenminister Thomas de Maizière. Die europäischen Innenminister einigten sich auf einen sog. Verhaltenskodex für NGOs, den die italienischen Behörden umsetzen sollten, um die Arbeit der Organisationen schärfer zu kontrollieren. Zu dem Kodex gehört das Verbot für private Seenotrettungsorganisationen in libysche Gewässer zu fahren. Gerettete Personen sollen zudem nicht wie üblich auf andere Schiffe umsteigen, sondern sofort in einen sicheren Hafen verbracht werden. Aus der Sicht von Sea Watch würde dies dazu führen, dass weniger Schiffe auf dem Meer wären, um potentielle Seenotopfer zu finden. Außerdem sieht der Kodex vor, dass Polizeibeamte an Bord der Schiffe sein sollen, um gegen eventuelle Schleuser zu ermitteln. Unterschreiben die Organisationen den Kodex nicht, so sollen sie keine Einfahrterlaubnis in italienische Häfen erhalten. Das Schiff Iuventa der Organisation Jugend Rettet wurde zum Beispiel beschlagnahmt, weil man der Crew, ohne belebgare Nachweise anzuführen, die Beihilfe zur illegalen Einreise vorgeworfen hatte. Jugend Rettet hatte sich geweigert den Kodex zu unterzeichnen (nach aktuellen Stand ist die Iuventa weiterhin beschlagnahmt, Oktober 2018). Einer umfangreichen Recherche der ZEIT zufolge laufen die Vorwürfe gegen die Organisationen ins Leere: „Private Rettungsschiffe im Mittelmeer helfen Schleuserbanden, behaupten EUPolitiker. Doch eine Auswertung der Positionsdaten zeigt: Die Helfer halten

16 Süddeutsche Zeitung 2. Januar 2018. 
sich an die Regeln." Sie zitieren dabei aus einer Studie der Universität Oxford: „Verstärkte Rettungsaktionen führen keineswegs zu vermehrter Flucht. Der Vorwurf, mehr Retter produzierten mehr Flüchtlinge, ist demnach falsch. Ebenso die Formel, dass mehr Rettungsboote mehr Flüchtlinge anlocken würden. Den einzigen Zusammenhang, den Steinhilper und Gruijters fanden: Es gibt weniger Tote, wenn mehr Retter auf dem Meer unterwegs sind." ${ }^{\text {"17 }}$

Neben den nicht belegbaren Vorwürfen wirft der Verhaltenskodex auch zahlreiche rechtliche Fragen hinsichtlich seiner völkerrechtlichen Vereinbarkeit auf. Dabei wird bereits in Abrede gestellt, dass der Verhaltenskodex überhaupt Sanktionen durchsetzen könnte, weil er eher eine Leitlinie statt eine verbindliche Vorgabe darstelle. ${ }^{18} \mathrm{Zu}$ diesem Ergebnis kommt auch ein Gutachten des Wissenschaftlichen Dienst des Deutschen Bundestags, dass darüber hinaus klarstellt: „Vor diesem Hintergrund dürfen private Schiffe, die etwa auf der Reise zwischen zwei Ländern sind, das Küstengewässer eines dritten Landes ohne besondere Genehmigung benutzen. Nach Art. 18 Abs. 2 S. 2 SRÜ i.V.m. Art. 98 SRÜ haben sie bei der Durchfahrt der Küstengewässer sogar die Pflicht, fremden Schiffen oder Personen in Seenot Hilfe zu leisten. Diese Pflicht bestünde auch, wenn das private Schiff noch außerhalb des Küstenmeeres fährt und auf ein Schiff aufmerksam wird, welches in den Küstengewässern in Seenot geraten ist. “" ${ }^{19}$ Der Verhaltenskodex für private NGOs entfaltet vor diesem Hintergrund weder eine Sanktionsmöglichkeit noch steht er im Einklang mit den seerechtlichen und völkerrechtlichen Vorgaben.

Der kurze historische Abriss zeigt drei Entwicklungen auf: Erstens ist die Seenotrettung von Geflüchteten, trotz eindeutiger seerechtlicher Vorgaben, politisch bis heute höchst umstritten. Zweitens versuchen politische Verantwortliche die Seenotrettung einerseits diskursiv zu delegitimieren, indem sie in Zusammenhang mit Kriminalität gestellt wird, und andererseits werden rechtliche Strategien verfolgt, um die klaren seerechtlichen

17 Zeit Online, Weniger Helfer bedeuten mehr Tote, 17. Juli 2017.

18 Lena Zagst, Bedenklicher italienischer Verhaltenskodex für Seenotrettung durch NGOs im Mittelmeer, Juwiss-Blog vom 15. Dezember 2017, https://www. juwiss.de/139-2017/_(letzter Aufruf am 18.01.2019).

19 WD $2-3000-068 / 17$. 
Vorgaben zu umgehen. Drittens hat der Einsatz von staatlichen Akteuren, darunter Frontex, seit 2015 nicht dazu beigetragen, das Sterben auf dem Mittelmeer zu beenden; abgesehen von der italienischen Mission Mare Nostrum zwischen 2013 bis 2014 ist eher eine Tendenz zu beobachten, derzufolge die Delegitimierung des zivilgesellschaftlichen Engagements beim gleichzeitigen Einsatz von vornehmlich grenzpolizeilich ausgerichteten Akteuren zu mehr Toten auf dem Mittelmeer führt.

\section{EINFÜHRUNG EINES INTEGRIERTEN GRENZSCHUTZSYSTEMS}

Wie in der historischen Rekonstruktion der Europäischen Seenotrettung deutlich wurde, übernimmt Frontex eine immer stärkere Rolle aufgrund der Operationen im Mittelmeer, während gleichsam die Aktivitäten privater NGOs unter Beschuss sind. In der EU verdichtet sich die Wechselwirkung zwischen Grenzschutz und Seenotrettung paradigmatisch in der Agentur Frontex und ihrem Zusammenspiel mit den mitgliedstaatlichen Grenzschutzbehörden und der Kooperation mit Drittstaaten. Die Agentur agiert auf ihrer 2016 neu gefassten Rechtsgrundlage, einer Verordnung der Europäischen Union $\left(\right.$ VO 2016/1624 = Frontex-VO) ${ }^{20}$, die im ordentlichen Rechtsetzungsverfahren beschlossen wurde und die Agentur in ihrem Mandat und operativen Befugnissen im Vergleich zu früher stärkt. Schon die Umbenennung vom früheren, eher sperrigen Namen „Europäischen Agentur für die operative Zusammenarbeit an den Außengrenzen der Mitgliedstaaten der Europäischen Union“, wo die eigene Rolle hinter der der Mitgliedstaaten schon im Namen scheinbar zurücksteht, zur „Europäischen Agentur für die Grenz- und Küstenwache“ verdeutlicht die Zuschreibung einer selbstbewussteren und erweiterten Rolle für die Agentur im „Management" der EU-Außengrenze. Frontex emanzipiert sich fortwährend im Einklang mit der ihr politisch zugestandenen Rolle und gestaltet die der Agentur eingeräumten Entscheidungsspielräume in Eigenregie weiter aus.

20 Verordnung (EU) 2016/1624 des Europäischen Parlaments und des Rates vom 14. September 2016 über die Europäische Grenz- und Küstenwache. 
Frontex ist als Agentur geschaffen worden, um die Wirksamkeit der integrierten europäischen Grenzverwaltung in der Praxis zu gewährleisten (Erwägungsgrund 5 der Frontex-VO 2016). Die Kompetenzen der Europäischen Union bezüglich der Grenzkontrolle basieren maßgeblich auf Art. 77 und 79 des Vertrages über die Arbeitsweise der Europäischen Union (AEUV). Dieser Kompetenzrahmen umfasst die gemeinsame Visa-Politik, Maßnahmen zur Kontrolle an den Außengrenzen der EU bei gleichzeitiger Abschaffung der Binnenkontrollen, Aufenthaltsbedingungen von Drittstaatsangehörigen sowie gemeinsame Maßnahmen zur Abschiebung und Rückführung und vor allem die Einführung eines integrierten Grenzschutzsystems an den Außengrenzen. Die Entwicklung einer Politik der EU zur wirksamen Ausgestaltung dieses integrierten Grenzschutzsystems an den Außengrenzen kann dabei ausdrücklich alle Maßnahmen umfassen, die hierzu erforderlich sind. Als ,erforderliche“ Maßnahmen wurden materiell der Schengener Grenzkodex und der Visakodex verabschiedet, die die gesamten Modalitäten von Grenzkontrollen, Grenzübertritt und Visagewährung festlegen. ${ }^{21}$ Und als institutionell erforderlich wurde aus der Sicht der EU die Etablierung einer eigenen Behörde, die Agentur Frontex, angesehen.

Die Kompetenzverteilung zwischen der EU-Agentur und den Grenzbehörden der EU-Mitgliedstaaten ist durchaus schwierig und jedenfalls nicht streng hierarchisch organisiert, denn während rechtlich grundsätzlich ein Vorrang des EU-Rechts gilt, ${ }^{22}$ gehört die Entscheidung darüber, wer eine staatliche Grenze überschreiten darf, zu den hoheitsrechtlichen Kompetenzen, die sich Staaten nur sehr ungerne nehmen lassen und auch nicht einfach auf andere Einrichtungen übertragen. Selbst für das vom Europarecht überformte Flüchtlingsrecht gilt grundsätzlich immer noch: „Protection is State Protection“. ${ }^{23}$ Mit der Koordinierung des Grenzmanagements an den

21 Mehr dazu im Beitrag von Thea Kirsch in diesem Sammelband.

22 Thomas Oppermann/Claus Dieter Classen/Martin Nettesheim, Europarecht, 8. Aufl., München 2018, S. 152 ff.

23 James Hathaway/Michelle Foster, The Law of Refugee Status, 2nd ed 2014, 289 und 292; vgl. weiter hierzu: What is the Meaning of State Protection in Refugee Law? A Debate James C Hathaway, Hugo Storey, International Journal of Refugee Law, Volume 28, Issue 3, 1 October 2016, S. 480-492. 
Außengrenzen der EU und damit den Außengrenzen der EU-Mitgliedstaaten, knüpft die Tätigkeit von Frontex allerdings genau an diesen staatlichen Hoheitsbefugnissen an. Frontex versteht sich als „operativer Arm“ der EU und in dieser Funktion als Partner der Mitgliedstaaten mit Blick auf Außengrenzkontrollen. ${ }^{24}$

Die neue Frontex-Verordnung spricht hier von ,gemeinsamer Verantwortung“, d.h. verantwortlich sind die Agentur Frontex und die für die Grenzverwaltung und Küstenwache zuständigen nationalen Behörden. Dies inkludiert eine Vielzahl unterschiedlicher Behörden. In Deutschland beispielsweise gehört zur Küstenwache neben dem maritimen Arm der Bundespolizei, die ihrerseits auch Grenzpolizei ist, weitere Vollzugskräfte des Bundes zur See, wie der Zoll, die Wasser- und Schifffahrtsverwaltung und der Fischereischutz, die in einem gemeinsamen Lagezentrum zusammenarbeiten. $^{25}$

Es besteht also einmal eine Verantwortung der Mitgliedstaaten für den Schutz ihrer Abschnitte der Außengrenzen - hierfür haben sie die ,vorrangige Zuständigkeit“. Doch die Kontrolle der EU-Außengrenzen in ihrer Gesamtheit ist eine Gesamtverantwortung aller Mitgliedstaaten sowie der EU und fällt somit in den Managementbereich von Frontex. Die Agentur ist in diesem Zusammenhang befugt sogenannte „Gefährdungsbeurteilungen“ zu erstellen und so „Schwachstellen“ an den Außengrenzen zu ermitteln, auf die dann z.B. durch Frontex-Einsätze reagiert werden kann.

Die Agentur übernimmt folglich eine strategische Organisationsverantwortung, denn nur wenn alle Informationen, die den Mitgliedstaaten über die Situationen an und jenseits der Außengrenzen auch Frontex vorliegen, ist aus Sicht einer Grenzschutzbehörde effektives strategisches Handeln möglich. Dies bedeutet letztlich eine informationelle Zentralisierung. Ähnliche Tendenzen stärkerer Zentralisierung zeigen sich auch dadurch, dass Frontex in dringenden Fällen durch einen Durchführungsrechtsakt von Rat und Kommission auch ohne mitgliedstaatliche Anfrage eingesetzt werden

24 Frontex Risk Analysis 2018, S. 6.

25 Die Aufgaben der Suche und Rettung auf See in Nord- und Ostsee wurden indes der privaten Deutschen Gesellschaft zur Rettung Schiffbrüchiger (DGzRS) übertragen, vgl. Wissenschaftlicher Dienst des Bundestages, WD 5 - 3000 - 124/18, 2018, S. 7. 
kann und der Mitgliedstaat, in dem der Einsatz stattfinden soll, dem EUBeschluss und damit dem Soforteinsatz von Frontex Folge leisten muss (Art. 19 Frontex-VO). Der Mitgliedstaat kann dann nicht mehr über das „ob“, sondern nur noch über das „wie“ des Einsatzes mitbestimmen, und zwar durch Mitarbeit am Frontex-Einsatzplan.

Dass dies nur ,in dringenden Fällen“ möglich sein wird zeigt indes, dass es sich hier um eine Sondersituation handelt und nicht um den täglichen Normalfall. Zudem ist durch die zuvorige Entscheidung von Kommission und Rat aus Sicht der Mitgliedstaaten abgesichert, dass Frontex nicht eigenmächtig über Einsätze an den Grenzen von Mitgliedstaaten entscheiden darf. Gleichwohl ist schon die Tatsache, dass ein Frontex Einsatz auch ohne mitgliedstaatliche Anfrage beschlossen werden kann und der Mitgliedstaat Folge leisten muss, ein nicht zu unterschätzender weiterer Schritt Richtung Zentralisierung des EU-Außengrenzschutzes. Hier verdeutlichen sich in einer langfristigen Perspektive die Grundmuster einer originären Europäischen Grenzpolizei, die eigenständig neben oder gar statt der mitgliedstaatlichen Grenzbehörden handelt. Dies wird so durchaus auch in der EU im Rahmen der politischen Agenda verhandelt. So sprach z.B. der französische Staatspräsident Emmanuel Macron in der nach seinem Amtsantritt recht vielbeachteten Europarede 2017 ausdrücklich von der Schaffung einer „europäischen Grenzpolizei“ ${ }^{26}$ und tendenziell vergleichbar, wenngleich etwas weniger deutlich, sprach der Präsident der Europäischen Kommission Jean-Claude Juncker in seiner Rede zur Lage der Union 2017 davon, dass „gemeinsame Grenzen und gemeinsamer Grenzschutz“ zusammen gehören. ${ }^{27}$ Dieser jetzt schon präsente und stetig anwachsende Bedeutungszuwachs für Frontex als Agentur führt aber zugleich zu einem entsprechenden Zuwachs an rechtlicher Verantwortlichkeit.

26 Rede von Staatspräsident Macron an der Sorbonne - Initiative für Europa, Paris 26. September 2017, S. 5, https://de.ambafrance.org/Initiative-fur-Europa-DieRede-von-Staatsprasident-Macron-im-Wortlaut (letzter Aufruf am 18.01.2019).

27 Präsident Jean-Claude Juncker, Rede zur Lage der Union 2017, Brüssel, 13. September 2017, vgl. http://europa.eu/rapid/press-release_SPEECH-17-3165_ de.htm (letzter Aufruf am 18.01.2019). 


\section{AMBIVALENZEN IM AUFTRAG VON FRONTEX}

Eine deutliche Ambivalenz zwischen einem Sicherheitsauftrag, der im Kern auf grenzbezogene Selektionsvorgänge ausgerichtet ist, und der Verpflichtung auf die Einhaltung der Menschenrechte, zieht sich durch alle Aufgabenfelder und Rechtsgrundlagen der Agentur und gehört so zum Konstruktionsdilemma und -defizit von Frontex, das sich nur schwer rechtsstaatlich auflösen lässt. $^{28}$

So umfasst der Handlungsrahmen der integrierten europäischen Grenzverwaltung zunächst Grenzkontrollen und die damit zusammenhängenden Kooperationen zwischen EU und den Behörden der Mitgliedstaaten sowie Drittstaaten. Die Frontex-VO konkretisiert als Zielvorstellung für die Agentur zunächst eine ,effiziente Steuerung des Überschreitens der Außengrenzen und damit die Bewältigung von Migrationsdruck sowie potenziellen künftigen Bedrohungen an diesen Grenzen." Die Agentur soll in diesem Zusammenhang ausdrücklich einen Beitrag zur Bekämpfung von schwerer Kriminalität mit grenzüberschreitender Dimension leisten und dazu beitragen, ein hohes Maß an innerer Sicherheit in der Union sicherzustellen. Laut Artikel 14 der Frontex-VO, der die Maßnahmen der Agentur an den Außengrenzen behandelt, muss die Agentur zugleich stets ,im Einklang mit dem einschlägigen Unionsrecht und Völkerrecht, einschließlich des Grundsatzes der Nichtzurückweisung“ handeln. Ergänzend bestimmt Artikel 21 der Frontex-VO, dass Frontex-Einsatzteams ihre Aufgaben und Befugnisse „unter uneingeschränkter Achtung der Grundrechte einschließlich des Rechts auf Zugang zum Asylverfahren, und der Menschenwürde“ ausüben müssen. $^{29}$

Weitere Orientierung für die Tätigkeit von Frontex und damit auch die Ausbildung bietet der Schengener Grenzkodex, der 2016 neu gefasst wurde und die Modalitäten des Grenzübergangs festschreibt. ${ }^{30}$ Der Grenzkodex

28 Anna Mrozek, Grenzschutz als supranationale Aufgabe, 2013, S. 307/308.

29 Vgl. zur Diskussion der materiellen Standards für Einsätze Simon Neumann, Die Europäische Grenzschutzagentur Frontex, 2014, S. 145 ff.

30 Verordnung (EU) 2016/399 des Europäischen Parlaments und des Rates v. 9. März 2016 über einen Gemeinschaftskodex für das Überschreiten der Grenzen durch Personen, Abl. EU L 77/1. 
legt z.B. in Erwägungsgrund 7 ausdrücklich fest, dass Grenzübertrittskontrollen so durchgeführt werden sollen, dass die menschliche Würde in vollem Umfang gewahrt wird. Damit ist es der Agentur Frontex etwa versagt, Einsätze zu planen, die zum Ziel haben, Menschen ohne Prüfung ihrer Schutzbedürftigkeit an der Grenze - zu Wasser oder zu Land - einfach abzuweisen. Die Grenzschutzbeamten sind nicht dazu ausgebildet, schon an der Grenze selbst eine Vorprüfung des Asyl- bzw. Schutzbegehrens vorzunehmen.

Gleichzeitig aber ordnet Erwägungsgrund 8 die „Migration“ kategorial schon als Bedrohung der Sicherheit der Außengrenzen ein. Hier wird deutlich, dass es aus der Sicht der Agentur einen unmittelbaren Zusammenhang zwischen Migration und einer Bedrohung der EU gibt. Migration wird so als Teil einer Gefährdungslage - ähnlich der schweren, organisierten Kriminalität - verstanden, auf die Frontex mit den Mitteln der Gefahrenabwehr zu reagieren hat. Eine Sicherheitsbehörde wie Frontex muss nun auf ein als Bedrohung festgelegtes Szenario „Migration“ seinem Auftrag entsprechend mit Gefahrenabwehr reagieren und so ein Bedrohungsszenario effektiv beenden. Migration und Grenzübertritt werden so zu einer Gefahr, die die Agentur zu verhindern hat, während sie zugleich auf Menschenrechte und die entsprechenden Garantien aus dem Flüchtlingsrecht verpflichtet ist. Ein kaum lösbarer Konflikt innerhalb des aktuellen politischen Auftrages, der sich ebenso ambivalent in den Rechtsgrundlagen für das Handeln der Agentur wiederfindet.

Das politische Auftragsszenario für Frontex ist durch die Agentur selbst nicht verhandelbar, denn sie ist formal kein politischer Akteur, der das Migrationsrecht der EU und damit die eigene Handlungs- und Rechtsgrundlage mit ausgestaltet. Hierfür sind die politischen Organe wie die Europäische Kommission, das Europäische Parlament und der Rat sowie die EUMitgliedstaaten im Rahmen des integrierten Ansatzes verantwortlich. Zugleich wird aber der rechtlich unabhängigen Agentur im Kontext der aufgezeigten Ambivalenz ein weiter Handlungsspielraum eröffnet, den sie über die eigens erstellten Risiko- und Gefährdungsanalysen, sowie die darauf basierenden Einsatzpläne selbst ausgestaltet und den mitgliedstaatlichen Behörden im Rahmen gemeinsamer Aktionen vorgibt. 


\section{5. ÜBERWACHUNG DER SEEAUSSENGRENZEN DURCH DIE EU}

Welche Rechtspflichten werden nun für das Grenzmanagement von Frontex durch die maßgeblichen Rechtsgrundlagen mit Blick auf die EUSeeaußengrenzen generiert? Die Situation an den See-Außengrenzen der EU spiegelt die geschilderte Ambivalenz im Auftrag von Frontex in besonderer Weise wieder. Anders als bei dem Überschreiten von Landgrenzen, wo die Schutzsuchenden auch bei Richtungsänderungen wegen Zurückweisungen o.Ä. zumindest grundsätzlich ,sicheren Boden“ unter den Füßen haben und sich potentiell umorientieren können, geht es angesichts der schwierigen Bedingungen auf Hoher See, unabhängig von den konkreten Perspektiven auf einen rechtlichen Schutzstatus, für die Schutzsuchenden aufgrund erhöhter Vulnerabilität oft um Leben und Tod. Zudem besteht auf See eine sehr eingeschränkte Öffentlichkeit, die folglich die dortigen Praktiken des Grenzschutzes nur schwierig beobachten, nachvollziehen und offenlegen kann, so dass sämtliche Akteure in einem relativ beobachtungsfreien Raum handeln.

Ziel der Unionspolitik ist auch auf See in logischer Konsequenz des EU-Gesamtansatzes in erster Linie die wirksame Grenzüberwachung, die entsprechend den Kontrollen an den Landgrenzen - vorrangig der „Verhinderung unbefugter Grenzübertritte“ dienen soll, ${ }^{31}$ also solchen Grenzübertritten, die nicht rechtlich durch Visum o. Ä. ausdrücklich genehmigt sind. Gemäß der hier maßgeblichen Seeaußengrenzen-Verordnung der EU von 2014 (VO 656/2014) ${ }^{32}$ dienen Einsätze auf See unter der Leitung von Frontex aber auch dem „Abfangen von Schiffen, die mutmaßlich ohne Grenzkontrolle in die EU einzulaufen versuchen“. Damit gerät nicht nur der Moment des Grenzübertritts selbst in den Fokus der Tätigkeit der Agentur, sondern schon vorbereitende Handlungen, über deren tatsächliche oder

31 Erwägungsgrund 1 der Seeaußengrenzen-Verordnung.

32 Verordnung (EU) Nr. 656/2014 zur Festlegung von Regelungen für die Überwachung der Seeaußengrenzen im Rahmen der von der Europäischen Agentur für die operative Zusammenarbeit an den Außengrenzen der Mitgliedstaaten der Europäischen Union (Frontex) koordinierten operativen Zusammenarbeit (Seeaußengrenzen-Verordnung). 
rechtliche Einordnung eben schon „Mutmaßungen“ der handelnden Grenzschützer ausdrücklich zum Einschreiten zwecks Mobilitätsverhinderung ausreichen.

Der Versuch das Territorium eines EU-Mitgliedstaats zu erreichen und dort um Schutz zu ersuchen, erfolgt gerade an den Seegrenzen - auch mangels Anspruch auf Visa aus humanitären Gründen ${ }^{33}$ - oft ohne Einreiseerlaubnis, also ohne Autorisierung des Zielstaates ${ }^{34}$ und wird so aus der Sicht von Frontex zur irregulären Handlung, die zudem noch an die „illegale“ Handlung einer Schleusung bzw. Fluchthilfe geknüpft ist, die generell unter dem Stichwort „organisierte Kriminalität“ behandelt wird. Der Versuch des Grenzübertritts auf dem Seeweg zur Erlangung von Schutz wird so zur Neben- oder Teilhandlung einer als kriminell angesehenen Tat umgedeutet und folglich selbst als Bedrohung von Sicherheit in der EU klassifiziert. Mithin werden schutzsuchende Migrant*innen als Teil einer Gefährdungslage für die EU wahrgenommen und in der Folge kategorisch als Sicherheitsproblem behandelt.

Gleichzeitig hat die Seeaußengrenzen-Verordnung auch eine andere Rechtsdimension, denn sie definiert als weiteres Ziel der Unionspolitik, dass „ein Beitrag zur Gewährleistung des Schutzes und der Rettung von Menschenleben“ geleistet werden soll und etabliert damit „Such- und Rettungseinsätze für Menschen in Seenot“, die „,in Situationen erfolgen, die sich unter Umständen während einer Grenzüberwachungsaktion auf See ergeben“ “ ${ }^{35}$ Verwiesen wird dabei auf das Völkerrecht, also die hier insbesondere einschlägigen seerechtlichen Normen des oben bereits analysierten SRÜ (vgl. I.), sowie ausdrücklich auf die weiteren Vorschriften der Seeaußengrenzen-Verordnung ${ }^{36}$.

Diese sieht vor, dass „Vorkehrungen“ für „möglicherweise erforderlich werdende Such- und Rettungsaktionen" getroffen werden. ${ }^{37}$ Schon mit

33 EuGH (Urt. v. 07. März 2017) Rechtssache C-638/16 PPU, Rn. 43.

34 Vgl. zur Politik der Mobilitätsverhinderung durch Visapolitik den Beitrag von Thea Kirsch in diesem Band.

35 Art. 4 b) Frontex-VO.

36 Siehe hierzu auch die weiteren Verpflichtungen aus Erwägungsgrund 8 und 9 der Seeaußengrenzen-Verordnung.

37 Erwägungsgrund 1 der Seeaußengrenzen-Verordnung. 
Blick auf die Formulierung „möglicherweise erforderlich“ erweist sie sich als nur sekundäre Aufgabenzuweisung an die Agentur, deren Fokus auf ordnungspolitische und nicht humanitäre Ziele gerichtet ist.

Und während Art. 4 Abs. 1 der Seeaußengrenzen-Verordnung ausdrücklich bestimmt, dass der Grundsatz der Nichtzurückweisung (in Übereinstimmung mit Art. 33 GFK) verbindlich gilt, wonach Schutzsuchende entsprechend der gefestigten Rechtsprechung des Europäischen Gerichtshofs für Menschenrechte (EGMR) - nicht in Staaten oder Bürgerkriegssituationen aus- oder zurückgewiesen werden dürfen, in denen sie der ernsthaften Gefahr ausgesetzt wären, gefoltert, unmenschlich behandelt oder getötet zu werden, ${ }^{38}$ beinhalten die weiteren Absätze des Art. 4 der Seeaußengrenzen-Verordnung Ausnahmen von dieser generellen Pflicht. Die beteiligten Einsatzkräfte sollen auf der Grundlage des Frontex-Einsatzplans und der darin enthaltenen Bewertung, die gestützt ist auf Informationen aus dem betreffenden Drittstaat selbst, EU-Behörden und Internationalen Organisationen, im eigenen Ermessen entscheiden.

Relevant ist am Ende die Beurteilung der ,allgemeinen Lage“ im Drittstaat (Art. 4 Abs. 2). So soll beispielsweise die Möglichkeit der Ausschiffung (,an Land bringen“) von abgefangenen Schutzsuchenden in einem Drittstaat dann ohne Verstoß gegen den Grundsatz der Nichtzurückweisung möglich sein, wenn zwischen der EU und dem Drittstaat „Vereinbarungen und Projekte im Bereich Migration und Asyl bestehen, die im Einklang mit dem Unionsrecht und mit Mitteln der Union durchgeführt werden“. Dies enthält sozusagen die Vermutung für eine positive Bewertung der allgemeinen Lage in einem Drittstaat, wenn Kooperationen mit diesem Drittstaat bestehen. Diese Vorschrift könnte also derart gedeutet werden, dass jede der vielen, mit osteuropäischen und nordafrikanischen Staaten bestehenden

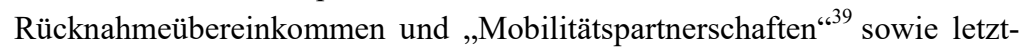

38 EGMR (Große Kammer), Ahmed gegen Österreich (Urteil vom 17. Dezember 1996), Beschw. Nr. 25964/94.

39 Steffen Angenendt, Migration, Mobilität und Entwicklung, SWP-Studie, 2012, S. 5 ff. und Kleine Anfrage Fraktion DIE LINKE zu Mobilitätspartnerschaften und das Grenzmanagement der Europäischen Union, BT Drs. 18/6868, 2015 und weiter Christian Jakob/Simone Schlindwein, Diktatoren als Türsteher Europas, 2017, S. 21 ff. 
lich jede zivile, polizeiliche oder militärische Kooperation, die einen Bezug zu Asyl und Migration aufweist, als Rechtfertigung für eine Ausnahme vom Grundsatz der Nichtzurückweisung zu verstehen ist. Die Abkommen zwischen der EU und Drittstaaten sind formal im Einklang mit dem Unionsrecht, denn die EU als Gesamtverband kann im Rahmen vorgesehener Verfahren jedwede Kooperation eingehen und auch Frontex selbst hat als Agentur die Möglichkeit Verwaltungsabkommen bzw. sogenannte „Working Arrangements" mit Behörden von Drittstaaten einzugehen, die ebenfalls für Grenzschutz zuständig sind (Art. 54 Frontex-VO). ${ }^{40}$

So ist es zwar einerseits eine zu beobachtende Praxis im Mittelmeer, dass Schutzsuchende auf Hoher See zum Teil aus Seenot gerettet und von Schiffen des Grenzschutzes oder gar des Militärs aufgenommen, ${ }^{41}$ zum Teil aber auch in nicht unerheblichem Umfang ohne die Chance der Einleitung eines rechtsstaatlichen Verfahrens oder auch nur der kurzfristigen Ermöglichung eines gesicherten Zustandes, schon auf See zurückgewiesen werden, sei es durch Abdrängen und Aufhalten von Schiffen ${ }^{42}$ oder das unmittelbare Verbringen von aufgegriffenen Menschen zurück in unsichere Krisenregionen wie Libyen, wo eine zerbrochene Staatlichkeit einer kontrollierbarmenschenwürdigen Behandlung entgegensteht, vielmehr Folter, Vergewaltigung, menschenunwürdige Unterbringung - mithin schwerste Menschenrechtsverletzungen - einer Vielzahl von Schutzsuchenden in Libyen droht bzw. wiederfährt. ${ }^{43}$

40 Zum Verständnis der „Grenzschützer als Diplomaten“ vgl. Christian Jakob/Simone Schlindwein, Diktatoren als Türsteher Europas, 2017, S. 172 ff.

41 Frontex Risk Analysis 2018, S. 6, 9.

42 Wissenschaftlicher Dienst des BT, Seenotrettung im Mittelmeer, WD 2 - 3000 013/18, S. 4, unter Verweis auf: Border Criminologies, „Italy Strikes Back Again: A Push-back's Firsthand Account“ (15. Dezember 2017), verfügbar unter https://www.law.ox.ac.uk/research-subject-groups/centre-criminology/centrebor der-criminologies/blog/2017/12/italy-strikes, „The Case for Italy's Complicity in Libya Push-Backs“ (4. November 2017), verfügbar unter: https://www.news deeply.com/refugees/community/2017/11/24/the-case-for-italys-complicity-in -libya-push-backs (beide zuletzt aufgerufen am 18.01.2019).

43 Amnesty International, Libya is full of cruelty. Stories of abduction, sexual violence and abuse from migrants and refugees, 2015, S. 11 ff.; UNHCR und 
In einem Rechtsgutachten vom Februar 2018 hat der Wissenschaftliche Dienst des Deutschen Bundestages in diesem Kontext deutlich ausgeführt, dass es für einen Verstoß gegen das Refoulement-Verbot nicht darauf ankomme, ob dies durch Tun oder durch Unterlassen (wie z.B. Abwarten) geschehe, da die GFK in Art. 33 ausdrücklich vorgibt, dass ein Staat bzw. Hoheitsträger „,in keiner Weise“ eine Aus- oder Zurückweisung vornehmen oder geschehen lassen darf. ${ }^{44}$ Dies ist schon deswegen gerade aus deutscher Sicht bedeutsam, da deutsche Behörden das größte Kontingent der Grenzschutzagentur Frontex stellen und somit auf die Gesamtstruktur der Operationen gewichtigen Einfluss haben. ${ }^{45}$

\section{6. ÜBERWACHUNGSDIMENSIONEN UND KAPAZITÄTEN}

Die Überwachung der EU-Außengrenzen erfolgt vielgestaltig und mittels unterschiedlicher Akteure und Techniken. Zunächst gibt es die unmittelbare

UN Support Mission in Libya, Detained and Dehumanised. Report on Human Rights abuses against migrants in Libya, 2016; UNHCR, Mixed Migration Trends in Libya, 2017, S. 148; ProAsyl, Menschenrechte über Bord! Warum Europas Kooperation mit Libyen so schändlich ist, 22.08.2017, https: //www.proasyl.de/hintergrund/menschenrechte-ueber-bord-warum-europas-koo peration-mit-libyen-so-schaendlich-ist/ und Dominik Peters/Maximilian Popp, Für Flüchtlinge die Hölle - für die EU ein Partner, http://www.spiegel. de/politik/ausland/libyen-die-hoelle-fuer-fluechtlinge-ein-partner-fuer-die-eu-a1202364.html (12.04.2018); Zu der Situation vor dem sog. „Arabischen Frühling“ 2011 vgl. Britta Leisering, Menschenrechte an den Europäischen Außengrenzen, 2016, S. $179 \mathrm{f}$.

44 Wissenschaftlicher Dienst des BT, Seenotrettung im Mittelmeer, WD 2 - 3000 013/18, S. 10.

45 Vgl. Spiegel online v. 05.04.2018, unter Berufung auf einen internen Bericht der Bundespolizei, http://www.spiegel.de/politik/deutschland/deutschland-stelltgroesstes-kontingent-fuer-frontex-a-1201351.html; Demnach sind 114 deutsche Beamte für Frontex im Einsatz, was einen Anteil von 21.8 Prozent vom Gesamtpersonal darstellt. 
Überwachung durch Einsätze vor Ort, die von der Agentur Frontex organisiert und koordiniert werden. Federführend ist vor Ort zwar grundsätzlich der Mitgliedstaat, an dessen Grenzen ein Einsatz stattfindet (Einsatzmitgliedstaat), doch die Grundlage für jegliches grenzrelevantes Handeln in einem von Frontex geleiteten Einsatz ist der verbindliche FrontexEinsatzplan. Die Kapazitäten von Frontex zur Stärkung der maritimen Präsenz wurden über die Jahre erheblich erweitert und Einsätze auf See verstetigt, wie die Operationen Triton und Poseidon. Operation Poseidon fokussiert die Grenzüberwachung des Seegebiets rund um Griechenland bis zur Türkei und soll neben der Organisation von Grenzschutz und Rückführung auch der Seenotrettung dienen. Die von 2014 bis Anfang 2018 durchgeführte Operation Triton umfasste die Küstengewässer rund um Italien und Malta und das Seegebiet südlich von Sizilien. Der Übergang von Mare Nostrum auf Triton bedeutete schon angesichts des kleineren Einsatzgebietes eine Reduzierung der Seenotrettungskapazitäten, doch immerhin sollten die geretteten grundsätzlich in italienische Häfen gebracht werden. Das ändert sich nun ausdrücklich durch die neue Frontex-Operation Themis, die zum 1. Februar 2018 Triton ersetzt. ${ }^{46}$ Während einerseits die Patroullientätigkeit ausgeweitet wird, soll ab jetzt von Fall zu Fall entschieden werden, in welchen Hafen Schutzsuchende verbracht werden. Ob dies nur europäische oder auch außereuropäische Häfen sein können, bleibt vorerst offen, wenngleich nach Aussagen der Agentur Frontex nur weitere Zielhäfen in Kroatien, Malta und Spanien im Blick seien. ${ }^{47}$ Der Einsatzradius verringert sich jedenfalls spürbar und soll lediglich die 30 Meilen-Zone rund um das italienische Festland umfassen. Diese abermalige Reduzierung hat automatisch eine Verringerung der Seenotrettungstätigkeit zur Folge.

Mit der neuen Operation Themis ändert sich zugleich der Auftrag, denn nun soll Frontex, ganz im Sinne der bereits geschilderten Betonung der sicherheitsrechtlichen Dimension der Tätigkeit der Agentur, die Gewässer

46 Frontex Press Release https://frontex.europa.eu/media-centre/news-release/ frontex-launching-new-operation-in-central-med-yKqSc7 (letzter Aufruf am 18.01.2019).

47 So nachlesbar unter Verweis auf den Frontex Sprecher Ton van Lierop: https://openmediahub.com/2018/03/12/eu-sea-mission-themis-protect-europemigration/ (letzter Aufruf am 18.01.2019). 
primär mit Blick auf grenzüberschreitende Kriminalität, also Waffen- und Drogenschmuggel oder auch Terrorismus überwachen. Auch Migration wird so weiter versicherheitlicht.

Diese Neuausrichtung des Auftrages kann schon verwundern, da es mit der EU Militäroperation EUNAVOR MED/Sophia, der den Menschenhandel und die Schleusernetzwerke im Mittelmeerraum zwischen Italien, Libyen und Tunesien „,bekämpfen“ soll, und dem NATO Einsatz Sea Guardian, der ebenfalls auf die „Bekämpfung“ von Waffenhandel und Schleppernetze ausgerichtet ist, aktuell bereits zwei umfangreiche militärische Sicherheitsoperationen im Mittelmeer gibt. ${ }^{48}$

Die verringerte operationelle Dimension von Frontex bedeute zwar weniger Präsenz zur Seenotrettung, aber nicht weniger Überwachung des Mittelmeerraums, denn einmal entsendet die Agentur in die betreffenden Mitgliedstaaten und Drittstaaten rund um das Mittelmeer Verbindungsbeamte, um für die bessere Überwachung der Außengrenzen zusätzliche Informationen zu sammeln und so Aufklärung über die Situation vor Ort in den (Mitglied-)Staaten zu erhalten, wie es Artikel 8 c) und Artikel 12 Frontex-VO als Aufgabe vorgibt.

Zudem arbeitet Frontex schon seit Jahren an einer Strategie zur effektiven „Externalisierung“ des EU-Grenzschutzes. Die Agentur hat aus Art. 54 Frontex-VO das Mandat im Rahmen von Arbeitsabkommen mit Grenzbehörden von Drittstaaten zu kooperieren, also z.B. Grenzbeamte anderer Staaten auszubilden, an Fahrzeugen und Geräten zu schulen und Grenzüberwachungssysteme aufzubauen. Derartige Kooperationen gibt es bereits mit 18 Staaten, darunter die Ukraine, Serbien, Mazedonien, Nigeria und die Türkei. Mit weiteren acht Staaten bestehen Verhandlungen, die unterschiedliche Stadien der Verbindlichkeit erreicht haben, darunter Marokko, Senegal, Ägypten, Brasilien, Tunesien und Libyen. ${ }^{49}$ Auch wenn die Ausrüstung und Ausbildung auswärtiger Dienste - wie z.B. im Fall der Küstenwache von Libyen - zumeist nur dazu dient, Fluchtbewegungen zu unterbinden und letztlich die EU-Schutzstandards durch Externalisierung des

48 Vgl. zur Debatte: Bundestag 19. Wahlperiode, 23. Sitzung, 22. März 2018, S. $2084 \mathrm{ff}$.

49 Zur Übersicht vgl. https://frontex.europa.eu/partners/non-eu-countries/ (letzter Aufruf am 18.01.2019). 
Grenzschutzes auf ausländische Behörden unterlaufen werden, bleibt Frontex durch diesen Kooperationsverbund in den südlichen Küstenregionen des Mittelmeeres folglich präsent und hat damit auch Einsicht in die Überwachung der dortigen See.

Überdies bestehen enge geheimdienstliche Kontakte von Frontex mit Diensten von Drittstaaten, wie insbesondere über die für den Mittelmeerraum besonders relevante, 2010 gegründete Africa-Frontex Intelligence Community (AFIC), die sich an bestehenden Modellen der Frontex Risk Analysis Networks (FRAN) mit osteuropäischen Staaten orientiert. ${ }^{50}$

Und last but not least gibt es die informationelle Überwachung des gesamten Mittelmeerraums durch die Installierung und stetige technische Verbesserung des Europäischen Grenzüberwachungssystems EUROSUR (European Border Surveillance System),${ }^{51}$ das auf Identifizierung und Verfolgung von Schiffen ausgerichtet ist, eine wesentliche Komponente im EU-Außengrenzschutz. Frontex steuert die Verknüpfung zwischen den Mitgliedsstaaten, die rund um das Mittelmeer ihre eigenen Überwachungszentren haben, dem Satellitenzentrum der EU (EUSC), der Europäischen Agentur für die Sicherheit des Seeverkehrs (EMSA) sowie der ebenfalls beteiligten Agentur Europol. In die Zusammenarbeit ist auch die militärische Operation EUNAVORMED/Sophia einbezogen, so z.B. am „Eurosur Fusion“ Projekt von Frontex. ${ }^{52}$ Durch Satellitenüberwachung und drohnengestützte Luftraumaufklärung sowie ein zentral vernetztes Schiffsmeldesystem, wird ein umfassendes Lagebild von Bewegungen auf dem Mittelmeer

50 Vgl. dazu Africa-Frontex Intelligence Community Joint Report 2016, S. 8 ff. und weiter Christian Jakob/Simone Schlindwein, Diktatoren als Türsteher Europas, 2017, S. 173.

51 Verordnung (EU) Nr. 1052/2013 des Europäischen Parlaments und des Rates vom 22. Oktober 2013 zur Errichtung eines Europäischen Grenzüberwachungssystems (EUROSUR).

52 Niels Frenzen, Satellite Imagery used by Frontex to detect and rescue migrant boats, https://migrantsatsea.org/tag/eurosur-fusion-services/ und Frontex Report on the functioning of Eurosur - part 1, Council Doc 6215/18 v. 15. Februar 2018, S. 10. 
möglich. ${ }^{53}$ Durch die Koordinierung der unterschiedlichen Kommunikations- und Datenaustauschsystemen sowie die Einbeziehung automatisierter Überwachungssysteme an den Außengrenzen und den grenznahen Bereich, wird ein hohes Level von Interoperabilität der Systeme erzeugt, das, auch mit Hilfe einer komplexen Berechnungssoftware, etwa Schiffsbewegungen größerer aber zunehmend auch kleinerer Schiffe und Boote auf dem Mittelmeer ,live“ sichtbar machen kann. ${ }^{54}$

Die südlichen Mitgliedstaaten der Europäischen Union, also Spanien, Portugal, Frankreich, Italien, Malta, Griechenland und Zypern, betreiben die satellitengestützte Plattform „Seepferdchen Mittelmeer“ („Seahorse Mediterranean"), das eine von der Europäischen Kommission mit initiierte und langjährig verhandelte Zusammenarbeit im grenzpolizeilichen Bereich mit Tunesien, Algerien und Ägypten intensiviert und so die nordafrikanischen Länder auch in das EU-Überwachungssystem Eurosur - und damit in den von Frontex gesteuerten Bereich des EU-Außengrenzschutzes - integriert. ${ }^{55}$ Laut der Europäischen Kommission beteiligen sich Tunesien, Alge-

53 Hierzu und zum Folgenden Joachim Stern/Timo Tohidipur, in v.Arnauld (Hrsg.), Europäische Außenbeziehungen, Enzyklopädie Europarecht Band 10, 2014, §14 Migration von Drittstaatsangehörigen, Rn. 51. Siehe auch den Abschlussbericht von Projekt PERSEUS - Protection of European seas and borders through the intelligent use of surveillance,wo es primär um die Erprobung der Interoperabilität der unterschiedlichen im Einsatz befindlichen Systeme geht, https://cordis.europa.eu/docs/results/261/261748/final1-

perseus_final_publishable_report.pdf (letzter Aufruf am 18.01.2019), dort insbesondere S. 36 f.

54 Niels Frenzen, Satellite Imagery used by Frontex to detect and rescue migrant boats, https://migrantsatsea.org/tag/eurosur-fusion-services/ und Catherine Strupp, EU-Seeverkehrsbehörde will Flüchtlingsboote mit Drohnen aufspüren, EURACTIV v. 07.03.2016, https://www.euractiv.de/section/eu-innenpolitik/ news/eu-maritime-agency-gets-ready-to-use-drones-to-monitor-refugee-boats/ (letzter Aufruf am 18.01.2019).

55 Deutscher Bundestag, Kleine Anfrage von Andrej Hunko u.a. und der Fraktion DIE LINKE, Drucksache 18/11974, v. 1004.2017 und die Antwort der Bundesregierung Drucksache 18/12459 v. 18.05.2017. Vgl. auch Matthias Monroy, Durch die Hintertür: Anschluss Libyens an Europäische Überwachungssysteme, 
rien und Ägypten bereits und auch Libyen soll nun enger in die grenzpolizeiliche und militärische Überwachung des Mittelmeeres einbezogen werden. So haben italienische und libysche Sicherheitsbehörden im Januar 2018 ein gemeinsames Kontrollzentrum in Rom in Betrieb genommen und Libyen ist zudem am System SMART - „Service-oriented infrastructure for Maritime Traffic tracking“ - des italienischen Militärs beteiligt, ebenso wie die NATO und Frontex. Libysche Grenzbehörden waren dafür zuvor im Rahmen der EU-Militärmission EUNAVORMED geschult worden.

Durch diese enge Verzahnung der polizeilichen, geheimdienstlichen und militärischen Apparate werden nicht nur Tendenzen effektiver Militarisierung der EU-Grenzen verstärkt, sondern zugleich auch ein sehr hohes operatives und technisches Niveau der Überwachung des Mittelmeerraums erreicht.

\section{VERPFLICHTUNG VON FRONTEX ZUR AKTIVEN SEENOTRETTUNG}

Das mittlerweile erreichte Niveau der technischen und informationellen Überwachung des Seeverkehrs im Mittelmeer dient im ersten Zugriff einem originär grenzpolizeilichen Zweck, der möglichst umfassenden „Aufdeckung“ und „Abwehr“ unerwünschter Migration über das Mittelmeer, generiert aber zugleich im Zusammenspiel mit der dargestellten Rechtslage des Seerechts ein Pflichtenszenario für die Grenzschutzagentur Frontex, das dem angestrebten Zweck der Abwehr diametral entgegensteht.

Frontex ist als Agentur zunächst ,nur“ eine Behörde, die den politischen Auftrag der EU vollzieht, der durch die rechtsetzenden Organe in Rechtsnormen und damit Arbeitsvorgaben für die Agentur überführt wurde. Doch basiert das typische Handlungsspektrum von Verwaltung auf der eigenverantwortlichen Ausgestaltung von Ermessenspielräumen und folgt damit durchaus einer Eigendynamik, die durch die äußeren Rahmenbedingungen bestimmt wird, gerade durch ihre spezifische Verrechtlichung. Die

Cilip-blog, 19. Januar 2018, https://www.cilip.de/2018/01/19/durch-diehintertuer-anschluss-libyens-an-europaeische-ueberwachungssysteme/\#more13651 (letzter Aufruf am 18.01.2019). 
über die Jahre voranschreitende, politisch ausdrücklich forcierte Ausweitung des Auftrages der Agentur, deren Tätigkeit sich immer mehr auch auf organisierte Kriminalität konzentriert, einhergehend mit der stetigen Aufstockung des Budgets und der Personal- und Sachausstattung, verdeutlicht die Wichtigkeit des Außengrenzschutzes für die EU und damit die Bedeutung der Agentur Frontex.

Mit der Betonung einer gemeinsamen Verantwortung von Mitgliedstaaten und Agentur, reklamiert die Agentur die Rolle des Managers des integrierten Grenzschutzes an den EU-Außengrenzen für sich. Den dargelegten Kompetenzen der Agentur bei der Organisation und Koordination der Operationen im Mittelmeer folgen rechtliche Verantwortlichkeiten und Handlungspflichten.

Der sicherheitsrechtliche Auftrag der Agentur dominiert dabei alle ihre Operationen. Ein operativer Wechsel der Agentur, zu einer proaktiven Küstenwache, die vorrangig Seenotrettung koordiniert und betreibt, würde zwar eine komplette administrative Neuausrichtung bedeuten, ist aber rechtsstaatlich durchaus geboten. Durch die Präsenz vor Ort im Rahmen der Operation Themis und der anderen Operationen zuvor, besteht i.S.d. Art. 98 Nr. 1 a) SRÜ die unbedingte Pflicht zur Hilfe bei Lebensgefahr für Menschen auf See. Diese Pflichten wurden im Rahmen der Frontex-Einsätze auch grundsätzlich anerkannt und so über die letzten Jahre Menschen gerettet. Doch interessant wird in besonderer Weise die Verpflichtung darüber hinaus, wenn i.S.d. Art. 981 b) „Kenntnis von der Hilfsbedürftigkeit“ erlangt wird - gerade hier spielt die dargestellte Überwachungsdichte und die informationelle Koordinierung und Zentralisierung sowie die Interoperabilität der durch die unterschiedlichen Akteure genutzten Datensysteme eine entscheidende Rolle. Die politisch und technisch angestrebte, möglichst umfassende Kenntnis von der Situation in den Staaten, aus denen die Schutzsuchenden ins Mittelmeer aufbrechen, über die satelliten- und drohnengestützte Verfolgung von Booten aller Art auf See bis hin zur systematischen Einbindung der Erkenntnisse militärischer Operationen von EU und NATO, führt eben auch zur immer umfassenderen Kenntnis über Hilfsbedürftigkeit auf See, die entsprechend Art. 981 b) verpflichtet ,so schnell wie möglich Personen in Seenot zu Hilfe zu eilen“. Frontex generiert sowohl Kenntnisse über die Küstenabschnitte der außereuropäischen Drittstaaten wie auch über die Küstenregionen Europas. Hinter dieser ausdrücklichen see- und menschenrechtlichen Pflicht, auf die auch in den Erwä- 
gungsgründen 8 und 9 der für Frontex grundlegenden SeeaußenrechtsVerordnung verwiesen wird, treten anderweitige politische Aufträge der Akteure hinten an, ebenso wie die Frage, ob und inwieweit Betroffene Aussichten auf einen Schutzstatus in der EU haben oder nicht. Dies bleibt rechtlichen Verfahren überlassen, die nicht auf See stattfinden können, weshalb den Betroffenen nach dem Hirsi-Urteil des EGMR Zugang zum europäischen Territorium zu gewähren ist. Auf der See geht es zunächst um jedes Einzelschicksal, jede einzelne Person, die unabhängig von den möglichen Statusperspektiven, um ihr Überleben auf See kämpft und unabhängig von ihrer konkreten Motivation zur Überfahrt zu retten ist. In der Konsequenz wird eine positive Pflicht zur Seenotrettung, ja sogar ein proaktives Operationserfordernis, für alle an Frontex Operationen beteiligten Schiffe der Mitgliedstaaten generiert und eine Pflicht, private Seenotrettung nicht durch Sanktionen oder deren Androhung zu verhindern.

Eine Verknüpfung von Intensivierung der Überwachung und steigender Verpflichtung zur Seenotrettung ist eine für die Sicherheitsbehörden sicher nicht intendierte Folge der Migrations- und Flüchtlingspolitik. Dieser Herausforderung muss sich nun gerade eine organisatorisch immer wirkmächtiger agierende Agentur Frontex stellen und sich so an ihren eigenen, zuvor dargelegten Rechtspflichten aus der Frontex-VO, der Seeaußenrechtsverordnung mit ihren Verweisen auf das zwingend einzuhaltende Seerecht messen lassen.

Geschieht dies nicht, liegt hierin nicht nur ein einfacher, wenngleich deutlicher Verstoß gegen Europa- und Völkerrecht, sondern vielmehr auch eine systematische Verabschiedung von rechtsstaatlichen Ansprüchen der $\mathrm{EU}$, mithin eine grundlegende Verabschiedung vom eigenen Wertekanon des Art. 2 EUV. So würde sich letztlich das Gesamtkonzept der sich als friedensstiftend und kosmopolitisch verstehenden EU einer viel fundamentaleren Kritik aussetzen.

\section{SCHLUSSBETRACHTUNGEN}

Der Beitrag hat verdeutlicht, dass die Agentur Frontex in ihrer bisherigen Tätigkeit einen eindeutigen Schwerpunkt auf die Grenzkontrolle und die Abwehr von Migration legt. In den Rechtsgrundlagen von Frontex ist dies durchaus angelegt, indem Migration dort als Sicherheitsproblem verhandelt 
wird. Durch diese Linse betrachtet Frontex die Migrationsbewegungen einseitig als Bedrohung für die Europäische Union. Mit dieser Zweckrichtung in Konflikt geraten aber gleichsam die menschenrechtlichen Verpflichtungen, an die die Agentur gebunden ist und die sich in ihrem Falle sogar zu einer proaktiven Verpflichtung zur Seenotrettung verdichten, weil Frontex durch seine Grenzkontrolltätigkeiten ein umfassendes Wissen über die Migrationsrouten erhält. Diese Verpflichtung würde konsequenterweise darauf hinauslaufen, dass die grenzkontrollierende Funktion von Frontex in den Hintergrund gedrängt wird. Ob es überhaupt sinnvoll ist einer Agentur, die bisher vor allem migrationsabwehrende Strategien verfolgt hat, zu einer proaktiven Seenotrettung zu verpflichten ist eine politische Frage.

Denn trotz der rechtspolitischen Perspektive auf eine proaktive Seenotrettung ist es ebenso klar - und das verdeutlichen auch die politischen Konflikte um die Seenotrettung -, dass die derzeitige Politik der Europäischen Union keineswegs einen solchen humanitären Ansatz präferiert. Charles Heller und Lorenzo Pezzani haben darauf hingewiesen, dass Migrant*innen die auf der See sterben keines „natürlichen Todes“ oder infolge einer „unvorgesehenen Katastrophe“" sterben, sondern dass die Bootsunglücke die Folge einer ,strategischen Nutzung der See ${ }^{\text {“56 }}$ durch die Europäische Union sind. Auf der See entfalte sich eine im Sinne Foucaults biopolitische Regierungsweise, die das Leben der einen aktiv schütze - das der eigenen Staatsangehörigen - und das Leben der anderen durch eine bewusste Unterlassung der Rettung zerstöre. ${ }^{57}$ Der Rechtswissenschaftler Itamar Mann spricht in diesem Zusammenhang von „maritimie legal black holes“, die extra-legale Praktiken normalisieren und das Recht auf Leben der Migrant*innen nicht berücksichtigt. ${ }^{58}$ Über die Wahrung der internationalen Rechtspflichten hinaus machen diese Einsichten daher deutlich, dass eine menschenwürdige

56 Charles Heller/Lorenzo Pezzani, Liquid Traces. Investigating the Deaths of Migrants at the EU's Maritime Frontier, in: de Genova, Nicholas (Hrsg.) The Borders of „Europe“. Autonomy of Migration, Tactics of Bordering, Durham/London 2017, S. 95 - 119, hier S. 96.

57 Ebd., S. 108.

58 Itamar Mann, Maritime Legal Black Holes: Migration and Rightlessness in International Law, European Journal of International Law, Vol. 29, Issue 2, 2017, S. $347-372$. 
Seenotrettungspolitik nicht alleine in der Arena des Rechts erkämpft werden kann, sondern es eines politischen Wandels bedarf, der die rechtlichen Vorgaben auch in eine konkrete politische Kultur und Praxis übersetzt.

\section{LITERATUR}

Africa-Frontex Intelligence Community Joint Report 2016

Amnesty International, Libya is full of cruelty. Stories of abduction, sexual violence and abuse from migrants and refugees, 2015

Angenendt, Steffen, Migration, Mobilität und Entwicklung, SWP-Studie, 2012

Cuttitta, Paolo, Zwischen De- und Repolitisierung. Nichtstaatliche Search and Rescue-Akteure an der EU-Mittelmeergrenze. In: Hess, Sabine u.a. (Hrsg.) Der lange Sommer der Migration. Grenzregime III, Berlin/Hamburg 2017, S. 115-125

Ewert, Linda, „Suchen. Retten. Legalisieren.“ Chancen und Grenzen zivilgesell-schaftlicher Seenotrettung am Beispiel der Organisation Sea Watch. In: Hess, Sabine u.a. (Hrsg.) Der lange Sommer der Migration. Grenzregime III, Ber-lin/Hamburg 2017, S. 126-136

Frenzen, Nils, Satellite Imagery used by Frontex to detect and rescue migrant boats, https://migrantsatsea.org/tag/eurosur-fusion-services/

Frontex Report on the functioning of Eurosur - part 1, Council Doc $6215 / 18$ v. 15 . Februar 2018

Frontex Risk Analysis 2018

Hathaway, James C. / Foster, Michelle, The Law of Refugee Status, 2nd ed 2014

Hathaway, James C./ Storey, Hugo, What is the Meaning of State Protection in Refugee Law? A Debate. International Journal of Refugee Law, Volume 28, Issue 3, 1 October 2016, S. 480-492

Heller, Charles / Pezzani, Lorenzo, Liquid Traces. Investigating the Deaths of Migrants at the EU's Maritime Frontier, in: de Genova, Nicholas (Hrsg.) The Borders of "Europe". Autonomy of Migration, Tactics of Bordering, Durham/London 2017, S. 95 - 119

Internation Organisation of Migration (IOM), Fatal Journeys. Volume 3 Part 1. Improving Data on Missing Migrants, Genf, 2017 
Jakob, Christian / Schlindwein, Simone, Diktatoren als Türsteher Europas, 2017

Juncker, Jean-Claude, Präsident der EU-Kommission, Rede zur Lage der Union 2017, Brüssel, 13. September 2017, vgl. http://europa.eu/ rapid/press-release_SPEECH-17-3165_de.htm (letzter Aufruf am 18.01. 2019)

Macron, Emmanuel, Rede von Staatspräsident Macron an der Sorbonne Initiative für Europa, Paris 26. September 2017, S. 5, https://de. ambafrance.org/Initiative-fur-Europa-Die-Rede-von-Staatsprasident-Ma cron-im-Wortlaut (letzter Aufruf am 18.01.2019)

Mann, Itamar, Maritime Legal Black Holes: Migration and Rightlessness in International Law, European Journal of International Law, Vol. 29, Issue 2, S. $347-372,2017$

Monroy, Matthias, Durch die Hintertür: Anschluss Libyens an Europäische Überwachungssysteme, Cilip-blog, 19. Januar 2018, https://www.cilip. de/2018/01/19/durch-die-hintertuer-anschluss-libyens-an-europaeischeueberwachungssysteme/\#more-13651 (letzter Aufruf am 18.01.2019)

Mrozek, Anna, Grenzschutz als supranationale Aufgabe, 2013

Neumann, Simon, Die Europäische Grenzschutzagentur Frontex, 2014

Oppermann, Thomas/Classen, Claus Dieter / Nettesheim, Martin, Europarecht, 8. Aufl., München 2018

Peters, Dominik / Popp, Maximilian, Für Flüchtlinge die Hölle - für die EU ein Partner, http://www.spiegel.de/politik/ausland/libyen-die-hoellefuer-fluechtlinge-ein-partner-fuer-die-eu-a-1202364.html (12.04.2018)

ProAsyl, Menschenrechte über Bord! Warum Europas Kooperation mit Libyen so schändlich ist, 22.08.2017, https://www.proasyl. de/hintergrund/menschenrechte-ueber-bord-warum-europas-kooperation -mit-libyen-so-schaendlich-ist/

Rah, Sicco, Asylsuchende und Migranten auf See. Staatliche Rechte und Pflichten aus völkerrechtlicher Sicht, Hamburg Studies in Maritime Affairs 15, Ber-lin/Heidelberg 2009

Schmid, Thomas, „Aus den Augen, aus dem Sinn“ - Flüchtlinge und Migranten an den Rändern Europas. Die zentrale Mittelmeerroute, HeinrichBöll-Stiftung, Berlin 2016

Stern, Joachim / Tohidipur, Timo, in v.Arnauld (Hrsg.), Europäische Außenbeziehungen, Enzyklopädie Europarecht Band 10, 2014, §14 Migration von Drittstaatsangehörigen 
Strupp, Catherine, EU-Seeverkehrsbehörde will Flüchtlingsboote mit Drohnen aufspüren, EURACTIV v. 07.03.2016, https://www.euractiv.de/sec tion/eu-innenpolitik/news/eu-maritime-agency-gets-ready-to-use-drones -to-monitor-refugee-boats/ (letzter Aufruf am 18.01.2019)

UNHCR und UN Support Mission in Libya, Detained and Dehumanised. Report on Human Rights abuses against migrants in Libya, 2016

UNHCR, Mixed Migration Trends in Libya, 2017

Wissenschaftlicher Dienst des Bundestages, Die völkerrechtliche Pflicht zur Seenotrettung, WD 2 - 3000-053/17, 2017

Wissenschaftlicher Dienst des Bundestages, Der italienische Verhaltenskodex für private Seenotretter im Mittelmeer Völker-, europa- und strafrechtliche AspekteWD 2 - 3000 - 068/17, 2017

Wissenschaftlicher Dienst des Bundestages, Seenotrettung im Mittelmeer, WD $2-3000-013 / 18,2018$

Wissenschaftlicher Dienst des Bundestages, WD 5 - 3000 - 124/18, 2018

Zagst, Lena, Bedenklicher italienischer Verhaltenskodex für Seenotrettung durch NGOs im Mittelmeer, Juwiss-Blog vom 15. Dezember 2017, https://www.juwiss.de/139-2017/ (letzter Aufruf am 18.01.2019) 
Leben nach der Grenze 



\section{How social media facilitate migration in Europe}

Sara Abbasi

'Opportunity': picture taken by a Syrian refugee in Belgium.

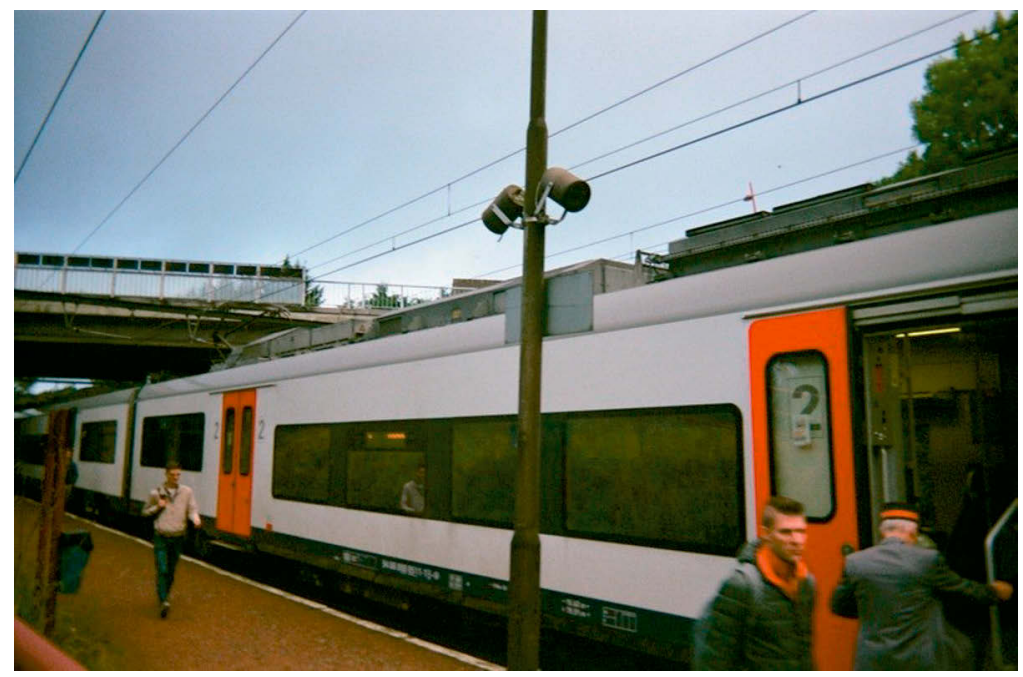

Source: refugeepostcards.com

Whilst visiting Istanbul with my mother in 2015, I experienced fright and apprehension after my mother was told that she was late for check-in and therefore couldn't board the plane back to London. The uncertainty of her return home and concern for her safety was all I could think about as I flew 
back to Copenhagen. What would she do by herself? Would she be safe in a foreign country? Would she be able to get home?

But these are questions refugees are asking themselves every day as they leave everything behind - everything they have ever known - in order to find solace and safety in a foreign, alien land. It is safe to say that the fear and anxiety I felt is pitiful when compared to the sentiments of people fleeing their states, often not by choice but due to the hopelessness of building a future at home or, worse, due to the fear of being killed.

Previously, the situation for refugees and migrants who moved abroad was made increasingly difficult, as they experienced detachment and isolation from their own communities of origin, with their existing social ties being cut. They had limited contact with their families and loved ones, as they kept in touch by sending letters, telegrams, and packages by mail, some of which could sometimes take months to arrive. Later, the boom in cheap international telephone calls served as social glue, enabling migrants and refugees to stay connected to their relatives and friends back home.

But since the rise of the internet, things have taken a whole new turn especially when it comes to communication. In today's network society, the speed and intensity of information flow has increased tremendously and is shaping the everyday lives of people. "[Social media] is very useful for refugees because it's one of the only ways to know what's going on in our country - by talking to friends and family, and by reading articles posted on the [Facebook] timeline. Most importantly, we can keep in contact with family to make sure they are safe. I mean, we miss them but through social media we can still talk and see each other, so it makes it better", explains Khalil, a refugee from Palestine currently living in Denmark, who uses mostly Facebook and Instagram to stay in touch with his family and friends back home.

In addition to serving as personal communication tools, social media have evolved to become important information distribution channels. This revolution in information and communication technology is of particular significance for refugees, migrants, and their families and friends, who live geographically dispersed but often remain connected through transnational networks. 


\section{NEW TO THE COUNTRY}

Refugees and migrants inevitably navigate an unfamiliar culture in their host country, especially when they first move there. They may have less friends and social networks in their new home, may need assistance and advice regarding health care, education, and employment, which in most cases operate very differently to the home country. Social media can therefore be seen as a useful tool for reaching out to newcomers, and in providing relevant information to assist in their integration. Local authorities and civil society organisations may also use platforms such as Facebook to organise outreach activities, in order to support refugees and migrants in settling down in their new homes.

'Starting again': picture taken by a Syrian refugee in Belgium.

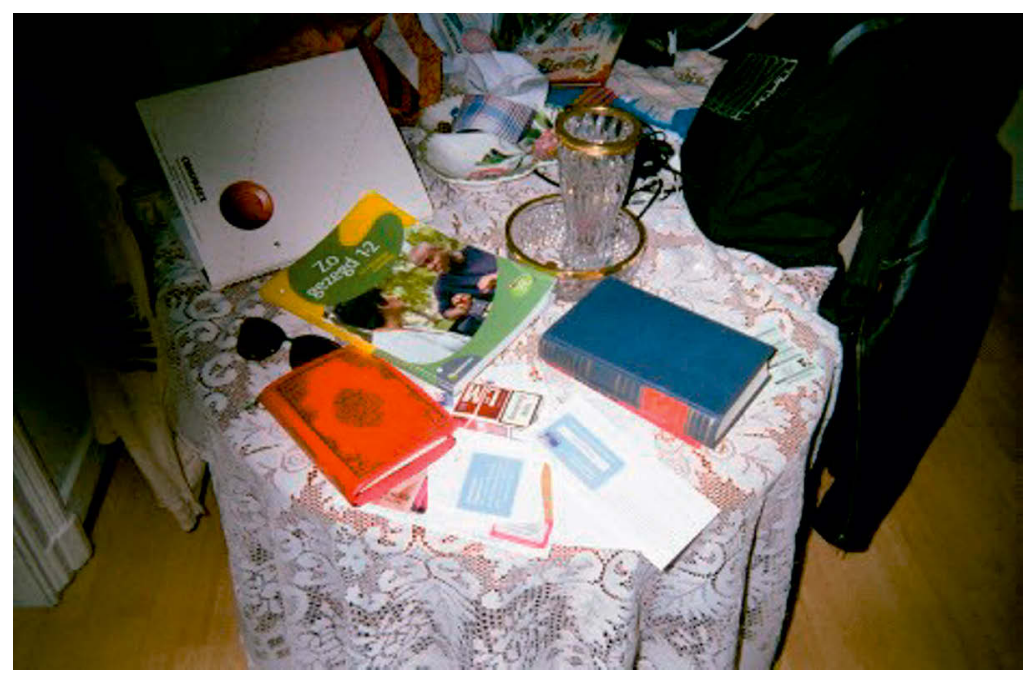

Source: refugeepostcards.com

Online platforms can even help the newcomers to become acquainted with the way of life in the new country: "Facebook has helped me to get to know more about the Danish culture as, through the Danish pages, I follow the news of the country and get to know about the things [Danes] like and don't like, and even the music they listen to", explains Khalil. 
In some cases, social media are open to everyone, thereby creating a public sphere in which information can be published. This information has the advantage that it does not originate from any authorities and that it circulates through low-key channels, spreading very rapidly. Social media thus constitute a 'backstage' space of realistic information that can be conceived of as a form of resistance to dominant structures, such as the increasingly restrictive immigration regimes of advanced societies ${ }^{1}$.

In the case of migrant and refugee networks, for example, information on upcoming rounds of legalisation, availability of informal jobs and accommodation, or illegal ways of crossing borders can spread very quickly, thus affecting the migration strategies of refugees and migrants. Social media can therefore be expected not only to strengthen people's ability to migrate, but also to feed their aspiration to migrate.

Moreover, online platforms make it possible to access a wider range of individuals to whom we are weakly tied, if at all, and are effective in developing new relationships, as they lay the groundwork for formerly unacquainted individuals to connect. Through the open structure offered by social media, users can selectively create communities based on interest rather than prior acquaintance. Consequently, latent ties - ones that exist only technically - are activated. In other words, they are converted from latent to weak ties, by some form of social interaction between users.

\section{USE OF FACEBOOK DURING THE REFUGEE MOVEMENT}

Since the start of the European refugee movement, numerous private and public social media groups have been created in the Arabic language on Facebook and other platforms, such as Whatsapp. These groups have attracted thousands of subscribers and followers ${ }^{2}$. Syrian refugees fleeing war in search of a new life in northern Europe have used social media to guide

1 Rianne Dekker \& Godfried Engbersen (2012). How social media transform migrant networks and facilitate migration. International Migration Institute, University of Oxford.

2 Tom Rayner (2015). Refugees use social media as journey guide. Sky News. 
them along their journey from Greece, through the Balkan states, to final destinations like Germany and Sweden.

The pages and groups have been used as valued sources of information for people right the way along the refugee route, to plan and organise the journey from the home country to Europe. Thousands have shared information advising those coming behind them on what to avoid, and asking questions of those in front about what to expect.

"I didn't join social media groups when planning my move to Europe, but I know that most people become members of such groups to find out which country is better to travel to and how the laws are in that country. Actually, we even have groups for refugees for each country in Europe, so you join the group and people ask each other about the laws in that country regarding refugees. You can also ask questions about life as a refugee, such as: how long does it take to get an answer for your case? How are refugees treated? Is there racism against refugees? People also ask for advice. I am a member of a Facebook group for refugees in Denmark, but I don't really use it. My sister, on the other hand, is a member of many groups", says Khalil.

\section{AN OPPORTUNITY TO REVIVE CONTACT}

Social media offer the possibility for migrants to renew contact with important weak ties, thereby acquiring bridging social capital. For migrants, information on new destinations is more likely to spread through relatively weak connections than through close contacts.

Weak ties in destination contexts may function as 'pioneers', providing information or assistance to new migrants. In some cases, these weak ties evolve to become strong ties: "I know a lot of young men who use the internet to come to Europe. There are even popular Arabic songs about this. One song is named 'Josephine'. It's about a young man who meets an older lady on the internet and starts dating her online, after a while she comes to Morocco on vacation and they eventually marry. I saw this happen when I was still living in Morocco. A friend of mine met a lady on the internet and she came to see him after 3 months of internet dating. They are still married after 10 years and even have children. So yes, many people are using the in- 
ternet but I don't know how many succeed", explains Hamza, who came from Morocco to the Netherlands in 2001 as an irregular migrant ${ }^{3}$.

Latent ties, including far-removed acquaintances or total strangers, can often initiate contact via social media to get in touch with migrants in their countries of origin, asking for information about migration to and life in the destination context.

According to Douglas, who migrated from Brazil to the Netherlands in 2003 with his family members, being contacted by strangers is not unusual: "Two guys added me on Orkut and they asked me about the Netherlands. They wanted information. [...] I also heard this once from a friend of mine. He was also added by strange people. Some people meet up here through online connections. You see that someone else also lives here so you get in touch with him and arrange a meeting. [...] It is an easy thing to do. Everyone can connect. You can see where people live and connect to the people that live in, say, the Netherlands" 4 .

\section{LENDING A HELPING HAND THROUGH SOCIAL MEDIA}

Social media pages are set up not just by those who are involved in the process of migration, but also by those in the host country, as a way to help newcomers to settle into the new and foreign society.

One such page is titled "Helping Local (Central Pa) Refugee Families in Need" which is a public Facebook group with 365 members, and was set up by two friends - Nay Rahman and Fouzia Abdellaoui - in order to help refugee families in the Central Pennsylvania area: "We started off this group as a clothing, blanket and coat drive, which had a successful turnout. After completing that portion, we wanted to keep this group active to continue to show our love and support, and to help out local refugee families who have resettled in the Central Pennsylvania area. If this interests people, we encourage them to join our group". After achieving their goal of collecting clothes and blankets for refugee families, the group has been kept active with members sharing articles and documentaries that help members to bet-

3 Dekker \& Engbersen, p. 10-11.

4 Ibid, p. 11. 
ter understand the situation of refugees, as well as posting links to charity pages that raise money for refugees.

'All I've known': picture taken in Paris by a former Somali refugee.

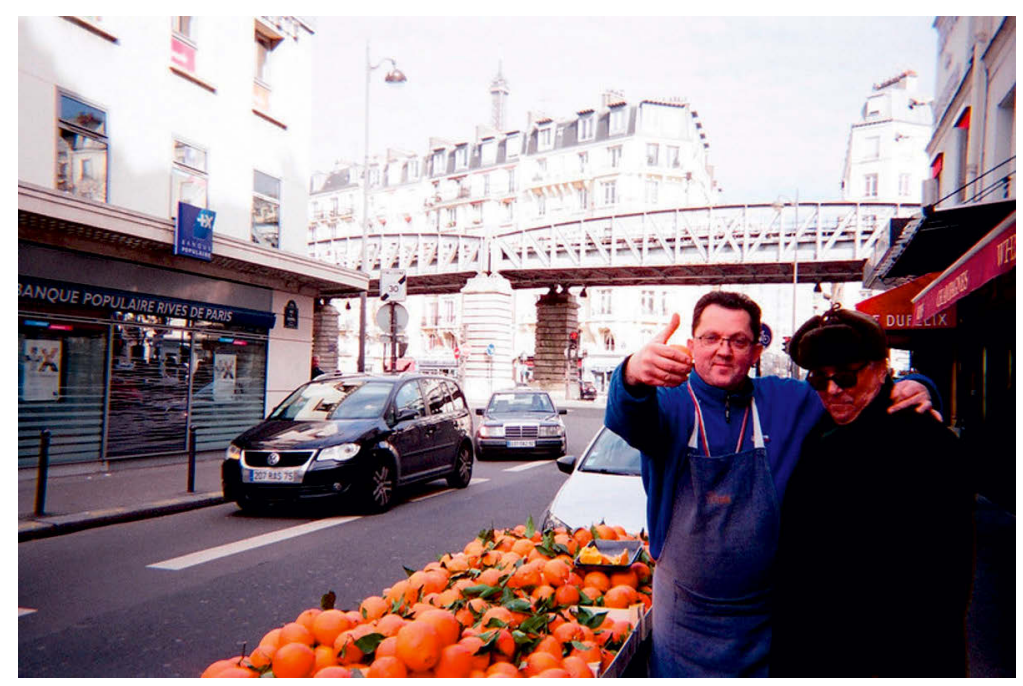

Source: refugeepostcards.com

Another public Facebook group is titled "Help the refugees in Macedonia" and has a wider reach, with 9,625 members. The group is constantly being updated with information about refugees that have been spotted in Macedonia and who might need help. A recent post read: "There [are] a group of immigrants on the island, most of them children and women in need of food assistance. 50 people. Sleeping on the street [and] do not have a place to live".

The post was accompanied by a screenshot showing the exact location of the group on Google maps, as well as pictures showing the food the refugees had been eating (beans and bread), and how they had been sleeping rough near open waters. In less than an hour of the request being posted, a member had replied with a link to the Facebook page for United Rescue Aid, suggesting the member who had initially written the post get in touch to see if the organisation could assist the group. 
An earlier message was posted on the group by a member from Euratsfeld in Austria, which shared a link to a charity page, with the following comment: "Hi friends! I want to update you about the case [of] Faisal - the disabled boy in Lebanon. Until now he just had physiotherapy, but now he needs surgery. Surgery will be on March 20th, if we can afford the costs. The surgery costs $\$ 1000$, but unfortunately now [there is] only $\$ 60$ left. I really ask for your help and support to [raise] as much as possible of the needed amount. The family cannot afford it (widow with 4 children in a camp in Lebanon) - otherwise the surgery has to be cancelled. Each little amount counts! Please help us to reach the goal and give Faisal a better future!".

This post did not have any interactions even after 17 hours of it being posted, suggesting that perhaps the members were suffering from compassion fatigue, and could provide advice but were more reluctant to assist when it came to monetary help. Perhaps this is because, as suggested by the post, members in the group had already helped to raise funds for Faisal's physiotherapy - donating more money therefore may not be feasible for everyone.

It is also interesting to note how such groups, though aimed at people from a particular country (in this case Macedonia) attract Facebook users from other countries (such as the member from Austria), who then share information about refugees in other parts of the world (such as the case of Faisal in Lebanon). This shows that social media connects not just refugees, but also people living in different parts of Europe, bringing them together to raise awareness, and to provide help, resources and assistance to those who need it. As a result, as members post in groups and share content related to refugees, latent ties are activated and converted into weak ties.

\section{MORE THAN JUST A MEANS OF COMMUNICATION}

What started off as a way to interact with friends and family, has become one of the most powerful tools of our time. When it comes to refugees and migrants, social media play a big role in their lives - before their departure from their home country, after their arrival in the host country, and beyond. They provide a way for those planning their journeys to foreign lands to 
weigh the risks, seek advice, take safety precautions, and, at times, prevent death. In the host country, online platforms are used to organise help, resources and assistance for the newcomers, in the form of clothes, food, advice and donations - such help is crucial for the integration of migrants once they're in the new society.

Whilst face-to-face interaction is also often necessary, social media acts as a safety net for those who have just moved thousands of miles away from home, enabling migrants and refugees to seek help despite potential communication barriers, and at the same time communicate with family and friends back home during difficult times.

But according to Luiz, who came from Brazil to the Netherlands in 2001 to marry a Dutch partner, how the experience of migration is portrayed by migrants on social media is misleading: "What I see a lot is that Brazilians put all their photos on Facebook or Orkut. So there is this image that life in the Netherlands is very pleasurable and nice. It spreads a wrong idea of what [is actually the case] here. There are people that get totally misled" 5 .

Social media may facilitate the journeys of migrants and refugees, but nothing can completely prepare them for what is to come once they arrive at their new homes.

5 Dekker \& Engbersen, p. 15. 



\title{
Waiting Room. A Short Essay Film
}

\author{
Tuna Kaptan / Mate Ugrin
}

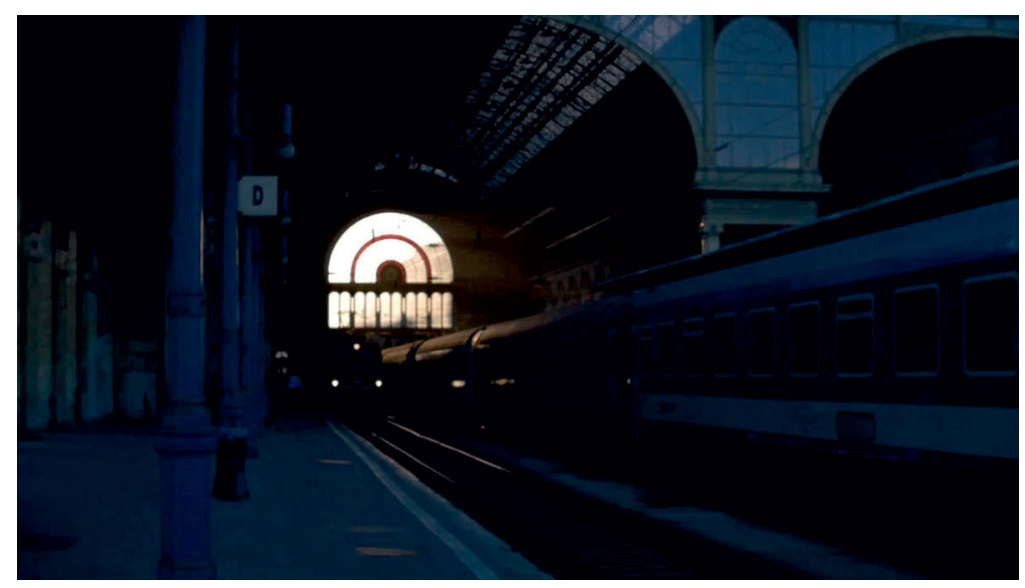

Waiting room is a short essay film dealing with the recent European migrant crisis. The inadequate reactions from Europe's leading countries had irreversible repercussions upon the lives of many refugees. The aim of the film is to depict the condition of transit by reconstructing an experience of a Syrian immigrant unable to continue his journey.

The protagonist of the film, a thirty-three year old photo journalist from Damascus, confronts us with a fragmented story from the Keleti station in Budapest, while on his way to Germany. His narration is combined with recent clips from the station which are characterised by rich colours and suggestive chiaroscuro, trying to depict the distorted perception of time of the 
protagonist. By using long static shots of people passing by in slow-motion the film comments travel as a form of privilege.
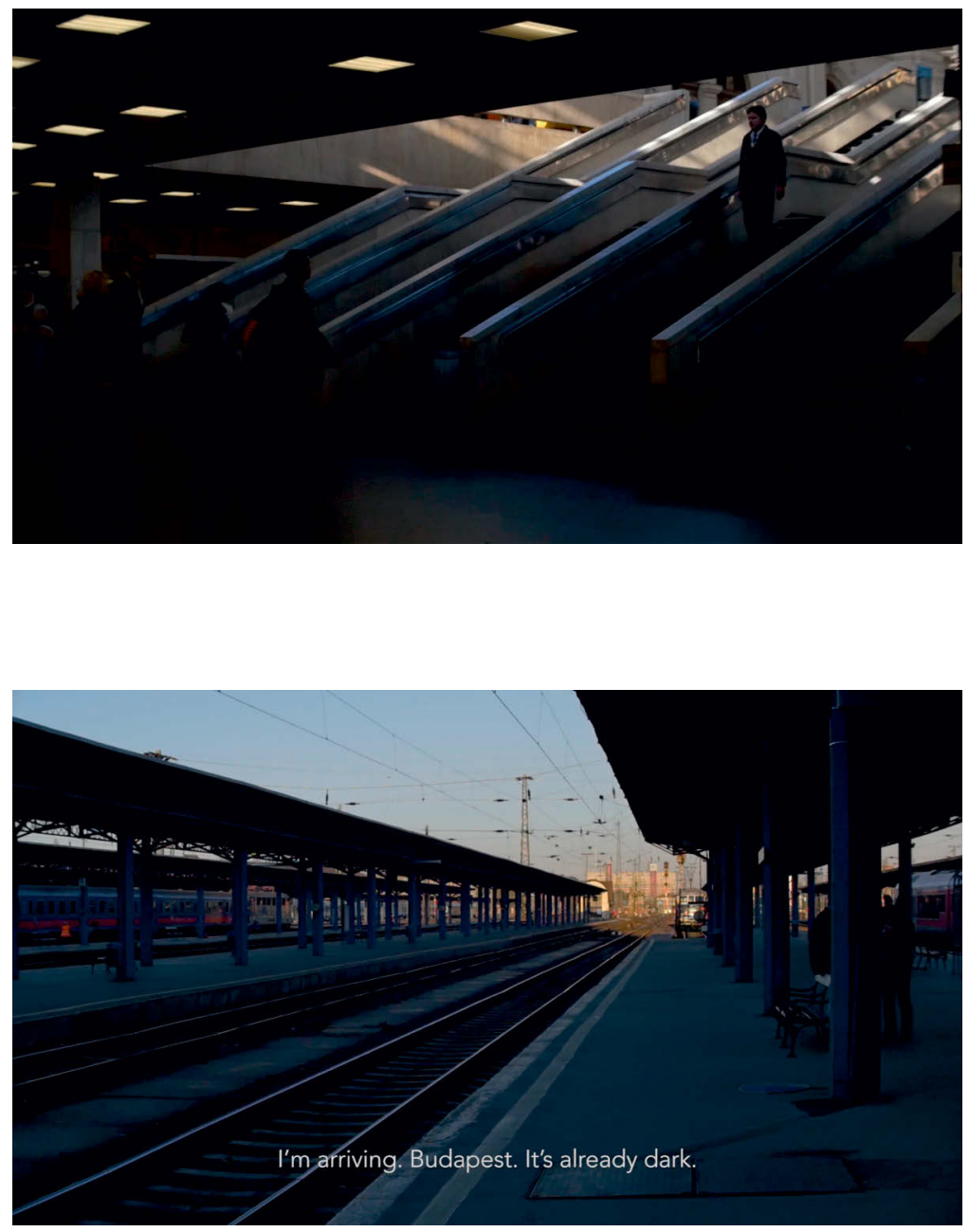
While as a tourist we mostly feel welcome and appreciated, the situation with migrants is radically different. During the most recent migration crisis the Keleti station becomes the real and symbolical place of collapsing of values like humanism and solidarity which are replaced by outbursts of violence and xenophobia. The shots of the station do not expose us to the moments when Keleti was resembling an immigration camp, which gives us space to reflect upon something that was considered unimaginable to happen in Europe. The protagonist evokes his own traumatic and tiring migration experience of being in transit in a hostile environment, the tedious feeling of anxiety is almost palpable.

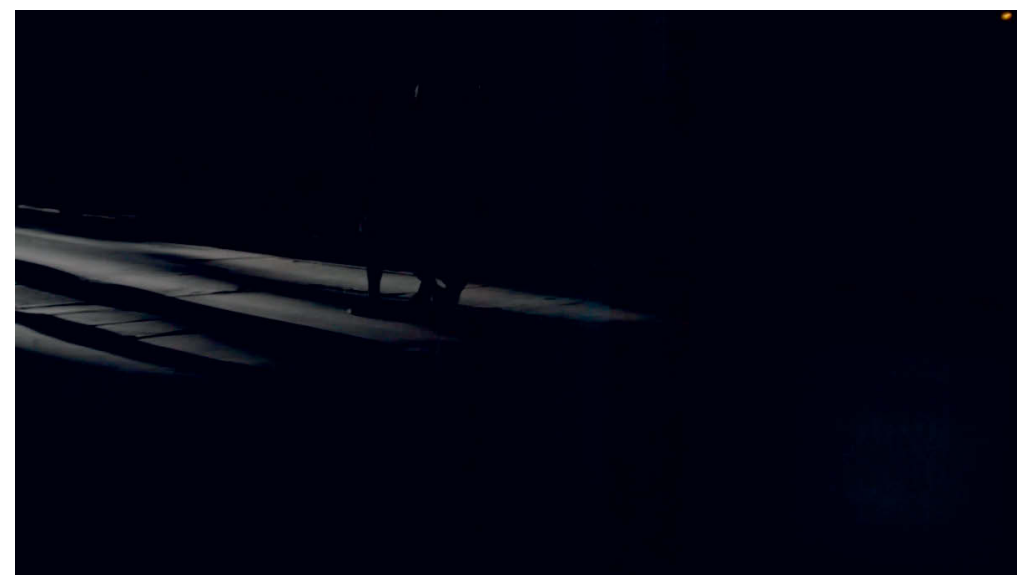

Link to the film:

https://vimeo.com/260457280

password: waiting 



\section{Autorinnen und Autoren}

Abbasi, Sara hat ihren Master in Journalismus, Medien und Globalisierung an der Universität Hamburg abgeschlossen. In ihrer Masterarbeit hat sie die Onlinemedien-Nutzung von verfolgten Minderheiten untersucht. Im Moment arbeitet Sara in London im Bereich Corporate Governance und macht als Freelancerin Jounalismus-Projekte. Sara hat das „Refugee.Postcards" Projekt gegründet, das von der Alfred-Töpfer-Stiftung unterstützt wird.

Hochleitner, Anna hat in Passau Kulturwirtschaft und Business Administration and Economics studiert und im Anschluss daran einen Master in Economics und Development Economics an den Universitäten Nottingham und Konstanz absolviert. Von 2014 bis 2017 war sie Stipendiatin der Studienstiftung und des Max Weber Programms. Derzeit promoviert sie in Nottingham und beschäftigt sich dabei mit entwicklungs- und verhaltensökonomischen Fragestellungen.

Imgarten, Nils studierte von 2013 bis 2019 Rechtswissenschaften an den Universitäten Göttingen und Galway (Irland). Er war 2015 Mitbegründer der Refugee Law Clinic Göttingen. Seine Interessenschwerpunkte liegen im Migrations- und Europarecht.

Inagaki, Kentaro hat Germanistik und Geschichte an der Universität Tokyo studiert. Er schreibt zur Zeit an einer Dissertation zum Thema Politische Theorie und zur Auslegung der Bibel in der Frühen Neuzeit.

Kania, Merve M.Sc., ist eine Masterstudentin in War Studies am King's College London. Sie hat einen M.Sc. in Middle East Politics von der 
School of Oriental and African Studies (SOAS), University of London (mit Auszeichnung) und einen B.A. in Internationale Beziehungen und Staatswissenschaften-Sozialwissenschaften von der Universität Erfurt. Ihre Forschungsinteressen umfassen die Politiken der Sicherheit, Konflikte, Identitäten und des Wiederaufbaus, insbesondere bezüglich Syrien, Libanon, der Türkei sowie Migration und Flüchtlingen.

Kaptan, Tuna, Studium der Theater- und Medienwissenschaft und der Filmregie an der HFF München und der ENERC Buenos Aires. Seine Filme liefen zahlreich auf internationalen Filmfestivals und wurden mehrfach ausgezeichnet, u.a. "Nacht Grenze Morgen" auf der IDFA und "Schildkröten Panzer" auf DOK Leipzig.

Känner, Lise hat von 2013 bis 2018 an der Heinrich-Heine-Universität Düsseldorf Rechtswissenschaft studiert und erhielt dort unter anderem für ihre besonderen Leistungen im Schwerpunktbereich „Recht der Politik“ den „Brückenschlag“-Preis der Moe Radzyner Stiftung. Ihre Forschungsschwerpunkte liegen im europäischen und nationalen Asyl- und Migrationsrecht sowie im Verfassungsrecht. Seit 2016 engagiert sie sich bei der Refugee Law Clinic Düsseldorf, einem gemeinnützigen Verein, der kostenlose Rechtsberatung für Geflüchtete anbietet.

Kirsch, Thea studierte von 2012 bis 2016 Politik- und Kommunikationswissenschaft mit dem Schwerpunkt kritische Migrations- und Sicherheitsstudien an der Technischen Universität Dresden und der Boston University (USA). Seit 2016 ist sie Masterstudentin der Politikwissenschaft an der Freien Universität Berlin. In ihrer Forschung untersucht Thea Kirsch digitale Systeme sozialer und politischer Kontrolle durch Rangbildung, Hierarchisierung und Klassifikation. Ihr besonderes Interesse gilt der Erhebung, Speicherung und Nutzung von biometrischen Daten.

Pichl, Maximilian forscht im Rahmen eines Projektes der Hans-BöcklerStiftung an der Universität Kassel zur Krise der EU-Migrationspolitik. Er studierte Rechts- und Politikwissenschaft in Frankfurt am Main. Zwischen 2015 bis 2017 war er rechtspolitischer Referent von PRO ASYL e.V. und ist im Vorstand vom Bundesfachverband unbegleitete minderjährige Flüchtlinge (BUMF e.V.). 
Tohidipur, Timo, Dr. jur., ist Professor am Fachbereich Soziale Arbeit und Gesundheit der Frankfurt University of Applied Sciences, Frankfurt am Main. Seine Forschungsschwerpunkte sind das Europäische Grenzregime, Migrationsrecht, Rechtsvergleichung und Recht im Film.

Ugrin, Mate, 2012 Abschluss Filmstudium an der Academy of Arts in Belgrad, Serbien. Anschließend Master of Fine Arts an der Hochschule für bildende Künste Hamburg und Meisterschüler-Programm an der Universität der Künste Berlin. Für seinen Abschlussfilm "In der Zwischenzeit / Meanwhile" gewann er 2018 den FIRST STEPS Award in der Kategorie "Kurzund Animationsfilm". 


\section{Politikwissenschaft}

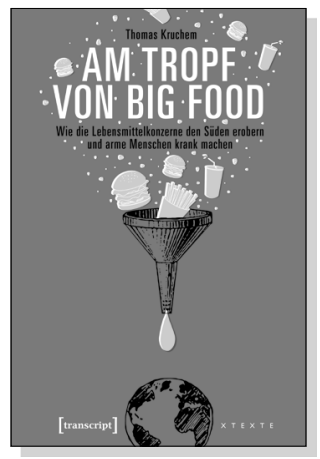

Thomas Kruchem

Am Tropf von Big Food

Wie die Lebensmittelkonzerne den Süden erobern

und arme Menschen krank machen

2017, 214 S., kart., zahlr. Abb.

$19,99 €(D E), 978-3-8376-3965-0$

E-Book: 16,99 € (DE), ISBN 978-3-8394-3965-4

EPUB: $16,99 €(D E)$, ISBN 978-3-7328-3965-0

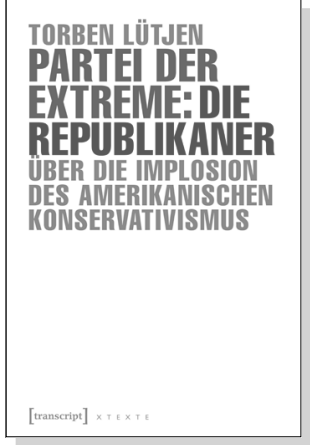

Torben Lütjen

Partei der Extreme: Die Republikaner

Über die Implosion des amerikanischen Konservativismus

2016, 148 S., kart.

$14,99 €(D E), 978-3-8376-3609-3$

E-Book: 12,99 € (DE), ISBN 978-3-8394-3609-7

EPUB: $12,99 €$ (DE), ISBN 978-3-7328-3609-3

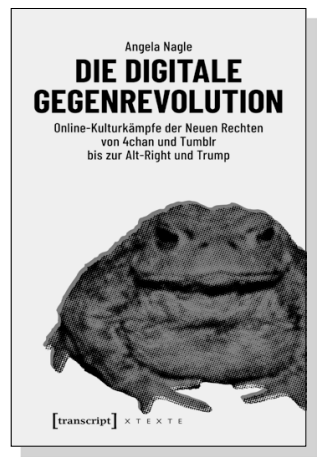

\section{Angela Nagle}

\section{Die digitale Gegenrevolution}

Online-Kulturkämpfe der Neuen Rechten von 4chan und Tumblr bis zur Alt-Right und Trump

2018,148 S., kart.

$19,99 €(D E), 978-3-8376-4397-8$

E-Book: 17,99 € (DE), ISBN 978-3-8394-4397-2

EPUB: $17,99 €$ (DE), ISBN 978-3-7328-4397-8 


\section{Politikwissenschaft}

Ines-Jacqueline Werkner Gerechter Frieden Das fortwährende Dilemma militärischer Gewalt

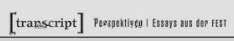
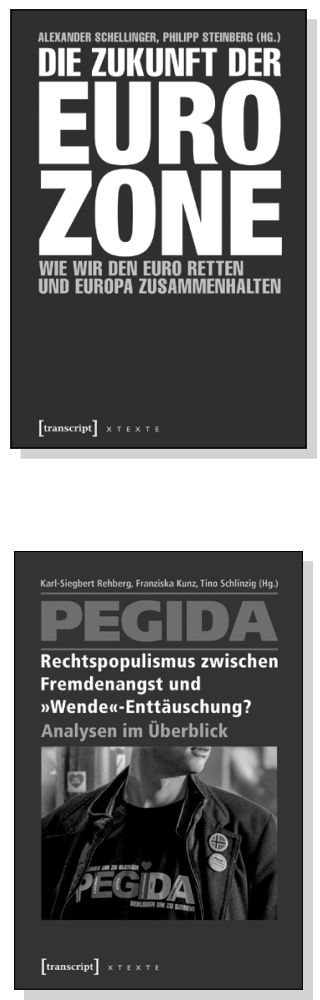

Ines-Jacqueline Werkner

\section{Gerechter Frieden}

Das fortwährende Dilemma militärischer Gewalt

2018,106 S., kart.

$14,99 €(D E), 978-3-8376-4074-8$

E-Book: 12,99 € (DE), ISBN 978-3-8394-4074-2

Alexander Schellinger, Philipp Steinberg (Hg.)

\section{Die Zukunft der Eurozone}

Wie wir den Euro retten und Europa zusammenhalten

2016, 222 S., kart.

$19,99 €(D E), 978-3-8376-3636-9$

E-Book: 17,99 € (DE), ISBN 978-3-8394-3636-3

EPUB: 17,99 € (DE), ISBN 978-3-7328-3636-9

Karl-Siegbert Rehberg, Franziska Kunz, Tino Schlinzig (Hg.)

\section{PEGIDA -}

\section{Rechtspopulismus zwischen Fremdenangst} und "Wende «-Enttäuschung?

Analysen im Überblick

2016, 384 S., kart.

29,99€ (DE), 978-3-8376-3658-1

E-Book: $26,99 €$ (DE), ISBN 978-3-8394-3658-5

EPUB: $26,99 €$ (DE), ISBN 978-3-7328-3658-1 
